\title{
Travelling Waves for Adaptive Grid Discretizations of Reaction Diffusion Systems I: Well-Posedness
}

\author{
H. J. Hupkes ${ }^{1}$ (D) E. S. Van Vleck ${ }^{2}$
}

Received: 23 October 2019 / Revised: 29 October 2020 / Accepted: 21 May 2021 /

Published online: 14 July 2021

(c) The Author(s) 2021

\begin{abstract}
In this paper we consider a spatial discretization scheme with an adaptive grid for the Nagumo PDE. In particular, we consider a commonly used time dependent moving mesh method that aims to equidistribute the arclength of the solution under consideration. We assume that the discrete analogue of this equidistribution is strictly enforced, which allows us to reduce the effective dynamics to a scalar non-local problem with infinite range interactions. We show that this reduced problem is well-posed and obtain useful estimates on the resulting nonlinearities. In the sequel papers (Hupkes and Van Vleck in Travelling waves for adaptive grid discretizations of reaction diffusion systems II: linear theory; Travelling waves for adaptive grid discretizations of reaction diffusion systems III: nonlinear theory) we use these estimates to show that travelling waves persist under these adaptive spatial discretizations.
\end{abstract}

Keywords Travelling waves $\cdot$ Adaptive grids $\cdot$ Singular perturbations $\cdot$ Spatial discretizations

Mathematics Subject Classification $34 \mathrm{~K} 31 \cdot 37 \mathrm{~L} 15$

\section{Introduction}

In this paper we consider adaptive discretization schemes for a class of scalar reactiondiffusion equations that includes the Nagumo PDE

$$
u_{t}=u_{x x}+g_{\mathrm{cub}}(u ; a),
$$

with the bistable cubic nonlinearity

$$
g_{\text {cub }}(u)=u(1-u)(u-a), \quad 0<a<1 .
$$

$\bowtie$ H. J. Hupkes

hhupkes@math.leidenuniv.nl

E. S. Van Vleck

erikvv@ku.edu

1 Mathematisch Instituut, Universiteit Leiden, P.O. Box 9512, 2300 RA Leiden, The Netherlands

2 Department of Mathematics, University of Kansas, 1460, Jayhawk Blvd, Lawrence, KS 66045, USA 
In particular, we discretize (1.1) on a time-dependent spatial grid and add an extra equation that aims to distribute the gridpoints in such a way that the arclength of the solution is equal between any two consecutive gridpoints.

Our main contribution in this series of papers is to show that the resulting coupled semidiscrete system is well-posed and admits solutions that can be interpreted as travelling waves. In particular, our results here are part of an ongoing program that aims to systematically explore the impact of commonly used spatial, temporal and full discretization schemes on the dynamical behaviour of dissipative PDEs.

\subsection{Reaction-Diffusion Systems}

Reaction-diffusion PDEs have been extensively studied in the past decades. Indeed, their rich pattern-forming properties allow many intriguing localized structures that can be observed in nature to be reproduced analytically and numerically. For example, the classical paper by Aronson and Weinberger [1] shows how (1.1) and its higher-dimensional counterparts can be used to model the spreading of a dominant biological species throughout a spatial domain. Upon adding a slowly-varying second component to (1.1) by writing

$$
\begin{aligned}
u_{t} & =u_{x x}+g_{\mathrm{cub}}(u ; a)-v, \\
v_{t} & =\epsilon(u-v),
\end{aligned}
$$

Fitzhugh [22,23] was able to effectively describe the propagation of signal spikes through nerve fibres. Sparked by his interest in morphogenesis, Turing [48] described the famous bifurcation through which equilibria of general two-component reaction-diffusion systems can destabilize and generate spatially periodic structures such as spots and stripes.

These early results led to the development of many important technical tools that today are indispensable to the field of dynamical systems. For example, comparison principle techniques have been used to study the global dynamics of (1.1) in one [21] and two [6] spatial dimensions. The rigorous construction of the pulses observed by FitzHugh for (1.3) led to the birth of geometric singular perturbation theory [11,24,37]. The development of Evans function [34] and semigroup theory [45] was heavily influenced by the desire to analyze the stability of many of these localized structures.

The systems (1.1) and (1.3) are both still under active investigation. For example, the behaviour of perturbed spherical [44] or planar [39] fronts has been investigated for higherdimensional versions of (1.1). In addition, in [12,13] the authors consider (1.3) in the $a \downarrow 0$ limit and describe the birth of pulse solutions with oscillating tails.

\subsection{Discretized Travelling Waves}

It is well-known that travelling waves play an important role in the global dynamics of (1.1). Indeed, they have a large basin of attraction and can be used as building blocks to construct more complicated solutions [21,51,52]. As a consequence, a line of research has developed in recent years to investigate in what sense these travelling waves survive common discretization schemes.

As a first step, it is natural to introduce the approximants $U_{j}(t) \sim u(j h, t)$ and apply a standard discretization to the second derivative in (1.1). In this fashion one obtains the lattice differential equation (LDE) 


$$
\dot{U}_{j}(t)=\frac{1}{h^{2}}\left[U_{j-1}(t)+U_{j+1}(t)-2 U_{j}(t)\right]+g_{\text {cub }}\left(U_{j} ; a\right),
$$

which can be seen as the nearest-neighbour spatial discretization of the PDE (1.1) on the grid $h \mathbb{Z}$. Of course, the $0<h \ll 1$ regime is the most interesting from the perspective of numerical analysis.

However, we remark here that many physical and biological systems have a discrete spatial structure for which it is natural to take $h \sim 1$. Indeed, genuinely discrete phenomena such as phase transitions in Ising models [3], crystal growth in materials [10], propagation of action potentials in myelinated nerve fibers [5] and phase mixing in martensitic structures [49] have all been modelled using equations similar to (1.4). As a consequence, the system (1.4) has attracted a great deal of attraction, see e.g. $[7,14,35,38,53]$ or the survey paper [30] for a more detailed overview.

As a second step, one can consider full spatial-temporal discretizations of (1.1) by replacing the remaining temporal derivative in (1.4). For instance, one can apply the backward-Euler discretization scheme with time-step $\Delta t$ to arrive at the system

$$
\begin{aligned}
\frac{1}{\Delta t}\left[U_{j}(n \Delta t)-U_{j}((n-1) \Delta t)\right]= & \frac{1}{h^{2}}\left[U_{j-1}(n \Delta t)+U_{j+1}(n \Delta t)-2 U_{j}(n \Delta t)\right] \\
& +g_{\text {cub }}\left(U_{j}(n \Delta t) ; a\right) .
\end{aligned}
$$

This type of system is commonly referred to as a coupled map lattice (CML). Such systems are used as stand-alone models across a wide range of disciplines, from the construction of hash functions [50] to the study of population dynamics [17]. Several authors have considered travelling wave solutions to this type of CML [15,18-20]; see [30] or the sequel papers [31,32] for a more detailed overview.

\subsection{Arclength Equidistribution}

Both of the discretization schemes introduced above involve gridpoints that remain stationary. However, most efficient modern solvers do not use fixed spatial grids but concentrate their meshpoints in areas where the solution under construction fluctuates the most. In particular, let us write $\left\{x_{j}(t)\right\}$ for the positions of the grid points. Introducing the approximants

$$
U_{j}(t) \approx u\left(x_{j}(t), t\right),
$$

we may use (1.1) to compute

$$
\begin{aligned}
\frac{d}{d t} U_{j}(t) & =u_{x}\left(x_{j}(t), t\right) \dot{x}_{j}(t)+u_{t}\left(x_{j}(t), t\right) \\
& =u_{x}\left(x_{j}(t), t\right) \dot{x}_{j}(t)+u_{x x}\left(x_{j}(t), t\right)+g_{\operatorname{cub}}\left(u\left(x_{j}(t), t\right) ; a\right)
\end{aligned}
$$

in the special case that the approximation (1.6) is exact. Using central differences to discretize the spatial derivatives in (1.7) on the grid $x_{j}(t)$, we obtain the LDE

$$
\dot{U}_{j}=\left[\frac{U_{j+1}-U_{j-1}}{x_{j+1}-x_{j-1}}\right] \dot{x}_{j}+\frac{2}{x_{j+1}-x_{j-1}}\left[\frac{U_{j-1}-U_{j}}{x_{j}-x_{j-1}}+\frac{U_{j+1}-U_{j}}{x_{j+1}-x_{j}}\right]+g_{\mathrm{cub}}\left(U_{j} ; a\right) .
$$

This system should be compared to [29, Eqs. (1.12)-(1.13)] where a similar procedure was applied to Burgers' equation. 
In order to close the system, we need to describe the behaviour of the gridpoints. For illustrative purposes, let us consider the so-called MMPDE5 method that was originally developed by Huang, Ren, and Russell $[27,28,43]$. This method is efficient and relatively easy to formulate for our problem. In particular, inspecting [29, Eqs. (2.52), (2.53), (2.57)], the gridpoint behaviour can be described by

$$
\sigma \dot{x}_{j}=\sqrt{\left(x_{j+1}-x_{j}\right)^{2}+\left(U_{j+1}-U_{j}\right)^{2}}-\sqrt{\left(x_{j-1}-x_{j}\right)^{2}+\left(U_{j-1}-U_{j}\right)^{2}},
$$

in which $\sigma>0$ is a tunable speed parameter. In the terminology of [29], we are using the arclength monitor function

$$
\rho(x, t)=\sqrt{1+u_{x}^{2}}
$$

Indeed, the update rule (1.9) acts to equalize the arclength of the solution profile between grid-points.

\subsection{Adaptive Meshing}

Numerical techniques involving non-constant grids have attracted tremendous attention in the search for accurate and efficient approximation procedures for differential equations. The first method of this type that is based upon an equidistribution principle was described by de Boor [16]. The method was developed to efficiently solve boundary value problems for ordinary differential equations. After each step in the numerical iteration scheme, the error is computed in a pointwise fashion. One can subsequently choose new gridpoints in such a way that this error is equally distributed over each subinterval in the new mesh. This technique turned out to be very effective and has also been used for time dependent (parabolic) PDEs in one space dimension.

The MMPDE5 method described above is an r-adaptive refinement scheme in the terminology of the finite element community, since the mesh is continuously relocating as it adapts to the solution of the PDE being solved. The equations that determine the movement of the mesh are generally independent of the PDE being solved, but are dependent on the solution of the underlying physical PDE. Several approaches have been developed that are relatively simple to program and robust with respect to the choice of adjustable parameters. The recent book [29] contains a comprehensive treatment of the most important moving mesh methods, including the MMPDE5 scheme described above. Further references can be found in the review articles [9] and [25]. The literature concerning convergence results for moving mesh methods is somewhat limited. Results have been obtained $[4,41,42]$ for finite difference methods applied to singularly perturbed two-point boundary value problems and reaction-diffusion equations. However, these require a-priori knowledge of the mesh behaviour and explicitly use the singular part of the exact solution. Results that do not require such a-priori knowledge are available for linear one-dimensional elliptic equations [2] and one-dimensional quasi-linear convection-diffusion problems [36]. For combustion PDEs that feature blow-up behaviour, one can use scaling invariance and moving mesh methods to recreate the scaling laws inherent in the exact blow-up solutions [8]. Finally, the behavior of moving mesh schemes for travelling wave solutions of the Fisher equation, which is the monostable counterpart of (1.1), was investigated in [40]. 


\subsection{Program Overview}

Inspection of the coupled system (1.8)-(1.9) shows that one loses the comparison principle, even if $x$ is treated as a known function. Such drastic structural changes are a common feature when applying discretization schemes and we refer to [47] for an interesting discussion. For our purposes here, this means that we will have to consider perturbative techniques to analyze (1.8)-(1.9), viewing the speed parameter $\sigma$ and the average arclength between gridpoints as small parameters.

In this series of papers we focus on the singular case $\sigma=0$, which allows us to rewrite (1.9) as

$$
h=\sqrt{\left(x_{j+1}-x_{j}\right)^{2}+\left(U_{j+1}-U_{j}\right)^{2}}=\sqrt{\left(x_{j-1}-x_{j}\right)^{2}+\left(U_{j-1}-U_{j}\right)^{2}}
$$

for some constant $h>0$ that we take to be small. In particular, we obtain

$$
x_{j+1}-x_{j}=\sqrt{h^{2}-\left(U_{j+1}-U_{j}\right)^{2}}
$$

for all $j \in \mathbb{Z}$. In order to fix the absolute positions of the gridpoints, we impose the boundary condition

$$
\lim _{j \rightarrow-\infty} x_{j}(t)-j h=0
$$

at each time $t \geq 0$.

Our main results in this paper show how the variable $x$ can be eliminated from (1.8) and establish the well-posedness of the resulting equation for $U$. We continue in [31] by studying the linearization of this equation around the (appropriately transformed) PDE waves $u(x, t)=\Phi_{*}\left(x+c_{*} t\right)$ that satisfy (1.1). In particular, we transfer Fredholm properties from the continuum to the discrete regime. These properties are subsequently used in [32] to construct travelling wave solutions

$$
U_{j}(t)=\Phi\left(x_{j}(t)+c t\right)
$$

that satisfy the boundary conditions $\Phi(-\infty)=0$ and $\Phi(+\infty)=1$. These travelling waves $(\Phi, c)$ are small perturbations of the PDE waves $\left(\Phi_{*}, c_{*}\right)$ mentioned above.

We believe that it should be possible to extend the approach developed in this series to handle the case $\sigma>0$ and to consider multi-component and/or fully-discretized systems. Recently several tools have been developed that could help to handle these generalizations $[33,46]$ and we refer to $[32, \S 1]$ for a full discussion.

\subsection{Continuum Limit}

In order to set the stage, we first briefly discuss the case where the gridpoints $x$ are not indexed by the discrete variable $j$ but by a continuous arclength parameter $\theta$. In particular, we write $x=x(\theta, t)$ and assume that $\theta=\theta(x, t)$ satisfies the arclength constraint

$$
\theta_{x}=\sqrt{1+u_{x}^{2}}
$$

We now introduce the new variables

$$
w(\theta, t)=u(x(\theta, t), t), \quad \gamma(\theta, t)=\sqrt{1-w_{\theta}(\theta, t)^{2}},
$$


which in view of the identity $u_{x}^{2}=w_{\theta}^{2} \theta_{x}^{2}$ allows us to compute

$$
\theta_{x}(x, t)=\gamma^{-1}(\theta(x, t), t), \quad x_{\theta}(\theta, t)=\gamma(\theta, t) .
$$

In particular, we find

$$
u_{x}=\gamma^{-1} w_{\theta}, \quad u_{x x}=\gamma^{-4} w_{\theta \theta},
$$

which allows us to recast the governing equation $w_{t}=u_{x} x_{t}+u_{x x}+g_{\mathrm{cub}}(u)$ as

$$
w_{t}=\gamma^{-1} w_{\theta} x_{t}+\gamma^{-4} w_{\theta \theta}+g_{\mathrm{cub}}(w) .
$$

Setting out to eliminate the $x_{t}$ term, we differentiate the second expression in (1.17) to find $\dot{x}_{\theta}=-\gamma^{-1} w_{\theta} w_{t \theta}$. Substituting this into (1.19) yields the system

$$
-\gamma^{-1} w_{\theta} \partial_{\theta}\left[\gamma^{-4} w_{\theta \theta}+g_{\text {cub }}(w)\right]=x_{t \theta}+\gamma^{-1} w_{\theta} \partial_{\theta}\left[\gamma^{-1} w_{\theta} x_{t}\right]=\gamma^{-1} \partial_{\theta}\left[\gamma^{-1} x_{t}\right] \text {. }
$$

Upon introducing the notation $\left[\int_{-} f\right](\theta)=\int_{-\infty}^{\theta} f\left(\theta^{\prime}\right) d \theta^{\prime}$ and integrating by parts, this can be solved to yield

$$
\begin{aligned}
x_{t} & =-\gamma \int_{-} w_{\theta} \partial_{\theta}\left[\gamma^{-4} w_{\theta \theta}+g_{\text {cub }}(w)\right] \\
& =-\gamma w_{\theta}\left(\gamma^{-4} w_{\theta \theta}+g_{\text {cub }}(w)\right)+\gamma \int_{-}\left(\gamma^{-4} w_{\theta \theta}+g_{\text {cub }}(w)\right) w_{\theta \theta} .
\end{aligned}
$$

Substituting this back into (1.19), we arrive at our final reference system

$$
w_{t}=\gamma^{-2} w_{\theta \theta}+\gamma^{2} g_{\mathrm{cub}}(w)+w_{\theta} \int_{-}\left(\gamma^{-4} w_{\theta \theta}+g_{\mathrm{cub}}(w)\right) w_{\theta \theta} .
$$

Unlike the original semi-linear PDE (1.1), this transformed PDE is fully nonlinear.

\subsection{Reduction Procedure}

Our main task in the present paper is to generalize the reduction procedure above to the discrete setting where $h>0$. In particular, we need to eliminate the variable $x$ from (1.8). A major complication is that the convenient integrating factor in (1.20) can now no longer be easily identified and explicitly evaluated.

In view of the boundary condition (1.13), we can repeatedly apply (1.12) to obtain

$$
\begin{aligned}
x_{k}-k h & =\sum_{j=-\infty}^{k-1}\left(\sqrt{h^{2}-\left(U_{j+1}-U_{j}\right)^{2}}-h\right) \\
& =-\sum_{j=-\infty}^{k-1} \frac{\left(U_{j+1}-U_{j}\right)^{2}}{\sqrt{h^{2}-\left(U_{j+1}-U_{j}\right)^{2}}+h} .
\end{aligned}
$$

Upon introducing the discrete derivative

$$
\left[\partial_{h}^{+} U\right]_{j}=h^{-1}\left[U_{j+1}-U_{j}\right],
$$

we note that the two conditions

$$
\left\|\partial_{h}^{+} U\right\|_{\infty}<1, \quad \partial_{h}^{+} U \in \ell^{2}
$$

are sufficient to ensure that (1.23) is well-defined. 
A direct differentiation yields

$$
\dot{x}_{k}=-\sum_{j=-\infty}^{k-1} \frac{U_{j+1}-U_{j}}{\sqrt{h^{2}-\left(U_{j+1}-U_{j}\right)^{2}}}\left(\dot{U}_{j+1}-\dot{U}_{j}\right),
$$

which is well-defined if also $\partial_{h}^{+} \dot{U} \in \ell^{2}$. Using (1.8) to eliminate $\partial_{h}^{+} \dot{U}$, we obtain the implicit expression

$$
\dot{x}_{k}=\sum_{j=-\infty}^{k-1} \mathcal{F}\left(U_{j-1}, U_{j+1}, U_{j}, U_{j+2}, \dot{x}_{j+1}, \dot{x}_{j}\right) .
$$

We show in $\S 4$ that this equation has a unique solution that can be written as

$$
\dot{x}_{k}=\mathcal{Y}_{k}\left(\left\{U_{j}\right\}_{j \leq k},\left\{\partial_{h}^{+} U_{j}\right\}_{j \leq k},\left\{\partial_{h}^{+} \partial_{h}^{+} U_{j}\right\}_{j \leq k-1},\left\{\partial_{h}^{+} \partial_{h}^{+} \partial_{h}^{+} U_{j}\right\}_{j \leq k-2}\right)
$$

for some (convoluted) function $\mathcal{Y}$. Using (1.12) to eliminate the remaining terms involving $x$ from (1.8), this allows us to write

$$
\dot{U}_{k}=\mathcal{G}_{k}\left(\left\{U_{j}\right\}_{j \leq k},\left\{\partial_{h}^{+} U_{j}\right\}_{j \leq k},\left\{\partial_{h}^{+} \partial_{h}^{+} U_{j}\right\}_{j \leq k-1},\left\{\partial_{h}^{+} \partial_{h}^{+} \partial_{h}^{+} U_{j}\right\}_{j \leq k-2}\right)
$$

for some function $\mathcal{G}$ that we describe explicitly in $\S 6$. We note that the partial derivatives of $\mathcal{G}$ can be controlled uniformly for small $h$, so the representation (1.29) isolates all the terms that have the potential to blow up as $h \downarrow 0$. By choosing an appropriate space for the sequences $U$, we show in $\S 8$ that (1.29) can be seen as a well-posed initial value problem.

The discrete third derivative in (1.29) arises directly from (1.26), which forces us to take a discrete derivative of our second-order original system. Fortunately, as in (1.21), one can use a discrete summation-by-parts technique to eliminate this derivative. The price that needs to be paid is that the right-hand-side of (1.29) becomes rather convoluted, containing terms of the form $\left(\partial_{h}^{+} \partial_{h}^{+} U\right)^{2}$. Using PDE terminology, the equation again becomes fully nonlinear rather than semi-linear and this requires considerable care.

We are aided by the special structure of $\mathcal{G}$, which is a product of two sums. More precisely, taking a discrete derivative of $\mathcal{G}$ does not involve fourth-order discrete derivatives of $U$. In fact, taking a discrete derivative of (1.29) leads to a quasi-linear third-order equation that plays a major role in our construction.

As can be expected, the expressions that arise from this whole procedure are quite unwieldy. In fact, one of the most important tasks in this series of papers is to develop a framework that allows us to extract and simplify the components that are essential for our analysis, while keeping the resulting errors under control. As a consequence, a significant portion of this paper is devoted to bookkeeping issues. At times, this leads to results that appear to have been written by accountants rather than mathematicians, masking an elementary estimate behind a veil of notation. This is the price that needs to be paid to enable the subsequent analysis in [31,32]. In addition, we are confident that our techniques can be used as a template for further studies in this area.

\subsection{Overview}

This paper is organized as follows. Our main results are formulated in $\S 2$. We introduce some useful terminology and sequence spaces in $\$ 3$, together with some basic tools that link discrete and continuous calculus. We continue in $\S 4$ by discussing the behaviour of the 
gridpoints. In particular, we derive an equation for the nonlinearity $\mathcal{Y}$ that describes $\dot{x}$. After a technical intermezzo in $\S 5$, we use the expression for $\mathcal{Y}$ in $\S 6$ to analyze the function $\mathcal{G}$ that appears in the reduced scalar LDE (1.29). More specifically, we perform an initial summation by parts procedure to eliminate the third discrete derivative. In $\S 7$ we formulate estimates on all the nonlinear functions that appear as factors in the product structure of $\mathcal{G}$. These estimates are used in $\S 8$ to establish our well-posedness results. In addition, we obtain crucial bounds on the errors that arise by simplifying the factors of $\mathcal{G}$ into more tractable expressions. A list of all the symbols introduced during these computations can be found in $\S \mathrm{H}$.

In order to develop the main story in a reasonably streamlined fashion that focuses on the key ideas, we have chosen to transfer many of the underlying estimates and algebraic manipulations to the appendices. In a sense, these appendices now tell a story of their own, leading up to $\S \mathrm{F}-\mathrm{G}$ which contain the proofs of the estimates formulated in $\S 7$. These computations are essential but also tedious at times, which makes these appendices rather lengthy. We emphasize once again that the results are not only useful for the present paper. Indeed, they form the backbone for the sequel studies [31,32].

\section{Main Results}

The main results of this paper concern adaptive-grid discretizations of the scalar PDE

$$
u_{t}=u_{x x}+g(u) .
$$

In particular, we fix $h>0$ and consider a sequence of gridpoints that we index somewhat unconventionally by $h \mathbb{Z}$, in order to highlight the scale of their spatial distribution and prevent cumbersome coordinate transformations.

For any $j \in \mathbb{Z}$, we write $x_{j h}(t)$ for the time-dependent location of the relevant gridpoint and $U_{j h}(t)$ for the associated function value, which ideally should be a close approximation for $u\left(x_{j h}(t), t\right)$. The adaptive scheme that we study here can be formulated as

$$
\begin{aligned}
\dot{U}_{j h}(t)=\left[\frac{U_{(j+1) h}(t)-U_{(j-1) h}(t)}{x_{(j+1) h}(t)-x_{(j-1) h}(t)}\right] \dot{x}_{j h}(t) \\
\quad+\frac{2}{x_{(j+1) h}(t)-x_{(j-1) h}(t)} \\
\times\left[\frac{U_{(j-1) h}(t)-U_{j h}(t)}{x_{j h}(t)-x_{(j-1) h}(t)}+\frac{U_{(j+1) h}(t)-U_{j h}(t)}{x_{(j+1) h}(t)-x_{j h}(t)}\right]+g\left(U_{j h}(t)\right),
\end{aligned}
$$

in which $x(t)$ is defined implicitly by demanding that

$$
\left(x_{(j+1) h}(t)-x_{j h}(t)\right)^{2}+\left(U_{(j+1) h}(t)-U_{j h}(t)\right)^{2}=h^{2}
$$

and imposing the boundary constraint

$$
\lim _{j \rightarrow-\infty}\left[x_{j h}(t)-j h\right]=0 .
$$

Throughout the paper, we assume that the nonlinearity $g$ satisfies the following standard bistability condition. The standard example that we have in mind is the cubic $g(u)=u(1-$ $u)(u-a)$.

$(\mathrm{Hg})$ The nonlinearity $g: \mathbb{R} \rightarrow \mathbb{R}$ is $C^{3}$-smooth and has a bistable structure, in the sense that there exists a constant $0<a<1$ such that we have

$$
g(0)=g(a)=g(1)=0, \quad g^{\prime}(0)<0, \quad g^{\prime}(1)<0,
$$


together with

$$
g(u)<0 \text { for } u \in(0, a) \cup(1, \infty), \quad g(u)>0 \text { for } u \in(-\infty,-1) \cup(a, 1) .
$$

Our main goal here is to show how the implicit requirements (2.3)-(2.4) can be made explicit. In particular, we introduce the equilibrium grid

$$
\left[x_{\mathrm{eq} ; h}\right]_{j h}=j h
$$

together with the sequence space

$$
\mathcal{X}_{h}=\left\{x: h \mathbb{Z} \rightarrow \mathbb{R} \text { for which }\|x\|_{\mathcal{X}_{h}}:=\left\|x-x_{\mathrm{eq} ; h}\right\|_{\infty}=\sup _{j \in \mathbb{Z}}\left|x_{j h}-j h\right|<\infty\right\}
$$

and write

$$
x(t)=\left\{x_{j h}(t)\right\}_{j \in \mathbb{Z}} \in \mathcal{X}_{h} .
$$

We now set out to formulate a well-posed equation for the dynamics of

$$
U(t)=\left\{U_{j h}(t)\right\}_{j \in \mathbb{Z}} \in \ell^{\infty}(h \mathbb{Z} ; \mathbb{R})
$$

from which the dependence on $x$ and $\dot{x}$ has been eliminated.

As a preparation, for any $U \in \ell^{\infty}(h \mathbb{Z} ; \mathbb{R})$ we introduce the notation

$$
\partial^{+} U \in \ell^{\infty}(h \mathbb{Z} ; \mathbb{R}), \quad \partial^{-} U \in \ell^{\infty}(h \mathbb{Z} ; \mathbb{R}), \quad \partial^{0} U \in \ell^{\infty}(h \mathbb{Z} ; \mathbb{R})
$$

for the sequences

$$
\begin{aligned}
{\left[\partial^{+} U\right]_{j h} } & =h^{-1}\left[U_{(j+1) h}-U_{j h}\right], \\
{\left[\partial^{-} U\right]_{j h} } & =h^{-1}\left[U_{j h}-U_{(j-1) h}\right], \\
{\left[\partial^{0} U\right]_{j h} } & =(2 h)^{-1}\left[U_{(j+1) h}-U_{(j-1) h}\right] .
\end{aligned}
$$

In addition, for any $U \in \ell^{\infty}(h \mathbb{Z} ; \mathbb{R})$ for which $\left\|\partial^{+} U\right\|_{\infty}<1$, we define the adaptive discrete derivatives

$$
\mathcal{D}^{\diamond^{ \pm}}(U) \in \ell^{\infty}(h \mathbb{Z} ; \mathbb{R}), \quad \mathcal{D}^{\diamond_{0}}(U) \in \ell^{\infty}(h \mathbb{Z} ; \mathbb{R}), \quad \mathcal{D}^{\diamond_{0}}(U) \in \ell^{\infty}(h \mathbb{Z} ; \mathbb{R})
$$

by means of the pointwise identities

$$
\begin{aligned}
\mathcal{D}^{\diamond}(U) & =\frac{\partial^{-} U}{\sqrt{1-\left(\partial^{-} U\right)^{2}}}, \\
\mathcal{D}^{\diamond+}(U) & =\frac{\partial^{+} U}{\sqrt{1-\left(\partial^{+} U\right)^{2}}}, \\
\mathcal{D}^{\diamond}(U) & =\frac{2 \partial^{0} U}{\sqrt{1-\left(\partial^{+} U\right)^{2}}+\sqrt{1-\left(\partial^{-} U\right)^{2}}}, \\
\mathcal{D}^{\diamond \diamond_{0}}(U) & =\frac{2}{h} \frac{\mathcal{D}^{\diamond}(U)-\mathcal{D}^{\diamond}(U)}{\sqrt{1-\left(\partial^{+} U\right)^{2}}+\sqrt{1-\left(\partial^{-} U\right)^{2}}} .
\end{aligned}
$$

We also introduce the Heaviside sequence $H \in \ell^{\infty}(h \mathbb{Z} ; \mathbb{R})$ that has

$$
H_{j h}=\left\{\begin{array}{l}
1 \text { for } j \geq 0, \\
0 \text { for } j<0 .
\end{array}\right.
$$


Finally, we introduce the formal expression

$$
\mathcal{Q}_{j h}(U)=\sum_{j^{\prime}<j}\left[\ln \left[1+\mathcal{D}_{j^{\prime} h}^{\diamond_{+}}(U) \mathcal{D}_{\left(j^{\prime}+1\right) h}^{\diamond_{0}}(U)\right]-\ln \left[1+\mathcal{D}_{j^{\prime} h}^{\diamond_{+}}(U) \mathcal{D}_{j^{\prime} h}^{\diamond_{0}}(U)\right]\right],
$$

together with

$$
\mathcal{Y}_{j h}(U)=-\exp \left[-\mathcal{Q}_{j h}(U)\right] h \sum_{j^{\prime}<j} \frac{\mathcal{D}_{j^{\prime} h}^{\diamond_{+}}(U) \exp \left[\mathcal{Q}_{j^{\prime} h}(U)\right]}{1+\mathcal{D}_{j^{\prime} h}^{\diamond^{+}}(U) \mathcal{D}_{j^{\prime} h}^{\diamond_{0}}(U)} \partial^{+}\left[\mathcal{D}^{\diamond_{0}}(U)+g(U)\right]_{j^{\prime} h} .
$$

Upon imposing a summability condition on $U$ it is possible to show that these expressions are well-defined.

Lemma 2.1 (see \$4) Suppose that $(\mathrm{Hg})$ is satisfied, fix $h>0$ and consider any $U \in$ $\ell^{\infty}(h \mathbb{Z} ; \mathbb{R})$ for which $U-H \in \ell^{2}(h \mathbb{Z} ; \mathbb{R})$ and $\left\|\partial^{+} U\right\|_{\infty}<1$. Then the sequences

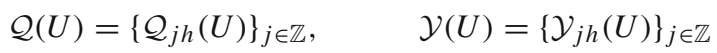

are both well-defined and we have

$$
\mathcal{Q}(U) \in \ell^{\infty}(h \mathbb{Z} ; \mathbb{R}), \quad \mathcal{Y}(U) \in \ell^{\infty}(h \mathbb{Z} ; \mathbb{R}) .
$$

Our first main result shows that the expression $\mathcal{Y}(U)$ can be used to replace the $\dot{x}$ term appearing in (2.2). The remaining terms involving $x$ can be eliminated using the implicit relation (2.3).

Theorem 2.2 (see §4) Suppose that $(\mathrm{Hg})$ is satisfied and fix $h>0$ together with $T>0$. Consider two functions

$$
x:[0, T] \mapsto \mathcal{X}_{h}, \quad U:[0, T] \mapsto \ell^{\infty}(h \mathbb{Z} ; \mathbb{R})
$$

that satisfy the following properties.

(a) We have the inclusions

$$
\begin{aligned}
& t \mapsto U(t)-H \quad \in C^{1}\left([0, T] ; \ell^{2}(h \mathbb{Z} ; \mathbb{R})\right), \\
& t \mapsto x(t)-x_{\mathrm{eq} ; h} \in C^{1}\left([0, T] ; \ell^{\infty}(h \mathbb{Z} ; \mathbb{R})\right) .
\end{aligned}
$$

(b) For every $j \in \mathbb{Z}$ and $0 \leq t \leq T$ we have the identity

$$
x_{(j+1) h}(t)-x_{j h}(t)=\sqrt{h^{2}-\left(U_{(j+1) h}(t)-U_{j h}(t)\right)^{2}} .
$$

(c) For every $0 \leq t \leq T$ we have the limit

$$
\lim _{j \rightarrow-\infty}\left[x_{j h}(t)-j h\right]=0 .
$$

(d) For every $0 \leq t \leq T$ we have the strict inequality

$$
\inf _{j \in \mathbb{Z}}\left[x_{(j+1) h}(t)-x_{j h}(t)\right]>0 .
$$

(e) For every $0 \leq t \leq T$ and $j \in \mathbb{Z}$ the identity (2.2) holds.

Then the function $U$ satisfies the system

$$
\dot{U}(t)=\mathcal{D}^{\diamond_{0}}(U(t)) \mathcal{Y}(U(t))+\mathcal{D}^{\diamond_{0}}(U(t))+g(U(t))
$$

for all $0 \leq t \leq T$. 
Conversely, once a solution to (2.25) has been obtained, it is possible to construct a solution to the full problem (2.2). Indeed, the following result shows how the position of the gridpoints can be recovered from $U(t)$.

Theorem 2.3 (see §4) Suppose that $(\mathrm{Hg})$ is satisfied and fix $h>0$ together with $T>0$. Consider a function $U:[0, T] \rightarrow \ell^{\infty}(h \mathbb{Z} ; \mathbb{R})$ that satisfies the following properties.

(a') We have the inclusion

$$
t \mapsto U(t)-H \in C^{1}\left([0, T] ; \ell^{2}(h \mathbb{Z} ; \mathbb{R})\right) .
$$

(b') The strict inequality

$$
\left\|\partial^{+} U(t)\right\|_{\infty}<1
$$

holds for every $t \in[0, T]$.

(c') For every $t \in[0, T]$ the identity

$$
\dot{U}(t)=\mathcal{D}^{\diamond_{0}}(U(t)) \mathcal{Y}(U(t))+\mathcal{D}^{\diamond_{0}}(U(t))+g(U(t))
$$

is satisfied.

Then upon writing

$$
x_{j h}(t)=j h-\sum_{j^{\prime}<j} \frac{\left(U_{\left(j^{\prime}+1\right) h}(t)-U_{j^{\prime} h}(t)\right)^{2}}{\sqrt{h^{2}-\left(U_{\left(j^{\prime}+1\right) h}(t)-U_{j^{\prime} h}(t)\right)^{2}}+h}
$$

the properties (a), (b), (c), (d) and (e) from Proposition 2.2 are all satisfied.

We conclude our general analysis of the full problem (2.2) by showing that the reduced system (2.28) is well-posed in an appropriate sense. Indeed, we establish the following shortterm existence result for a class of summable initial conditions. We remark that the restriction (2.30) on the initial condition is a natural and unavoidable consequence of the requirement (2.3).

Proposition 2.4 (see §8) Suppose that $(\mathrm{Hg})$ is satisfied and fix $h>0$. Consider any $U^{0} \in$ $\ell^{\infty}(h \mathbb{Z} ; \mathbb{R})$ for which $U^{0}-H \in \ell^{2}(h \mathbb{Z} ; \mathbb{R})$ and for which

$$
\left\|\partial^{+} U^{0}\right\|_{\infty}<1 \text {. }
$$

Then there exists $\delta_{T}>0$ and a function $U:\left[0, \delta_{T}\right] \rightarrow \ell^{\infty}(h \mathbb{Z} ; \mathbb{R})$ that has $U(0)=U^{0}$ and that satisfies the properties ( $\left.a^{\prime}\right),\left(b^{\prime}\right)$ and (c') from Theorem 2.3 with $T=\delta_{T}$.

We do not know whether it is possible to obtain global well-posedness results for (2.28). Even on bounded domains, questions of this type are very delicate. Indeed, results stating that mesh points do not collide have only become available very recently [26]. However, this analysis features a very specific discretization scheme that satisfies a coercivity condition on the meshing function.

\section{Setup and Notation}

In this section we introduce the basic symbols and sequence spaces that are used throughout the entire paper. We start in $\$ 3.1$ by providing some basic identities concerning discrete differentiation and integration. We proceed in $\$ 3.2$ by introducing several convenient shorthands 
for the gridpoint spacing functions $\sqrt{1-\left(\partial^{ \pm} U\right)^{2}}$ and formulating several useful identities for their discrete derivatives. This allows us to obtain expressions that are uniform in $h$ for the derivative operator $\mathcal{D}^{\diamond_{0}}$ defined in (2.14) and the terms appearing in (2.16)-(2.17). We continue in $\$ 3.3$ by introducing several norms on sequence spaces that will be used to formulate our estimates. Finally, in $\S 3.4$, we introduce the affine spaces $\Omega_{h ; \kappa}$ that will contain the solutions $U$ that are described in the main results formulated in $\S 2$.

\subsection{Discrete Calculus}

For any sequence $a \in \ell^{\infty}(h \mathbb{Z} ; \mathbb{R})$ we introduce the notation $T^{ \pm} a \in \ell^{\infty}(h \mathbb{Z} ; \mathbb{R})$ to refer to the translated sequences

$$
\left[T^{+} a\right]_{j h}=a_{(j+1) h}, \quad\left[T^{-} a\right]_{j h}=a_{(j-1) h} .
$$

In addition, we introduce the notation $S^{ \pm} a \in \ell^{\infty}(h \mathbb{Z} ; \mathbb{R})$ and $P^{ \pm} a \in \ell^{\infty}(h \mathbb{Z} ; \mathbb{R})$ to refer to the sum and product sequences

$$
S^{ \pm} a=\frac{1}{2}\left(a+T^{ \pm} a\right), \quad P^{ \pm} a=a T^{ \pm} a .
$$

Writing

$$
\left[\partial^{(2)} a\right]_{j h}=\frac{1}{h}\left[\partial^{+} a-\partial^{-} a\right]_{j h}=\frac{1}{h^{2}}\left[a_{(j+1) h}+a_{(j-1) h}-2 a_{j h}\right],
$$

we point out the basic identities

$$
\partial^{(2)} a=\partial^{+} \partial^{-} a, \quad \partial^{+} \partial^{0} a=S^{+}\left[\partial^{(2)} a\right] .
$$

Consider two sequences $a \in \ell^{\infty}(h \mathbb{Z} ; \mathbb{R})$ and $b \in \ell^{\infty}(h \mathbb{Z} ; \mathbb{R})$. One may compute

$$
\begin{aligned}
\partial^{+}[a b] & =\partial^{+} a T^{+} b+a \partial^{+} b, \\
\partial^{0}[a b] & =\partial^{0} a T^{+} b+T^{-} a \partial^{0} b, \\
\partial^{-}[a b] & =\left[\partial^{-} a\right] b+\left[T^{-} a\right] \partial^{-} b,
\end{aligned}
$$

which yields

$$
\begin{aligned}
\partial^{(2)}[a b] & =\frac{1}{h}\left[\partial^{+}[a b]-\partial^{-}[a b]\right] \\
& =\left(\partial^{(2)} a\right) b+\partial^{+} a \partial^{+} b+\partial^{-} a \partial^{-} b+a \partial^{(2)} b .
\end{aligned}
$$

In addition, if $b_{j h} \neq 0$ for all $j \in \mathbb{Z}$ then we have

$$
\partial^{+}\left[\frac{a}{b}\right]=\frac{b \partial^{+} a-a \partial^{+} b}{P^{+} b} .
$$

We often use the symmetrized versions

$$
\begin{aligned}
\partial^{+}[a b] & =\partial^{+} a S^{+} b+S^{+} a \partial^{+} b, \\
\partial^{+}\left[\frac{a}{b}\right] & =\frac{S^{+} b \partial^{+} a}{P^{+} b}-\frac{S^{+} a \partial^{+} b}{P^{+} b} .
\end{aligned}
$$

For any sequence $a \in \ell^{1}(h \mathbb{Z} ; \mathbb{R})$, we define two new sequences

$$
\sum_{-; h} a \in \ell^{\infty}(h \mathbb{Z} ; \mathbb{R}), \quad \sum_{+; h} a \in \ell^{\infty}(h \mathbb{Z} ; \mathbb{R})
$$


by writing

$$
\begin{aligned}
& {\left[\sum_{-; h} a\right]_{j h}=h \sum_{k>0} a_{(j-k) h},} \\
& {\left[\sum_{+; h} a\right]_{j h}=h \sum_{k>0} a_{(j+k) h} .}
\end{aligned}
$$

This notation is inspired by the integral operator $\left[\int_{-} f\right](\theta)=\int_{-\infty}^{\theta} f\left(\theta^{\prime}\right) d \theta^{\prime}$ introduced in $\S 1$. Using the fact that $\lim _{k \rightarrow \pm \infty} a_{k h}=0$, one can read-off the identities

$$
\begin{aligned}
\partial^{+}\left[\sum_{-; h} a\right]_{j h} & =a_{j h}, \\
\partial^{-}\left[\sum_{+; h} a\right]_{j h} & =-a_{j h} .
\end{aligned}
$$

Finally, consider two sequences $a \in \ell^{2}(h \mathbb{Z} ; \mathbb{R})$ and $b \in \ell^{2}(h \mathbb{Z} ; \mathbb{R})$. Since $a b \in \ell^{1}(h \mathbb{Z} ; \mathbb{R})$, we may exploit (3.11) together with the identity

$$
\partial^{+}\left[a T^{-} b\right]=\left[\partial^{+} a\right] b+a \partial^{+}\left[T^{-} b\right]=b \partial^{+} a+a \partial^{-} b
$$

to obtain the discrete summation-by-parts formula

$$
\sum_{-; h} b \partial^{+} a=a T^{-} b-\sum_{-; h} a \partial^{-} b .
$$

In addition, we see that

$$
h \partial^{+}\left[a T^{-} b\right]=b\left(T^{+} a-a\right)+a\left(b-T^{-} b\right)=2\left(b S^{+} a-a S^{-} b\right),
$$

which gives a second summation-by-parts formula

$$
\sum_{-; h} b S^{+} a=\frac{1}{2} h a T^{-} b+\sum_{-; h} a S^{-} b .
$$

\subsection{Discrete Derivatives}

Our goal here is to introduce some notation to ease computations involving the discrete derivatives (2.14). Turning to the denominators, we first define the expressions

$$
\begin{aligned}
r_{U}^{+} & =\sqrt{1-\left(\partial^{+} U\right)^{2}}, \\
r_{U}^{-} & =\sqrt{1-\left(\partial^{-} U\right)^{2}}, \\
r_{U}^{0} & =\frac{1}{2} \sqrt{1-\left(\partial^{+} U\right)^{2}}+\frac{1}{2} \sqrt{1-\left(\partial^{-} U\right)^{2}} \\
& =\frac{1}{2}\left[r_{U}^{+}+r_{U}^{-}\right],
\end{aligned}
$$

which are all related to the spacing of the gridpoints. These allow us to rewrite the discrete first derivatives in (2.14) as

$$
\mathcal{D}^{\diamond \pm}(U)=\frac{\partial^{ \pm} U}{r_{U}^{ \pm}}, \quad \mathcal{D}^{\diamond}(U)=\frac{\partial^{0} U}{r_{U}^{0}} .
$$


Notice that

$$
T^{+} r_{U}^{-}=r_{U}^{+}, \quad T^{-} r_{U}^{+}=r_{U}^{-},
$$

which directly implies

$$
\frac{r_{U}^{+}-r_{U}^{-}}{h}=\partial^{-} r_{U}^{+}=\partial^{+} r_{U}^{-}
$$

Using Lemma C.1, this allows us to compute the first derivatives

$$
\begin{aligned}
& \partial^{+} r_{U}^{-}=-\mathcal{D}^{\diamond 0}(U) \partial^{(2)} U, \\
& \partial^{+} r_{U}^{0}=-S^{+}\left[\mathcal{D}^{\diamond}(U) \partial^{(2)} U\right] .
\end{aligned}
$$

Before turning to the function $\mathcal{D}^{\diamond \diamond_{0}}$ appearing in (2.14), we introduce the mixed second discrete derivatives

$$
\begin{aligned}
\mathcal{D}^{\diamond_{0} ;+}(U) & =\partial^{+} \mathcal{D}^{\diamond_{0}}(U), \\
\mathcal{D}^{\diamond_{-} ;+}(U) & =\partial^{+} \mathcal{D}^{\diamond}(U), \\
\mathcal{D}^{\diamond_{+} ;+}(U) & =\partial^{+} \mathcal{D}^{\diamond+}(U) .
\end{aligned}
$$

In order to isolate the second derivatives, which play a crucial role in our estimates, we introduce the expressions

$$
\begin{aligned}
& \mathcal{I}_{0}^{\diamond_{-} ;+}(U)=\frac{1}{r_{U}^{+}}\left[1+\mathcal{D}^{\diamond_{-}}(U) \mathcal{D}^{\diamond_{0}}(U)\right], \\
& \mathcal{I}_{0}^{\diamond_{0} ;+}(U)=\frac{1}{2 T^{+} r_{U}^{0}}\left[1+\mathcal{D}^{\diamond_{0}}(U) \mathcal{D}^{\diamond_{0}}(U)\right], \\
& \mathcal{I}_{s}^{\diamond_{0} ;+}(U)=\frac{1}{2 T^{+} r_{U}^{0}}\left[1+\mathcal{D}^{\diamond_{0}}(U) T^{+}\left[\mathcal{D}^{\diamond_{0}}(U)\right]\right]
\end{aligned}
$$

and note that Lemma C.3 allows us to write

$$
\begin{aligned}
& \mathcal{D}^{\diamond_{-} ;+}(U)=\mathcal{I}_{0}^{\diamond_{-} ;+}(U) \partial^{(2)} U, \\
& \mathcal{D}^{\diamond_{0} ;+}(U)=\mathcal{I}_{0}^{\diamond_{0} ;+}(U) \partial^{(2)} U+\mathcal{I}_{s}^{\diamond_{0} ;+}(U) T^{+}\left[\partial^{(2)} U\right] .
\end{aligned}
$$

Furthermore, the basic identities

$$
T^{+} \mathcal{D}^{\diamond}(U)=\mathcal{D}^{\diamond}(U), \quad T^{-} \mathcal{D}^{\diamond+}(U)=\mathcal{D}^{\diamond}(U),
$$

allow us to observe that

$$
\mathcal{D}^{\diamond_{-} ;+}(U)=\frac{\mathcal{D}^{{ }^{+}}(U)-\mathcal{D}^{\diamond_{-}}(U)}{h}, \quad \mathcal{D}^{\diamond_{+} ;+}(U)=T^{+} \mathcal{D}^{\diamond_{-} ;+}(U) .
$$

In particular, we arrive at the convenient identification

$$
\mathcal{D}^{\diamond_{0}}(U)=\frac{1}{r_{U}^{0}} \mathcal{D}^{\diamond_{-} ;+}(U) .
$$

Finally, we introduce the third mixed discrete derivative

$$
\mathcal{D}^{\diamond \diamond_{0} ;+}(U)=\partial^{+}\left[\mathcal{D}^{\diamond \diamond_{0}}(U)\right]
$$


which will play a role when taking the discrete derivative of (2.25). As before, we need to separate out the derivatives of order two and higher. To this end, we introduce the expressions

$$
\begin{aligned}
& \mathcal{I}_{0 s}^{\diamond_{0} ;+}(U)=\frac{1}{2 r_{U}^{+} P^{+} r_{U}^{0}} \mathcal{D}^{\diamond_{0}}(U) T^{+}\left[1+\mathcal{D}^{\diamond-}(U) \mathcal{D}^{\diamond_{0}}(U)\right]
\end{aligned}
$$

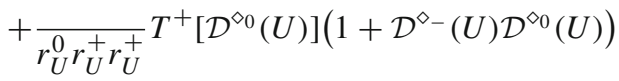

$$
\begin{aligned}
& +\frac{1}{2 r_{U}^{+} P^{+} r_{U}^{0}} \mathcal{D}^{\diamond}(U)\left(1+\mathcal{D}^{\diamond}(U)^{2}\right), \\
& \mathcal{I}_{s s}^{\diamond_{0} ;+}(U)=\frac{2 r_{U}^{0}+T^{+} r_{U}^{+}}{2 P^{+} r_{U}^{0} P^{+} r_{U}^{+}} T^{+}\left[\mathcal{D}^{\diamond_{0}}(U)\left(1+\mathcal{D}^{\diamond}(U) \mathcal{D}^{\diamond_{0}}(U)\right)\right] \\
& +\frac{1}{2 r_{U}^{+} P^{+} r_{U}^{0}} \mathcal{D}^{\diamond-}(U)\left(1+\mathcal{D}^{\diamond_{0}}(U) T^{+}\left[\mathcal{D}^{\diamond_{0}}(U)\right]\right), \\
& \mathcal{I}_{+}^{\diamond_{0} ;+}(U)=\frac{1}{r_{U}^{0} r_{U}^{+}}\left(1+\mathcal{D}^{\diamond}(U) \mathcal{D}^{\diamond_{0}}(U)\right),
\end{aligned}
$$

which all only feature first derivatives. Lemma C.4 shows that $\mathcal{D}^{\diamond \diamond_{0} ;+}(U)$ can be written as the sum of the two components

$$
\begin{aligned}
& \mathcal{D}_{a}^{\diamond_{0} ;+}(U)=\mathcal{I}_{+}^{\diamond_{0} ;+}(U) \partial^{+} \partial^{(2)} U \\
& \mathcal{D}_{b}^{\diamond \diamond_{0} ;+}(U)=\mathcal{I}_{0 s}^{\diamond_{0} ;+}(U) \partial^{(2)} U T^{+}\left[\partial^{(2)} U\right]+\mathcal{I}_{s s}^{\diamond_{0} ;+}(U) T^{+}\left[\partial^{(2)} U\right] T^{+}\left[\partial^{(2)} U\right] .
\end{aligned}
$$

\subsection{Sequence Spaces}

Our main concern here is to introduce the sequence spaces on which the nonlinear terms in (2.25) can be conveniently estimated in a fashion that is uniform in $h>0$. Special care needs to be taken to ensure that the square roots in the expressions (2.14) are well-defined. For any $h>0$, we first introduce the Hilbert space $\ell_{h}^{2}$ that is equal to $\ell^{2}(h \mathbb{Z} ; \mathbb{R})$ as a set, but is equipped with the rescaled inner product

$$
\langle V, W\rangle_{\ell_{h}^{2}}=h \sum_{j \in \mathbb{Z}} V_{j h} W_{j h}
$$

that compensates for the gridpoint density. In particular, for $V \in \ell_{h}^{2}$ we have

$$
\|V\|_{\ell_{h}^{2}}^{2}=h \sum_{j \in \mathbb{Z}} V_{j h}^{2} .
$$

For convenience, we also introduce the alternative notation

$$
\ell_{h}^{\infty}=\left\{V: h \mathbb{Z} \rightarrow \mathbb{R} \text { for which }\|V\|_{\ell_{h}^{\infty}}:=\sup _{j \in \mathbb{Z}}\left|V_{h j}\right|<\infty\right\}
$$

for the usual set $\ell^{\infty}(h \mathbb{Z} ; \mathbb{R})$ with the supremum norm. For any $V \in \ell_{h}^{2}$, it is clear that also $V \in \ell_{h}^{\infty}$ and that we have the bound

$$
\|V\|_{\ell_{h}^{\infty}} \leq h^{-1 / 2}\|V\|_{\ell_{h}^{2}} .
$$

In order to reduce the length of our expressions, we introduce the higher order norms

$$
\|V\|_{\ell_{h}^{2 ; 1}}=\|V\|_{\ell_{h}^{2}}+\left\|\partial^{+} V\right\|_{\ell_{h}^{2}}
$$




$$
\begin{aligned}
& \|V\|_{\ell_{h}^{2 ; 2}}=\|V\|_{\ell_{h}^{2}}+\left\|\partial^{+} V\right\|_{\ell_{h}^{2}}+\left\|\partial^{+} \partial^{+} V\right\|_{\ell_{h}^{2}}, \\
& \|V\|_{\ell_{h}^{2 ; 3}}=\|V\|_{\ell_{h}^{2}}+\left\|\partial^{+} V\right\|_{\ell_{h}^{2}}+\left\|\partial^{+} \partial^{+} V\right\|_{\ell_{h}^{2}}+\left\|\partial^{+} \partial^{+} \partial^{+} V\right\|_{\ell_{h}^{2}},
\end{aligned}
$$

together with

$$
\begin{aligned}
& \|V\|_{\ell_{h}^{\infty ; 1}}=\|V\|_{\ell_{h}^{\infty}}+\left\|\partial^{+} V\right\|_{\ell_{h}^{\infty}}, \\
& \|V\|_{\ell_{h}^{\infty ; 2}}=\|V\|_{\ell_{h}^{\infty}}+\left\|\partial^{+} V\right\|_{\ell_{h}^{\infty}}+\left\|\partial^{+} \partial^{+} V\right\|_{\ell_{h}^{\infty}} .
\end{aligned}
$$

We caution the reader that for fixed $h>0$, these norms are equivalent to the norms on $\ell_{h}^{2}$ respectively $\ell_{h}^{\infty}$. However, the $h$-scalings included in the derivatives (2.12) and hence inherited by (3.34)-(3.35) are essential to allow us to conveniently formulate estimates that are uniform in $h>0$. This will turn out to be crucial for the bifurcation arguments in [32].

\subsection{The Solution Space $\boldsymbol{\Omega}_{h ; \kappa}$}

In general, we cannot expect solutions to (2.25) that connect the two stable equilibria of $g$ to be contained in $\ell_{h}^{2}$. This is reflected by the presence of the Heaviside function $H$ in the inclusions (2.21) and (2.26). However, we have $\left\|\partial^{+} H\right\|_{\ell_{h}^{\infty}}=h^{-1}$, which makes this function unsuitable for the bifurcation arguments used in this series of papers.

In order to repair this problem and smoothen out the transition between $U=0$ and $U=1$, we pick a function $U_{\mathrm{ref} ; *} \in C^{2}(\mathbb{R},[0,1])$ for which we have the identities

$$
U_{\text {ref } ; *}(\tau)= \begin{cases}0 & \text { for all } \tau \leq-2 \\ 1 & \text { for all } \tau \geq 2\end{cases}
$$

and for which the bounds

$$
0 \leq U_{\mathrm{ref} ; *}^{\prime}(\tau)<1, \quad\left|U_{\mathrm{ref} ; *}^{\prime \prime}(\tau)\right|<1
$$

hold for all $\tau \in \mathbb{R}$. For any $\kappa>0$ we subsequently write

$$
U_{\text {ref } ; \kappa}(\tau)=U_{\text {ref;* }}(\kappa \tau)
$$

together with

$$
\begin{aligned}
\mathcal{V}_{h ; \kappa}= & \left\{V \in \ell_{h}^{2}:\|V\|_{\ell_{h}^{2 ; 2}}+\|V\|_{\ell_{h}^{\infty}}+\left\|\partial^{+} \partial^{+} V\right\|_{\ell_{h}^{\infty}}<\frac{1}{2} \kappa^{-1}\right. \\
& \text { and } \left.\left\|\partial^{+} V\right\|_{\infty}<1-2 \kappa\right\} .
\end{aligned}
$$

As a consequence of the estimate (3.33), we see that $\mathcal{V}_{h ; \kappa}$ is an open subset of $\ell_{h}^{2}$.

Combining these two definitions allows us to introduce the sets

$$
\Omega_{h ; \kappa}=U_{\text {ref } ; \kappa}(h \mathbb{Z})+\mathcal{V}_{h ; \kappa} \subset \ell_{h}^{\infty} .
$$

Our three main results highlight the important role that these sets $\Omega_{h ; \kappa}$ will play in the sequel. Indeed, the initial conditions referenced in the well-posedness result Proposition 2.4 can all be taken from such a set. In addition, we obtain a-priori bounds on almost all the terms that appear in the discrete derivatives defined in $\$ 3.2$. The one exception is the third derivative $\partial^{+} \partial^{(2)} U$, which will play a special role in our estimates. 
Proposition 3.1 (see §B) Fix $h>0$ and $0<\kappa<\frac{1}{12}$. Then for any $U \in \Omega_{h ; \kappa}$, we have the bound

$$
\|U\|_{\ell_{h}^{\infty}}+\left\|\partial^{+} U\right\|_{\ell_{h}^{2}}+\left\|\partial^{+} \partial^{+} U\right\|_{\ell_{h}^{2}}+\left\|\partial^{+} \partial^{+} U\right\|_{\ell_{h}^{\infty}}<\kappa^{-1}
$$

together with

$$
\left\|\partial^{+} U\right\|_{\ell_{h}^{\infty}}<1-\kappa
$$

In addition, we have

$$
\|g(U)\|_{\ell_{h}^{2}} \leq 4\left[\sup _{|u| \leq \kappa^{-1}}\left|g^{\prime}(u)\right|\right] \kappa^{-1}
$$

Proposition 3.2 (see §B) Fix $h>0$ and consider any $U \in \ell_{h}^{\infty}$ for which $\left\|\partial^{+} U\right\|_{\infty}<1$ and $U-H \in \ell_{h}^{2}$. Then there exist $\epsilon_{0}>0$ and $\kappa_{0}>0$ so that for any $\widetilde{U} \in \ell_{h}^{\infty}$ that has $\|\widetilde{U}-U\|_{\ell_{h}^{2}}<\epsilon_{0}$, we have

$$
\widetilde{U} \in \Omega_{h ; \kappa}
$$

for all $0<\kappa<\kappa_{0}$.

Proposition 3.3 (see $\S \mathrm{B})$ Consider any $u \in C(\mathbb{R} ; \mathbb{R})$ for which we have the inclusions

$$
u^{\prime} \in H^{2}, \quad u-U_{\text {ref; } *} \in L^{2},
$$

together with the bound $\left\|u^{\prime}\right\|_{L^{\infty}}<1$. Then there exist $\epsilon_{0}>0$ and $\kappa>0$ so that for any $0<h<1$ and any $v \in H^{1}$ that has

$$
\|v\|_{H^{1}}+h^{-1 / 2}\left\|\partial^{+} v\right\|_{H^{1}}<\epsilon_{0},
$$

we have

$$
[u+v](\vartheta+h \mathbb{Z}) \in \Omega_{h ; \kappa}
$$

for all $\vartheta \in[0, h]$.

\subsection{Convention}

Throughout the remainder of this paper, we use the convention that primed constants (such as $C_{1}^{\prime}, C_{2}^{\prime}$ etc) that appear in proofs are positive and depend only on $\kappa$ and the nonlinearity $g$, unless explicitly stated otherwise.

\section{Gridpoint Behaviour}

In this section we derive the reduced equation (2.25) by analyzing the function $\mathcal{Y}(U)$ defined in (2.17) and showing that the speed of the gridpoints satisfies

$$
\dot{x}=\mathcal{Y}(U) .
$$

In particular, we establish Lemma 2.1 together with Theorems 2.2 and 2.3 . In essence, we develop a discrete version of the arguments described in $\S 1$ to pass from (1.19) to (1.22). 
In order to clean up the expressions (2.16)-(2.17), we introduce the functions

$$
\tilde{p}(U)=\frac{1}{1+\mathcal{D}^{\diamond}+(U) \mathcal{D}^{\diamond}(U)}, \quad p(U)=\mathcal{D}^{\diamond}(U) \tilde{p}(U),
$$

together with

$$
q(U)=h^{-1} \ln \left[1+h p(U) \mathcal{D}^{\diamond_{0} ;+}(U)\right] .
$$

In addition, we introduce the functions

$$
\mathcal{Z}^{+}(U)=\exp [\mathcal{Q}(U)], \quad \mathcal{Z}^{-}(U)=\exp [-\mathcal{Q}(U)] .
$$

Our first main result states that these expressions are well-defined, allowing us to obtain reasonably compact expressions for $\mathcal{Y}(U)$ and its discrete derivative.

Proposition 4.1 Assume that $(\mathrm{Hg})$ is satisfied and fix $0<\kappa<\frac{1}{12}$ and $h>0$. For any $U \in \Omega_{h ; \kappa}$, we have the inclusions

$$
\widetilde{p}(U) \in \ell^{\infty}(h \mathbb{Z} ; \mathbb{R}), \quad p(U) \in \ell^{2}(h \mathbb{Z} ; \mathbb{R}), \quad q(U) \in \ell^{1}(h \mathbb{Z} ; \mathbb{R}),
$$

together with

$$
\mathcal{Q}(U) \in \ell^{\infty}(h \mathbb{Z} ; \mathbb{R}), \quad \mathcal{Z}^{ \pm}(U) \in \ell^{\infty}(h \mathbb{Z} ; \mathbb{R}), \quad \mathcal{Y}(U) \in \ell^{\infty}(h \mathbb{Z} ; \mathbb{R}) .
$$

In addition, we have the identity

$$
\mathcal{Q}(U)=\sum_{-; h} q(U)
$$

together with

$$
\begin{aligned}
\mathcal{Y}(U)= & -\mathcal{Z}^{-}(U) \sum_{-; h} p(U) \mathcal{Z}^{+}(U) \partial^{+}\left[\mathcal{D}^{\diamond_{0}}(U)+g(U)\right], \\
\partial^{+} \mathcal{Y}(U)= & -T^{+}\left[\mathcal{Z}^{-}(U)\right] p(U) \mathcal{D}^{\diamond_{0} ;+}(U) S^{+}\left[\mathcal{Z}^{+}(U) \mathcal{Y}(U)\right] \\
& -S^{+}\left[\mathcal{Z}^{-}(U)\right] p(U) \mathcal{Z}^{+}(U) \partial^{+}\left[\mathcal{D}^{\diamond_{0}}(U)+g(U)\right] .
\end{aligned}
$$

Finally, for every $U \in \Omega_{h ; \kappa}$ we have the limit

$$
\lim _{j \rightarrow-\infty} \mathcal{Y}_{j h}(U)=0 .
$$

Our second main result shows that we indeed have $\dot{x}=\mathcal{Y}(U)$, irrespective of whether the full equation (2.2) or the reduced system (2.25) is satisfied. We remark that the two conditions (a) and (b) will turn out to be equivalent, but this is established later.

Proposition 4.2 Suppose that $(\mathrm{Hg})$ is satisfied. Consider a function $U:[0, T] \rightarrow \ell_{h}^{\infty}$ for which $U-H \in C^{1}\left([0, T] ; \ell_{h}^{2}\right)$ and

$$
\left\|\partial^{+} U(t)\right\|_{\ell_{h}^{\infty}}<1
$$

for all $0 \leq t \leq T$. Write

$$
\begin{aligned}
x(t) & =x_{\mathrm{eq} ; h}+\sum_{-; h}\left(r_{U(t)}^{+}-1\right) \\
& =x_{\mathrm{eq} ; h}-\sum_{-; h} \frac{\left(\partial^{+} U(t)\right)^{2}}{r_{U(t)}^{+}+1} .
\end{aligned}
$$

Suppose furthermore that at least one of the following two conditions holds. 
(a) The function $U$ satisfies (2.25) on $[0, T]$.

(b) The pair $(U, x)$ satisfies $(2.2)$ on $[0, T]$.

Then there exists $0<\kappa<\frac{1}{12}$ so that for every $0 \leq t \leq T$ we have the inclusion

$$
U(t) \in \Omega_{h ; \kappa},
$$

together with the identity

$$
\dot{x}(t)=\mathcal{Y}(U(t))
$$

\subsection{Basic Properties for $\mathcal{Z}$ and $\mathcal{Y}$}

In this subsection we show that the definitions above are well-posed. In addition, we establish some basic identities for the discrete derivatives of $\mathcal{Z}$ and $\mathcal{Y}$ that allow us to establish Proposition 4.1. We start by examining the function $q$ introduced in (4.3).

Lemma 4.3 Fix $0<\kappa<\frac{1}{12}$ and $h>0$. For any $U \in \Omega_{h ; \kappa}$ we have the identity

$$
h q(U)=\ln \left[1+\mathcal{D}^{\diamond}(U) T^{+} \mathcal{D}^{\diamond}(U)\right]-\ln \left[1+\mathcal{D}^{\diamond}(U) \mathcal{D}^{\diamond}(U)\right],
$$

together with the inequality

$$
\exp [h q(U)]=1+h p(U) \mathcal{D}^{\diamond_{0} ;+}(U) \geq \frac{1}{3} \kappa^{2} .
$$

Proof We compute

$$
\begin{aligned}
\exp [h q(U)] & =1+h p(U) \partial^{+} \mathcal{D}^{\diamond_{0}}(U) \\
& =1+\frac{\mathcal{D}^{\diamond}(U) h \partial^{+} \mathcal{D}^{\diamond_{0}}(U)}{1+\mathcal{D}^{\diamond}(U) \mathcal{D}^{\diamond_{0}}(U)} \\
& =\frac{1+\mathcal{D}^{\diamond}(U) \mathcal{D}^{\diamond_{0}}(U)+\mathcal{D}^{\diamond}(U) h \partial^{+} \mathcal{D}^{\diamond_{0}}(U)}{1+\mathcal{D}^{\diamond}(U) \mathcal{D}^{\diamond}(U)} \\
& =\frac{1+\mathcal{D}^{\diamond}(U) T^{+} \mathcal{D}^{\diamond_{0}}(U)}{1+\mathcal{D}^{\diamond}(U) \mathcal{D}^{\diamond_{0}}(U)},
\end{aligned}
$$

which directly implies (4.14). In addition, we may use Lemma D.7 to conclude

$$
\exp [h q(U)]=\frac{T^{+}\left[1+\mathcal{D}^{\diamond}-(U) \mathcal{D}^{\diamond}(U)\right]}{1+\mathcal{D}^{\diamond}+(U) \mathcal{D}^{\diamond}(U)} \geq \frac{\frac{1}{2} \kappa}{\frac{3}{2} \kappa^{-1}}=\frac{1}{3} \kappa^{2} .
$$

Lemma 4.4 Fix $0<\kappa<\frac{1}{12}$ and $h>0$. For any $U \in \Omega_{h ; \kappa}$, we have the inclusions

$$
\tilde{p}(U) \in \ell^{\infty}(h \mathbb{Z} ; \mathbb{R}), \quad p(U) \in \ell^{2}(h \mathbb{Z} ; \mathbb{R}), \quad q(U) \in \ell^{1}(h \mathbb{Z} ; \mathbb{R}),
$$

together with the identity

$$
\mathcal{Q}(U)=\sum_{-; h} q(U)
$$


Proof Recalling (4.2), we note that Lemma D.7 yields

$$
\tilde{p}(U)=\left[1+\mathcal{D}^{\diamond}(U) \mathcal{D}^{\diamond}(U)\right]^{-1} \in \ell^{\infty}(h \mathbb{Z} ; \mathbb{R}) .
$$

Together with Lemma D.5 this shows that $p(U) \in \ell^{2}(h \mathbb{Z} ; \mathbb{R})$.

Since $\frac{d}{d x}[\ln (x)]$ can be uniformly bounded on sets of the form $x \geq \frac{1}{3} \kappa^{2}>0$, the bound (4.15) implies that there exists $C_{1}^{\prime}>1$ for which

$$
|h q(U)| \leq C_{1}^{\prime} h|p(U)|\left|\mathcal{D}^{\diamond_{0} ;+}(U)\right| .
$$

Lemma D.5 implies that $\mathcal{D}_{0}^{\diamond_{0} ;+}(U) \in \ell^{2}(h \mathbb{Z} ; \mathbb{R})$, allowing us to apply Cauchy-Schwartz to conclude that $q(U) \in \ell^{1}(h \mathbb{Z} ; \mathbb{R})$. Finally, the identity (4.19) follows directly from (4.14).

Lemma 4.5 Assume that $(\mathrm{Hg})$ is satisfied and fix $0<\kappa<\frac{1}{12}$ and $h>0$. For any $U \in \Omega_{h ; \kappa}$, we have the inclusions

$$
\mathcal{Q}(U) \in \ell^{\infty}(h \mathbb{Z} ; \mathbb{R}), \quad \mathcal{Z}^{ \pm}(U) \in \ell^{\infty}(h \mathbb{Z} ; \mathbb{R}), \quad \mathcal{Y}(U) \in \ell^{\infty}(h \mathbb{Z} ; \mathbb{R}),
$$

together with the identity

$$
\mathcal{Y}(U)=-\mathcal{Z}^{-}(U) \sum_{-; h} p(U) \mathcal{Z}^{+}(U) \partial^{+}\left[\mathcal{D}^{\diamond_{0}}(U)+g(U)\right]
$$

In addition, the limit (4.9) holds.

Proof The inclusions (4.22) for $\mathcal{Q}$ and $\mathcal{Z}^{ \pm}$follow immediately from Lemma 4.4 and the definitions (4.4). The expression (4.23) follows immediately from the definition (2.17).

We note that we have the inclusions $p(U) \in \ell_{h}^{2}, \mathcal{D}^{\diamond_{0}}(U) \in \ell_{h}^{2}$ and $g(U) \in \ell_{h}^{2}$ by Lemma 4.4, Lemma D.5 and Proposition 3.1 respectively. In particular, writing

$$
\mathcal{H}(U)=p(U) \mathcal{Z}^{+}(U) \partial^{+}\left[\mathcal{D}^{\diamond_{0}}(U)+g(U)\right]
$$

we may use the fact that $\partial^{+}$is a bounded operator on $\ell^{2}(h \mathbb{Z} ; \mathbb{R})$ to conclude by CauchySchwarz that $\mathcal{H}(U) \in \ell^{1}(h \mathbb{Z} ; \mathbb{R})$. The inclusion $\mathcal{Y}(U) \in \ell^{\infty}(h \mathbb{Z} ; \mathbb{R})$ and the limit (4.9) follow directly from this.

We remark that we explicitly constructed $\mathcal{Z}^{+}(U)$ with the aim of satisfying the first identity in (4.26). Indeed, writing $Z=\mathcal{Z}^{+}$and attempting to solve this equation, we compute

$$
h \partial^{+} \ln (Z)=\ln \left(T^{+} Z\right)-\ln (Z)=\ln \left(1+h Z^{-1} \partial^{+} Z\right)=\ln \left[1+p(U) h \mathcal{D}^{\diamond ;+}(U)\right],
$$

which leads naturally to (4.4). This choice will allow us to use $\mathcal{Z}^{+}$as a discrete version of the integrating factor $\gamma^{-1}$ used in (1.20); see the proof of Lemma 4.10 below and (4.58) in particular.

Lemma 4.6 Fix $0<\kappa<\frac{1}{12}$ and $h>0$. For any $U \in \Omega_{h ; \kappa}$, we have the identities

$$
\begin{aligned}
& \partial^{+}\left[\mathcal{Z}^{+}(U)\right]=\mathcal{Z}^{+}(U) p(U) \mathcal{D}^{\diamond_{0} ;+}(U), \\
& \partial^{+}\left[\mathcal{Z}^{-}(U)\right]=-T^{+}\left[\mathcal{Z}^{-}(U)\right] p(U) \mathcal{D}^{\diamond_{0} ;+}(U) .
\end{aligned}
$$

Proof For any $U \in \ell^{\infty}(h \mathbb{Z} ; \mathbb{R})$ we observe that

$$
\partial^{+}[\exp [U]]=h^{-1} \exp [U]\left[\exp \left[T^{+}[U]-U\right]-1\right]
$$




$$
=h^{-1} \exp [U]\left[\exp \left[h \partial^{+}[U]\right]-1\right]
$$

This allows us to compute

$$
\begin{aligned}
\partial^{+} \mathcal{Z}^{+}(U) & =h^{-1} \mathcal{Z}^{+}(U)\left[\exp \left[h \partial^{+}[\mathcal{Q}(U)]\right]-1\right] \\
& =h^{-1} \mathcal{Z}^{+}(U)\left[\exp \left[\ln \left[1+h p(U) \mathcal{D}^{\diamond_{0} ;+}(U)\right]\right]-1\right] \\
& =h^{-1} \mathcal{Z}^{+}(U)\left[h p(U) \mathcal{D}^{\diamond_{0} ;+}(U)\right]
\end{aligned}
$$

which yields the first identity. Using (3.7) we compute

$$
\begin{aligned}
\partial^{+}\left[\mathcal{Z}^{-}(U)\right] & =\partial^{+}\left[\frac{1}{\mathcal{Z}^{+}(U)}\right] \\
& =-\frac{\mathcal{Z}^{+}(U) p(U) \mathcal{D}^{\diamond_{0} ;+}(U)}{P^{+} \mathcal{Z}^{+}(U)}
\end{aligned}
$$

which yields the second identity.

Lemma 4.7 Assume that $(\mathrm{Hg})$ is satisfied and fix $0<\kappa<\frac{1}{12}$ and $h>0$. For any $U \in \Omega_{h ; \kappa}$, we have the identities

$$
\begin{aligned}
\partial^{+}\left[\mathcal{Z}^{+}(U) \mathcal{Y}(U)\right] & =-p(U) \mathcal{Z}^{+}(U) \partial^{+}\left[\mathcal{D}^{\diamond_{0}}(U)+g(U)\right], \\
\partial^{+}\left[\mathcal{D}^{\diamond_{0}}(U) \mathcal{Z}^{-}(U)\right] & =\widetilde{p}(U) T^{+}\left[\mathcal{Z}^{-}(U)\right] \mathcal{D}^{\diamond_{0} ;+}(U) .
\end{aligned}
$$

Proof The first identity follows from $\mathcal{Z}^{+}(U) \mathcal{Z}^{-}(U)=1$ together with the expression (4.23) for $\mathcal{Y}(U)$. In addition, using (4.26) and (3.5) we compute

$$
\begin{aligned}
\partial^{+}\left[\mathcal{D}^{\diamond_{0}}(U) \mathcal{Z}^{-}(U)\right] & =\mathcal{D}^{\diamond_{0} ;+}(U) T^{+}\left[\mathcal{Z}^{-}(U)\right]-\mathcal{D}^{\diamond_{0}}(U) T^{+}\left[\mathcal{Z}^{-}(U)\right] p(U) \mathcal{D}^{\diamond_{0} ;+}(U) \\
& =\left(1-\mathcal{D}^{\diamond_{0}}(U) p(U)\right) T^{+}\left[\mathcal{Z}^{-}(U)\right] \mathcal{D}^{\diamond_{0} ;+}(U) .
\end{aligned}
$$

The second identity follows from the observation

$$
\mathcal{D}^{\diamond_{0}}(U) p(U)=1-\tilde{p}(U)
$$

which is a direct consequence of the definitions (4.2).

Proof of Proposition 4.1 In view of Lemma's 4.4 and 4.5, it suffices to establish the identity for $\partial^{+} \mathcal{Y}$. Exploiting (3.8), (4.26) and (4.30), we compute

$$
\begin{aligned}
\partial^{+} \mathcal{Y}(U)= & \partial^{+}\left[\mathcal{Z}^{-}(U) \mathcal{Z}^{+}(U) \mathcal{Y}(U)\right] \\
= & \partial^{+}\left[\mathcal{Z}^{-}(U)\right] S^{+}\left[\mathcal{Z}^{+}(U) \mathcal{Y}(U)\right] \\
& -S^{+}\left[\mathcal{Z}^{-}(U)\right] p(U) \mathcal{Z}^{+}(U) \partial^{+}\left[\mathcal{D}^{\diamond_{0}}(U)+g(U)\right] \\
= & -\left[T^{+}\left[\mathcal{Z}^{-}(U)\right] p(U) \mathcal{D}^{\diamond_{0} ;}(U)\right] S^{+}\left[\mathcal{Z}^{+}(U) \mathcal{Y}(U)\right] \\
& -S^{+}\left[\mathcal{Z}^{-}(U)\right] p(U) \mathcal{Z}^{+}(U) \partial^{+}\left[\mathcal{D}^{\diamond_{0}}(U)+g(U)\right],
\end{aligned}
$$

as desired. 


\subsection{Gridpoint Speed}

In this subsection we use the discrete derivatives (4.26) and (4.30) to analyze the discrete differential equations that govern the behaviour of the gridpoints. This allows us to establish Proposition 4.2 and the first three main results from $\S 2$.

Lemma 4.8 Consider the setting of Proposition 4.2, but without requiring (a) or (b) to hold. Then there exists $0<\kappa<\frac{1}{12}$ for which the inclusion

$$
U(t) \in \Omega_{h ; \kappa}
$$

holds for all $0 \leq t \leq T$. In addition, we have

$$
x-x_{\mathrm{eq} ; h} \in C^{1}\left([0, T] ; \ell_{h}^{\infty}\right),
$$

with

$$
\dot{x}=-\sum_{-; h} \mathcal{D}^{\diamond}(U) \partial^{+} \dot{U}
$$

Finally, we have the limit

$$
\lim _{j \rightarrow-\infty} \dot{x}_{j h}(t)=0
$$

for every $0 \leq t \leq T$.

Proof Since $U(t)-H$ is continuous in $\ell_{h}^{2}$, and the interval $[0, T]$ is compact, the existence of the constant $\kappa>0$ can be deduced from Proposition 3.2.

Taking a pointwise derivative, we may compute

$$
\dot{r}_{U}^{+}=-\mathcal{D}^{\diamond}(U) \partial^{+} \dot{U} .
$$

Writing

$$
p_{1}(U)=\frac{\partial^{+} U}{\sqrt{1-\left(\partial^{+} U\right)^{2}}+1}=\frac{\partial^{+} U}{r_{U}^{+}+1}
$$

and taking a pointwise derivative, we obtain the identity

$$
\begin{aligned}
\dot{p}_{1}(U) & =\frac{\partial^{+} \dot{U}}{r_{U}^{+}+1}+\frac{\partial^{+} U}{\left(r_{U}^{+}+1\right)^{2}} \mathcal{D}^{\diamond}(U) \partial^{+} \dot{U} \\
& =\frac{r_{U}^{+}+1+\partial^{+} U \mathcal{D}^{\diamond}(U)}{\left(r_{U}^{+}+1\right)^{2}} \partial^{+} \dot{U} \\
& =\frac{r_{U}^{+}+1+\left[r_{U}^{+}\right]^{-1}\left(1-\left(r_{U}^{+}\right)^{2}\right)}{\left(r_{U}^{+}+1\right)^{2}} \partial^{+} \dot{U} \\
& =\frac{1}{r_{U}^{+}\left(r_{U}^{+}+1\right)} \partial^{+} \dot{U} .
\end{aligned}
$$

The embedding $\ell_{h}^{2} \subset \ell_{h}^{\infty}$ together with the smoothness assumption on $U$ implies that

$$
\begin{aligned}
t & \mapsto p_{1}(U(t)) \in C^{1}\left([0, T] ; \ell_{h}^{2}\right), \\
t & \mapsto \partial^{+} U(t) \in C^{1}\left([0, T] ; \ell_{h}^{2}\right) .
\end{aligned}
$$


In particular, since the map

$$
\pi: \ell_{h}^{2} \times \ell_{h}^{2} \rightarrow \ell_{h}^{\infty}, \quad\left(V^{(1)}, V^{(2)}\right) \mapsto \sum_{-; h} V^{(1)} V^{(2)}
$$

is a bounded bilinear map, we see that

$$
t \mapsto \pi\left[p_{1}(U(t)), \partial^{+} U(t)\right] \in C^{1}\left([0, T] ; \ell_{h}^{\infty}\right) .
$$

Since we have

$$
x(t)=x_{\mathrm{eq} ; h}-\pi\left[p_{1}(U(t)), \partial^{+} U(t)\right]
$$

we may compute

$$
\begin{aligned}
\dot{x}(t) & =-\frac{d}{d t} \pi\left[p_{1}(U(t)), \partial^{+} U(t)\right] \\
& =-\pi\left[\dot{p}_{1}(U(t)), \partial^{+} U(t)\right]-\pi\left[p_{1}(U(t)), \partial^{+} \dot{U}(t)\right] \\
& =-\pi\left[\frac{1}{r_{U(t)}^{+}\left(r_{U(t)}^{+}+1\right)} \partial^{+} \dot{U}(t), \partial^{+} U(t)\right]-\pi\left[\frac{\partial^{+} U(t)}{r_{U(t)}^{+}+1}, \partial^{+} \dot{U}(t)\right] \\
& =-\pi\left[\left[\frac{1}{r_{U(t)}^{+}\left(r_{U(t)}^{+}+1\right)}+\frac{1}{r_{U(t)}^{+}+1}\right] \partial^{+} U(t), \partial^{+} \dot{U}(t)\right] \\
& =-\pi\left[\mathcal{D}^{\diamond}(U(t)), \partial^{+} \dot{U}(t)\right],
\end{aligned}
$$

which gives the desired expression. Finally, the limit (4.37) follows directly from the fact that $\mathcal{D}^{\diamond+}(U(t)) \in \ell_{h}^{2}$ and $\partial^{+} \dot{U}(t) \in \ell_{h}^{2}$, which means that the product is in $\ell^{1}(h \mathbb{Z} ; \mathbb{R})$.

Lemma 4.9 Consider the setting of Proposition 4.2 and suppose that (a) holds. Then we have $\dot{x}(t)=\mathcal{Y}(U(t))$ for all $0 \leq t \leq T$.

Proof Exploiting the identity (4.36), we compute

$$
\begin{aligned}
\dot{x}= & -\sum_{-; h} \mathcal{D}^{\diamond}(U) \partial^{+} \dot{U} \\
= & -\sum_{-; h} \mathcal{D}^{\diamond}(U) \partial^{+}\left[\mathcal{D}^{\diamond_{0}}(U) \mathcal{Y}(U)+\mathcal{D}^{\diamond_{0}}(U)+g(U)\right] \\
= & -\sum_{-; h} \mathcal{D}^{\diamond_{+}}(U) \partial^{+}\left[\mathcal{D}^{\diamond_{0}}(U) \mathcal{Z}^{-}(U) \mathcal{Z}^{+}(U) \mathcal{Y}(U)+\mathcal{D}^{\diamond_{0}}(U)+g(U)\right] \\
= & -\sum_{-; h} \mathcal{D}^{\diamond+}(U) \partial^{+}\left[\mathcal{D}^{\diamond_{0}}(U) \mathcal{Z}^{-}(U)\right] S^{+}\left[\mathcal{Z}^{+}(U) \mathcal{Y}(U)\right] \\
& -\sum_{-; h} \mathcal{D}^{\diamond+}(U) S^{+}\left[\mathcal{D}^{\diamond_{0}}(U) \mathcal{Z}^{-}(U)\right] \partial^{+}\left[\mathcal{Z}^{+}(U) \mathcal{Y}(U)\right] \\
& -\sum_{-; h} \mathcal{D}^{\diamond+}(U) \partial^{+}\left[\mathcal{D}^{\diamond \diamond_{0}}(U)+g(U)\right] .
\end{aligned}
$$

Using the definition (4.2) and the identities (4.30), we find

$$
\dot{x}=-\sum_{-; h} p(U) T^{+}\left[\mathcal{Z}^{-}(U)\right] \mathcal{D}^{\diamond_{0} ;+}(U) S^{+}\left[\mathcal{Z}^{+}(U) \mathcal{Y}(U)\right]
$$




$$
\begin{aligned}
& +\sum_{-; h} \mathcal{D}^{\diamond}(U) S^{+}\left[\mathcal{D}^{\diamond_{0}}(U) \mathcal{Z}^{-}(U)\right] p(U) \mathcal{Z}^{+}(U) \partial^{+}\left[\mathcal{D}^{\diamond_{0}}(U)+g(U)\right] \\
& -\sum_{-; h} \mathcal{D}^{\diamond+}(U) \partial^{+}\left[\mathcal{D}^{\diamond_{0}}(U)+g(U)\right] .
\end{aligned}
$$

Writing

$$
\mathcal{H}(U)=S^{+}\left[\mathcal{D}^{\diamond}(U) \mathcal{Z}^{-}(U)\right] p(U) \mathcal{Z}^{+}(U)-1,
$$

we see

$$
\begin{aligned}
\dot{x}= & -\sum_{-; h} p(U) T^{+}\left[\mathcal{Z}^{-}(U)\right] \mathcal{D}^{\diamond_{0} ;+}(U) S^{+}\left[\mathcal{Z}^{+}(U) \mathcal{Y}(U)\right] \\
& +\sum_{-; h} \mathcal{D}^{\diamond+}(U) \mathcal{H}(U) \partial^{+}\left[\mathcal{D}^{\diamond_{0}}(U)+g(U)\right] .
\end{aligned}
$$

Using (4.30) we now compute

$$
\begin{aligned}
\mathcal{H}(U) & =\left[S^{+}\left[\mathcal{D}^{\diamond_{0}}(U) \mathcal{Z}^{-}(U)\right] p(U)-\mathcal{Z}^{-}(U)\right] \mathcal{Z}^{+}(U) \\
& =\left[\mathcal{D}^{\diamond_{0}}(U) \mathcal{Z}^{-}(U) p(U)+\frac{1}{2} h \partial^{+}\left[\mathcal{D}^{\diamond_{0}}(U) \mathcal{Z}^{-}(U)\right] p(U)-\mathcal{Z}^{-}(U)\right] \mathcal{Z}^{+}(U) \\
& =\left[\mathcal{D}^{\diamond_{0}}(U) \mathcal{Z}^{-}(U) p(U)+\frac{1}{2} h \tilde{p}(U) T^{+}\left[\mathcal{Z}^{-}(U)\right] \mathcal{D}^{\diamond_{0} ;+}(U) p(U)-\mathcal{Z}^{-}(U)\right] \mathcal{Z}^{+}(U) .
\end{aligned}
$$

Exploiting (4.32) we obtain

$$
\mathcal{H}(U)=\left[-\widetilde{p}(U) \mathcal{Z}^{-}(U)+\frac{1}{2} h \widetilde{p}(U) T^{+}\left[\mathcal{Z}^{-}(U)\right] \mathcal{D}^{\diamond_{0} ;+}(U) p(U)\right] \mathcal{Z}^{+}(U),
$$

which using (4.26) yields

$$
\begin{aligned}
\mathcal{H}(U) & =\left[-\mathcal{Z}^{-}(U)-\frac{1}{2} h \partial^{+}\left[\mathcal{Z}^{-}(U)\right]\right] \tilde{p}(U) \mathcal{Z}^{+}(U) \\
& =-S^{+}\left[\mathcal{Z}^{-}(U)\right] \widetilde{p}(U) \mathcal{Z}^{+}(U) .
\end{aligned}
$$

In particular, recalling (4.8) we see

$$
\begin{aligned}
\dot{x}= & -\sum_{-; h} p(U) T^{+}\left[\mathcal{Z}^{-}(U)\right] \mathcal{D}^{\diamond_{0} ;+}(U) S^{+}\left[\mathcal{Z}^{+}(U) \mathcal{Y}(U)\right] \\
& -\sum_{-; h} S^{+}\left[\mathcal{Z}^{-}(U)\right] p(U) \mathcal{Z}^{+}(U) \partial^{+}\left[\mathcal{D}^{\diamond_{0}}(U)+g(U)\right] \\
= & \sum_{-; h} \partial^{+}[\mathcal{Y}(U)] .
\end{aligned}
$$

The desired conclusion $\dot{x}=\mathcal{Y}(U)$ now follows from the limit (4.9).

Lemma 4.10 Consider the setting of Proposition 4.2 and suppose that $(b)$ holds. Then we have $\dot{x}(t)=\mathcal{Y}(U(t))$ for all $0 \leq t \leq T$.

Proof Exploiting the identity (4.36), we compute

$$
\dot{x}=-\sum_{-; h} \mathcal{D}^{\diamond}(U) \partial^{+} \dot{U}
$$




$$
=-\sum_{-; h} \mathcal{D}^{\diamond}(U) \partial^{+}\left[\mathcal{D}^{\diamond_{0}}(U) \dot{x}+\mathcal{D}^{\diamond_{0}}(U)+g(U)\right] .
$$

Taking a difference, we obtain

$$
\partial^{+} \dot{x}+\mathcal{D}^{\diamond}(U) \partial^{+}\left[\mathcal{D}^{\diamond_{0}}(U) \dot{x}\right]=-\mathcal{D}^{\diamond}(U) \partial^{+}\left[\mathcal{D}^{\diamond \diamond_{0}}(U)+g(U)\right] .
$$

Using (4.26), we now observe that

$$
\begin{aligned}
\partial^{+}\left[\mathcal{Z}^{+}(U) \dot{x}\right]= & \partial^{+}\left[\mathcal{Z}^{+}(U)\right] T^{+} \dot{x}+\mathcal{Z}^{+}(U) \partial^{+}[\dot{x}] \\
= & \mathcal{Z}^{+}(U) p(U) \mathcal{D}^{\diamond_{0} ;+}(U) T^{+} \dot{x}+\mathcal{Z}^{+}(U) \partial^{+}[\dot{x}] \\
= & \mathcal{Z}^{+}(U) p(U)\left[\partial^{+}\left[\mathcal{D}^{\diamond_{0}}(U) \dot{x}\right]-\mathcal{D}^{\diamond_{0}}(U) \partial^{+} \dot{x}\right] \\
& +\mathcal{Z}^{+}(U) \partial^{+}[\dot{x}] \\
= & \mathcal{Z}^{+}(U)\left[\left(1-p(U) \mathcal{D}^{\diamond}(U)\right) \partial^{+}[\dot{x}]+p(U) \partial^{+}\left[\mathcal{D}^{\diamond}(U) \dot{x}\right]\right] .
\end{aligned}
$$

In particular, recalling (4.32) we see that

$$
\begin{aligned}
\partial^{+}\left[\mathcal{Z}^{+}(U) \dot{x}\right] & =\mathcal{Z}^{+}(U)\left[\tilde{p}(U) \partial^{+}[\dot{x}]+p(U) \partial^{+}\left[\mathcal{D}^{\diamond_{0}}(U) \dot{x}\right]\right] \\
& =\mathcal{Z}^{+}(U) \tilde{p}(U)\left[\partial^{+}[\dot{x}]+\mathcal{D}^{\diamond}(U) \partial^{+}\left[\mathcal{D}^{\diamond_{0}}(U) \dot{x}\right]\right] .
\end{aligned}
$$

Substituting (4.55), we find

$$
\partial^{+}\left[\mathcal{Z}^{+}(U) \dot{x}\right]=-\mathcal{Z}^{+}(U) p(U) \partial^{+}\left[\mathcal{D}^{\diamond \diamond 0}(U)+g(U)\right] .
$$

The limit (4.37) together with the inclusion $\mathcal{Z}^{+}(U) \in \ell^{\infty}$ implies that

$$
\lim _{j \rightarrow-\infty} \mathcal{Z}_{j h}^{+}(U) \dot{x}_{j h}=0 .
$$

In particular, we obtain

$$
\mathcal{Z}^{+}(U) \dot{x}=-\sum_{-; h} \mathcal{Z}^{+}(U) p(U) \partial^{+}\left[\mathcal{D}^{\diamond \diamond 0}(U)+g(U)\right],
$$

as desired.

Proof of Propostion 4.2 The result follows immediately from Lemma's 4.8-4.10.

Proof of Lemma 2.1 The statements follow directly from Propositions 3.1 and 4.1 .

Proof of Theorem 2.2 Suppose that (b) and (c) are satisfied. Writing

$$
y_{j h}(t)=x_{j h}(t)-j h
$$

we see that

$$
\lim _{j \rightarrow-\infty} y_{j h}(t)=0
$$

and

$$
\partial^{+} y(t)=\sqrt{1-\left[\partial^{+} U(t)\right]^{2}}-1 .
$$

This means that

$$
y(t)=\sum_{-; h} \partial^{+} y(t)=\sum_{-; h}\left[\sqrt{1-\left[\partial^{+} U(t)\right]^{2}}-1\right]
$$


and hence $x$ must satisfy (4.11). Together with (a) and (d), this allows us to apply Proposition 4.2 and conclude that $\dot{x}=\mathcal{Y}(U)$. Item (e) now directly implies that (2.25) holds.

Proof of Theorem 2.3 Items (a') and (b') together with (4.11) allow us to apply Proposition 4.2 and conclude that $\dot{x}=\mathcal{Y}(U)$. Together with (c') this implies that (2.2) holds. Item (a) follows from Lemma 4.8, (b) and (d) are immediate and finally (c) follows from the fact that $r_{U}^{+}-1 \in \ell^{1}(h \mathbb{Z} ; \mathbb{R})$.

\section{Approximate Products}

In the remainder of this paper we set out to establish Proposition 2.4. In principle, it suffices to obtain Lipschitz bounds on the right-hand-side of (2.25). However, in preparation for the sequel paper [32] we obtain far more detailed information here in the upcoming sections. In particular, we derive an approximate expression for the linear terms and establish delicate bounds for the nonlinear residual that require as few discrete derivatives as possible.

The key issue is that convoluted expressions such as (2.17) must be kept under control. We will achieve this by two successive rounds of approximations, in which we substitute simplified expressions for the individual factors appearing in (2.25). The first round is performed in this paper, the second round is reserved for [32]. This will allow us to extract a tractable expression for the linearization of (2.25) around the (stretched) continuum travelling wave, which we analyze in [31].

In this section we introduce the basic framework that we use for these two simplification rounds. In particular, we focus on two types of products that appear in our main equation (2.25). We will introduce several bookkeeping tools that allow us to track how errors in the individual factors propagate through the product structure.

We emphasize that the individual core estimates underlying our bounds are rather basic. However, these estimates need to be applied a large number of times, all in a slightly different setting that sometimes requires the same factor to be estimated in different norms. We feel that writing this out in full would be prohibitively voluminous without leading to any substantial insight. We therefore take an alternative approach and formulate a number of structural conditions on the products (see $\$ 5.1$ ) and individual factors (see $\$ 5.2$ ) that allow for a unified result (see §5.3).

\subsection{Product Structure}

For any integer $k \geq 1$ and any sequence

$$
\mathbf{q}=\left(q_{1}, q_{2}, \ldots, q_{k}\right) \in\{2, \infty\}^{k},
$$

we introduce the notation

$$
\ell_{h}^{\mathbf{q}}=\ell_{h}^{q_{1}} \times \ell_{h}^{q_{2}} \times \cdots \times \ell_{h}^{q_{k}} .
$$

Writing

$$
\mathbf{q}_{\pi}=\left(q_{\pi ; 1}, q_{\pi ; 2}, \ldots, q_{\pi ; k}\right) \in\{2, \infty\}^{k},
$$

we are interested in maps

$$
\pi: \ell_{h}^{\mathbf{q}_{\pi}} \rightarrow \ell_{h}^{2}
$$


that are bounded and multi-linear in the following sense.

$(\mathrm{h} \pi)$ Consider any set

$$
\mathbf{v}=\left(v_{1}, \ldots, v_{k}\right) \in \ell_{h}^{\mathbf{q}_{\pi}}
$$

Then we have the estimate

$$
\|\pi[\mathbf{v}]\|_{\ell_{h}^{2}} \leq K\left\|v_{1}\right\|_{\ell_{h}^{q_{\pi ; 1}}} \times \cdots \times\left\|v_{k}\right\|_{\ell_{h}^{q_{\pi ; k}}}
$$

for some constant $K>0$ that does not depend on $\mathbf{v}$ and $h>0$. In addition, if there is an integer $1 \leq i \leq k$ for which the decomposition

$$
v_{i}=\lambda_{A} v_{i}^{A}+\lambda_{B} v_{i}^{B}
$$

holds, with $v_{i}^{\#} \in \ell_{h}^{q_{\pi ; i}}$ and $\lambda_{\#} \in \mathbb{R}$ for $\# \in\{A, B\}$, then we have

$$
\pi[\mathbf{v}]=\lambda_{A} \pi\left[v_{1}, \ldots, v_{i}^{A}, \ldots, v_{k}\right]+\lambda_{B} \pi\left[v_{1}, \ldots, v_{i}^{B}, \ldots, v_{k}\right] .
$$

We say that any sequence (5.1) is admissable for $\pi$ if there is a constant $K>1$ so that the bound

$$
\|\pi[v]\|_{\ell_{h}^{2}} \leq K\left\|v_{1}\right\|_{\ell_{h}^{q_{1}}} \times \cdots \times\left\|v_{k}\right\|_{\ell_{h}^{q_{k}}}
$$

holds for any

$$
v \in \ell_{h}^{\mathbf{q}_{\pi}} \cap \ell_{h}^{\mathbf{q}}
$$

and any $h>0$.

Our first two results describe the two types of products that will appear during our analysis of (2.25). In both cases it is straightforward to verify that our condition (h $\pi$ ) holds.

Lemma 5.1 Pick $k \geq 1$. Assume that

$$
\mathbf{q}_{\pi}=\left(q_{\pi ; 1}, \ldots, q_{\pi ; k}\right) \in\{2, \infty\}^{k}
$$

is a sequence containing precisely one 2 and suppose that the map

$$
\pi: \ell^{\mathbf{q}_{\pi}} \rightarrow \ell_{h}^{2}
$$

is given by

$$
\pi\left[v_{1}, \ldots, v_{k}\right]=v_{1} v_{2} \cdots v_{k} .
$$

Then the pair $\left(\mathbf{q}_{\pi}, \pi\right)$ satisfies condition $(h \pi)$.

Proof This follows directly from the bound

$$
\left\|v_{1} \cdots v_{k}\right\|_{\ell_{h}^{2}} \leq\left\|v_{1}\right\|_{\ell_{h}^{2}}\left\|v_{2}\right\|_{\ell_{h}^{\infty}} \cdots\left\|v_{k}\right\|_{\ell_{h}^{\infty}}
$$

and rearrangements thereof.

Lemma 5.2 Pick $k_{1} \geq 1$ and $k_{2} \geq 2$ and write $k=k_{1}+k_{2}$. Assume that

$$
\mathbf{q}_{\pi}=\left(q_{\pi ; 1}, \ldots, q_{\pi ; k}\right) \in\{2, \infty\}^{k}
$$

is a sequence containing precisely one 2 in the first $k_{1}$ positions and precisely two 2 's in the last $k_{2}$ positions. Recalling the notation (3.9), assume furthermore that the map

$$
\pi: \ell_{h}^{\mathbf{q}_{\pi}} \rightarrow \ell_{h}^{2}
$$


is given by

$$
\pi\left[v_{1}, \ldots, v_{k}\right]=v_{1} \cdots v_{k_{1}} \sum_{-; h} v_{k_{1}+1} \cdots v_{k} .
$$

Then the pair $\left(\mathbf{q}_{\pi}, \pi\right)$ satisfies condition $(h \pi)$.

Proof This follows directly from the bound

$$
\begin{aligned}
& \left\|\pi\left[v_{1}, \ldots, v_{k}\right]\right\|_{\ell_{h}^{2}} \\
& \leq\left\|v_{1}\right\|_{\ell_{h}^{2}}\left\|v_{2}\right\|_{\ell_{h}^{\infty}} \cdots\left\|v_{k_{1}}\right\|_{\ell_{h}^{\infty}}\left\|v_{k_{1}+1}\right\|_{\ell_{h}^{2}}\left\|v_{k_{1}+2}\right\|_{\ell_{h}^{2}}\left\|v_{k_{1}+3}\right\|_{\ell_{h}^{\infty}} \cdots\left\|v_{k}\right\|_{\ell_{h}^{\infty}}
\end{aligned}
$$

and rearrangements thereof.

Our final observation allows us to construct admissable sequences for the multi-linear maps (5.13) and (5.17) by simply swapping suitable exponents. This freedom to be able to use a $\ell_{h}^{2}$-norm on each of the factors will turn out to be crucial to complete the construction of the travelling waves in [32]. Indeed, as a general rule of thumb, we want to avoid references to the supremum norm of second differences.

Lemma 5.3 Consider the setting of either Lemma 5.1 or Lemma 5.2. Pick any integer $1 \leq$ $i_{*} \leq k$ for which $q_{\pi ; i_{*}}=\infty$. Then there is an integer

$$
1 \leq j_{*}\left[i_{*}\right] \leq k
$$

that has

$$
q_{\pi ; j_{*}\left[i_{*}\right]}=2
$$

and for which the swapped sequence

$$
\mathbf{q}_{i_{*}}=\left(q_{i_{*} ; 1}, \ldots, q_{i_{*} ; k}\right)
$$

defined by

$$
q_{i_{*} ; j}= \begin{cases}q_{\pi ; j} & \text { if } j \notin\left\{i_{*}, j_{*}\right\} \\ 2 & \text { if } j=i_{*}, \\ \infty & \text { if } j=j_{*},\end{cases}
$$

is admissable for $\pi$.

Proof This follows directly by inspecting (5.14) and (5.18).

\subsection{Approximate Nonlinearities}

Fixing a multi-linear map $\pi$ as discussed above, our goal is to study nonlinear functions of the form

$$
\Omega_{h ; \kappa} \ni U \mapsto \pi\left[f_{1}(U), \ldots, f_{k}(U)\right] .
$$

We take the point of view that each nonlinearity $f_{i}$ together with its derivative comes paired with an approximant $f_{i}$; apx respectively $f_{i}$;in. These two approximants should be thought of as simplified versions of $f_{i}$ and $D f_{i}$ that are much easier to handle in computations, while still accurate to some desired order in $h$.

For now however, we simply impose the following condition on each of the nonlinearities in (5.23). We remark that the set $Q_{f}$ should be thought of as the set of exponents $q \in\{2, \infty\}$ for which $f$ maps into $\ell_{h}^{q}$. 
(hf) We have $Q_{f} \subset\{2, \infty\}$. For any $U \in \Omega_{h ; \kappa}$ and $q \in Q_{f}$ we have the inclusions

$$
f(U) \in \ell_{h}^{q}, \quad f_{\text {apx }}(U) \in \ell_{h}^{q}, \quad f_{\text {lin; } U} \in \mathcal{L}\left(\ell_{h}^{2} ; \ell_{h}^{q}\right) .
$$

In addition, for each $q \in Q_{f}$ there exists a constant $K_{q}>0$ and a semi-norm [- $]_{f ; q, h}$ on $\ell_{h}^{2}$ so that the following properties are true.

(a) The inequality

$$
\|f(U)\|_{\ell_{h}^{q}}+\left\|f_{\text {apx }}(U)\right\|_{\ell_{h}^{q}} \leq K_{q}
$$

holds for all $h>0$ and $U \in \Omega_{h ; \kappa}$.

(b) The inequality

$$
[V]_{f ; q, h} \leq K_{q}
$$

holds for all $h>0$ and $V \in \mathcal{V}_{h ; \kappa}$.

(c) The Lipschitz estimate

$$
\left\|f\left(U^{(1)}\right)-f\left(U^{(2)}\right)\right\|_{\ell_{h}^{q}} \leq K_{q}\left[U^{(1)}-U^{(2)}\right]_{f ; q, h}
$$

holds for all $h>0$ and all pairs $\left(U^{(1)}, U^{(2)}\right) \in \Omega_{h ; \kappa}^{2}$.

(d) For every $h>0$, the inequality

$$
\left\|f_{\operatorname{lin} ; U}[V]\right\|_{\ell_{h}^{q}} \leq K_{q}[V]_{f ; q, h}
$$

holds for all $U \in \Omega_{h ; \kappa}$ and $V \in \ell_{h}^{2}$.

To obtain sharp estimates it is sometimes necessary to decompose the approximate linearization $f_{\text {lin }}$ into two parts. Both parts can be evaluated in their own preferred norms, which do not necessarily have to be an element of the set $Q_{f}$ discussed in condition (h $f$ ) above.

$(h f)_{\text {lin }}$ We have $Q_{f ; \text { lin }}^{A} \subset\{2, \infty\}$ and $Q_{f ; \text { lin }}^{B} \subset\{2, \infty\}$. For all $h>0, U \in \Omega_{h ; \kappa}$ and $V \in \ell_{h}^{2}$, we can make the decomposition

$$
f_{\operatorname{lin} ; U}[V]=f_{\operatorname{lin} ; U}^{A}[V]+f_{\operatorname{lin} ; U}^{B}[V],
$$

in which $f_{\operatorname{lin} ; U}^{A}[V] \in \ell_{h}^{q}$ for every $q \in Q_{f ; \operatorname{lin}}^{A}$ and $f_{\operatorname{lin} ; U}^{B}[V] \in \ell_{h}^{q}$ for every $q \in Q_{f ; \operatorname{lin}}^{B}$.

Our final condition concerns the residual term

$$
f_{\mathrm{nl} ; U}(V)=f(U+V)-f(U)-f_{\operatorname{lin} ; U}[V],
$$

which at times also needs to be decomposed into two parts that require separate norms.

$(h f)_{\mathrm{nl}}$ We have $Q_{f ; \mathrm{nl}}^{A} \subset\{2, \infty\}$ and $Q_{f ; \mathrm{nl}}^{B} \subset\{2, \infty\}$. For all $h>0, U \in \Omega_{h ; \kappa}$ and $V \in \ell_{h}^{2}$ for which $U+V \in \Omega_{h ; \kappa}$, we can make the decomposition

$$
f_{\mathrm{nl} ; U}(V)=f_{\mathrm{nl} ; U}^{A}(V)+f_{\mathrm{nl} ; U}^{B}(V),
$$

in which $f_{\mathrm{nl} ; U}^{A}(V) \in \ell_{h}^{q}$ for every $q \in Q_{f ; \mathrm{nl}}^{A}$ and $f_{\mathrm{nl} ; U}^{B}(V) \in \ell_{h}^{q}$ for every $q \in Q_{f ; \mathrm{nl}}^{B}$. 


\subsection{Approximation Errors}

We are now ready to formulate and establish our approximation result. To set the stage, let us consider the simple example

$$
P(U)=f_{1}(U) f_{2}(U), \quad Q_{f_{1}}=\{2, \infty\}, \quad Q_{f_{2}}=\{\infty\},
$$

which leads naturally to the approximations

$$
P_{\text {apx }}(U)=f_{1 ; \text { apx }}(U) f_{2 ; \text { apx }}(U), \quad P_{\operatorname{lin} ; U}[V]=f_{1 ; \operatorname{lin} ; U}[V] f_{2 ; \text { apx }}(U)+f_{1 ; \text { apx }}(U) f_{2 ; \operatorname{lin} ; U}[V] .
$$

Our main result below shows that the individual approximation errors $f_{i}(U)-f_{i \text {; apx }}(U)$ and $f_{i ; \mathrm{nl} ; U}$ propagate through to the product $P$ in a relatively clean fashion, using combinations of the bounds introduced in $\$ 5.2$. For example, in our case here we can estimate

$$
\begin{aligned}
\left\|P(U)-P_{\mathrm{apx}}(U)\right\|_{\ell_{h}^{2}} \leq & \left\|f_{1}(U)-f_{1 ; \mathrm{apx}}(U)\right\|_{\ell_{h}^{2}}\left\|f_{2}(U)\right\|_{\ell_{h}^{\infty}} \\
& +\left\|f_{1 ; \mathrm{apx}}(U)\right\|_{\ell_{h}^{2}}\left\|f_{2}(U)-f_{2 ; \text { apx }}(U)\right\|_{\ell_{h}^{\infty}},
\end{aligned}
$$

but such direct bounds quickly become unwieldy when the number of factors increases, especially for the nonlinear residual (5.45). This becomes even more troublesome when certain factors come with restrictions on the available norms.

Indeed, the main feature of the result below is that only needs to check whether the available exponent sets match together to respect the product structure of $\pi$. Returning to our example (5.32), one cannot include $\left\|f_{1 ; \mathrm{nl} ; U}[V]\right\|_{\ell_{h}^{\infty}}$ in the term $\mathcal{J}_{\mathrm{nl} ; U}$ appearing in the estimate (5.47), because this would require the second nonlinearity to be measured with respect to the $\ell_{h}^{2}$ norm, which is not included in the exponent set $Q_{f_{2}}$. All in all, we use this result to transform potentially lengthy product estimates into bookkeeping questions, which can be resolved by carefully defining suitable exponent sets. The discussion in $\$ 7.2$ should be seen in this light.

Proposition 5.4 Fix $k \geq 1$ and $0<\kappa<\frac{1}{12}$, consider the pair $\left(\mathbf{q}_{\pi}, \pi\right)$ defined in (5.3)-(5.4) and assume that condition $(h \pi)$ holds. In addition, consider a set

$$
\left\{f_{i}, f_{i ; \mathrm{apx}}, f_{i ; \text { lin }}, Q_{f_{i}}, Q_{f_{i} ; \text { lin }}^{A}, Q_{f_{i} ; \mathrm{nl}}^{A}, Q_{f_{i} ; \text { lin }}^{B}, Q_{f_{i} ; \mathrm{nl}}^{B}\right\}_{i=1}^{k}
$$

of nonlinearities with their associated approximants and exponents that satisfy the following properties.

(a) For every $1 \leq i \leq k$, the set $\left\{f_{i}, f_{i ; \mathrm{apx}}, f_{i ; \mathrm{nl}}, Q_{f_{i}}\right\}$ satisfies condition $(h f)$ and the set

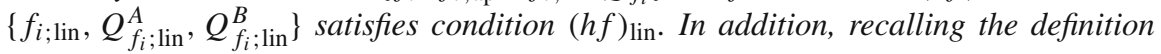
(5.30), the set $\left\{f_{i ; \mathrm{nl}}, Q_{f_{i} ; \mathrm{nl}}^{A}, Q_{f_{i} ; \mathrm{nl}}^{B}\right\}$ satisfies condition $(h f)_{\mathrm{nl}}$.

(b) We have $q_{\pi ; i} \in Q_{f_{i}}$ for $1 \leq i \leq k$.

(c) For every $1 \leq i \leq k$, there are $\sigma_{i ; \mathrm{nl}}^{A} \in Q_{f_{i} ; \mathrm{nl}}^{A}$ and $\sigma_{i ; \mathrm{nl}}^{B} \in Q_{f_{i} ; \mathrm{nl}}^{B}$ together with sets

$$
q_{i, \mathrm{nl}}^{A}=\left(q_{i, \mathrm{nl} ; 1}^{A}, \ldots, q_{i, \mathrm{nl} ; k}^{A}\right), \quad q_{i, \mathrm{nl}}^{B}=\left(q_{i, \mathrm{nl} ; 1}^{B}, \ldots, q_{i, \mathrm{nl} ; k}^{B}\right)
$$

that are admissible for $\pi$, which have

$$
q_{i, \mathrm{nl} ; i}^{A}=\sigma_{i ; \mathrm{nl}}^{A}, \quad q_{i, \mathrm{nl} ; i}^{B}=\sigma_{i ; \mathrm{nl}}^{B}
$$

and

$$
q_{i, \mathrm{nl} ; j}^{A} \in Q_{f_{j}}, \quad q_{i, \mathrm{nl} ; j}^{B} \in Q_{f_{j}}
$$


for all $j \neq i$.

(d) For every pair $(i, j) \in\{1, \ldots, k\}^{2}$ with $i \neq j$, there are

$$
\sigma_{i j ; \text { lin }}^{A} \in Q_{f_{i} ; \text { lin }}^{A}, \quad \tau_{i j ; \operatorname{lin}}^{A} \in Q_{f_{j}}, \quad \sigma_{i j ; \operatorname{lin}}^{B} \in Q_{f_{i} ; \operatorname{lin}}^{B}, \quad \tau_{i j ; \operatorname{lin}}^{B} \in Q_{f_{j}},
$$

together with two sets

$$
q_{i j, \operatorname{lin}}^{A}=\left(q_{i j, \operatorname{lin} ; 1}^{A}, \ldots, q_{i j, \operatorname{lin} ; k}^{A}\right), \quad q_{i j, \operatorname{lin}}^{B}=\left(q_{i j, \operatorname{lin} ; 1}^{B}, \ldots, q_{i j, \operatorname{lin} ; k}^{B}\right)
$$

that are admissible for $\pi$, which have

$$
q_{i j, \operatorname{lin} ; i}^{A}=\sigma_{i j ; \operatorname{lin}}^{A}, \quad q_{i j, \operatorname{lin} ; j}^{A}=\tau_{i j ; \operatorname{lin}}^{A} \quad q_{i j, \operatorname{lin} ; i}^{B}=\sigma_{i j ; \operatorname{lin}}^{B}, \quad q_{i j, \operatorname{lin} ; j}^{B}=\tau_{i j ; \operatorname{lin}}^{B}
$$

and

$$
q_{i j, \operatorname{lin} ; k^{\prime}}^{A} \in Q_{f_{k}^{\prime}}, \quad q_{i j, \operatorname{lin} ; k^{\prime}}^{B} \in Q_{f_{k}^{\prime}}
$$

for all $k^{\prime} \notin\{i, j\}$.

Consider the map

$$
P: \Omega_{h ; \kappa} \rightarrow \ell_{h}^{2}, \quad U \mapsto \pi\left[f_{1}(U), \ldots, f_{k}(U)\right] .
$$

For any $U \in \Omega_{h ; \kappa}$ and $V \in \ell_{h}^{2}$, write

$$
\begin{aligned}
P_{\mathrm{apx}}(U)= & \pi\left[f_{1 ; \mathrm{apx}}(U), \ldots, f_{k ; \mathrm{apx}}(U)\right] \\
P_{\operatorname{lin} ; U}[V]= & \pi\left[f_{1 ; \operatorname{lin} ; U}[V], f_{2 ; \operatorname{apx}}(U), \ldots, f_{k ; \text { apx }}(U)\right] \\
& +\pi\left[f_{1 ; \mathrm{apx}}(U), f_{2 ; \operatorname{lin} ; U}[V], \ldots, f_{k ; \mathrm{apx}}(U)\right] \\
& +\cdots+\pi\left[f_{1 ; \mathrm{apx}}(U), \ldots, f_{k-1 ; \operatorname{apx}}(U), f_{k ; \operatorname{lin} ; U}[V]\right] .
\end{aligned}
$$

In addition, for any $U \in \Omega_{h ; \kappa}$ and $V \in \ell_{h}^{2}$ for which $U+V \in \Omega_{h ; \kappa}$, write

$$
P_{\mathrm{nl} ; U}(V)=P(U+V)-P(U)-P_{\operatorname{lin} ; U}[V] .
$$

Then there exists a constant $K>0$ so that for any $h>0$ and $U \in \Omega_{h ; \kappa}$ the bound

$$
\left\|P(U)-P_{\text {apx }}(U)\right\|_{\ell_{h}^{2}} \leq K \sum_{i=1}^{k}\left\|f_{i}(U)-f_{i ; \mathrm{apx}}(U)\right\|_{q_{\pi ; i}}
$$

holds, while for any $h>0, U \in \Omega_{h ; \kappa}$ and $V \in \ell_{h}^{2}$ for which $U+V \in \Omega_{h ; \kappa}$ we have the estimate

$$
\left\|P_{\mathrm{nl} ; U}(V)\right\|_{\ell_{h}^{2}} \leq K \mathcal{J}_{\mathrm{nl} ; U}(V)+K \mathcal{J}_{\text {cross } ; U}(V)+K \mathcal{J}_{\mathrm{apx} ; U}(V) .
$$

Here we have introduced the expressions

$$
\mathcal{J}_{\mathrm{nl} ; U}(V)=\sum_{i=1}^{k}\left[\left\|f_{i ; \mathrm{nl}}^{A}(V)\right\|_{\sigma_{i ; \mathrm{nl}}^{A}}+\left\|f_{i ; \mathrm{nl}}^{B}(V)\right\|_{\sigma_{i ; \mathrm{nl}}^{B}}\right],
$$

together with

$$
\mathcal{J}_{\text {cross } ; U}(V)=\sum_{i=1}^{k} \sum_{j \neq i}\left\|f_{i ; \operatorname{lin}}^{A}[V]\right\|_{\sigma_{i j ; \operatorname{lin}}^{A}}[V]_{f_{j} ; \tau_{i j ; \operatorname{lin}}^{A}, h}
$$




$$
+\sum_{i=1}^{k} \sum_{j \neq i}\left\|f_{i ; \operatorname{lin}}^{B}[V]\right\|_{\sigma_{i j ; \operatorname{lin}}^{B}}[V]_{f_{j} ; \tau_{i j ; \operatorname{lin}}^{B}, h}
$$

and finally

$$
\begin{aligned}
\mathcal{J}_{\text {apx } ; U}(V)= & \sum_{i=1}^{k} \sum_{j \neq i}\left\|f_{i ; \operatorname{lin}}^{A}[V]\right\|_{\sigma_{i j ; \operatorname{lin}}^{A}}\left\|f_{j}(U)-f_{j ; \text { apx }}(U)\right\|_{\tau_{i j ; \operatorname{lin}}^{A}} \\
& +\sum_{i=1}^{k} \sum_{j \neq i}\left\|f_{i ; \operatorname{lin}}^{B}[V]\right\|_{\sigma_{i j ; \operatorname{lin}}^{B}}\left\|f_{j}(U)-f_{j ; \text { apx }}(U)\right\|_{\tau_{i j ; \operatorname{lin}}^{B}} .
\end{aligned}
$$

Proof Pick $1 \leq i \leq k$, any $h>0, U \in \Omega_{h ; \kappa}$ and $V \in \ell_{h}^{2}$ for which $U+V \in \Omega_{h ; \kappa}$. We remark that all the primed constants below are independent of these specific choices.

By definition, the condition on $V$ means that

$$
V=V^{(a)}-V^{(b)}
$$

with $V^{(\#)} \in \mathcal{V}_{h ; \kappa}$ for $\# \in\{a, b\}$. Exploiting (5.26), this shows that we have the uniform bound

$$
[V]_{f_{i} ; q, h} \leq C_{1}^{\prime}
$$

for any $q \in Q_{f_{i}}$. In addition, we may use (5.27) and (5.28) to obtain the rough estimate

$$
\begin{aligned}
\left\|f_{i ; \mathrm{nl}}(V)\right\|_{\ell_{h}^{q}} & \leq\left\|f_{i}(U+V)-f_{i}(U)\right\|_{q}+\left\|f_{i ; \operatorname{lin}}[V]\right\|_{\ell_{h}^{q}} \\
& \leq C_{2}^{\prime}[V]_{i ; q, h},
\end{aligned}
$$

which gives

$$
\left\|f_{i ; \operatorname{lin} ; U}[V]\right\|_{\ell_{h}^{q}}+\left\|f_{i ; \mathrm{nl} ; U}(V)\right\|_{\ell_{h}^{q}} \leq C_{3}^{\prime}[V]_{i ; q, h} .
$$

In addition, using (5.25) and (5.52), we obtain the uniform bound

$$
\left\|f_{i}(U)\right\|_{\ell_{h}^{q}}+\left\|f_{i ; \operatorname{lin} ; U}[V]\right\|_{\ell_{h}^{q}}+\left\|f_{i ; \mathrm{nl} ; U}(V)\right\|_{\ell_{h}^{q}} \leq C_{4}^{\prime}+C_{3}^{\prime}[V]_{i ; q, h} \leq C_{5}^{\prime} .
$$

Observe that

$$
\begin{aligned}
& P(U+V)-P(U)=\pi\left[f_{1}(U+V), \ldots, f_{k}(U+V)\right]-\pi\left[f_{1}(U), \ldots, f_{k}(U)\right] \\
& =\pi\left[f_{1}(U)+f_{1 ; \operatorname{lin} ; U}[V]+f_{1 ; \mathrm{nl} ; U}(V), \ldots, f_{k}(U)+f_{k ; \operatorname{lin} ; U}[V]+f_{k ; \mathrm{nl} ; U}(V)\right] \\
& -\pi\left[f_{1}(U), \ldots, f_{k}(U)\right] \text {. }
\end{aligned}
$$

In particular, writing

$$
\begin{aligned}
P_{\operatorname{lin} ; U ; I}[V]= & \pi\left[f_{1 ; \operatorname{lin} ; U}[V], f_{2}(U), \ldots, f_{k}(U)\right] \\
& +\pi\left[f_{1}(U), f_{2 ; \operatorname{lin} ; U}[V], \ldots, f_{k}(U)\right] \\
& +\cdots+\pi\left[f_{1}(U), \ldots, f_{k-1}(U), f_{k ; \operatorname{lin} ; U}[V]\right],
\end{aligned}
$$

together with

$$
P_{\mathrm{nl} ; U ; I}(V)=P(U+V)-P(U)-P_{\operatorname{lin} ; U ; I}[V],
$$

the bounds (5.54) and (5.55) allow us to expand out $P_{\mathrm{nl} ; U ; I}[V]$ and obtain

$$
\left\|P_{\mathrm{nl} ; U ; I}[V]\right\|_{\ell_{h}^{2}} \leq C_{6}^{\prime} \mathcal{J}_{\mathrm{nl} ; U}(V)+C_{6}^{\prime} \mathcal{J}_{\text {cross } ; U}(V) .
$$




\section{Upon writing}

$$
\mathcal{J}_{1}=\pi\left[f_{1 ; \operatorname{lin} ; U}[V], f_{2}(U), \ldots, f_{k}(U)\right]-\pi\left[f_{1 ; \operatorname{lin} ; U}[V], f_{2 ; \text { apx }}(U), \ldots, f_{k ; \text { apx }}(U)\right],
$$

we see by multi-linearity that

$$
\begin{aligned}
\mathcal{J}_{1}= & \pi\left[f_{1 ; \operatorname{lin} ; U}[V], f_{2}(U)-f_{2 ; \text { apx }}(U), \ldots, f_{k}(U)\right] \\
& +\pi\left[f_{1 ; \operatorname{lin} ; U}[V], f_{2 ; \text { apx }}(U), f_{3}(U)-f_{3 ; \text { apx }}(U), \ldots, f_{k}(U)\right] \\
& +\cdots+\pi\left[f_{1 ; \operatorname{lin} ; U}[V], f_{2 ; \text { apx }}(U), \ldots, f_{k-1 ; \operatorname{apx}}(U), f_{k}(U)-f_{k ; \text { apx }}(U)\right] .
\end{aligned}
$$

In particular, exploiting (5.25), we obtain the bound

$$
\left\|\mathcal{J}_{1}\right\|_{\ell_{h}^{2}} \leq C_{7}^{\prime} \mathcal{J}_{\text {apx } ; U}(V) .
$$

Repeating this computation for the remaining indices shows that also

$$
\left\|P_{\operatorname{lin} ; U}[V]-P_{\operatorname{lin} ; U ; I}[V]\right\|_{\ell_{h}^{2}} \leq C_{8}^{\prime} \mathcal{J}_{\text {apx } ; U}(V),
$$

which establishes (5.47). The estimate (5.46) can be obtained in a similar, but much easier fashion.

\section{The Full Nonlinearity}

In this section we study the function

$$
\mathcal{G}(U)=\mathcal{D}^{\diamond_{0}}(U) \mathcal{Y}(U)+\mathcal{D}^{\diamond_{0}}(U)+g(U),
$$

which contains all the terms on the right-hand side of our main reduced equation (2.25). In addition, we study the discrete derivative

$$
\mathcal{G}^{+}(U)=\partial^{+}[\mathcal{G}(U)]
$$

In principle the results in $\S 3$ and $\S 4$ provide explicit expressions for all these terms, but the main issue here is that the expression (4.8) features a third order derivative that cannot be controlled uniformly for $U \in \Omega_{h ; \kappa}$ and $h>0$. This is particularly dangerous for many types of bifurcation arguments, including the one that we develop in the sequel paper [32]. Indeed, we can only expect our unperturbed problem to generate two derivatives, in line with the continuous theory discussed in $\S 1$.

This can be repaired by a discrete summation-by-parts procedure that we carry out in this section. Naturally, the term $\mathcal{G}^{+}(U)$ will feature third derivatives, but as a consequence of the discrete differentiation the relevant linear operator also generates an extra derivative.

In order to state our results, we need to introduce the three auxiliary functions

$$
\begin{aligned}
& p_{A}^{\diamond_{+}}(U)=\frac{S^{+}\left[1+\mathcal{D}^{\diamond+}(U) \mathcal{D}^{\diamond_{0}}(U)\right]}{P^{+}\left[1+\mathcal{D}^{\diamond}(U) \mathcal{D}^{\diamond_{0}}(U)\right]}, \\
& p_{B}^{\diamond_{+}}(U)=-\frac{S^{+}\left[\mathcal{D}^{\diamond}(U)\right] S^{+}\left[\mathcal{D}^{\diamond_{0}}(U)\right]}{P^{+}\left[1+\mathcal{D}^{\diamond}(U) \mathcal{D}^{\diamond_{0}}(U)\right]}, \\
& p^{\diamond_{0}}(U)=-\frac{S^{+}\left[\mathcal{D}^{\diamond}(U)\right] S^{+}\left[\mathcal{D}^{\diamond}(U)\right]}{P^{+}\left[1+\mathcal{D}^{\diamond}(U) \mathcal{D}^{\diamond_{0}}(U)\right]},
\end{aligned}
$$


together with the convenient shorthand

$$
p^{\diamond+}(U)=p_{A}^{\diamond+}(U)+p_{B}^{\diamond+}(U) .
$$

Our first main result here shows how these functions can be used to describe $\partial^{+} p(U)$; see (4.2).

Proposition 6.1 Fix $0<\kappa<\frac{1}{12}$ and $h>0$. Then for any $U \in \Omega_{h ; \kappa}$ we have the inclusions

$$
p_{A}^{\diamond^{+}}(U) \in \ell^{\infty}(h \mathbb{Z} ; \mathbb{R}), \quad p_{B}^{\diamond_{+}^{+}}(U) \in \ell^{2}(h \mathbb{Z} ; \mathbb{R}), \quad p^{\diamond_{0}}(U) \in \ell^{2}(h \mathbb{Z} ; \mathbb{R}) .
$$

In addition, we have the identity

$$
\partial^{+} p(U)=p^{\diamond+}(U) \mathcal{D}^{\diamond+;+}(U)+p^{\diamond}(U) \mathcal{D}^{\diamond_{0} ;+}(U) .
$$

We now have all the necessary ingredients to define the functions

$$
\begin{aligned}
& \mathcal{Y}_{1}(U)=\mathcal{D}^{\diamond_{0}}(U) \mathcal{Z}^{-}(U), \\
& \mathcal{Y}_{2}(U)=\mathcal{D}^{\diamond \diamond_{0}}(U)+g(U),
\end{aligned}
$$

together with

$$
\begin{aligned}
& \mathcal{X}_{A}(U)=p(U) \mathcal{Z}^{+}(U), \\
& \mathcal{X}_{B}(U)=S^{+}\left[\mathcal{Z}^{+}(U)\right] p^{\diamond+}(U), \\
& \mathcal{X}_{C}(U)=S^{+}\left[\mathcal{Z}^{+}(U)\right] p^{\diamond_{0}}(U), \\
& \mathcal{X}_{D}(U)=S^{+}[p(U)] \mathcal{Z}^{+}(U) p(U) .
\end{aligned}
$$

We note here that $\mathcal{Y}_{2}$ contains the most important terms and will therefore receive the most attention. The $\mathcal{X}$ expressions play an auxiliary role, allowing for a relatively streamlined decomposition of our nonlinearity. Indeed, our second main result shows that $\mathcal{G}(U)$ can be split into the four components

$$
\begin{aligned}
& \mathcal{G}_{A}(U)=\left[1-\mathcal{Y}_{1}(U) T^{-}\left[\mathcal{X}_{A}(U)\right]\right] \mathcal{Y}_{2}(U), \\
& \mathcal{G}_{B}(U)=\mathcal{Y}_{1}(U) \sum_{-; h} \mathcal{Y}_{2}(U) T^{-}\left[\mathcal{X}_{B}(U) \mathcal{D}^{\diamond_{+} ;+}(U)\right], \\
& \mathcal{G}_{C}(U)=\mathcal{Y}_{1}(U) \sum_{-; h} \mathcal{Y}_{2}(U) T^{-}\left[\mathcal{X}_{C}(U) \mathcal{D}^{\diamond_{0} ;+}(U)\right], \\
& \mathcal{G}_{D}(U)=\mathcal{Y}_{1}(U) \sum_{-; h} \mathcal{Y}_{2}(U) T^{-}\left[\mathcal{X}_{D}(U) \mathcal{D}^{\diamond_{0} ;+}(U)\right] .
\end{aligned}
$$

Proposition 6.2 Suppose that $(\mathrm{Hg})$ is satisfied and fix $0<\kappa<\frac{1}{12}$ and $h>0$. Then for any $U \in \Omega_{h ; \kappa}$ we have the identity

$$
\mathcal{G}(U)=\mathcal{G}_{A}(U)+\mathcal{G}_{B}(U)+\mathcal{G}_{C}(U)+\mathcal{G}_{D}(U) .
$$

Turning to $\mathcal{G}^{+}(U)$, we introduce the functions

$$
\mathcal{Y}_{1}^{+}(U)=\partial^{+}\left[\mathcal{Y}_{1}(U)\right], \quad \mathcal{Y}_{2}^{+}(U)=\partial^{+}\left[\mathcal{Y}_{2}(U)\right] .
$$

Recalling (4.2) and using (4.30), one obtains the identities

$$
\mathcal{Y}_{1}^{+}(U)=\tilde{p}(U) \mathcal{D}^{\diamond ;+}(U) T^{+}\left[\mathcal{Z}^{-}(U)\right],
$$




$$
\mathcal{Y}_{2}^{+}(U)=\mathcal{D}^{\diamond \diamond_{0} ;+}(U)+\partial^{+}[g(U)]
$$

In order to isolate the third derivative in $\mathcal{Y}_{2}^{+}$, we write

$$
\begin{aligned}
& \mathcal{Y}_{2 a}^{+}(U)=\mathcal{I}_{+}^{\diamond_{0} ;+}(U) \partial^{+} \partial^{(2)} U \\
& \mathcal{Y}_{2 b}^{+}(U)=\left[\mathcal{D}^{\diamond_{0} ;+}(U)-\mathcal{I}_{+}^{\diamond_{0} ;+}(U) \partial^{+} \partial^{(2)} U\right]+\partial^{+}[g(U)] .
\end{aligned}
$$

Our third main result shows that $\mathcal{G}^{+}(U)$ can be decomposed into the components

$$
\begin{aligned}
& \mathcal{G}_{A^{\prime} a}^{+}(U)=\left[1-\mathcal{Y}_{1}(U) \mathcal{X}_{A}(U)\right] \mathcal{Y}_{2 a}^{+}(U), \\
& \mathcal{G}_{A^{\prime} b}^{+}(U)=\left[1-\mathcal{Y}_{1}(U) \mathcal{X}_{A}(U)\right] \mathcal{Y}_{2 b}^{+}(U), \\
& \mathcal{G}_{A^{\prime} c}^{+}(U)=-\mathcal{Y}_{1}^{+}(U) \mathcal{X}_{A}(U) T^{+}\left[\mathcal{Y}_{2}(U)\right],
\end{aligned}
$$

together with

$$
\begin{aligned}
& \mathcal{G}_{B^{\prime}}^{+}(U)=\mathcal{Y}_{1}^{+}(U) T^{+} \sum_{-; h} \mathcal{Y}_{2}(U) T^{-}\left[\mathcal{X}_{B}(U) \mathcal{D}^{\diamond+;+}(U)\right], \\
& \mathcal{G}_{C^{\prime}}^{+}(U)=\mathcal{Y}_{1}^{+}(U) T^{+} \sum_{-; h} \mathcal{Y}_{2}(U) T^{-}\left[\mathcal{X}_{C}(U) \mathcal{D}^{\diamond_{0} ;+}(U)\right], \\
& \mathcal{G}_{D^{\prime}}^{+}(U)=\mathcal{Y}_{1}^{+}(U) T^{+} \sum_{-; h} \mathcal{Y}_{2}(U) T^{-}\left[\mathcal{X}_{D}(U) \mathcal{D}^{\diamond_{0} ;+}(U)\right] .
\end{aligned}
$$

Observe that the structure of these latter components strongly resembles those of their counterparts $\mathcal{G}_{B}$ through $\mathcal{G}_{D}$, which will be useful for the analysis in the sequel.

Proposition 6.3 Suppose that $(\mathrm{Hg})$ is satisfied and fix $0<\kappa<\frac{1}{12}$ and $h>0$. Then for any $U \in \Omega_{h ; \kappa}$ we have the identity

$$
\mathcal{G}^{+}(U)=\mathcal{G}_{A^{\prime} a}^{+}(U)+\mathcal{G}_{A^{\prime} b}^{+}(U)+\mathcal{G}_{A^{\prime} c}^{+}(U)+\mathcal{G}_{B^{\prime}}^{+}(U)+\mathcal{G}_{C^{\prime}}^{+}(U)+\mathcal{G}_{D^{\prime}}^{+}(U) .
$$

Our final main result links our decompositions for $\mathcal{G}$ and $\mathcal{G}^{+}$to the framework developed in $§ 5$. In particular, we define the set of nonlinearities

$$
\mathcal{S}_{\mathrm{nl}}=\left\{\mathcal{D}^{\diamond_{0}}, p, p^{\diamond_{0}}, p^{\diamond+}, \mathcal{D}^{\diamond_{0}}, \mathcal{D}^{\diamond_{0} ;+}, \mathcal{D}^{\diamond_{-} ;+}, \mathcal{Z}^{+}, \mathcal{Z}^{-}, g\right\}
$$

which all appear in the decomposition (6.10) for $\mathcal{G}$. In addition, for each $f \in \mathcal{S}_{\text {nl }}$ we define a set of preferred exponents $Q_{f ; \text { pref }} \subset\{2, \infty\}$ via

$$
Q_{f ; \text { pref }}= \begin{cases}\{2\} & \text { for } f \in\left\{\mathcal{D}^{\diamond_{0}}, \mathcal{D}^{\diamond_{0} ;+}, \mathcal{D}^{\diamond_{-} ;+}, g\right\}, \\ \{\infty\} & \text { for } f \in\left\{p, p^{\diamond_{0}}, p^{\diamond_{+}}, \mathcal{Z}^{+}, \mathcal{Z}^{-}\right\}, \\ \{2, \infty\} & \text { for } f \in\left\{\mathcal{D}^{\diamond_{0}}\right\} .\end{cases}
$$

These can be interpreted as the preferred set of norms that we wish to apply to our nonlinearities, which in most (but not all!) cases lead to benign contributions to our final errors; see $\$ 7.2$.

We now turn to the decomposition (6.16) for $\mathcal{G}^{+}$. Introducing the notation $g^{+}(U)=$ $\partial^{+} g(U)$, we also define

$$
\overline{\mathcal{S}}_{\mathrm{nl}}=\mathcal{S}_{\mathrm{nl}} \cup\left\{\tilde{p}, \mathcal{I}_{0 s}^{\diamond \diamond_{0} ;+}, \mathcal{I}_{s s}^{\diamond \diamond_{0} ;+}, g^{+}, \partial^{(2)}\right\},
$$


together with the preferred exponent sets

$$
\bar{Q}_{f ; \text { pref }}= \begin{cases}\{2\} & \text { for } f \in\left\{\mathcal{D}^{\diamond_{0}}, \mathcal{D}^{\diamond_{-} ;+}, g, g^{+}\right\}, \\ \{\infty\} & \text { for } f \in\left\{\widetilde{p}, p, p^{\diamond_{0}}, p^{\diamond_{+}}, \mathcal{I}_{0 s}^{\diamond_{0} ;+}, \mathcal{I}_{0 s}^{\diamond_{0} ;+}, \mathcal{Z}^{+}, \mathcal{Z}^{-}\right\}, \\ \{2, \infty\} & \text { for } f \in\left\{\mathcal{D}^{\diamond_{0}}, \partial^{(2)}, \mathcal{D}^{\diamond_{0} ;+}\right\} .\end{cases}
$$

Comparing with (6.18), we remark that $\infty$ was added to $Q_{\mathcal{D}^{\diamond}{ }^{;} ;}$; pref . This is motivated by the fact that the $\mathcal{G}_{A^{\prime} c}^{+}(U)$ term contains a product of this nonlinearity with $\mathcal{D}^{\diamond \diamond_{0}}$, forcing us to evaluate one of the two in $\ell_{h}^{\infty}$. In any case, we note that for any $f \in \mathcal{S}_{\mathrm{nl}}$ we have

$$
Q_{f ; \text { pref }} \subset \bar{Q}_{f ; \text { pref }} .
$$

Notice that we are excluding the third derivative from the set $\bar{S}_{\mathrm{nl}}$. Recalling the identity

$$
\mathcal{Z}^{-}(U) \mathcal{Z}^{+}(U)=1
$$

and using (4.32), we obtain the simplification

$$
\begin{aligned}
\mathcal{G}_{A^{\prime} a}^{+}(U) & =\left[1-\mathcal{D}^{\diamond_{0}}(U) p(U)\right] \mathcal{I}_{+}^{\diamond_{0} ;+}(U) \partial^{+} \partial^{(2)} U \\
& =\tilde{p}(U) \mathcal{I}_{+}^{\diamond_{0} ;+}(U) \partial^{+} \partial^{(2)} U .
\end{aligned}
$$

The third derivative requires special attention, but appears here in a relatively straightforward fashion. For this reason, we exclude it from our general statements here and analyze it directly in the sequel.

The following result states that $\mathcal{G}(U)$ and $\mathcal{G}^{+}(U)-\mathcal{G}_{A^{\prime} a}^{+}(U)$ can be decomposed into products of the two types discussed in $§ 5.1$. In addition, every product can be estimated in $\ell_{h}^{2}$ by only using norms $\|f(U)\|_{\ell_{h}^{q}}$ for which $q \in Q_{f \text {; pref }}$ respectively $q \in \bar{Q}_{f \text {; pref }}$.

Corollary 6.4 Fix $0<\kappa<\frac{1}{12}$ and $h>0$. There exists an integer $N>1$ together with integers $k_{i} \geq 1$, sequences of integers

$$
\begin{aligned}
& \mathbf{q}_{\pi_{i}}=\left(q_{\pi_{i} ; 1}, \ldots, q_{\pi_{i} ; k_{i}}\right) \in\{2, \infty\}^{k_{i}}, \\
& \overline{\mathbf{q}}_{\bar{\pi}_{i}}=\left(\bar{q}_{\bar{\pi}_{i} ; 1}, \ldots, \bar{q}_{\bar{\pi}_{i} ; k_{i}}\right) \in\{2, \infty\}^{k_{i}},
\end{aligned}
$$

sequences of functions

$$
\begin{aligned}
\mathbf{f}_{i} & =\left(f_{i ; 1}, \ldots, f_{i ; k_{i}}\right) \in \mathcal{S}_{\mathrm{nl}}^{k_{i}} \\
\overline{\mathbf{f}}_{i} & =\left(\bar{f}_{i ; 1}, \ldots, \bar{f}_{i ; k_{i}}\right) \in \overline{\mathcal{S}}_{\mathrm{nl}}^{k_{i}}
\end{aligned}
$$

and maps

$$
\pi_{i}: \ell_{h}^{\mathbf{q}_{\pi_{i}}} \rightarrow \ell_{h}^{2}, \quad \bar{\pi}_{i}: \ell_{h}^{\overline{\mathbf{q}}_{\bar{\pi}_{i}}} \rightarrow \ell_{h}^{2},
$$

each defined for $1 \leq i \leq N$, so that the following properties hold true.

(i) For each $U \in \Omega_{h ; \kappa}$ we have the decomposition

$$
\begin{aligned}
\mathcal{G}(U) & =\sum_{i=1}^{N} \pi_{i}\left[f_{i ; 1}(U), \ldots, f_{i ; k_{i}}(U)\right] \\
\mathcal{G}^{+}(U)-\mathcal{G}_{A^{\prime} a}^{+}(U) & =\sum_{i=1}^{N} \bar{\pi}_{i}\left[\bar{f}_{i ; 1}(U), \ldots, \bar{f}_{i ; k_{i}}(U)\right] .
\end{aligned}
$$


(ii) For each $1 \leq i \leq N$ the pairs $\left(\mathbf{q}_{\pi_{i}}, \pi_{i}\right)$ and $\left(\overline{\mathbf{q}}_{\bar{\pi}_{i}}, \bar{\pi}_{i}\right)$ both satisfy the conditions of either Lemma 5.1 or Lemma 5.2.

(iii) For each $1 \leq i \leq N$ and $1 \leq j \leq k_{i}$ we have the inclusions

$$
q_{\pi_{i} ; j} \in Q_{f_{i ; j} ; \text { pref }}, \quad \bar{q}_{\bar{\pi}_{i} ; j} \in \bar{Q}_{\bar{f}_{i ; j} ; \text { pref }},
$$

Proof of Corollary 6.4 The desired decomposition can be read off directly from the structure of the terms defined in (6.9), (6.14) and (6.15).

\subsection{Summation by Parts}

Our goal here is to establish Propositions 6.1-6.3 by using a summation by parts procedure to transfer the discrete derivative in (4.8) from the dangerous second-order term $\mathcal{D}^{\diamond \diamond_{0}}$ to the better-behaved product $\mathcal{X}_{A}(U)=p(U) \mathcal{Z}^{+}(U)$; see (4.2) and (4.4). Lemma 6.5 shows how the auxiliary functions $\mathcal{X}_{B}, \mathcal{X}_{C}$ and $\mathcal{X}_{D}$ introduced in (6.8) arise naturally through this process.

Proof of Proposition 6.1 The inclusions follow directly from Lemma's D.5 and D.7 . In addition, we may use (3.8) to compute

$$
\begin{aligned}
& \partial^{+} p(U)=\partial^{+}\left[\frac{\mathcal{D}^{\diamond}(U)}{1+\mathcal{D}^{\diamond}(U) \mathcal{D}^{\diamond_{0}}(U)}\right] \\
& =\frac{S^{+}\left[1+\mathcal{D}^{\diamond}(U) \mathcal{D}^{\diamond_{0}}(U)\right] \partial^{+}\left[\mathcal{D}^{\diamond}(U)\right]}{P^{+}\left[1+\mathcal{D}^{\diamond}(U) \mathcal{D}^{\diamond_{0}}(U)\right]}-\frac{S^{+}\left[\mathcal{D}^{\diamond}(U)\right] \partial^{+}\left[\mathcal{D}^{\diamond}(U) \mathcal{D}^{\diamond_{0}}(U)\right]}{P^{+}\left[1+\mathcal{D}^{\diamond+}(U) \mathcal{D}^{\diamond_{0}}(U)\right]} \\
& =\left[P^{+} \tilde{p}(U)\right]^{-1} S^{+}\left[1+\mathcal{D}^{\diamond}(U) \mathcal{D}^{\diamond_{0}}(U)\right] \mathcal{D}^{\diamond+;+}(U) \\
& -\left[P^{+} \widetilde{p}(U)\right]^{-1} S^{+}\left[\mathcal{D}^{\diamond}(U)\right] \partial^{+}\left[\mathcal{D}^{\diamond+}(U) \mathcal{D}^{\diamond}(U)\right] .
\end{aligned}
$$

Applying (3.8) once more we obtain the desired decomposition

$$
\begin{aligned}
\partial^{+} p(U)= & {\left[P^{+} \tilde{p}(U)\right]^{-1} S^{+}\left[1+\mathcal{D}^{\diamond}(U) \mathcal{D}^{\diamond_{0}}(U)\right] \mathcal{D}^{\diamond+;}(U) } \\
& -\left[P^{+} \tilde{p}(U)\right]^{-1} S^{+}\left[\mathcal{D}^{\diamond}(U)\right] S^{+}\left[\mathcal{D}^{\diamond_{0}}(U)\right] \mathcal{D}^{\diamond_{+} ;+}(U) \\
& -\left[P^{+} \tilde{p}(U)\right]^{-1} S^{+}\left[\mathcal{D}^{\diamond}(U)\right] S^{+}\left[\mathcal{D}^{\diamond}(U)\right] \mathcal{D}^{\diamond_{0} ;+}(U) .
\end{aligned}
$$

Lemma 6.5 Fix $0<\kappa<\frac{1}{12}$ and $h>0$. Then for any $U \in \Omega_{h ; \kappa}$ we have the identity

$$
\partial^{+}\left[\mathcal{X}_{A}(U)\right]=\mathcal{X}_{B}(U) \mathcal{D}^{\diamond+;+}(U)+\mathcal{X}_{C}(U) \mathcal{D}^{\diamond_{0} ;+}(U)+\mathcal{X}_{D}(U) \mathcal{D}^{\diamond_{0} ;+}(U) .
$$

Proof Applying (3.8) and (4.26), we compute

$$
\begin{aligned}
\partial^{+}\left[\mathcal{X}_{A}(U)\right]= & \partial^{+}\left[p(U) \mathcal{Z}^{+}(U)\right] \\
= & \partial^{+}[p(U)] S^{+}\left[\mathcal{Z}^{+}(U)\right]+S^{+}[p(U)] \partial^{+}\left[\mathcal{Z}^{+}(U)\right] \\
= & {\left[p_{A}^{\diamond_{+}}(U)+p_{B}^{\diamond_{+}}(U)\right] \mathcal{D}^{\diamond+;}(U) S^{+}\left[\mathcal{Z}^{+}(U)\right] } \\
& +p^{\diamond_{0}}(U) \mathcal{D}^{\diamond_{0} ;+}(U) S^{+}\left[\mathcal{Z}^{+}(U)\right] \\
& +S^{+}[p(U)] p(U) \mathcal{Z}^{+}(U) \mathcal{D}^{\diamond_{0} ;+}(U),
\end{aligned}
$$

which yields the desired result. 
Proof of Proposition 6.2 Applying the discrete summation-by-parts formula (3.13) to the expression (4.8) for $\mathcal{Y}$, we obtain

$$
\begin{aligned}
\mathcal{Y}(U)= & -\mathcal{Z}^{-}(U) T^{-}\left[p(U) \mathcal{Z}^{+}(U)\right]\left[\mathcal{D}^{\diamond_{0}}(U)+g(U)\right] \\
& +\mathcal{Z}^{-}(U) \sum_{-; h}\left[\mathcal{D}^{\diamond_{0}}(U)+g(U)\right] \partial^{-}\left[p(U) \mathcal{Z}^{+}(U)\right] .
\end{aligned}
$$

Exploiting the definitions (6.7)-(6.8), this allows us to write

$$
\mathcal{G}(U)=-\mathcal{Y}_{1}(U) \mathcal{Y}_{2}(U) T^{-}\left[\mathcal{X}_{A}(U)\right]+\mathcal{Y}_{1}(U) \sum_{-; h} \mathcal{Y}_{2}(U) \partial^{-} \mathcal{X}_{A}(U)+\mathcal{Y}_{2}(U)
$$

Applying (6.31), we find

$$
\begin{aligned}
\mathcal{G}(U)= & {\left[1-\mathcal{Y}_{1}(U) T^{-}\left[\mathcal{X}_{A}(U)\right]\right] \mathcal{Y}_{2}(U) } \\
& +\mathcal{Y}_{1}(U) \sum_{-; h} \mathcal{Y}_{2}(U) T^{-}\left[\mathcal{X}_{B}(U) \mathcal{D}^{\diamond+;}(U)\right] \\
& +\mathcal{Y}_{1}(U) \sum_{-; h} \mathcal{Y}_{2}(U) T^{-}\left[\mathcal{X}_{C}(U) \mathcal{D}^{\diamond_{0} ;+}(U)+\mathcal{X}_{D}(U) \mathcal{D}^{\diamond_{0} ;+}(U)\right],
\end{aligned}
$$

as desired.

Proof of Proposition 6.3 We use the preliminary expression (6.34) together with (3.5) to compute

$$
\begin{aligned}
\partial^{+}[\mathcal{G}(U)]= & -\mathcal{Y}_{1}^{+}(U) T^{+}\left[\mathcal{Y}_{2}(U)\right] \mathcal{X}_{A}(U)-\mathcal{Y}_{1}(U) \mathcal{Y}_{2}^{+}(U) \mathcal{X}_{A}(U)-\mathcal{Y}_{1}(U) \mathcal{Y}_{2}(U) \partial^{-}\left[\mathcal{X}_{A}(U)\right] \\
& +\mathcal{Y}_{1}^{+}(U) T^{+} \sum_{-; h} \mathcal{Y}_{2}(U) \partial^{-} \mathcal{X}_{A}+\mathcal{Y}_{1}(U) \mathcal{Y}_{2}(U) \partial^{-} \mathcal{X}_{A} \\
& +\mathcal{Y}_{2}^{+}(U) \\
= & -\mathcal{Y}_{1}^{+}(U) \mathcal{X}_{A}(U) T^{+} \mathcal{Y}_{2}(U)+\left(1-\mathcal{Y}_{1}(U) \mathcal{X}_{A}(U)\right) \mathcal{Y}_{2}^{+}(U) \\
& +\mathcal{Y}_{1}^{+}(U) T^{+} \sum_{-; h} \mathcal{Y}_{2}(U) \partial^{-} \mathcal{X}_{A}
\end{aligned}
$$

Applying (6.31) now yields the desired decomposition.

\section{Component Estimates}

Our goal in this section is to introduce the terminology that allows the conditions $(h f),(h f)_{\text {lin }}$ and $(h f)_{\mathrm{nl}}$ in $\$ 5.2$ to be verified for the nonlinearities $f \in \mathcal{S}_{\mathrm{nl}} \cup \bar{S}_{\mathrm{nl}}$ introduced in (6.17) and (6.19). In particular, we construct suitable approximants $f_{\text {apx }}$ and $f_{\text {lin }}$ that are accurate to leading order in $h$, but also tractable to use in our subsequent computations.

In order to apply Proposition 5.4 in a streamlined fashion, we state our estimates that are relevant for (5.49) in terms of the quantities

$$
\begin{aligned}
S_{\text {full }}(V) & =\|V\|_{\ell_{h}^{2 ; 2}}+\left\|\partial^{+} V\right\|_{\ell_{h}^{\infty}}, \\
\bar{S}_{\text {full }}(V) & =S_{\text {full }}(V)+\left\|\partial^{+} \partial^{+} V\right\|_{\ell_{h}^{\infty}}, \\
S_{2 ; \text { fix }}(V) & =\|V\|_{\ell_{h}^{2 ; 2}}, \\
\bar{S}_{2 ; \text { fix }}(V) & =S_{2 ; \text { fix }}(V)
\end{aligned}
$$


related to the seminorms in condition $(h f)$. Roughly speaking, $S_{\text {full }}$ correponds with the whole set of seminorms that we need to use, while $S_{2 \text {;ix }}$ reflects the contribution of nonlinearities where we only want to apply (5.26) and (5.27) with the exponent $q=2$. In addition, we introduce the expressions

$$
\begin{array}{lll}
T_{\text {safe }}(V)=\|V\|_{\ell_{h}^{2 ; 2}}, & & \bar{T}_{\text {safe }}(V)=T_{\text {safe }}(V), \\
T_{\infty ; \text { opt }}(V)=\left\|\partial^{+} V\right\|_{\ell_{h}^{\infty}}, & & \bar{T}_{\infty ; \text { opt }}(V)=T_{\infty ; \text { opt }}(V)+\left\|\partial^{+} \partial^{+} V\right\|_{\ell_{h}^{\infty}}
\end{array}
$$

associated to the linear terms in condition $(h f)_{\text {lin }}$. Here $T_{\infty ; \text { opt }}$ represents the contributions of $\left\|f_{\operatorname{lin} ; U}^{A}[V]\right\|_{\ell_{h}^{\infty}}$ and $\left\|f_{\operatorname{lin} ; U}^{B}[V]\right\|_{\ell_{h}^{\infty}}$ where the use of the supremum norm is optional, in the sense that they could also be measured in $\ell_{h}^{2}$. The remaining contributions are all reflected in $T_{\text {safe. }}$. Finally, we use the functions

$$
\begin{aligned}
& \mathcal{E}_{\mathrm{nl}}(V)=\left(\|V\|_{\ell_{h}^{2 ; 2}}+\|V\|_{\ell_{h}^{\infty ; 1}}+h\right)\|V\|_{\ell_{h}^{\ell^{2 ; 2}}}, \\
& \overline{\mathcal{E}}_{\mathrm{nl}}(V)=\mathcal{E}_{\mathrm{nl}}(V)
\end{aligned}
$$

to control the nonlinear terms (5.48), while enforcing the approximate bound

$$
\left\|f(U)-f_{\text {apx }}(U)\right\|_{\ell_{h}^{q}} \leq K h \quad \text { for all } \quad(q, U, h) \in Q_{f} \times \Omega_{h ; \kappa} \times \mathbb{R}_{>0} .(7.4
$$

We divide our nonlinearities into five distinct groups that are fully described by Propositions 7.1-7.5 in $§ 7.1$. In $\$ 7.2$ we subsequently discuss a number of bookkeeping issues that in $\S 8$ will allow us to control the cross-terms $(5.49)$ for $\mathcal{G}(U)$ by

$$
\mathcal{J}_{\text {cross } ;}(V)=T_{\text {safe }}(V) S_{\text {full }}(V)+T_{\infty ; \text { opt }}(V) S_{2 ; \text { fix }}(V) .
$$

Naturally, the related estimate for $\mathcal{G}^{+}(U)-\mathcal{G}_{A^{\prime} a}^{+}(U)$ will also hold. The reader should keep in mind that products of $\ell_{h}^{\infty}$ bounds will turn out to be very dangerous in [32], which is why we go to such great lengths here to avoid them. More specifically, we want to exclude contributions of the form $T_{\infty ; \text { opt }}(V) S_{\text {full }}(V)$ to the cross-terms.

\subsection{Estimates}

In order to simplify the notation and break the directional biases associated to the $\partial^{+}$and $\partial^{-}$ terms that appear in the definitions (3.16) for the gridspace functions $r_{U}^{ \pm}$, we introduce the sequence

$$
\gamma_{U}=\sqrt{1-\left(\partial^{0} U\right)^{2}}
$$

Most of the approximants $f_{\text {apx }}(U)$ that we introduce below arise from the simple replacements $\partial^{ \pm} U \mapsto \partial^{0} U$ and $r_{U}^{ \pm} \mapsto \gamma_{U}$, but we have to carefully track shifted second derivatives. In addition, $f_{\text {lin; } U}$ can almost always be interpreted as a direct linearization of $f_{\text {apx }}(U)$, but the terms $\mathcal{Z}^{ \pm}$require special care. We note that the point of the results below is not only to provide the actual estimates, but also to catalog in which spaces the estimates are available; see $\$ 7.2$.

The first set of nonlinearities is given by the singleton

$$
\mathcal{S}_{\mathrm{nl} ; I}=\left\{\mathcal{D}^{\diamond_{0}}\right\},
$$

see (3.17). We define

$$
\mathcal{D}_{\text {apx }}^{\diamond_{0}}(U)=\gamma_{U}^{-1} \partial^{0} U, \quad \mathcal{D}_{\operatorname{lin} ; U}^{\diamond_{0}}[V]=\gamma_{U}^{-3} \partial^{0} V .
$$


For any $f \in \mathcal{S}_{\mathrm{nl} ; I}$, we write

$$
Q_{f}=\{2, \infty\}, \quad Q_{f ; \text { lin }}^{A}=Q_{f ; \text { lin }}^{B}=\{2, \infty\}, \quad Q_{f ; \mathrm{nl}}^{A}=Q_{f ; \mathrm{nl}}^{B}=\{2\}
$$

and recall that $Q_{f ; \text { pref }}=\bar{Q}_{f ; \text { pref }}=\{2, \infty\}$.

Proposition 7.1 (see §F.2) Assume that $(\mathrm{Hg})$ is satisfied, fix $0<\kappa<\frac{1}{12}$ and pick any nonlinearity $f \in \mathcal{S}_{\mathrm{nl} ; I}$. Then there exists a constant $K>0$ so that (7.4) holds and the following properties are true.

(i) Upon introducing the seminorms

$$
\begin{gathered}
{[V]_{f ; 2, h}=\left\|\partial^{+} V\right\|_{\ell_{h}^{2}} \leq S_{\text {full }}(V),} \\
{[V]_{f ; \infty, h}=\left\|\partial^{+} V\right\|_{\ell_{h}^{\infty}} \leq S_{\text {full }}(V),}
\end{gathered}
$$

the conditions in $(h f)$ are all satisfied.

(ii) Upon writing $f_{\operatorname{lin} ; U}^{B}=0$, the conditions in $(h f)_{\text {lin }}$ are satisfied. In addition, the bounds

$$
\begin{aligned}
& \left\|f_{\text {lin;U }}^{A}[V]\right\|_{\ell_{h}^{2}} \leq K\left\|\partial^{+} V\right\|_{\ell_{h}^{2}} \leq K T_{\text {safe }}(V), \\
& \left\|f_{\text {lin;U }}^{A}[V]\right\|_{\ell_{h}^{\infty}} \leq K\left\|\partial^{+} V\right\|_{\ell_{h}^{\infty}} \leq K T_{\infty ; \text { opt }}(V)
\end{aligned}
$$

hold for all $U \in \Omega_{h ; \kappa}, h>0$ and $V \in \ell_{h}^{2}$.

(iii) Upon writing $f_{\mathrm{nl} ; U}^{B}=0$, the conditions in $(h f)_{\mathrm{nl}}$ are satisfied. In addition, we have the bound

$$
\begin{aligned}
\left\|f_{\mathrm{nl} ; U}^{A}(V)\right\|_{\ell_{h}^{2}} & \leq K\left\|\partial^{+} V\right\|_{\infty}\left\|\partial^{+} V\right\|_{\ell_{h}^{2}}+K h\left[\left\|\partial^{+} V\right\|_{\ell_{h}^{2}}+\left\|\partial^{+} \partial^{+} V\right\|_{\ell_{h}^{2}}\right] \\
& \leq K \mathcal{E}_{\mathrm{nl}}(V)
\end{aligned}
$$

for all $U \in \Omega_{h ; \kappa}, h>0$ and $V \in \ell_{h}^{2}$.

The second set of nonlinearities is given by

$$
\mathcal{S}_{\mathrm{nl} ; I I}=\left\{\tilde{p}, p, p^{\diamond_{0}}, p^{\diamond_{+}}, \mathcal{I}_{0 s}^{\diamond_{0} ;+}, \mathcal{I}_{s s}^{\diamond_{0} ;+}, \mathcal{I}_{+}^{\diamond_{0} ;+}\right\},
$$

see (3.28), (4.2) and (6.3). We remark that $\mathcal{I}_{+}^{\diamond \diamond_{0} ;+} \notin \overline{\mathcal{S}}_{\text {nl }}$, but we do need the bounds stated below in order to estimate $\mathcal{G}_{A^{\prime} a}^{+}$. We write

$$
\begin{aligned}
& \tilde{p}_{\text {apx }}(U)=\gamma_{U}^{2}, \quad \tilde{p}_{\text {lin } ; U}[V]=-2 \partial^{0} U \partial^{0} V, \\
& p_{\text {apx }}(U)=\gamma_{U} \partial^{0} U, \quad p_{\text {lin; } U}[V]=\gamma_{U}^{-1}\left(2 \gamma_{U}^{2}-1\right) \partial^{0} V, \\
& p_{\text {apx }}^{\diamond 0}(U)=\gamma_{U}^{2}\left(\gamma_{U}^{2}-1\right), \quad p_{\text {lin; } U}^{\diamond_{\diamond}[V]}=\left(2-4 \gamma_{U}^{2}\right) \partial^{0} U \partial^{0} V \text {, } \\
& p_{\text {apx }}^{\diamond+}(U)=\gamma_{U}^{4}, \quad p_{\text {lin; } U}^{\diamond+}[V]=-4 \gamma_{U}^{2} \partial^{0} U \partial^{0} V \text {, } \\
& \mathcal{I}_{0 s ; \text { apx }}^{\diamond \diamond_{0} ;+}(U)=2 \gamma_{U}^{-6} \partial^{0} U, \quad \mathcal{I}_{0 s ; \text { lin; } U}^{\diamond \diamond_{0} ;+}[V]=2\left[6 \gamma_{U}^{-8}-5 \gamma_{U}^{-6}\right] \partial^{0} V \text {, } \\
& \mathcal{I}_{s s ; \text { apx }}^{\diamond \diamond_{0} ;+}(U)=2 \gamma_{U}^{-6} \partial^{0} U, \quad \mathcal{I}_{s s ; \text { lin; } U}^{\diamond \diamond_{0} ;+}[V]=2\left[6 \gamma_{U}^{-8}-5 \gamma_{U}^{-6}\right] \partial^{0} V \text {, } \\
& \mathcal{I}_{+; \text {apx }}^{\diamond \diamond_{0} ;+}(U)=\gamma_{U}^{-4}, \quad \mathcal{I}_{+; \text {lin; } U}^{\diamond \diamond_{0} ;+}[V]=4 \gamma_{U}^{-6} \partial^{0} U \partial^{0} V .
\end{aligned}
$$

In addition, we write

$$
Q_{f}=\{\infty\}, \quad Q_{f ; \text { lin }}^{A}=Q_{f ; \text { lin }}^{B}=\{2, \infty\}, \quad Q_{f ; \mathrm{nl}}^{A}=Q_{f ; \mathrm{nl}}^{B}=\{2\}
$$

for each $f \in \mathcal{S}_{\mathrm{nl} ; I I}$. We recall that $Q_{f \text {;pref }}=\{\infty\}$ for $f \in \mathcal{S}_{\mathrm{nl} ; I I} \cap \mathcal{S}_{\mathrm{nl}}$ and $\bar{Q}_{f ; \text { pref }}=\{\infty\}$ for $f \in \mathcal{S}_{\mathrm{nl} ; I I} \cap \overline{\mathcal{S}}_{\mathrm{nl}}$. 
For later use, we recall the definitions (3.29) and remark that we can formally write

$$
\begin{aligned}
& \mathcal{D}_{a ; \text { apx }}^{\diamond_{0} ;+}(U)=\gamma_{U}^{-4} \partial^{+} \partial^{(2)} U, \\
& \mathcal{D}_{b ; \text { apx }}^{\diamond \diamond_{0} ;+}(U)=2 \gamma_{U}^{-6} \partial^{0} U S^{+}\left[\partial^{(2)} U\right] T^{+}\left[\partial^{(2)} U\right],
\end{aligned}
$$

together with

$$
\begin{aligned}
\mathcal{D}_{a ; l i n ; U}^{\diamond \diamond_{0} ;+}[V]= & 4 \gamma_{U}^{-6} \partial^{0} U\left[\partial^{+} \partial^{(2)} U\right] \partial^{0} V+\gamma_{U}^{-4} \partial^{+} \partial^{(2)} V \\
\mathcal{D}_{b ; \operatorname{lin} ; U}^{\diamond \otimes_{0}}[V]= & 4\left[6 \gamma_{U}^{-8}-5 \gamma_{U}^{-6}\right] S^{+}\left[\partial^{(2)} U\right] T^{+}\left[\partial^{(2)} U\right] \partial^{0} V \\
& +4 \gamma_{U}^{-6} \partial^{0} U\left[T^{+}\left[\partial^{(2)} U\right] S^{+}\left[\partial^{(2)} V\right]+S^{+}\left[\partial^{(2)} U\right] T^{+}\left[\partial^{(2)} V\right]\right] .
\end{aligned}
$$

Proposition 7.2 (see §F.5) Assume that $(\mathrm{Hg})$ is satisfied, fix $0<\kappa<\frac{1}{12}$ and pick any nonlinearity $f \in \mathcal{S}_{\mathrm{nl} ; I I}$. Then there exists a constant $K>0$ so that (7.4) holds and the following properties are true.

(i) Upon introducing the seminorm

$$
[V]_{f ; \infty, h}=\left\|\partial^{+} V\right\|_{\ell_{h}^{\infty}} \leq S_{\text {full }}(V),
$$

the conditions in $(h f)$ are all satisfied.

(ii) Upon writing $f_{\operatorname{lin} ; U}^{B}=0$, the conditions in $(h f)_{\operatorname{lin}}$ are satisfied. In addition, the bounds

$$
\begin{aligned}
& \left\|f_{\operatorname{lin} ; U}^{A}[V]\right\|_{\ell_{h}^{2}} \leq K\left\|\partial^{+} V\right\|_{\ell_{h}^{2}} \leq K T_{\text {safe }}(V), \\
& \left\|f_{\operatorname{lin} ; U}^{A}[V]\right\|_{\ell_{h}^{\infty}} \leq K\left\|\partial^{+} V\right\|_{\ell_{h}^{\infty}} \leq K T_{\infty ; \mathrm{opt}}(V)
\end{aligned}
$$

hold for all $U \in \Omega_{h ; \kappa}, h>0$ and $V \in \ell_{h}^{2}$.

(iii) Upon writing $f_{\mathrm{nl} ; U}^{B}=0$, the conditions in $(h f)_{\mathrm{nl}}$ are satisfied. In addition, we have the bound

$$
\begin{aligned}
\left\|f_{\mathrm{nl} ; U}^{A}(V)\right\|_{\ell_{h}^{2}} & \leq K\left\|\partial^{+} V\right\|_{\ell_{h}^{\infty}}\left\|\partial^{+} V\right\|_{\ell_{h}^{2}}+K h\left[\left\|\partial^{+} V\right\|_{\ell_{h}^{2}}+\left\|\partial^{+} \partial^{+} V\right\|_{\ell_{h}^{2}}\right] \\
& \leq K \mathcal{E}_{\mathrm{nl}}(V)
\end{aligned}
$$

for all $U \in \Omega_{h ; \kappa}, h>0$ and $V \in \ell_{h}^{2}$.

The third set of nonlinearities is given by

$$
\mathcal{S}_{\mathrm{nl} ; I I I}=\left\{\mathcal{D}^{\diamond_{0}}, \mathcal{D}^{\diamond_{0} ;+}, \mathcal{D}^{\diamond-;+}, \partial^{(2)}\right\}
$$

see (3.21) and (3.26). We write

$$
\begin{aligned}
& \mathcal{D}_{\text {apx }}^{\diamond \diamond 0}(U)=\gamma_{U}^{-4} \partial^{(2)} U, \quad \mathcal{D}_{\operatorname{lin} ; U}^{\diamond 00}[V] \quad=4 \gamma_{U}^{-6} \partial^{0} U\left[\partial^{(2)} U\right] \partial^{0} V+\gamma_{U}^{-4} \partial^{(2)} V, \\
& \mathcal{D}_{\text {apx }}^{\diamond ;+}(U)=\gamma_{U}^{-3} S^{+}\left[\partial^{(2)} U\right], \quad \mathcal{D}_{\operatorname{lin} ; U}^{\diamond_{0} ;+}[V]=3 \gamma_{U}^{-5} \partial^{0} U S^{+}\left[\partial^{(2)} U\right] \partial^{0} V+\gamma_{U}^{-3} S^{+}\left[\partial^{(2)} V\right], \\
& \mathcal{D}_{\text {apx }}^{\diamond ;+}(U)=\gamma_{U}^{-3} \partial^{(2)} U, \quad \mathcal{D}_{\text {lin; }: U}^{\diamond ;+}[V]=3 \gamma_{U}^{-5} \partial^{0} U\left[\partial^{(2)} U\right] \partial^{0} V+\gamma_{U}^{-3} \partial^{(2)} V, \\
& {\left[\partial^{(2)}\right] \text { apx }(U)=\partial^{(2)} U, \quad\left[\partial^{(2)}\right] \operatorname{lin} ; U[V]=\partial^{(2)} V .}
\end{aligned}
$$

In addition, for each $f \in \mathcal{S}_{\mathrm{nl} ; I I I}$ we write

$$
Q_{f}=\{2, \infty\}, \quad Q_{f ; \text { lin }}^{A}=Q_{f ; \text { lin }}^{B}=\{2, \infty\}, \quad Q_{f ; \mathrm{nl}}^{A}=Q_{f ; \mathrm{nl}}^{B}=\{2\} .
$$

We recall that $Q_{f \text {; pref }}=\bar{Q}_{f \text {; pref }}=\{2\}$ for $f \in\left\{\mathcal{D}^{\diamond_{0}}, \mathcal{D}^{\diamond_{-} ;+}\right\}$. For $f=\mathcal{D}^{\diamond_{0} ;+}$ we have $Q_{f ; \text { pref }}=\{2\}$ and for $f \in\left\{\mathcal{D}^{\diamond_{0} ;+}, \partial^{(2)}\right\}$ we have $\bar{Q}_{f ; \text { pref }}=\{2, \infty\}$. 
Proposition 7.3 (see §F.3) Assume that $(\mathrm{Hg})$ is satisfied, fix $0<\kappa<\frac{1}{12}$ and pick any nonlinearity $f \in \mathcal{S}_{\mathrm{nl} ; I I I}$. Then there exists a constant $K>0$ so that (7.4) holds and the following properties are true.

(i) Upon introducing the seminorms

$$
\begin{aligned}
{[V]_{f ; 2, h} } & =\left\|\partial^{+} V\right\|_{\ell_{h}^{2}}+\left\|\partial^{+} \partial^{+} V\right\|_{\ell_{h}^{2}} \\
& \leq \min \left\{S_{\text {full }}(V), S_{2 ; \text { fix }}(V)\right\}, \\
{[V]_{f ; \infty, h} } & =\left\|\partial^{+} V\right\|_{\ell_{h}^{\infty}}+\left\|\partial^{+} \partial^{+} V\right\|_{\ell_{h}^{\infty}} \\
& \leq \bar{S}_{\text {full }}(V),
\end{aligned}
$$

the conditions in $(h f)$ are all satisfied.

(ii) Upon writing $f_{\operatorname{lin} ; U}^{B}=0$, the conditions in $(h f)_{\text {lin }}$ are satisfied. In addition, the bounds

$$
\begin{aligned}
& \left\|f_{\operatorname{lin} ; U}^{A}[V]\right\|_{\ell_{h}^{2}} \leq K\left[\left\|\partial^{+} V\right\|_{\ell_{h}^{2}}+\left\|\partial^{+} \partial^{+} V\right\|_{\ell_{h}^{2}}\right] \leq K T_{\text {safe }}(V), \\
& \left\|f_{\operatorname{lin} ; U}^{A}[V]\right\|_{\ell_{h}^{\infty}} \leq K\left[\left\|\partial^{+} V\right\|_{\ell_{h}^{\infty}}+\left\|\partial^{+} \partial^{+} V\right\|_{\ell_{h}^{\infty}}\right] \leq K \bar{T}_{\infty ; \text { opt }}(V)
\end{aligned}
$$

hold for all $U \in \Omega_{h ; \kappa}, h>0$ and $V \in \ell_{h}^{2}$.

(iii) Upon writing $f_{\mathrm{nl} ; U}^{B}=0$, the conditions in $(h f)_{\mathrm{nl}}$ are satisfied. In addition, we have the bound

$$
\begin{aligned}
\left\|f_{\mathrm{nl} ; U}^{A}(V)\right\|_{\ell_{h}^{2}} \leq & K\left\|\partial^{+} V\right\|_{\infty}\left[\left\|\partial^{+} V\right\|_{\ell_{h}^{2}}+\left\|\partial^{+} \partial^{+} V\right\|_{\ell_{h}^{2}}\right] \\
& \quad+K h\left[\left\|\partial^{+} V\right\|_{\ell_{h}^{2}}+\left\|\partial^{+} \partial^{+} V\right\|_{\ell_{h}^{2}}\right] \\
\leq & K \mathcal{E}_{\mathrm{nl}}(V)
\end{aligned}
$$

for all $U \in \Omega_{h ; \kappa}, h>0$ and $V \in \ell_{h}^{2}$.

The fourth set of nonlinearities is given by

$$
\mathcal{S}_{\mathrm{nl} ; I V}=\left\{\mathcal{Z}^{+}, \mathcal{Z}^{-}\right\} .
$$

Here the approximations are considerably more delicate on account of the sum in the definitions (4.4); see §G for the details. Upon introducing the function

$$
\mathcal{E}_{\mathrm{sm}}(U)=h \partial^{-}\left[\gamma_{U}^{-4}\left(2-\gamma_{U}^{2}\right) S^{+}\left[\partial^{(2)} U\right]\right],
$$

which measures the smoothness of $U$ in some sense, we write

$$
\begin{array}{ll}
\mathcal{Z}_{\text {apx }}^{+}(U)=\gamma_{U}^{-1}, & \mathcal{Z}_{\text {lin } ; U}^{+}[V]=\gamma_{U}^{-3} \partial^{0} U \partial^{0} V+\gamma_{U}^{-1} \sum_{-; h} \mathcal{E}_{\mathrm{sm}}(U) \partial^{0} V, \\
\mathcal{Z}_{\text {apx }}^{-}(U)=\gamma_{U}, & \mathcal{Z}_{\text {lin } ; U}^{-}[V]=-\gamma_{U}^{-1} \partial^{0} U \partial^{0} V-\gamma_{U} \sum_{-; h} \mathcal{E}_{\mathrm{sm}}(U) \partial^{0} V .
\end{array}
$$

In addition, for every $f \in \mathcal{S}_{\mathrm{nl} ; I V}$ we write

$$
Q_{f}=\{\infty\}, \quad Q_{f ; \operatorname{lin}}^{A}=\{\infty\}, \quad Q_{f ; \text { lin }}^{B}=\{2, \infty\}, \quad Q_{f ; \mathrm{nl}}^{A}=\{\infty\}, \quad Q_{f ; \mathrm{nl}}^{B}=\{2\}
$$

and recall that $Q_{f ; \text { pref }}=\bar{Q}_{f ; \text { pref }}=\{\infty\}$.

Proposition 7.4 (see $\S \mathrm{G})$ Assume that $(\mathrm{Hg})$ is satisfied, fix $0<\kappa<\frac{1}{12}$ and pick any nonlinearity $f \in \mathcal{S}_{\mathrm{nl} ; I V}$. Then there exists a constant $K>0$ so that (7.4) holds and the following properties are true. 
(i) Upon introducing the seminorm

$$
[V]_{f ; \infty, h}=\left\|\partial^{+} V\right\|_{\ell_{h}^{2}}+\left\|\partial^{+} V\right\|_{\ell_{h}^{\infty}}+\left\|\partial^{+} \partial^{+} V\right\|_{\ell_{h}^{2}} \leq S_{\text {full }}(V),
$$

the conditions in $(h f)$ are all satisfied.

(ii) The conditions in $(h f)_{\text {lin }}$ are satisfied. In addition, the bounds

$$
\begin{aligned}
& \left\|f_{\operatorname{lin} ; U}^{A}[V]\right\|_{\ell_{h}^{\infty}} \leq K\left\|\partial^{+} V\right\|_{\ell_{h}^{2}} \leq K T_{\text {safe }}(V), \\
& \left\|f_{\operatorname{lin} ; U}^{B}[V]\right\|_{\ell_{h}^{2}} \leq K\left\|\partial^{+} V\right\|_{\ell_{h}^{2}} \leq K T_{\text {safe }}(V), \\
& \left\|f_{\operatorname{lin} ; U}^{B}[V]\right\|_{\ell_{h}^{\infty}} \leq K\left\|\partial^{+} V\right\|_{\ell_{h}^{\infty}} \leq K T_{\infty ; \text { opt }}(V)
\end{aligned}
$$

hold for all $U \in \Omega_{h ; \kappa}, h>0$ and $V \in \ell_{h}^{2}$.

(iii) The conditions in $(h f)_{\mathrm{nl}}$ are satisfied. In addition, we have the bounds

$$
\begin{aligned}
\left\|f_{\mathrm{nl} ; U}^{A}(V)\right\|_{\ell_{h}^{\infty}} \leq & K\left[\left\|\partial^{+} V\right\|_{\ell_{h}^{2}}^{2}+\left\|\partial^{+} \partial^{+} V\right\|_{\ell_{h}^{2}}^{2}\right] \\
& \quad+K h\left[\left\|\partial^{+} V\right\|_{\ell_{h}^{2}}+\left\|\partial^{+} \partial^{+} V\right\|_{\ell_{h}^{2}}\right] \\
\leq K \mathcal{E}_{\mathrm{nl}}(V), & \\
\left\|f_{\mathrm{nl} ; U}^{B}(V)\right\|_{\ell_{h}^{2}} \leq K h\left\|\partial^{+} V\right\|_{\ell_{h}^{2}} & \leq K \mathcal{E}_{\mathrm{nl}}(V)
\end{aligned}
$$

for all $U \in \Omega_{h ; \kappa}, h>0$ and $V \in \ell_{h}^{2}$.

Recalling the notation $g^{+}(U)=\partial^{+} g(U)$, the final set of nonlinearities is given by

$$
\mathcal{S}_{\mathrm{nl} ; V}=\left\{g, g^{+}\right\} \text {. }
$$

We write

$$
\begin{array}{ll}
g_{\text {apx }}(U)=g(U), & g_{\text {lin } ; U}[V]=g^{\prime}(U) V, \\
g_{\text {apx }}^{+}(U)=g^{\prime}(U) \partial^{0} U, & g_{\operatorname{lin} ; U}^{+}[V]=g^{\prime \prime}(U)\left[\partial^{0} U\right] V+g^{\prime}(U) \partial^{0} V .
\end{array}
$$

In addition, for every $f \in \mathcal{S}_{\mathrm{nl} ; V}$ we write

$$
Q_{f}=\{2, \infty\}, \quad Q_{f ; \operatorname{lin}}^{A}=Q_{f ; \operatorname{lin}}^{B}=\{2\}, \quad Q_{f ; \mathrm{nl}}^{A}=Q_{f ; \mathrm{nl}}^{B}=\{2\} .
$$

We recall that $Q_{g ; \text { pref }}=\bar{Q}_{g ; \text { pref }}=\{2\}$ and $\bar{Q}_{g^{+} ; \text {pref }}=\{2\}$.

Proposition 7.5 (see §F.6) Assume that $(\mathrm{Hg})$ is satisfied, fix $0<\kappa<\frac{1}{12}$ and pick any nonlinearity $f \in \mathcal{S}_{\mathrm{nl} ; V}$. Then there exists a constant $K>0$ so that (7.4) holds and the following properties are true.

(i) Upon introducing the seminorms

$$
\begin{aligned}
& {[V]_{f ; 2, h}=\|V\|_{\ell_{h}^{2}}+\left\|\partial^{+} V\right\|_{\ell_{h}^{2}} \leq \min \left\{S_{\text {full }}(V), S_{2 ; \text { fix }}(V)\right\},} \\
& {[V]_{f ; \infty, h}=\|V\|_{\ell_{h}^{\infty}}+\left\|\partial^{+} V\right\|_{\ell_{h}^{\infty}},}
\end{aligned}
$$

the conditions in $(h f)$ are all satisfied.

(ii) Upon writing $f_{\operatorname{lin} ; U}^{B}=0$, the conditions in $(h f)_{\operatorname{lin}}$ are satisfied. In addition, the bound

$$
\left\|f_{\operatorname{lin} ; U}^{A}[V]\right\|_{\ell_{h}^{2}} \leq K\left[\|V\|_{\ell_{h}^{2}}+\left\|\partial^{+} V\right\|_{\ell_{h}^{2}}\right] \leq K T_{\text {safe }}(V)
$$

holds for all $U \in \Omega_{h ; \kappa}, h>0$ and $V \in \ell_{h}^{2}$. 
(iii) Upon writing $f_{\mathrm{nl} ; U}^{B}=0$, the conditions in $(h f)_{\mathrm{nl}}$ are satisfied. In addition, we have the bound

$$
\begin{aligned}
\left\|f_{\mathrm{nl} ; U}^{A}(V)\right\|_{\ell_{h}^{2}} \leq & K\left[\|V\|_{\ell_{h}^{\infty}}+\left\|\partial^{+} V\right\|_{\ell_{h}^{\infty}}\right]\left[\|V\|_{\ell_{h}^{2}}+\left\|\partial^{+} V\right\|_{\ell_{h}^{2}}\right] \\
& K h\left[\|V\|_{\ell_{h}^{2}}+\left\|\partial^{+} V\right\|_{\ell_{h}^{2}}+\left\|\partial^{+} \partial^{+} V\right\|_{\ell_{h}^{2}}\right] \\
\leq & K \mathcal{E}_{\mathrm{nl}}(V)
\end{aligned}
$$

for all $U \in \Omega_{h ; \kappa}, h>0$ and $V \in \ell_{h}^{2}$.

\subsection{Bookkeeping}

Our task here is to interpret the raw estimates from $\$ 7.1$ and formulate some observations that will help us to apply Proposition 5.4 in a structured fashion. Our first result in combination with Proposition 6.4 ensures that the admissable sequences $q_{i, \operatorname{lin}}^{A}$ and $q_{i, \operatorname{lin}}^{B}$ appearing in (d) of Proposition 5.4 can always be sampled from the set of preferred exponents $Q_{f \text {;pref }}$. In principle, this allows us to only consider the contributions to the cross-term (5.49) that arise from these preferred exponents. However, the inclusion $\infty \in Q_{f}$ allows us to strategically depart from these exponents at times when evaluating (5.49) using the exponent-swapping procedure described in Lemma 5.3. Indeed, this requires one of the relevant exponents to be flipped to infinity.

Corollary 7.6 For every $f \in \mathcal{S}_{\mathrm{nl}}$ we have $\infty \in Q_{f}$ together with $Q_{f ; \text { pref }} \subset Q_{f} \cap Q_{f ; \text { in }}^{A} \cap$ $Q_{f ; \text { lin. }}^{B}$. The same properties hold upon replacing $\left(\mathcal{S}_{\mathrm{nl}}, Q_{f ; \mathrm{pref}}\right)$ by $\left(\overline{\mathcal{S}}_{\mathrm{nl}}, \bar{Q}_{f ; \mathrm{pref}}\right)$.

Proof The result can be verified by inspecting the definitions (6.18), (6.20), (7.9), (7.15), (7.23), (7.30) and (7.36).

The following two results formalize the interpretation of the terms $S_{2 \text {; fix }}$ and $T_{\infty ; \text { opt }}$ that we proposed at the start of this section. We emphasize that the (b) properties will be used to deviate from the preferred exponents strategically in order to prevent products of supremum bounds; see the proof of Lemma 8.1 below.

Corollary 7.7 Assume that $(\mathrm{Hg})$ is satisfied and fix $0<\kappa<\frac{1}{12}$. For every $f \in \mathcal{S}_{\mathrm{nl}}$ and $q \in Q_{f ; \text { pref }}$ we have

$$
[V]_{f ; q, h} \leq S_{\text {full }}(V)
$$

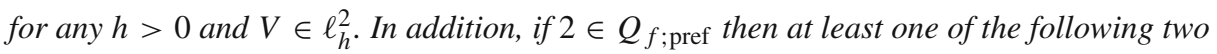
properties hold true.

(a) We have

$$
[V]_{f ; 2, h} \leq S_{2 ; \text { fix }}(V)
$$

for every $h>0$ and $V \in \ell_{h}^{2}$.

(b) We have

$$
[V]_{f ; \infty, h} \leq S_{\text {full }}(V)
$$

for every $h>0$ and $V \in \ell_{h}^{2}$.

The same properties hold upon replacing $\left(\mathcal{S}_{\mathrm{nl}}, Q_{f ; \text { pref }}, S_{\text {full }}, S_{2 ; \mathrm{fix}}\right)$ by $\left(\overline{\mathcal{S}}_{\mathrm{nl}}, \bar{Q}_{f ; \mathrm{pref}}, \bar{S}_{\text {full }}\right.$, $\bar{S}_{2 ; \text { fix }}$. 
Proof The result can be verified by inspecting the bounds in item (i) of Propositions 7.1-7.5.

Corollary 7.8 Assume that $(\mathrm{Hg})$ is satisfied and fix $0<\kappa<\frac{1}{12}$. For any $f \in \mathcal{S}_{\mathrm{nl}}$, any

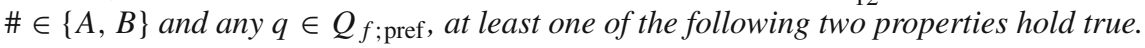

(a) There exists $K>0$ so that

$$
\left\|f_{\operatorname{lin} ; U}^{\#}[V]\right\|_{\ell_{h}^{q}} \leq K T_{\text {safe }}(V)
$$

holds for every $h>0, U \in \Omega_{h ; \kappa}$ and $V \in \ell_{h}^{2}$.

(b) We have $q=\infty$ and there exists $K>0$ so that the bounds

$$
\begin{aligned}
\left\|f_{\text {lin; } U}^{\#}[V]\right\|_{\ell_{h}^{2}} & \leq K T_{\text {safe }}(V), \\
\left\|f_{\text {lin; } U}^{\#}[V]\right\|_{\ell_{h}^{\infty}} & \leq K T_{\infty ; \text { opt }}(V)
\end{aligned}
$$

hold for every $h>0, U \in \Omega_{h ; \kappa}$ and $V \in \ell_{h}^{2}$.

The same properties hold upon replacing $\left(\mathcal{S}_{\mathrm{nl}}, Q_{f ; \mathrm{pref}}, T_{\mathrm{safe}}, T_{\infty ; \mathrm{opt}}\right)$ by $\left(\overline{\mathcal{S}}_{\mathrm{nl}}, \bar{Q}_{f ; \mathrm{pref}}, \bar{T}_{\mathrm{safe}}\right.$, $\left.\bar{T}_{\infty ; \text { opt }}\right)$.

Proof The result can be verified by inspecting the bounds in item (ii) of Propositions 7.1-7.5.

Our final result will be used to control the nonlinear contributions described in (5.48). The main point here is that at least one of the two possible norms on $f_{\mathrm{nl}, U}^{\#}$ will lead to the desired bound $\mathcal{E}_{\mathrm{nl}}(V)$ and that this can be arranged by exponent-swapping if necessary. The full details can again be found in the proof of Lemma 8.1 below.

Corollary 7.9 Assume that $(\mathrm{Hg})$ is satisfied and fix $0<\kappa<\frac{1}{12}$. Consider any $f \in \mathcal{S}_{\mathrm{nl}}$ and

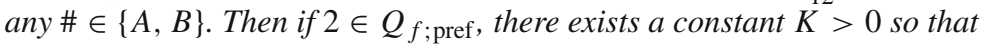

$$
\left\|f_{\mathrm{nl} ; U}^{\#}(V)\right\|_{\ell_{h}^{2}} \leq K \mathcal{E}_{\mathrm{nl}}(V)
$$

holds for all $h>0, U \in \Omega_{h ; \kappa}$ and $V \in \ell_{h}^{2}$ for which $U+V \in \Omega_{h ; \kappa}$.

Otherwise, there exists $q \in\{2, \infty\}$ together with a constant $K>0$ so that

$$
\left\|f_{\mathrm{nl} ; U}^{\#}(V)\right\|_{\ell_{h}^{q}} \leq K \mathcal{E}_{\mathrm{nl}}(V)
$$

holds for all $h>0, U \in \Omega_{h ; \kappa}$ and $V \in \ell_{h}^{2}$ for which $U+V \in \Omega_{h ; \kappa}$. The same properties hold upon replacing $\left(\mathcal{S}_{\mathrm{nl}}, Q_{f ; \mathrm{pref}}, \mathcal{E}_{\mathrm{nl}}\right)$ by $\left(\overline{\mathcal{S}}_{\mathrm{nl}}, \bar{Q}_{f ; \mathrm{pref}}, \overline{\mathcal{E}}_{\mathrm{nl}}\right)$.

Proof The result can be verified by inspecting the bounds in item (iii) of Propositions 7.1-7.5.

\section{Approximations for $\mathcal{G}$ and $\mathcal{G}^{+}$}

In this section we set out to apply Proposition 5.4 to the decompositions (6.10) and (6.16) for $\mathcal{G}(U)$ respectively $\mathcal{G}^{+}(U)$, using the approximate expressions introduced in $\S 7.1$. The estimates here are more than sufficient to establish our well-posedness result Proposition 2.4. Their primary purpose will become clear in the sequel paper [32], where our approximants 
will be simplified even further in order to construct travelling wave solutions to our adaptive grid problem. As we explain in the sequel, the main distinction is that we focus on nonlinear issues here, leaving a further analysis of the linear terms to [32].

Exploiting the definitions (6.7) and (6.11), we define the standard approximants

$$
\begin{aligned}
\mathcal{Y}_{1 ; \mathrm{apx}}(U) & =\mathcal{D}_{\mathrm{apx}}^{\diamond_{0}}(U) \mathcal{Z}_{\mathrm{apx}}^{-}(U), \\
\mathcal{Y}_{2 ; \mathrm{apx}}(U) & =\mathcal{D}_{\mathrm{apx}}^{\diamond_{0}}(U)+g(U), \\
\mathcal{Y}_{1 ; \mathrm{apx}}^{+}(U) & =\widetilde{p}_{\text {apx }}(U) \mathcal{D}_{\mathrm{apx}}^{\diamond_{0} ;+}(U) T^{+}\left[\mathcal{Z}_{\mathrm{apx}}^{-}(U)\right], \\
\mathcal{Y}_{2 a ; \mathrm{apx}}^{+}(U) & =\mathcal{D}_{a ; \mathrm{apx}}^{\diamond_{0} ;+}(U), \\
\mathcal{Y}_{2 b ; \mathrm{apx}}^{+}(U) & =\mathcal{D}_{b ; \mathrm{apx}}^{\diamond_{0} ;+}(U)+g_{\mathrm{apx}}^{+}(U),
\end{aligned}
$$

together with

$$
\begin{aligned}
\mathcal{Y}_{1 ; \operatorname{lin} ; U}[V]= & \mathcal{D}_{\operatorname{lin} ; U}^{\diamond_{0}}[V] \mathcal{Z}_{\mathrm{apx}}^{-}(U)+\mathcal{D}_{\mathrm{apx}}^{\diamond_{0}}(U) \mathcal{Z}_{\text {lin } ; U}^{-}[V], \\
\mathcal{Y}_{2 ; \operatorname{lin} ; U}[V]= & \mathcal{D}_{\operatorname{lin} ; U}^{\diamond_{0}}[V]+g^{\prime}(U) V, \\
\mathcal{Y}_{1 ; \operatorname{lin} ; U}^{+}[V]= & \tilde{p}_{\operatorname{lin} ; U}[V] \mathcal{D}_{\mathrm{apx}}^{\diamond_{0} ;+}(U) T^{+}\left[\mathcal{Z}_{\mathrm{apx}}^{-}(U)\right]+\tilde{p}_{\mathrm{apx}}(U) \mathcal{D}_{\operatorname{lin} ; U}^{\diamond_{0} ;+}[V] T^{+}\left[\mathcal{Z}_{\mathrm{apx}}^{-}(U)\right] \\
& +\widetilde{p}_{\mathrm{apx}}(U) \mathcal{D}_{\mathrm{apx}}^{\diamond_{0} ;+}(U) T^{+}\left[\mathcal{Z}_{\operatorname{lin} ; U}^{-}[V]\right], \\
\mathcal{Y}_{2 a ; \operatorname{lin} ; U}^{+}[V]= & \mathcal{D}_{a ; \operatorname{lin} ; U}^{\diamond_{0} ;+}[V], \\
\mathcal{Y}_{2 b ; \operatorname{lin} ; U}^{+}[V]= & \mathcal{D}_{b ; \operatorname{lin} ; U}^{\diamond_{0} ;+}[V]+g_{\operatorname{lin} ; U}^{+}[V] .
\end{aligned}
$$

In addition, exploiting the definitions (6.8), we write

$$
\begin{aligned}
& \mathcal{X}_{A ; \text { apx }}(U)=p_{\text {apx }}(U) \mathcal{Z}_{\text {apx }}^{+}(U), \\
& \mathcal{X}_{B ; \text { apx }}(U)=S^{+}\left[\mathcal{Z}_{\text {apx }}^{+}(U)\right] p_{\text {apx }}^{\diamond_{+}}(U), \\
& \mathcal{X}_{C ; \text { apx }}(U)=S^{+}\left[\mathcal{Z}_{\text {apx }}^{+}(U)\right] p_{\text {apx }}^{\diamond_{0}}(U), \\
& \mathcal{X}_{D ; \text { apx }}(U)=S^{+}\left[p_{\text {apx }}(U)\right] \mathcal{Z}_{\text {apx }}^{+}(U) p_{\text {apx }}(U),
\end{aligned}
$$

together with

$$
\begin{aligned}
\mathcal{X}_{A ; \operatorname{lin} ; U}[V]= & p_{\operatorname{lin} ; U}[V] \mathcal{Z}_{\text {apx }}^{+}(U)+p_{\text {apx }}(U) \mathcal{Z}_{\operatorname{lin} ; U}^{+}[V], \\
\mathcal{X}_{B ; \operatorname{lin} ; U}[V]= & S^{+}\left[\mathcal{Z}_{\operatorname{lin} ; U}^{+}[V]\right] p_{\text {apx }}^{\diamond_{+}}(U)+S^{+}\left[\mathcal{Z}_{\text {apx }}^{+}(U)\right] p_{\operatorname{lin} ; U}^{\diamond_{+}}[V], \\
\mathcal{X}_{C ; \operatorname{lin} ; U}[V]= & S^{+}\left[\mathcal{Z}_{\operatorname{lin} ; U}^{+}[V]\right] p_{\text {apx }}^{\diamond_{0}}(U)+S^{+}\left[\mathcal{Z}_{\text {apx }}^{+}(U)\right] p_{\operatorname{lin} ; U}^{\diamond_{0}}[V], \\
\mathcal{X}_{D ; \operatorname{lin} ; U}[V]= & S^{+}\left[p_{\operatorname{lin} ; U}[V]\right] \mathcal{Z}_{\text {apx }}^{+}(U) p_{\text {apx }}(U)+S^{+}\left[p_{\text {apx }}(U)\right] \mathcal{Z}_{\operatorname{lin} ; U}^{+}[V] p_{\text {apx }}(U) \\
& +S^{+}\left[p_{\text {apx }}(U)\right] \mathcal{Z}_{\text {apx }}^{+}(U) p_{\operatorname{lin} ; U}[V] .
\end{aligned}
$$

Using the expressions introduced in $\$ 7.1$ all these approximants can in principle be explicitly evaluated, although we refrain from doing this in the present paper. They can be used as building blocks for the expressions $P_{\text {apx }}$ and $P_{\text {lin; } U}$ defined in (5.44) that arise when applying Proposition 5.4 to $\mathcal{G}$ and $\mathcal{G}^{+}$.

\subsection{Estimates for $\mathcal{G}$}

Applying the expressions (5.44) to the terms (6.9), we obtain the initial expressions

$$
\mathcal{G}_{A ; \mathrm{apx} ; I}(U)=\left[1-\mathcal{Y}_{1 ; \mathrm{apx}}(U) T^{-}\left[\mathcal{X}_{A ; \mathrm{apx}}(U)\right]\right] \mathcal{Y}_{2 ; \mathrm{apx}}(U)
$$




$$
\begin{aligned}
& \mathcal{G}_{B ; \mathrm{apx} ; I}(U)=\mathcal{Y}_{1 ; \mathrm{apx}}(U) \sum_{-; h} \mathcal{Y}_{2 ; \mathrm{apx}}(U) T^{-}\left[\mathcal{X}_{B ; \mathrm{apx}}(U)\right] \mathcal{D}_{\mathrm{apx}}^{\diamond ;+}(U), \\
& \mathcal{G}_{C ; \mathrm{apx} ; I}(U)=\mathcal{Y}_{1 ; \mathrm{apx}}(U) \sum_{-; h} \mathcal{Y}_{2 ; \mathrm{apx}}(U) T^{-}\left[\mathcal{X}_{C ; \mathrm{apx}}(U) \mathcal{D}_{\mathrm{apx}}^{\diamond_{0} ;+}(U)\right], \\
& \mathcal{G}_{D ; \mathrm{apx} ; I}(U)=\mathcal{Y}_{1 ; \mathrm{apx}}(U) \sum_{-; h} \mathcal{Y}_{2 ; \mathrm{apx}}(U) T^{-}\left[\mathcal{X}_{D ; \mathrm{apx}}(U) \mathcal{D}_{\mathrm{apx}}^{\diamond_{0} ;+}(U)\right],
\end{aligned}
$$

together with

$$
\begin{aligned}
\mathcal{G}_{A ; \operatorname{lin} ; U ; I}[V]= & -\mathcal{Y}_{1 ; \operatorname{lin} ; U}[V] T^{-}\left[\mathcal{X}_{A ; \mathrm{apx}}(U)\right] \mathcal{Y}_{2 ; \mathrm{apx}}(U) \\
& -\mathcal{Y}_{1 ; \mathrm{apx}}(U) T^{-}\left[\mathcal{X}_{A ; \operatorname{lin} ; U}[V]\right] \mathcal{Y}_{2 ; \mathrm{apx}}(U) \\
& +\left[1-\mathcal{Y}_{1 ; \mathrm{apx}}(U) T^{-}\left[\mathcal{X}_{A ; \mathrm{apx}}(U)\right]\right] \mathcal{Y}_{2 ; \operatorname{lin} ; U}[V], \\
\mathcal{G}_{B ; \operatorname{lin} ; U ; I}[V]= & \mathcal{Y}_{1 ; \operatorname{lin} ; U}[V] \sum_{-; h} \mathcal{Y}_{2 ; \mathrm{apx}}(U) T^{-}\left[\mathcal{X}_{B ; \mathrm{apx}}(U)\right] \mathcal{D}_{\mathrm{apx}}^{\diamond_{-} ;}(U) \\
& +\mathcal{Y}_{1 ; \mathrm{apx}}(U) \sum_{-; h} \mathcal{Y}_{2 ; \operatorname{lin} ; U}[V] T^{-}\left[\mathcal{X}_{B ; \mathrm{apx}}(U)\right] \mathcal{D}_{\mathrm{apx}}^{\diamond ;+}(U) \\
& +\mathcal{Y}_{1 ; \mathrm{apx}}(U) \sum_{-; h} \mathcal{Y}_{2 ; \mathrm{apx}}(U) T^{-}\left[\mathcal{X}_{B ; \operatorname{lin} ; U}[V]\right] \mathcal{D}_{\mathrm{apx}}^{\diamond_{-} ;+}(U) \\
& +\mathcal{Y}_{1 ; \mathrm{apx}}(U) \sum_{-; h} \mathcal{Y}_{2 ; \mathrm{apx}}(U) T^{-}\left[\mathcal{X}_{B ; \mathrm{apx}}(U)\right] \mathcal{D}_{\text {lin; } ;}^{\diamond ;+}[V]
\end{aligned}
$$

and finally

$$
\begin{aligned}
\mathcal{G}_{\# ; \operatorname{lin} ; U ; I}[V]= & \mathcal{Y}_{1 ; \operatorname{lin} ; U}[V] \sum_{-; h} \mathcal{Y}_{2 ; \mathrm{apx}}(U) T^{-}\left[\mathcal{X}_{\# ; \mathrm{apx}}(U) \mathcal{D}_{\mathrm{apx}}^{\diamond_{0} ;+}(U)\right] \\
& +\mathcal{Y}_{1 ; \mathrm{apx}}(U) \sum_{-; h} \mathcal{Y}_{2 ; \operatorname{lin} ; U}[V] T^{-}\left[\mathcal{X}_{\# ; \mathrm{apx}}(U) \mathcal{D}_{\mathrm{apx}}^{\diamond_{0} ;+}(U)\right] \\
& +\mathcal{Y}_{1 ; \mathrm{apx}}(U) \sum_{-; h} \mathcal{Y}_{2 ; \mathrm{apx}}(U) T^{-}\left[\mathcal{X}_{\# ; \operatorname{lin} ; U}[V] \mathcal{D}_{\mathrm{apx}}^{\diamond_{0} ;+}(U)\right] \\
& +\mathcal{Y}_{1 ; \mathrm{apx}}(U) \sum_{-; h} \mathcal{Y}_{2 ; \mathrm{apx}}(U) T^{-}\left[\mathcal{X}_{\# ; \mathrm{apx}}(U) \mathcal{D}_{\operatorname{lin} ; U}^{\diamond_{0} ;+}[V]\right]
\end{aligned}
$$

for \# $\in\{C, D\}$. Combining these expressions, we introduce the initial approximants

$$
\begin{aligned}
\mathcal{G}_{\mathrm{apx} ; I}(U) & =\mathcal{G}_{A ; \operatorname{apx} ; I}(U)+\mathcal{G}_{B ; \mathrm{apx} ; I}(U)+\mathcal{G}_{C ; \mathrm{apx} ; I}(U)+\mathcal{G}_{D ; \mathrm{apx} ; I}(U), \\
\mathcal{G}_{\operatorname{lin} ; U ; I}[V] & =\mathcal{G}_{A ; \operatorname{lin} ; U ; I}[V]+\mathcal{G}_{B ; \operatorname{lin} ; U ; I}[V]+\mathcal{G}_{C ; \operatorname{lin} ; U ; I}[V]+\mathcal{G}_{D ; \operatorname{lin} ; U ; I}[V]
\end{aligned}
$$

and write

$$
\mathcal{G}_{\mathrm{nl} ; U ; I}(V)=\mathcal{G}(U+V)-\mathcal{G}(U)-\mathcal{G}_{\operatorname{lin} ; U ; I}[V] .
$$

These expressions are sufficient for our purposes here, but will be expanded and simplified further in [32]. In particular, we obtain a crucial bound on the nonlinear residual here, which is then improved further in [32] by manipulating the linear terms $\mathcal{G}_{\operatorname{lin} ; U ; I}$. We recall that the higher-order sequence spaces $\ell_{h}^{2 ; 2}$ and $\ell_{h}^{\infty ; 1}$ were defined in (3.34) and (3.35). 
Lemma 8.1 Suppose that $(\mathrm{Hg})$ is satisfied and fix $0<\kappa<\frac{1}{12}$. Then there exists $K>0$ so that the approximation estimate

$$
\left\|\mathcal{G}(U)-\mathcal{G}_{\text {apx } ; I}(U)\right\|_{\ell_{h}^{2}} \leq K h
$$

and the residual bound

$$
\left\|\mathcal{G}_{\mathrm{nl} ; U ; I}(V)\right\|_{\ell_{h}^{2}} \leq K\|V\|_{\ell_{h}^{2 ; 2}}\left[\|V\|_{\ell_{h}^{2 ; 2}}+\|V\|_{\ell_{h}^{\infty ; 1}}+h\right]
$$

both hold for any $h>0$, any $U \in \Omega_{h ; \kappa}$ and any $V \in \ell_{h}^{2}$ for which $U+V \in \Omega_{h ; \kappa}$.

Proof Our strategy is to apply Proposition 5.4 to each of the products in the decomposition of $\mathcal{G}(U)$ obtained in Corollary 6.4. Let us therefore consider a single element of the sum (6.27), which we characterize by the set $\left(\pi, \mathbf{q}_{\pi}, \mathbf{f}, k\right)$.

Recalling (7.3), we first claim that

$$
\mathcal{J}_{\mathrm{nl} ; U}(V) \leq C_{1}^{\prime} \mathcal{E}_{\mathrm{nl}}(V) .
$$

Indeed, consider any $1 \leq i \leq k$ and any $\# \in\{A, B\}$. If $q_{\pi ; i}=2$, then certainly $2 \in Q_{f_{i}}$; pref by item (iii) of Corollary 6.4 , which allows us to take

$$
\mathbf{q}_{i, \mathrm{nl}}^{\#}=\mathbf{q}_{\pi}
$$

for the sequences in item (c) of Proposition 5.4. This allows us to apply (7.45), as desired. Suppose therefore that $q_{\pi ; i}=\infty$ and consider the integer $q$ defined in Corollary 7.9. If $q=\infty$, then we can again take $\mathbf{q}_{i, \mathrm{nl}}^{\#}=\mathbf{q}_{\pi}$ and apply (7.46). If $q=2$, then we choose $\mathbf{q}_{i, \mathrm{nl}}^{\#}$ to be the admissable sequence defined by the swapping Lemma 5.3, which has

$$
\mathbf{q}_{i, \mathrm{nl} ; i}^{\#}=2, \quad \mathbf{q}_{i, \mathrm{nl} ; j_{*}[i]}^{\#}=\infty .
$$

Corollary 7.6 shows that $\infty \in Q_{f_{j_{*}[i]}}$, which now again allows us to apply (7.46).

Our second claim is that

$$
\begin{aligned}
\mathcal{J}_{\text {cross } ;}(V) & \leq C_{2}^{\prime}\left[T_{\text {safe }}(V) S_{\text {full }}(V)+T_{\infty ; \text { opt }}(V) S_{2 ; \text { fix }}(V)\right] \\
& \leq C_{3}^{\prime} \mathcal{E}_{\text {nl }}(V) .
\end{aligned}
$$

Indeed, consider any \# $\in\{A, B\}$ and any pair $(i, j) \in\{1, \ldots, k\}^{2}$ with $i \neq j$. If item (a) in Corollary 7.8 holds for $f_{i}$ and $q=q_{\pi ; i}$, then the claim follows from (7.40). Suppose therefore that item (b) in Corollary 7.8 holds for $f_{i}$ and $q=q_{\pi ; i}=\infty$.

Write $\mathbf{q}_{\mathrm{sw}}$ for the admissable sequence defined by the swapping Lemma 5.3. If $j_{*}[i] \neq j$, then we have $q_{\mathrm{sw} ; j}=q_{\pi ; j}$. Writing $\mathbf{q}_{i j, \text { lin }}^{\#}=\mathbf{q}_{\mathrm{sw}}$ for the sequence in item (d) of Proposition 5.4 , the contribution from the pair $(i, j)$ can be absorbed by $T_{\text {safe }}(V) S_{\text {full }}(V)$. On the other hand, if $j_{*}[i]=j$, then $q_{\mathrm{sw} ; j}=\infty$ and $q_{\pi ; j}=2$. If item (b) of Corollary 7.7 holds, then we again write $\mathbf{q}_{i j, \text { lin }}^{\#}=\mathbf{q}_{\mathrm{sw}}$, noting that the contribution can be bounded by $T_{\text {safe }}(V) S_{\text {full }}(V)$. However, we write $\mathbf{q}_{i j, \text { lin }}^{\#}=\mathbf{q}_{\pi}$ if item (a) of Corollary 7.7 holds. In this case the contribution from the pair $(i, j)$ can be bounded by $T_{\infty ; \text { opt }}(V) S_{2 ; \text { fix }}(V)$.

Our final claim is that

$$
\mathcal{J}_{\text {apx } ; U}(V) \leq C_{4}^{\prime} h T_{\text {safe }}(V)=C_{4}^{\prime} h\|V\|_{\ell_{h}^{2 ; 2}} .
$$

This follows directly from the fact that $\left\|f(U)-f_{\text {apx }}(U)\right\|_{\ell_{h}^{q}} \leq K h$ for every $f \in \mathcal{S}_{\text {nl }}$ and $q \in Q_{f}$, together with the swapping technique described above. We note that this observation also implies the bound (8.10). 
Proof of Proposition 2.4 On account of Proposition 3.2, it is possible to pick constants $0<$ $\kappa<\frac{1}{12}$ and $\epsilon_{0}$ such that for any

$$
V \in \tilde{\mathcal{V}}_{\epsilon_{0}}:=\left\{V \in \ell_{h}^{2}:\|V\|_{\ell_{h}^{2}}<\epsilon_{0}\right\},
$$

we have $U_{0}+V \in \Omega_{h ; \kappa}$.

Recalling the continuous embedding $\ell_{h}^{2} \subset \ell_{h}^{\infty}$ and inspecting the explicit expressions in $\S 7$, the identity

$$
\begin{aligned}
\mathcal{G}\left(U_{0}+V^{(2)}\right)-\mathcal{G}\left(U_{0}+V^{(1)}\right) & =\mathcal{G}\left(U_{0}+V^{(1)}+\left(V^{(2)}-V^{(1)}\right)\right)-\mathcal{G}\left(U_{0}+V^{(1)}\right) \\
& =\mathcal{G}_{\operatorname{lin} ; U_{0}+V^{(1)} ; I}\left[V^{(2)}-V^{(1)}\right]+\mathcal{G}_{\mathrm{nl} ; U_{0}+V^{(1)} ; I}\left(V^{(2)}-V^{(1)}\right)
\end{aligned}
$$

together with the estimate (8.11) imply that the map

$$
V \mapsto \mathcal{G}\left(U_{0}+V\right) \in \ell_{h}^{2}
$$

is Lipschitz smooth on the set $\widetilde{V}_{\epsilon_{0}}$. The result now follows from standard ODE theory.

\subsection{Estimates for $\mathcal{G}^{+}$.}

Although results concerning $\mathcal{G}^{+}$were not needed to establish Proposition 2.4, we conclude this paper by applying the expressions (5.44) to this discrete derivative, in preparation for the sequel paper [32]. Starting with the term

$$
\mathcal{G}_{A^{\prime} a}^{+}(U)=\widetilde{p}(U) \mathcal{I}_{+}^{\diamond \diamond_{0} ;+}(U) \partial^{+} \partial^{(2)} U
$$

defined in (6.23), we obtain the initial approximants

$$
\begin{aligned}
\mathcal{G}_{A^{\prime} a ; \mathrm{apx} ; I}^{+}(U)= & \tilde{p}_{\text {apx }}(U) \mathcal{I}_{+; \text {apx }}^{\diamond_{0} ;+}(U) \partial^{+} \partial^{(2)} U, \\
\mathcal{G}_{A^{\prime} a ; \operatorname{lin} ; U ; I}^{+}[V]= & \widetilde{p}_{\operatorname{lin} ; U}[V] \mathcal{I}_{+; \text {apx }}^{\diamond \diamond_{0} ;+}(U) \partial^{+} \partial^{(2)} U+\widetilde{p}_{\text {apx }}(U) \mathcal{I}_{+; \operatorname{lin} ; U}^{\diamond_{0} ;+}[V] \partial^{+} \partial^{(2)} U \\
& +\widetilde{p}_{\text {apx }}(U) \mathcal{I}_{+; \text {apx }}^{\diamond \diamond_{0} ;+}(U) \partial^{+} \partial^{(2)} V
\end{aligned}
$$

and write

$$
\mathcal{G}_{A^{\prime} a ; \mathrm{nl} ; U ; I}^{+}(V)=\mathcal{G}_{A^{\prime} a}^{+}(U+V)-\mathcal{G}_{A^{\prime} a}^{+}(U)-\mathcal{G}_{A^{\prime} a ; \operatorname{lin} ; U ; I}^{+}[V] .
$$

It is essential to carefully track the appearance of third differences, which we achieve in the bounds below.

Lemma 8.2 Suppose that $(\mathrm{Hg})$ is satisfied and fix $0<\kappa<\frac{1}{12}$. Then there exists $K>0$ so that the approximation estimate

$$
\left\|\mathcal{G}_{A^{\prime} a}^{+}(U)-\mathcal{G}_{A^{\prime} a ; a p x ; I}^{+}(U)\right\|_{\ell_{h}^{2}} \leq K h\left\|\partial^{+} \partial^{+} \partial^{+} U\right\|_{\ell_{h}^{2}}
$$

and the residual bound

$$
\begin{aligned}
\left\|\mathcal{G}_{A^{\prime} a ; \mathrm{nl} ; U ; I}^{+}(V)\right\|_{\ell_{h}^{2}} \leq & K\left[\left\|\partial^{+} V\right\|_{\ell_{h}^{\infty}}+h\right]\left\|\partial^{+} \partial^{+} \partial^{+} V\right\|_{\ell_{h}^{2}} \\
& +K\left\|\partial^{+} V\right\|_{\ell_{h}^{\infty}}\left\|\partial^{+} V\right\|_{\ell_{h}^{2}}\left\|\partial^{+} \partial^{+} \partial^{+} U\right\|_{\ell_{h}^{\infty}} \\
& +K h\left[\left\|\partial^{+} V\right\|_{\ell_{h}^{2}}+\left\|\partial^{+} \partial^{+} V\right\|_{\ell_{h}^{2}}\right]\left\|\partial^{+} \partial^{+} \partial^{+} U\right\|_{\ell_{h}^{\infty}}
\end{aligned}
$$

both hold for any $h>0$, any $U \in \Omega_{h ; \kappa}$ and any $V \in \ell_{h}^{2}$ for which $U+V \in \Omega_{h ; \kappa}$. 
Proof The first estimate follows immediately from Proposition 7.2. To obtain the second estimate, we observe that the uniform bound in item (i) of this proposition shows that

$$
\begin{aligned}
\left\|\mathcal{G}_{A^{\prime} a ; \mathrm{nl} ; U ; I}^{+}(V)\right\|_{\ell_{h}^{2}} \leq & C_{1}^{\prime}\left\|\partial^{+} \partial^{+} \partial^{+} V\right\|_{\ell_{h}^{2}}\left[\left\|\tilde{p}_{\operatorname{lin} ; U}[V]\right\|_{\ell_{h}^{\infty}}+\left\|\tilde{p}_{\mathrm{nl} ; U}(V)\right\|_{\ell_{h}^{\infty}}\right. \\
& \left.+\left\|\mathcal{I}_{+; \operatorname{lin} ; U}^{\diamond_{0} ;+}[V]\right\|_{\ell_{h}^{\infty}}+\left\|\mathcal{I}_{+; \otimes_{0} ;+}(V)\right\|_{\ell_{h}^{\infty}}\right] \\
& +C_{1}^{\prime}\left\|\partial^{+} \partial^{+} \partial^{+} U\right\|_{\ell_{h}^{\infty}}\left[\left\|\tilde{p}_{\mathrm{nl} ; U}(V)\right\|_{\ell_{h}^{2}}+\left\|\mathcal{I}_{+; \mathrm{nl} ; U}^{\diamond \diamond_{0} ;+}(V)\right\|_{\ell_{h}^{2}}\right. \\
& \left.+\left\|\widetilde{p}_{\operatorname{lin} ; U}[V]\right\|_{\ell_{h}^{2}}\left\|\mathcal{I}_{+; \operatorname{lin} ; U}^{\diamond \diamond_{0} ;+}[V]\right\|_{\ell_{h}^{\infty}}\right] .
\end{aligned}
$$

We note that Lemma's F.6 and F.8 yield the preliminary estimates

$$
\begin{aligned}
\left\|\tilde{p}_{\mathrm{nl} ; U}(V)\right\|_{\ell_{h}^{\infty}}+\left\|\mathcal{I}_{+; \text {nl } ; U}^{\diamond \diamond_{0} ;+}(V)\right\|_{\ell_{h}^{\infty}} & \leq C_{2}^{\prime}\left\|\partial^{+} V\right\|_{\ell_{h}^{\infty}}^{2}+C_{2}^{\prime} h\left[\left\|\partial^{+} V\right\|_{\ell_{h}^{\infty}}^{2}+\left\|\partial^{+} \partial^{+} V\right\|_{\ell_{h}^{\infty}}\right] \\
& \leq C_{3}^{\prime}\left[\left\|\partial^{+} V\right\|_{\ell_{h}^{\infty}}+h\right] .
\end{aligned}
$$

In addition, Proposition 7.2 yields the bounds

$$
\begin{aligned}
\left\|\tilde{p}_{\operatorname{lin} ; U}[V]\right\|_{\ell_{h}^{2}} & \leq C_{4}^{\prime}\left\|\partial^{+} V\right\|_{\ell_{h}^{2}}, \\
\left\|\tilde{p}_{\text {lin; } ;}[V]\right\|_{\ell_{h}^{\infty}}+\left\|\mathcal{I}_{+; \operatorname{lin} ; U}^{\diamond \diamond_{0} ;+}[V]\right\|_{\ell_{h}^{\infty}} & \leq C_{4}^{\prime}\left\|\partial^{+} V\right\|_{\ell_{h}^{\infty}},
\end{aligned}
$$

together with

$$
\begin{aligned}
& \left\|\tilde{p}_{\mathrm{nl} ; U}(V)\right\|_{\ell_{h}^{2}}+\left\|\mathcal{I}_{+; \mathrm{nl} ; U}^{\diamond \diamond_{0} ;+}(V)\right\|_{\ell_{h}^{2}} \\
& \leq C_{6}^{\prime}\left\|\partial^{+} V\right\|_{\ell_{h}^{\infty}}\left\|\partial^{+} V\right\|_{\ell_{h}^{2}}+C_{6}^{\prime} h\left[\left\|\partial^{+} V\right\|_{\ell_{h}^{2}}+\left\|\partial^{+} \partial^{+} V\right\|_{\ell_{h}^{2}}\right] .
\end{aligned}
$$

Substituting these bounds into (8.25) yields the desired estimate.

We now apply (5.44) to the terms (6.14) to obtain the initial approximants

$$
\begin{aligned}
& \mathcal{G}_{A^{\prime} b ; \mathrm{apx} ; I}^{+}(U)=\left[1-\mathcal{Y}_{1 ; \mathrm{apx}}(U) \mathcal{X}_{A ; \mathrm{apx}}(U)\right] \mathcal{Y}_{2 b ; \mathrm{apx}}^{+}(U), \\
& \mathcal{G}_{A^{\prime} c ; \mathrm{apx} ; I}^{+}(U)=-\mathcal{Y}_{1 ; \mathrm{apx}}^{+}(U) \mathcal{X}_{A ; \mathrm{apx}}(U) T^{+}\left[\mathcal{Y}_{2 ; \mathrm{apx}}(U)\right],
\end{aligned}
$$

together with

$$
\begin{aligned}
\mathcal{G}_{A^{\prime} b ; \operatorname{lin} ; U ; I}^{+}[V]= & \left.-\mathcal{Y}_{1 ; \operatorname{lin} ; U}[V] \mathcal{X}_{A ; \mathrm{apx}}(U) \mathcal{Y}_{2 b ; \mathrm{apx}}^{+}(U)\right] \\
& \left.-\mathcal{Y}_{1 ; \mathrm{apx}}(U) \mathcal{X}_{A ; \operatorname{lin} ; U}[V] \mathcal{Y}_{2 b ; \mathrm{apx}}^{+}(U)\right] \\
& {\left.\left[1-\mathcal{Y}_{1 ; \mathrm{apx}}(U) \mathcal{X}_{A ; \mathrm{apx}}(U)\right] \mathcal{Y}_{2 b ; \operatorname{lin} ; U}^{+}[V]\right], } \\
\mathcal{G}_{A^{\prime} c ; \operatorname{lin} ; U ; I}^{+}[V]= & -\mathcal{Y}_{1 ; \operatorname{lin} ; U}^{+}[V] \mathcal{X}_{A ; \mathrm{apx}}(U) T^{+}\left[\mathcal{Y}_{2 ; \mathrm{apx}}(U)\right] \\
& -\mathcal{Y}_{1 ; \mathrm{apx}}^{+}(U) \mathcal{X}_{A ; \operatorname{lin} ; U}[V] T^{+}\left[\mathcal{Y}_{2 ; \mathrm{apx}}(U)\right] \\
& -\mathcal{Y}_{1 ; \mathrm{apx}}^{+}(U) \mathcal{X}_{A ; \mathrm{apx}}(U) T^{+}\left[\mathcal{Y}_{2 ; \operatorname{lin} ; U}[V]\right] .
\end{aligned}
$$

Applying the expressions (5.44) one final time to the terms (6.15), we also obtain

$$
\left.\mathcal{G}_{B^{\prime} ; \mathrm{apx} ; I}^{+}(U)=\mathcal{Y}_{1 ; \mathrm{apx}}^{+}(U) T^{+} \sum_{-; h} \mathcal{Y}_{2 ; \mathrm{apx}}(U) T^{-}\left[\mathcal{X}_{B ; \mathrm{apx}}(U)\right] \mathcal{D}_{\mathrm{apx}}^{\diamond ;+}(U)\right],
$$




$$
\begin{aligned}
\mathcal{G}_{B^{\prime} ; \operatorname{lin} ; U ; I}^{+}[V]= & \mathcal{Y}_{1 ; \operatorname{lin} ; U}^{+}[V] T^{+} \sum_{-; h} \mathcal{Y}_{2 ; \text { apx }}(U) T^{-}\left[\mathcal{X}_{B ; \mathrm{apx}}(U)\right] \mathcal{D}_{\mathrm{apx}}^{\diamond_{-} ;+}(U) \\
& +\mathcal{Y}_{1 ; \text { apx }}^{+}(U) T^{+} \sum_{-; h} \mathcal{Y}_{2 ; \operatorname{lin} ; U}[V] T^{-}\left[\mathcal{X}_{B ; \mathrm{apx}}(U)\right] \mathcal{D}_{\mathrm{apx}}^{\diamond_{-} ;+}(U) \\
& +\mathcal{Y}_{1 ; \text { apx }}^{+}(U) T^{+} \sum_{-; h} \mathcal{Y}_{2 ; \mathrm{apx}}(U) T^{-}\left[\mathcal{X}_{B ; \operatorname{lin} ; U}[V]\right] \mathcal{D}_{\mathrm{apx}}^{\diamond_{-} ;+}(U) \\
& +\mathcal{Y}_{1 ; \text { apx }}^{+}(U) T^{+} \sum_{-; h} \mathcal{Y}_{2 ; \mathrm{apx}}(U) T^{-}\left[\mathcal{X}_{B ; \mathrm{apx}}(U)\right] \mathcal{D}_{\text {lin;U }}^{\diamond_{-} ;+}[V]
\end{aligned}
$$

together with

$$
\begin{aligned}
& \mathcal{G}_{\#^{\prime} ; \mathrm{apx} ; I}^{+}(U)=\mathcal{Y}_{1 ; \mathrm{apx}}^{+}(U) T^{+} \sum_{-; h} \mathcal{Y}_{2 ; \mathrm{apx}}(U) T^{-}\left[\mathcal{X}_{\# ; \mathrm{apx}}(U) \mathcal{D}_{\mathrm{apx}}^{\diamond_{0} ;+}(U)\right], \\
& \mathcal{G}_{\#^{\prime} ; \operatorname{lin} ; U ; I}^{+}[V]=\mathcal{Y}_{1 ; \operatorname{lin} ; U}^{+}[V] T^{+} \sum_{-; h} \mathcal{Y}_{2 ; \text { apx }}(U) T^{-}\left[\mathcal{X}_{\# ; \text { apx }}(U) \mathcal{D}_{\text {apx }}^{\diamond_{0} ;+}(U)\right] \\
& +\mathcal{Y}_{1 ; \mathrm{apx}}^{+}(U) T^{+} \sum_{-; h} \mathcal{Y}_{2 ; \operatorname{lin} ; U}[V] T^{-}\left[\mathcal{X}_{\# ; \mathrm{apx}}(U) \mathcal{D}_{\mathrm{apx}}^{\diamond_{0} ;+}(U)\right] \\
& +\mathcal{Y}_{1 ; \text { apx }}^{+}(U) T^{+} \sum_{-; h} \mathcal{Y}_{2 ; \text { apx }}(U) T^{-}\left[\mathcal{X}_{\# ; \operatorname{lin} ; U}[V] \mathcal{D}_{\text {apx }}^{\diamond_{0} ;+}(U)\right] \\
& +\mathcal{Y}_{1 ; \text { apx }}^{+}(U) T^{+} \sum_{-; h} \mathcal{Y}_{2 ; \text { apx }}(U) T^{-}\left[\mathcal{X}_{\# ; \text { apx }}(U) \mathcal{D}_{\text {lin } ; U}^{\diamond_{0} ;+}[V]\right]
\end{aligned}
$$

for \# $\in\{C, D\}$.

Writing

$$
\mathcal{G}_{\text {low }}^{+}(U)=\mathcal{G}^{+}(U)-\mathcal{G}_{A^{\prime} a}^{+}(U)
$$

we use the expressions above to introduce the initial approximants

$$
\begin{aligned}
\mathcal{G}_{\text {low;apx } ; I}^{+}(U)= & \mathcal{G}_{A^{\prime} b ; \text { apx } ; I}^{+}(U)+\mathcal{G}_{A^{\prime} c ; \text { apx } ; I}^{+}(U) \\
& +\mathcal{G}_{B^{\prime} ; \mathrm{apx} ; I}^{+}(U)+\mathcal{G}_{C^{\prime} ; \mathrm{apx} ; I}^{+}(U)+\mathcal{G}_{D^{\prime} ; \mathrm{apx} ; I}^{+}(U), \\
\mathcal{G}_{\text {low;lin; } ; ; I}^{+}[V]= & \mathcal{G}_{A^{\prime} b ; \operatorname{lin} ; U ; I}^{+}[V]+\mathcal{G}_{A^{\prime} c ; \operatorname{lin} ; U ; I}^{+}[V] \\
& +\mathcal{G}_{B^{\prime} ; \operatorname{lin} ; U ; I}^{+}[V]+\mathcal{G}_{C^{\prime} ; \operatorname{lin} ; U ; I}^{+}[V]+\mathcal{G}_{D^{\prime} ; \operatorname{lin} ; U ; I}^{+}[V]
\end{aligned}
$$

and write

$$
\mathcal{G}_{\text {low;nl } ; U ; I}^{+}(V)=\mathcal{G}_{\text {low }}^{+}(U+V)-\mathcal{G}_{\text {low }}^{+}(U)-\mathcal{G}_{\text {low;lin; } U ; I}^{+}[V] .
$$

Again, these expressions will be further analyzed and simplified in [32] using a different estimation procedure on the linear terms. For now, we apply Proposition 5.4 to obtain an initial estimate on the nonlinear residual, which will play an important role in the sequel [32].

Lemma 8.3 Suppose that $(\mathrm{Hg})$ is satisfied and fix $0<\kappa<\frac{1}{12}$. Then there exists $K>0$ so that the approximation estimate

$$
\left\|\mathcal{G}_{\text {low }}^{+}(U)-\mathcal{G}_{\text {low; apx; } I}^{+}(U)\right\|_{\ell_{h}^{2}} \leq K h
$$


and the residual bound

$$
\left\|\mathcal{G}_{\text {low;nl } ; I}^{+}(V)\right\|_{\ell_{h}^{2}} \leq K\|V\|_{\ell_{h}^{2 ; 2}}\left[\|V\|_{\ell_{h}^{2 ; 2}}+\|V\|_{\ell_{h}^{\infty ; 2}}+h\right]
$$

both hold for any $h>0$, any $U \in \Omega_{h ; \kappa}$ and any $V \in \ell_{h}^{2}$ for which $U+V \in \Omega_{h ; \kappa}$.

Proof Following the strategy developed in the proof of Lemma 8.1, the error terms in Proposition 5.4 can be controlled by

$$
\begin{aligned}
\mathcal{J}_{\mathrm{nl} ; U}(V) & \leq C_{1}^{\prime} \overline{\mathcal{E}}_{\mathrm{nl}}(V) \\
& \leq C_{2}^{\prime}\|V\|_{\ell_{h}^{2 ; 2}}\left[\|V\|_{\ell_{h}^{2 ; 2}}+\|V\|_{\ell_{h}^{\infty ; 2}}+h\right], \\
\mathcal{J}_{\text {cross } ; U}(V) & \leq C_{1}^{\prime}\left[\bar{T}_{\mathrm{safe}}(V) \bar{S}_{\mathrm{full}}(V)+\bar{T}_{\infty ; \mathrm{opt}}(V) \bar{S}_{2 ; \mathrm{fix}}(V)\right] \\
& \leq C_{2}^{\prime}\|V\|_{\ell_{h}^{2 ; 2}}\left[\|V\|_{\ell_{h}^{2 ; 2}}+\|V\|_{\ell_{h}^{\infty ; 2}}\right], \\
\mathcal{J}_{\text {apx } ; U}(V) & \leq C_{1}^{\prime} h \bar{T}_{\mathrm{safe}}(V) \\
& =C_{1}^{\prime} h\|V\|_{\ell_{h}^{2 ; 2}},
\end{aligned}
$$

which yields the desired bounds.

Acknowledgements Hupkes acknowledges support from the Netherlands Organization for Scientific Research (NWO) (Grant 639.032.612). Van Vleck acknowledges support from the NSF (DMS-1419047 and DMS1714195). Both authors wish to thank W. Huang for helpful discussions during the conception and writing of this paper. Both authors also wish to thank two anonymous referees for a very detailed reading of the paper resulting in many constructive remarks.

Open Access This article is licensed under a Creative Commons Attribution 4.0 International License, which permits use, sharing, adaptation, distribution and reproduction in any medium or format, as long as you give appropriate credit to the original author(s) and the source, provide a link to the Creative Commons licence, and indicate if changes were made. The images or other third party material in this article are included in the article's Creative Commons licence, unless indicated otherwise in a credit line to the material. If material is not included in the article's Creative Commons licence and your intended use is not permitted by statutory regulation or exceeds the permitted use, you will need to obtain permission directly from the copyright holder. To view a copy of this licence, visit http://creativecommons.org/licenses/by/4.0/.

\section{A Sequence Sampling}

In order to link classical continuum theory to the discrete setting of the adaptive grid, we often need to extract sequences from continuous functions and relate discrete derivatives to their continuous counterparts. To facilitate this, we obtain several useful results here that relate the $\ell_{h}^{q}$-norms of sequences $v(h \mathbb{Z}+\vartheta)$ sampled from a function $v$ back to $L^{q}$-norms of $v$ and its derivatives.

For any $f \in L^{2}$ and $h>0$, we formally write

$$
\left[\partial_{h}^{+} f\right](\tau)=h^{-1}[f(\tau+h)-f(\tau)], \quad\left[\partial_{h}^{-} f\right](\tau)=h^{-1}[f(\tau)-f(\tau-h)],(\text { A. } 1)
$$

which obviously satisfy $\partial_{h}^{ \pm} f \in L^{2}$. In addition, for any bounded continuous function $f$, any $\vartheta \in \mathbb{R}$ and any $h>0$, we write $\operatorname{ev}_{\vartheta} f \in \ell_{h}^{\infty}$ for the sequence

$$
\left[\operatorname{ev}_{\vartheta} f\right]_{j h}=f(\vartheta+j h)
$$

When the context is clear, we often simply write $f$ to refer to the sampled sequence $\operatorname{ev}_{0} f$. 
Lemma A.1 Pick $q \in\{2, \infty\}$ and consider any $u \in W^{1, q}$. Then the estimates

$$
\left\|\partial_{h}^{ \pm} u\right\|_{\ell_{h}^{q}} \leq\left\|u^{\prime}\right\|_{L^{q}}
$$

hold for any $h>0$. If $q=2$, then we also have

$$
\left\|\partial_{h}^{ \pm} u\right\|_{\ell_{h}^{\infty}} \leq h^{-1 / 2}\left\|u^{\prime}\right\|_{L^{2}}
$$

for all $h>0$.

Proof For $q=\infty$ the statement is immediate, so assume that $q=2$. We may then compute

$$
\begin{aligned}
\left\|\partial_{h}^{+} u\right\|_{\ell_{h}^{2}}^{2} & =h \sum_{j \in \mathbb{Z}} \frac{(u((j+1) h)-u(j h))^{2}}{h^{2}} \\
& =h \sum_{j \in \mathbb{Z}} h^{-2}\left[\int_{0}^{h} u^{\prime}(j h+s) d s\right]^{2} \\
& \leq h \sum_{j \in \mathbb{Z}} h^{-2} h \int_{0}^{h} u^{\prime}(j h+s)^{2} d s \\
& =\sum_{j \in \mathbb{Z}} \int_{0}^{h} u^{\prime}(j h+s)^{2} d s \\
& =\left\|u^{\prime}\right\|_{L^{2}}^{2} .
\end{aligned}
$$

In addition, the identity (A.4) follows directly from (3.33).

Lemma A.2 For any $u \in H^{1}$ and any $h>0$ we have

$$
\|u\|_{\ell_{h}^{2}} \leq(2+h)\|u\|_{H^{1}} .
$$

Proof We compute

$$
\begin{aligned}
\|u\|_{\ell_{h}^{2}}^{2} & =h \sum_{j \in \mathbb{Z}} u(j h)^{2} \\
& =\sum_{j \in \mathbb{Z}} \int_{0}^{h} u(j h)^{2} d s \\
& =\sum_{j \in \mathbb{Z}} \int_{0}^{h}\left[u(j h+s)-\int_{0}^{s} u^{\prime}(j h+\sigma) d \sigma\right]^{2} d s .
\end{aligned}
$$

Using the standard bound $(a-b)^{2} \leq 2\left(a^{2}+b^{2}\right)$ we hence obtain

$$
\begin{aligned}
\|u\|_{\ell_{h}^{2}}^{2} \leq & 2 \sum_{j \in \mathbb{Z}} \int_{0}^{h} u(j h+s)^{2} d s \\
& +2 \sum_{j \in \mathbb{Z}} \int_{0}^{h}\left[\int_{0}^{s} u^{\prime}(j h+\sigma)^{2} d \sigma\right]^{2} d s \\
\leq & 2\|u\|_{L^{2}}^{2}+2 \sum_{j \in \mathbb{Z}} \int_{0}^{h} s \int_{0}^{s} u^{\prime}(j h+\sigma)^{2} d \sigma d s
\end{aligned}
$$




$$
\begin{aligned}
& =2\|u\|_{L^{2}}^{2}+2 \sum_{j \in \mathbb{Z}} \int_{0}^{h} u^{\prime}(j h+\sigma)^{2} \int_{\sigma}^{h} s d s d \sigma \\
& \leq 2\|u\|_{L^{2}}^{2}+h^{2} \sum_{j \in \mathbb{Z}} \int_{0}^{h} u^{\prime}(j h+\sigma)^{2} d \sigma \\
& =2\|u\|_{L^{2}}^{2}+h^{2}\left\|u^{\prime}\right\|_{L^{2}}^{2} .
\end{aligned}
$$

Corollary A.3 There exists $K>0$ so that for any $\vartheta \in \mathbb{R}$, any $v \in H^{1}$ and any $0<h<1$, we have the bounds

$$
\begin{aligned}
\left\|\mathrm{ev}_{\vartheta} v\right\|_{\ell_{h}^{\infty}} & \leq K\|v\|_{H^{1}}, \\
\left\|\mathrm{ev}_{\vartheta} v\right\|_{\ell_{h}^{\infty ; 1}} & \leq K\left[\|v\|_{H^{1}}+\left\|\partial_{h}^{+} v\right\|_{H^{1}}\right], \\
\left\|\operatorname{ev}_{\vartheta} v\right\|_{\ell_{h}^{\infty ; 2}} & \leq K\left[\|v\|_{H^{1}}+h^{-1 / 2}\left\|\partial_{h}^{+} v\right\|_{H^{1}}\right],
\end{aligned}
$$

together with

$$
\begin{aligned}
& \left\|\operatorname{ev}_{\vartheta} v\right\|_{\ell_{h}^{2 ; 1}} \leq K\|v\|_{H^{1}}, \\
& \left\|\operatorname{ev}_{\vartheta} v\right\|_{\ell_{h}^{2 ; 2}} \leq K\left[\|v\|_{H^{1}}+\left\|\partial_{h}^{+} v\right\|_{H^{1}}\right] .
\end{aligned}
$$

Proof For convenience, pick $\vartheta=0$. Using Lemma A.1 and the standard Sobolev bound $\|v\|_{\infty} \leq C_{1}\|v\|_{H^{1}}$ for some $C_{1}>0$, we find

$$
\begin{aligned}
\|v\|_{\ell_{h}^{\infty}} & \leq C_{1}\|v\|_{H^{1}}, \\
\left\|\partial_{h}^{+} v\right\|_{\ell_{h}^{\infty}} & \leq C_{1}\left\|\partial_{h}^{+} v\right\|_{H^{1}}, \\
\left\|\partial_{h}^{+} \partial_{h}^{+} v\right\|_{\ell_{h}^{\infty}} & \leq h^{-1 / 2}\left\|\partial_{h}^{+} v^{\prime}\right\|_{L^{2}} \\
& \leq h^{-1 / 2}\left\|\partial_{h}^{+} v\right\|_{H^{1}} .
\end{aligned}
$$

In addition, using (A.6) we find

$$
\begin{aligned}
\|v\|_{\ell_{h}^{2}} & \leq 3\|v\|_{H^{1}}, \\
\left\|\partial_{h}^{+} v\right\|_{\ell_{h}^{2}} & \leq\left\|v^{\prime}\right\|_{L^{2}} \\
& \leq\|v\|_{H^{1}}, \\
\left\|\partial_{h}^{+} \partial_{h}^{+} v\right\|_{\ell_{h}^{2}} & \leq\left\|\partial_{h}^{+} v^{\prime}\right\|_{L^{2}} \\
& \leq\left\|\partial_{h}^{+} v\right\|_{H^{1}} .
\end{aligned}
$$

We remark that the results above show that we automatically have $\operatorname{ev}_{\vartheta} u \in \ell_{h}^{2}$ whenever $u \in H^{1}$. We exploit this in the next result, which shows how to recover $L^{2}$ norms from the individual grid evaluations. We note that a direct consequence of (A.3) and (i) below is that we have

$$
\left\|\partial_{h}^{ \pm} u\right\|_{L^{q}} \leq\left\|u^{\prime}\right\|_{L^{q}}
$$

for any $u \in H^{1}$ and $q \in\{2, \infty\}$. 
Lemma A.4 Consider any $f \in C(\mathbb{R} ; \mathbb{R})$ and any $g \in H^{1}$. Then the following properties hold for all $h>0$.

(i) If the bound

$$
\|\operatorname{ev} \vartheta f\|_{\ell_{h}^{2}} \leq\|g\|_{\infty}
$$

holds for all $\vartheta \in[0, h]$, then $f \in L^{2}$ with

$$
\|f\|_{L^{2}} \leq\|g\|_{\infty} .
$$

(ii) If the bound

$$
\|\operatorname{ev} \vartheta f\|_{\ell_{h}^{2}} \leq\left\|\operatorname{ev}_{\vartheta} g\right\|_{\ell_{h}^{2}}
$$

holds for all $\vartheta \in[0, h]$, then $f \in L^{2}$ with

$$
\|f\|_{L^{2}} \leq\|g\|_{L^{2}} .
$$

(iii) If the bound

$$
\left\|\mathrm{ev}_{\vartheta} f\right\|_{\ell_{h}^{2}} \leq\left\|\mathrm{ev}_{\vartheta} g\right\|_{\ell_{h}^{2 ; 2}}
$$

holds for all $\vartheta \in(0, h)$, then $f \in L^{2}$ with

$$
\|f\|_{L^{2}} \leq\|g\|_{H^{1}}+\left\|\partial_{h}^{+} \partial_{h}^{+} g\right\|_{L^{2}} .
$$

(iv) If the bound

$$
\left\|\operatorname{ev}_{\vartheta} f\right\|_{\ell_{h}^{2}} \leq\left\|\operatorname{ev}_{\vartheta} g\right\|_{\ell_{h}^{2 ; 3}}
$$

holds for all $\vartheta \in[0, h]$, then $f \in L^{2}$ with

$$
\|f\|_{L^{2}} \leq\|g\|_{H^{1}}+\left\|\partial_{h}^{+} g\right\|_{H^{1}}+\left\|\partial_{h}^{+} \partial_{h}^{+} \partial_{h}^{+} g\right\| .
$$

Proof We first note that

$$
\begin{aligned}
\|f\|_{L^{2}}^{2} & =\int_{\mathbb{R}} f(x)^{2} d x \\
& =\sum_{k \in \mathbb{Z}} \int_{0}^{h} f(k h+\vartheta)^{2} d \vartheta \\
& =h^{-1} \int_{0}^{h}\left\|\mathrm{ev}_{\vartheta} f\right\|_{\ell_{h}^{2}}^{2} d \vartheta
\end{aligned}
$$

Item (i) and (ii) follow immediately from this.

For (iii), we note

$$
\begin{aligned}
\|f\|_{L^{2}}^{2} & \leq h^{-1} \int_{0}^{h}\left\|\mathrm{ev}_{\vartheta} g\right\|_{\ell_{h}^{2 ; 2}}^{2} d \vartheta \\
& =h^{-1} \int_{0}^{h}\left[\left\|\mathrm{ev}_{\vartheta} g\right\|_{\ell_{h}^{2}}^{2}+\left\|\mathrm{ev}_{\vartheta} \partial_{h}^{+} g\right\|_{\ell_{h}^{2}}^{2}+\left\|\mathrm{ev}_{\vartheta} \partial_{h}^{+} \partial_{h}^{+} g\right\|_{\ell_{h}^{2}}^{2}\right] d \vartheta \\
& =\|g\|_{L^{2}}^{2}+\left\|\partial_{h}^{+} g\right\|_{L^{2}}^{2}+\left\|\partial_{h}^{+} \partial_{h}^{+} g\right\|_{L^{2}}^{2} .
\end{aligned}
$$

Exploiting (A.13), we obtain

$$
\|f\|_{L^{2}}^{2} \leq\|g\|_{H^{1}}^{2}+\left\|\partial_{h}^{+} \partial_{h}^{+} g\right\|_{L^{2}}^{2}
$$


as desired.

To see (iv), we apply (A.13) to $\partial_{h}^{+} g$ to obtain

$$
\left\|\partial_{h}^{+} \partial_{h}^{+} g\right\|_{L^{2}} \leq\left\|\partial_{h}^{+} g^{\prime}\right\|_{L^{2}} .
$$

This yields the desired bound

$$
\begin{aligned}
\|f\|_{L^{2}}^{2} & \leq\|g\|_{L^{2}}^{2}+\left\|\partial_{h}^{+} g\right\|_{L^{2}}^{2}+\left\|\partial_{h}^{+} \partial_{h}^{+} g\right\|_{L^{2}}^{2}+\left\|\partial_{h}^{+} \partial_{h}^{+} \partial_{h}^{+} g\right\|_{L^{2}}^{2} \\
& \leq\|g\|_{L^{2}}^{2}+\left\|\partial_{h}^{+} g\right\|_{L^{2}}^{2}+\left\|\partial_{h}^{+} g^{\prime}\right\|_{L^{2}}^{2}+\left\|\partial_{h}^{+} \partial_{h}^{+} \partial_{h}^{+} g\right\|_{L^{2}}^{2} \\
& \leq\|g\|_{H^{1}}^{2}+\left\|\partial_{h}^{+} g\right\|_{H^{1}}^{2}+\left\|\partial_{h}^{+} \partial_{h}^{+} \partial_{h}^{+} g\right\|_{L^{2}}^{2} .
\end{aligned}
$$

\section{B The State Space $\boldsymbol{\Omega}_{h ; \kappa}$}

In this section we provide the proofs for Propositions 3.1-3.3. The main idea is to use the sampling estimates from $\S$ A to exploit the following key scaling result.

Lemma B. 1 For any $0<\kappa<1$ and $h>0$, we have the bounds

$$
\left\|U_{\text {ref } ; \kappa}\right\|_{\ell_{h}^{\infty}} \leq 1, \quad\left\|\partial^{+} U_{\text {ref; } \kappa}\right\|_{\ell_{h}^{\infty}} \leq \kappa, \quad\left\|\partial^{+} \partial^{+} U_{\text {ref } ; \kappa}\right\|_{\ell_{h}^{\infty}} \leq \kappa^{2},
$$

together with

$$
\left\|\partial^{+} U_{\text {ref } ; \kappa}\right\|_{\ell_{h}^{2}} \leq 2 \kappa^{1 / 2}, \quad\left\|\partial^{+} \partial^{+} U_{\text {ref } ; \kappa}\right\|_{\ell_{h}^{2}} \leq 2 \kappa^{3 / 2}
$$

and finally

$$
\left\|U_{\text {ref } ; \kappa}-H\right\|_{\ell_{h}^{2}} \leq 2 \sqrt{2} \kappa^{-1 / 2} .
$$

Proof The uniform bound on $U_{\text {ref; } \kappa}$ follows directly from the definition (3.36)-(3.37). Upon computing

$$
U_{\text {ref; } \kappa}^{\prime}(\xi)=\kappa U_{\text {ref; } *}^{\prime}(\kappa \xi), \quad U_{\text {ref; } \kappa}^{\prime \prime}(\xi)=\kappa^{2} U_{\text {ref;* }}^{\prime \prime}(\kappa \xi),
$$

the properties (3.37) immediately yield

$$
\left\|U_{\text {ref; } \kappa}^{\prime}\right\|_{L^{\infty}} \leq \kappa, \quad\left\|U_{\text {ref; } \kappa}^{\prime \prime}\right\|_{L^{\infty}} \leq \kappa^{2} .
$$

The bounds (B.1) now follow from (A.3) and (A.13).

We first observe that

$$
\left\|U_{\text {ref } ; *}^{\prime}\right\|_{L^{2}}^{2} \leq 4, \quad\left\|U_{\text {ref } ; *}^{\prime \prime}\right\|_{L^{2}}^{2} \leq 4 .
$$

This allows us to compute

$$
\begin{aligned}
\left\|U_{\text {ref } ; \kappa}^{\prime}\right\|_{L^{2}}^{2} & =\int \kappa^{2}\left[U_{\text {ref; } *}^{\prime}(\kappa \tau)\right]^{2} d \tau \\
& =\kappa \int\left[U_{\text {ref } ; *}^{\prime}\left(\tau^{\prime}\right)\right]^{2} d \tau^{\prime} \\
& =\kappa\left\|U_{\text {ref } ; *}^{\prime}\right\|_{L^{2}}^{2} \\
& \leq 4 \kappa .
\end{aligned}
$$


In a similar fashion, we obtain

$$
\left\|U_{\text {ref } ; \kappa}^{\prime \prime}\right\|_{L^{2}}^{2}=\kappa^{3}\left\|U_{\text {ref;* }}^{\prime}\right\|_{L^{2}}^{2} \leq 4 \kappa^{3} .
$$

We may now apply (A.3) and (A.13) once more to obtain (B.2).

Since $U_{\text {ref; } \kappa}$ is an increasing function, we see that

$$
\begin{aligned}
h \sum_{j<0} U_{\mathrm{ref} ; \kappa}(j h)^{2} & \leq \int_{-\infty}^{0} U_{\mathrm{ref} ; \kappa}(\tau+h)^{2} d \tau \\
& =\int_{-\infty}^{0} U_{\mathrm{ref} ; *}(\kappa(\tau+h))^{2} d \tau \\
& =\kappa^{-1} \int_{-\infty}^{\kappa h} U_{\mathrm{ref} ; *}\left(\tau^{\prime}\right)^{2} d \tau^{\prime} \\
& \leq \kappa^{-1} \int_{-2}^{2} U_{\mathrm{ref} ; *}\left(\tau^{\prime}\right)^{2} d \tau^{\prime} \\
& \leq 4 \kappa^{-1} .
\end{aligned}
$$

In a similar fashion, we find

$$
h \sum_{j \geq 0}\left(U_{\mathrm{ref} ; \kappa}(j h)-1\right)^{2} \leq 4 \kappa^{-1}
$$

and hence

$$
\left\|U_{\text {ref; } \kappa}-H\right\|_{\ell_{h}^{2}} \leq 2 \sqrt{2} \kappa^{-1 / 2}
$$

as desired.

Proof of Proposition 3.1 Write $U=U_{\text {ref; } \kappa}+V$ with $V \in \mathcal{V}_{h ; \kappa}$. Note that Lemma B.1 implies that

$$
\left\|U_{\text {ref; } \kappa}\right\|_{\ell_{h}^{\infty}}+\left\|\partial^{+} U_{\text {ref } ; \kappa}\right\|_{\ell_{h}^{2}}+\left\|\partial^{+} \partial^{+} U_{\text {ref } ; \kappa}\right\|_{\ell_{h}^{2}}+\left\|\partial^{+} \partial^{+} U_{\text {ref } ; \kappa}\right\|_{\ell_{h}^{\infty}} \leq 6 .
$$

In particular, we see that

$$
\|U\|_{\ell_{h}^{\infty}}+\left\|\partial^{+} U\right\|_{\ell_{h}^{2}}+\left\|\partial^{+} \partial^{+} U\right\|_{\ell_{h}^{2}}+\left\|\partial^{+} \partial^{+} U\right\|_{\ell_{h}^{\infty}}<6+\frac{1}{2} \kappa^{-1} \leq \kappa^{-1}
$$

since $0<\kappa \leq \frac{1}{12}$. In addition, we see that

$$
\left\|\partial^{+} U\right\|_{\ell_{h}^{\infty}} \leq\left\|\partial^{+} U_{\operatorname{ref} ; \kappa}\right\|_{\ell_{h}^{\infty}}+\left\|\partial^{+} V\right\|_{\ell_{h}^{\infty}}<\kappa+1-2 \kappa=1-\kappa,
$$

as desired.

Finally, we note that

$$
g(U)=g(H)+g\left(U_{\text {ref } ; \kappa}\right)-g(H)+g\left(U_{\text {ref } ; \kappa}+V\right)-g\left(U_{\text {ref } ; \kappa}\right) .
$$

Writing

$$
M=\sup _{|u| \leq \kappa^{-1}}\left|g^{\prime}(u)\right|
$$

we see that

$$
\left\|g\left(U_{\mathrm{ref} ; \kappa}\right)-g(H)\right\|_{\ell_{h}^{2}} \leq M
$$




$$
\begin{aligned}
\left\|U_{\text {ref } ; \kappa}-H\right\|_{\ell_{h}^{2}} & \leq 2 \sqrt{2} M \kappa^{-1 / 2}, \\
\left\|g\left(U_{\text {ref } ; \kappa}+V\right)-g\left(U_{\text {ref } ; \kappa}\right)\right\|_{\ell_{h}^{2}} & \leq M\|V\|_{\ell_{h}^{2}} \\
& \leq \frac{1}{2} M \kappa^{-1} .
\end{aligned}
$$

The desired bound now follows from $g(H)=0$.

Proof of Proposition 3.2 Notice first that $\partial^{+} H \in \ell_{h}^{2}$, which with $U-H \in \ell_{h}^{2}$ implies that $\partial^{+} U \in \ell_{h}^{2}$. Pick $\kappa>0$ to be so small that

$$
\left\|\partial^{+} U\right\|_{\ell_{h}^{\infty}}<1-4 \kappa
$$

and also

$$
\|U\|_{\ell_{h}^{\infty}}+\left\|\partial^{+} U\right\|_{\ell_{h}^{2}}+\left\|\partial^{+} \partial^{+} U\right\|_{\ell_{h}^{2}}+\left\|\partial^{+} \partial^{+} U\right\|_{\ell_{h}^{\infty}}+6<\frac{1}{8} \kappa^{-1} .
$$

In addition, pick $\epsilon_{0}>0$ to be so small that

$$
\left\|\partial^{+} \tilde{U}\right\|_{\ell_{h}^{\infty}}<1-3 \kappa
$$

and also

$$
\|\widetilde{U}\|_{\ell_{h}^{\infty}}+\left\|\partial^{+} \widetilde{U}\right\|_{\ell_{h}^{2}}+\left\|\partial^{+} \partial^{+} \widetilde{U}\right\|_{\ell_{h}^{2}}+\left\|\partial^{+} \partial^{+} \widetilde{U}\right\|_{\ell_{h}^{\infty}}+6<\frac{1}{4} \kappa^{-1}
$$

whenever $\|\widetilde{U}-U\|_{\ell_{h}^{2}}<\epsilon_{0}$, which is possible because of the continuous embedding $\ell_{h}^{2} \subset$ $\ell_{h}^{\infty}$.

For any such $\widetilde{U}$, we write

$$
V_{\kappa}=\tilde{U}-U_{\text {ref } ; \kappa}
$$

We immediately see

$$
\left\|\partial^{+} V_{\kappa}\right\|_{\ell_{h}^{\infty}} \leq\left\|\partial^{+} \tilde{U}\right\|_{\ell_{h}^{\infty}}+\left\|U_{\text {ref; } \kappa}\right\|_{\ell_{h}^{\infty}}<1-3 \kappa+\kappa=1-2 \kappa .
$$

In addition, we have

$$
\left\|V_{\kappa}\right\|_{\ell_{h}^{\infty}}+\left\|\partial^{+} V_{\kappa}\right\|_{\ell_{h}^{2}}+\left\|\partial^{+} \partial^{+} V_{\kappa}\right\|_{\ell_{h}^{2}}+\left\|\partial^{+} \partial^{+} V_{\kappa}\right\|_{\ell_{h}^{\infty}}<\frac{1}{4} \kappa^{-1}-6+6=\frac{1}{4} \kappa^{-1} .
$$

Finally, we note that

$$
\begin{aligned}
\left\|V_{\kappa}\right\|_{\ell_{h}^{2}} & =\left\|\widetilde{U}-U_{\text {ref; } \kappa}\right\|_{\ell_{h}^{2}} \\
& \leq\|\widetilde{U}-U\|_{\ell_{h}^{2}}+\|U-H\|_{\ell_{h}^{2}}+\left\|U_{\text {ref; } \kappa}-H\right\|_{\ell_{h}^{2}} \\
& \leq \epsilon_{0}+\|U-H\|_{\ell_{h}^{2}}+2 \sqrt{2} \kappa^{-1 / 2} .
\end{aligned}
$$

By decreasing $\kappa>0$ even further, which does not destroy the estimates above, we can hence obtain

$$
\left\|V_{\kappa}\right\|_{\ell_{h}^{2}}<\frac{1}{4} \kappa^{-1}
$$

This shows that $V_{\kappa} \in \mathcal{V}_{h ; \kappa}$, as desired. 
Proof of Proposition 3.3 Pick $\kappa>0$ to be so small that

$$
\left\|u^{\prime}\right\|_{L^{\infty}}<1-4 \kappa
$$

and also

$$
\|u\|_{H^{1}}+\left\|u^{\prime}\right\|_{L^{2}}+\left\|u^{\prime \prime}\right\|_{L^{2}}+\left\|u^{\prime \prime}\right\|_{H^{1}}+6<\frac{1}{8} \kappa^{-1} .
$$

Using Lemma A.1 and the inequality (A.13), we obtain

$$
\left\|\operatorname{ev}_{\vartheta} \partial_{h}^{+} u\right\|_{\ell_{h}^{\infty}} \leq\left\|u^{\prime}\right\|_{L^{\infty}}<1-4 \kappa
$$

together with

$$
\left\|\operatorname{ev}_{\vartheta} u\right\|_{\ell_{h}^{\infty}}+\left\|\operatorname{ev}_{\vartheta} \partial_{h}^{+} u\right\|_{\ell_{h}^{2}}+\left\|\operatorname{ev}_{\vartheta} \partial_{h}^{+} \partial_{h}^{+} u\right\|_{\ell_{h}^{2}}+\left\|\operatorname{ev}_{\vartheta} \partial_{h}^{+} \partial_{h}^{+} u\right\|_{\ell_{h}^{\infty}}+6<\frac{1}{8} \kappa^{-1}(\mathrm{~B}
$$

for any $\vartheta \in \mathbb{R}$.

Corollary A.3 implies that we can pick a small constant $\epsilon_{0}>0$ in such a way that

$$
\left\|\mathrm{ev}_{\vartheta} v\right\|_{\ell_{h}^{\infty ; 2}}+\left\|\mathrm{ev}_{\vartheta} v\right\|_{\ell_{h}^{2 ; 2}}<\min \left\{\kappa, \frac{1}{8} \kappa^{-1}\right\}
$$

holds for every $\vartheta \in \mathbb{R}$ and any $v \in H^{1}$ that satisfies (3.46). Upon writing $w=u+v$ for any such $v$, we see that

$$
\left\|\mathrm{ev}_{\vartheta} w\right\|_{\ell_{h}^{\infty}}<1-4 \kappa+\kappa<1-3 \kappa
$$

together with

$$
\left\|\operatorname{ev}_{\vartheta} w\right\|_{\ell_{h}^{\infty}}+\left\|\operatorname{ev}_{\vartheta} \partial_{h}^{+} w\right\|_{\ell_{h}^{2}}+\left\|\operatorname{ev}_{\vartheta} \partial_{h}^{+} \partial_{h}^{+} w\right\|_{\ell_{h}^{2}}+\left\|\operatorname{ev}_{\vartheta} \partial_{h}^{+} \partial_{h}^{+} w\right\|_{\ell_{h}^{\infty}}+6<\frac{1}{4} \kappa^{-1}(
$$

for any $\vartheta \in \mathbb{R}$.

For any such $w$, we write

$$
V_{\kappa ; \vartheta}=\mathrm{ev}_{\vartheta} w-U_{\mathrm{ref} ; \kappa} .
$$

We immediately see

$$
\left\|\partial^{+} V_{\kappa ; \vartheta}\right\|_{\ell_{h}^{\infty}} \leq\left\|\operatorname{ev}_{\vartheta} \partial_{h}^{+} w\right\|_{\ell_{h}^{\infty}}+\left\|\partial_{h}^{+} U_{\mathrm{ref} ; \kappa}\right\|_{\ell_{h}^{\infty}}<1-3 \kappa+\kappa=1-2 \kappa .
$$

In addition, we have

$$
\left\|V_{\kappa ; \vartheta}\right\|_{\ell_{h}^{\infty}}+\left\|\partial^{+} V_{\kappa ; \vartheta}\right\|_{\ell_{h}^{2}}+\left\|\partial^{+} \partial^{+} V_{\kappa ; \vartheta}\right\|_{\ell_{h}^{2}}+\left\|\partial^{+} \partial^{+} V_{\kappa ; \vartheta}\right\|_{\ell_{h}^{\infty}}<\frac{1}{4} \kappa^{-1}-6+6=\frac{1}{4} \kappa^{-1} \text {. }
$$

Finally, we note that

$$
\begin{aligned}
& \left\|V_{\kappa ; \vartheta}\right\|_{\ell_{h}^{2}}=\left\|\mathrm{ev}_{\vartheta} w-U_{\text {ref } ; \kappa}\right\|_{\ell_{h}^{2}} \\
& \leq\left\|\mathrm{ev}_{\vartheta} w-\operatorname{ev}_{\vartheta} u\right\|_{\ell_{h}^{2}}+\left\|\mathrm{ev}_{\vartheta} u-\operatorname{ev}_{\vartheta} U_{\mathrm{ref} ; *}\right\|_{\ell_{h}^{2}} \\
& +\left\|\mathrm{ev}_{\vartheta} U_{\mathrm{ref} ; *}-U_{\mathrm{ref} ; *}\right\|_{\ell_{h}^{2}}+\left\|U_{\mathrm{ref} ; *}-H\right\|_{\ell_{h}^{2}}+\left\|U_{\mathrm{ref} ; \kappa}-H\right\|_{\ell_{h}^{2}} \\
& \leq \epsilon_{0}+3\left\|u-U_{\text {ref } ; *}\right\|_{H^{1}}+3\left\|U_{\text {ref } ; *}(\cdot+\vartheta)-U_{\text {ref } ; *}(\cdot)\right\|_{H^{1}}+2 \sqrt{2}+2 \sqrt{2} \kappa^{-1 / 2} .
\end{aligned}
$$


By decreasing $\kappa>0$ even further, which does not destroy the estimates above, we can hence obtain

$$
\left\|V_{\kappa ; \vartheta}\right\|_{\ell_{h}^{2}}<\frac{1}{4} \kappa^{-1}
$$

for all $\vartheta \in[0, h]$. This shows that $V_{\kappa ; \vartheta} \in \mathcal{V}_{h ; \kappa}$ for all $\vartheta \in[0, h]$, as desired.

\section{Preliminary Identities}

Our goal here is to obtain a number of useful preliminary identities for the gridspace functions $r_{U}^{ \pm}, r_{U}^{0}$ and $\gamma_{U}$ defined in (3.16) and (7.6). In addition, we verify the representations (3.23) and (3.29) for the discrete derivatives $\mathcal{D}_{-;}^{\diamond_{+}}, \mathcal{D}^{\diamond_{0} ;+}$ and $\mathcal{D}^{\diamond_{0} ;+}$. The computations are relatively direct, based on the discrete calculus outlined in $\S 3.1$.

Lemma C.1 Consider any $U \in \ell^{\infty}(h \mathbb{Z} ; \mathbb{R})$ for which $\left\|\partial^{+} U\right\|_{\infty}<1$. Then we have the identities

$$
\begin{aligned}
& \partial^{+} r_{U}^{-}=-\left[r_{U}^{0}\right]^{-1} \partial^{0} U \partial^{(2)} U, \\
& \partial^{+} r_{U}^{0}=-S^{+}\left[\left[r_{U}^{0}\right]^{-1} \partial^{0} U \partial^{(2)} U\right] .
\end{aligned}
$$

Proof We compute

$$
\begin{aligned}
r_{U}^{+}-r_{U}^{-} & =\sqrt{1-\left(\partial^{+} U\right)^{2}}-\sqrt{1-\left(\partial^{-} U\right)^{2}} \\
& =\frac{\left(\partial^{-} U\right)^{2}-\left(\partial^{+} U\right)^{2}}{r^{+}+r^{-}} \\
& =\frac{-\left(\partial^{+} U-\partial^{-} U\right)\left(\partial^{+} U+\partial^{-} U\right)}{2 r_{U}^{0}} \\
& =\frac{-h \partial^{(2)} U\left(2 \partial^{0} U\right)}{2 r_{U}^{0}},
\end{aligned}
$$

from which the first identity follows. In addition, we see that

$$
\begin{aligned}
h \partial^{+}\left[r_{U}^{0}\right] & =T^{+} r_{U}^{0}-r_{U}^{0} \\
& =\frac{1}{2}\left[T^{+} r_{U}^{+}+T^{+} r_{U}^{-}-r_{U}^{+}-r_{U}^{-}\right] \\
& =\frac{1}{2}\left[T^{+} r_{U}^{+}+r_{U}^{+}-T^{+} r_{U}^{-}-r_{U}^{-}\right] \\
& =\frac{1}{2} T^{+}\left[r_{U}^{+}-r_{U}^{-}\right]+\frac{1}{2}\left[r_{U}^{+}-r_{U}^{-}\right] \\
& =S^{+}\left[r_{U}^{+}-r_{U}^{-}\right] .
\end{aligned}
$$

Using (3.19) we conclude $\partial^{+}\left[r_{U}^{0}\right]=S^{+}\left[\partial^{+} r_{U}^{-}\right]$, which yields the second identity.

Recalling (7.6), a short computation shows that

$$
\begin{aligned}
\gamma_{U^{(b)}}-\gamma_{U^{(a)}} & =\sqrt{1-\left(\partial^{0} U^{(b)}\right)^{2}}-\sqrt{1-\left(\partial^{0} U^{(a)}\right)^{2}} \\
& =-\frac{\left(\partial^{0} U^{(b)}\right)^{2}-\left(\partial^{0} U^{(a)}\right)^{2}}{\sqrt{1-\left(\partial^{0} U^{(a)}\right)^{2}}+\sqrt{1-\left(\partial^{0} U^{(b)}\right)^{2}}}
\end{aligned}
$$




$$
=-\left[\gamma_{U^{(a)}}+\gamma_{U^{(b)}}\right]^{-1}\left(\partial^{0} U^{(a)}+\partial^{0} U^{(b)}\right)\left(\partial^{0} U^{(b)}-\partial^{0} U^{(a)}\right),
$$

which allows us to compute several useful discrete derivatives.

Lemma C.2 Consider any $U \in \ell^{\infty}(h \mathbb{Z} ; \mathbb{R})$ for which $\left\|\partial^{+} U\right\|_{\infty}<1$. Then we have the identities

$$
\begin{aligned}
\partial^{+}\left[\gamma_{U}^{-4}\right] & =\frac{4 S^{+}\left[\partial^{0} U\right] S^{+}\left[\partial^{(2)} U\right]}{P^{+}\left[\gamma_{U}^{2}\right]} \frac{S^{+}\left[\gamma_{U}^{2}\right]}{P^{+}\left[\gamma_{U}^{2}\right]}, \\
\partial^{+}\left[\gamma_{U}^{-2}\right] & =\frac{2 S^{+}\left[\partial^{0} U\right] S^{+}\left[\partial^{(2)} U\right]}{P^{+}\left[\gamma_{U}^{2}\right]}, \\
\partial^{+}\left[\gamma_{U}^{-1}\right] & =\frac{S^{+}\left[\partial^{0} U\right] S^{+}\left[\partial^{(2)} U\right]}{S^{+}\left[\gamma_{U}\right] P^{+}\left[\gamma_{U}\right]}, \\
\partial^{+}\left[\gamma_{U}\right] & =-\frac{S^{+}\left[\partial^{0} U\right] S^{+}\left[\partial^{(2)} U\right]}{S^{+} \gamma_{U}}, \\
\partial^{+}\left[\gamma_{U}^{2}\right] & =-2 S^{+}\left[\partial^{0} U\right] S^{+}\left[\partial^{(2)} U\right] .
\end{aligned}
$$

Proof Writing $U^{(2)}=T^{+} U$ and $U^{(1)}=U$, we use (C.4) to compute

$$
h \partial^{+} \gamma_{U}=-\left[S^{+} \gamma_{U}\right]^{-1} S^{+}\left[\partial^{0} U\right] h \partial^{+} \partial^{0} U
$$

which yields the desired identity for $\partial^{+} \gamma_{U}$ upon remembering (3.4). We can now use the general identities

$$
\begin{aligned}
\partial^{+}\left[a^{-1}\right] & =-\left[P^{+} a\right]^{-1} \partial^{+} a, \\
\partial^{+}\left[a^{2}\right] & =\left[\partial^{+} a\right] T^{+} a+a \partial^{+} a=2\left[\partial^{+} a\right] S^{+} a,
\end{aligned}
$$

together with

$$
S^{+}\left[a^{-1}\right]=\frac{S^{+} a}{P^{+} a}
$$

to obtain the remaining expressions.

Lemma C.3 Consider any $U \in \ell^{\infty}(h \mathbb{Z} ; \mathbb{R})$ for which $\left\|\partial^{+} U\right\|_{\infty}<1$ and recall the expressions (3.22). Then the second derivatives defined in (3.21) satisfy the identities

$$
\begin{aligned}
& \mathcal{D}^{\diamond_{-} ;+}(U)=\mathcal{I}_{0}^{\diamond_{-} ;+}(U) \partial^{(2)} U, \\
& \mathcal{D}^{\diamond_{0} ;+}(U)=\mathcal{I}_{0}^{\diamond_{0} ;+}(U) \partial^{(2)} U+\mathcal{I}_{S}^{\diamond_{0} ;+}(U) T^{+}\left[\partial^{(2)} U\right] .
\end{aligned}
$$

Proof Using (3.4), (3.7) and (3.20) we compute

$$
\begin{aligned}
\mathcal{D}^{{ }_{-} ;+}(U) & =\left[P^{+} r_{U}^{-}\right]^{-1}\left[r_{U}^{-} \partial^{+} \partial^{-} U-\partial^{-} U \partial^{+} r_{U}^{-}\right] \\
& =\left[r_{U}^{-} r_{U}^{+}\right]^{-1}\left[r_{U}^{-} \partial^{(2)} U+\partial^{-} U \mathcal{D}^{\diamond}(U) \partial^{(2)} U\right] \\
& =\left[r_{U}^{+}\right]^{-1}\left[\partial^{(2)} U+\mathcal{D}^{\diamond-}(U) \mathcal{D}^{\diamond}(U) \partial^{(2)} U\right],
\end{aligned}
$$

together with

$$
\begin{aligned}
\mathcal{D}^{\diamond_{0} ;+}(U) & =\left[P^{+} r_{U}^{0}\right]^{-1}\left[r_{U}^{0} \partial^{+} \partial^{0} U-\partial^{0} U \partial^{+} r_{U}^{0}\right] \\
& =\left[r_{U}^{0} T^{+} r_{U}^{0}\right]^{-1}\left[r_{U}^{0} S^{+} \partial^{(2)} U+\partial^{0} U S^{+}\left[\mathcal{D}^{\diamond_{0}}(U) \partial^{(2)} U\right]\right]
\end{aligned}
$$




$$
=\left[T^{+} r_{U}^{0}\right]^{-1}\left[S^{+} \partial^{(2)} U+\mathcal{D}^{\diamond_{0}}(U) S^{+}\left[\mathcal{D}^{\diamond_{0}}(U) \partial^{(2)} U\right]\right],
$$

from which the desired identities follow.

Lemma C.4 Consider any $U \in \ell^{\infty}(h \mathbb{Z} ; \mathbb{R})$ for which $\left\|\partial^{+} U\right\|_{\infty}<1$ and recall the expressions (3.28)-(3.29). Then the third derivative (3.27) satisfies the identity

$$
\mathcal{D}^{\diamond \diamond_{0} ;+}(U)=\mathcal{D}_{a}^{\diamond_{0} ;+}(U)+\mathcal{D}_{b}^{\diamond_{0} ;+}(U) .
$$

Proof Using (3.26) we may compute

$$
\begin{aligned}
\partial^{+} \mathcal{D}^{\diamond_{0}}(U) & =\partial^{+}\left[\frac{1}{r_{U}^{0} r_{U}^{+}}\left(1+\mathcal{D}^{\diamond}(U) \mathcal{D}^{\diamond_{0}}(U)\right) \partial^{(2)} U\right] \\
& =\mathcal{I}_{A}+\mathcal{I}_{B}+\mathcal{I}_{C},
\end{aligned}
$$

in which

$$
\begin{aligned}
& \mathcal{I}_{A}=\partial^{+}\left[\frac{1}{r_{U}^{0} r_{U}^{+}}\right] T^{+}\left[\left(1+\mathcal{D}^{\diamond}(U) \mathcal{D}^{\diamond_{0}}(U)\right) \partial^{(2)} U\right] \\
& \mathcal{I}_{B}=\frac{1}{r_{U}^{0} r_{U}^{+}}\left(\mathcal{D}^{\diamond-;+}(U) T^{+} \mathcal{D}^{\diamond_{0}}(U)+\mathcal{D}^{\diamond}(U) \mathcal{D}^{\diamond_{0} ;+}(U)\right) T^{+}\left[\partial^{(2)} U\right], \\
& \mathcal{I}_{C}=\frac{1}{r_{U}^{0} r_{U}^{+}}\left(1+\mathcal{D}^{\diamond}(U) \mathcal{D}^{\diamond_{0}}(U)\right) \partial^{+} \partial^{(2)} U .
\end{aligned}
$$

We immediately see that

$$
\mathcal{I}_{C}=\mathcal{I}_{+}^{\diamond \diamond_{0} ;+}(U) \partial^{+} \partial^{(2)} U .
$$

In addition, we may use (3.7) and (3.20) to compute

$$
\begin{aligned}
\partial^{+}\left[\frac{1}{r_{U}^{0} r_{U}^{+}}\right] & =-\left[P^{+} r_{U}^{0} P^{+} r_{U}^{+}\right]^{-1}\left[\partial^{+} r_{U}^{0} T^{+} r^{+}+r_{U}^{0} \partial^{+} r^{+}\right] \\
& =\left[P^{+} r_{U}^{0} P^{+} r_{U}^{+}\right]^{-1}\left[S^{+}\left[\mathcal{D}^{\diamond}(U) \partial^{(2)} U\right] T^{+} r_{U}^{+}+\frac{1}{2} r_{U}^{0} T^{+}\left[\mathcal{D}^{\diamond}(U) \partial^{(2)} U\right]\right] \\
& =\left[\frac{1}{2 r_{U}^{+} P^{+} r_{U}^{0}} \mathcal{D}^{\diamond_{0}}(U)\right] \partial^{(2)} U+\left[\frac{2 r_{U}^{0}+T^{+} r_{U}^{+}}{2 P^{+} r_{U}^{0} P^{+} r_{U}^{+}}\right] T^{+}\left[\mathcal{D}^{\diamond}(U) \partial^{(2)} U\right] .(\mathrm{C} .16)
\end{aligned}
$$

Finally, Lemma C.3 allows us to expand

$$
\begin{aligned}
& \mathcal{I}_{B}=\left[r_{U}^{0} r_{U}^{+} r_{U}^{+}\right]^{-1}\left[1+\mathcal{D}^{\diamond}-(U) \mathcal{D}^{\diamond}(U)\right]\left[\partial^{(2)} U\right] T^{+}\left[\mathcal{D}^{\diamond}(U) \partial^{(2)} U\right] \\
& +\left[2 r_{U}^{+} P^{+} r_{U}^{0}\right]^{-1}\left[1+\mathcal{D}^{\diamond_{0}}(U) \mathcal{D}^{\diamond_{0}}(U)\right] \mathcal{D}^{\diamond}-(U)\left[\partial^{(2)} U\right] T^{+}\left[\partial^{(2)} U\right] \\
& +\left[2 r_{U}^{+} P^{+} r_{U}^{0}\right]^{-1}\left[1+\mathcal{D}^{\diamond 0}(U) T^{+} \mathcal{D}^{\diamond 0}(U)\right] \mathcal{D}^{\diamond-}(U) T^{+}\left[\partial^{(2)} U\right] T^{+}\left[\partial^{(2)} U\right] \text {. }
\end{aligned}
$$

The splitting (C.12) can now be read off directly.

\section{Preliminary Estimates}

In this section we exploit the bounds in Proposition 3.1 to obtain a number of technical estimates on the gridpoint spacing functions and discrete derivatives that were introduced in $\S 3$. These bounds will help to streamline the computations in the rest of the appendices. 


\section{D.1 Gridpoint Spacing Estimates}

Our first result here is crucial as it shows that the inverse functions $\left[r_{U}^{ \pm}\right]^{-1}$ and $\gamma_{U}^{-1}$ can be uniformly bounded on $\Omega_{h ; \kappa}$ for all $h>0$ simultaneously. We use it to simplify the expressions for $\gamma_{U}^{-k}$ defined in Lemma C.2 at the cost of an $O(h)$ error term.

Lemma D.1 Fix $h>0$ and $0<\kappa<\frac{1}{12}$. Then for any $U \in \Omega_{h ; \kappa}$, we have the pointwise estimates

$$
\sqrt{\kappa}<r_{U}^{ \pm} \leq 1, \quad \sqrt{\kappa}<\gamma_{U} \leq 1
$$

Proof We compute

$$
1 \geq \sqrt{1-\left(\partial^{ \pm} U\right)^{2}}>\sqrt{1-(1-\kappa)^{2}}=\sqrt{1-1+2 \kappa-\kappa^{2}} \geq \sqrt{\kappa} .
$$

Corollary D.2 Fix $0<\kappa<\frac{1}{12}$. Then there exists $K>0$ so that for any $h>0$ and any pair $\left(U^{(a)}, U^{(b)}\right) \in \Omega_{h ; \kappa}^{2}$, we have the estimates

$$
\begin{gathered}
\left\|\gamma_{U^{(a)}}-\gamma_{U^{(b)}}\right\|_{\ell_{h}^{\infty}} \leq K\left\|\partial^{+} U^{(b)}-\partial^{+} U^{(a)}\right\|_{\ell_{h}^{\infty}}, \\
\left\|\gamma_{U^{(a)}}-\gamma_{U^{(b)}}\right\|_{\ell_{h}^{2}} \leq K\left\|\partial^{+} U^{(b)}-\partial^{+} U^{(a)}\right\|_{\ell_{h}^{2}} .
\end{gathered}
$$

Proof These bounds are a direct consequence of the lower bounds in (D.1) and the representation (C.4).

Corollary D.3 Fix $0<\kappa<\frac{1}{12}$. Then there exists $K>0$ so that for any $h>0$ and any $U \in \Omega_{h ; \kappa}$ we have the bounds

$$
\begin{aligned}
& \left|\partial^{+} r_{U}^{-}\right| \leq K\left|\partial^{(2)} U\right| \\
& \left|\partial^{+} r_{U}^{0}\right| \leq K\left[\left|\partial^{(2)} U\right|+T^{+}\left|\partial^{(2)} U\right|\right] .
\end{aligned}
$$

Proof These estimates follow directly from Lemma C.1.

Lemma D.4 Fix $0<\kappa<\frac{1}{12}$. Then there exists $K>0$ so that for any $h>0$ and any $U \in \Omega_{h ; \kappa}$, we have the pointwise estimates

$$
\begin{aligned}
\left|\partial^{+}\left[\gamma_{U}^{2}\right]+2 \partial^{0} U S^{+}\left[\partial^{(2)} U\right]\right| & \leq K h\left[\left|\partial^{(2)} U\right|^{2}+T^{+}\left|\partial^{(2)} U\right|^{2}\right], \\
\left|\partial^{+}\left[\gamma_{U}\right]+\gamma_{U}^{-1} \partial^{0} U S^{+}\left[\partial^{(2)} U\right]\right| & \leq K h\left[\left|\partial^{(2)} U\right|^{2}+T^{+}\left|\partial^{(2)} U\right|^{2}\right], \\
\left|\partial^{+}\left[\gamma_{U}^{-1}\right]-\gamma_{U}^{-3} \partial^{0} U S^{+}\left[\partial^{(2)} U\right]\right| & \leq K h\left[\left|\partial^{(2)} U\right|^{2}+T^{+}\left|\partial^{(2)} U\right|^{2}\right], \\
\left|\partial^{+}\left[\gamma_{U}^{-2}\right]-2 \gamma_{U}^{-4} \partial^{0} U S^{+}\left[\partial^{(2)} U\right]\right| & \leq K h\left[\left|\partial^{(2)} U\right|^{2}+T^{+}\left|\partial^{(2)} U\right|^{2}\right], \\
\left|\partial^{+}\left[\gamma_{U}^{-4}\right]-4 \gamma_{U}^{-6} \partial^{0} U S^{+}\left[\partial^{(2)} U\right]\right| & \leq K h\left[\left|\partial^{(2)} U\right|^{2}+T^{+}\left|\partial^{(2)} U\right|^{2}\right] .
\end{aligned}
$$


Proof Using the representation in Lemma C.2, we see that

$$
\left|\partial^{+} \gamma_{U}\right| \leq C_{1}^{\prime}\left[\left|\partial^{(2)} U\right|+T^{+}\left|\partial^{(2)} U\right|\right]
$$

together with

$$
\left|\partial^{+} \partial^{0} U\right|=\left|S^{+}\left[\partial^{(2)} U\right]\right| \leq\left|\partial^{(2)} U\right|+T^{+}\left|\partial^{(2)} U\right| .
$$

This implies that

$$
\begin{array}{r}
\left|S^{+}\left[\partial^{0} U\right]-\partial^{0} U\right| \leq C_{2}^{\prime} h\left[\left|\partial^{(2)} U\right|+T^{+}\left|\partial^{(2)} U\right|\right], \\
\left|S^{+}\left[\gamma_{U}\right]-\gamma_{U}\right|+\left|S^{+}\left[\gamma_{U}^{2}\right]-\gamma_{U}^{2}\right| \leq C_{2}^{\prime} h\left[\left|\partial^{(2)} U\right|+T^{+}\left|\partial^{(2)} U\right|\right], \\
\left|P^{+}\left[\gamma_{U}\right]-\gamma_{U}^{2}\right|+\left|P^{+}\left[\gamma_{U}^{2}\right]-\gamma_{U}^{4}\right| \leq C_{2}^{\prime} h\left[\left|\partial^{(2)} U\right|+T^{+}\left|\partial^{(2)} U\right|\right] .
\end{array}
$$

Since the explicit expressions on the left hand side in (D.5) can be obtained from Lemma C.2 by making the replacements

$$
S^{+}\left[\partial^{0} U\right] \mapsto \partial^{0} U, \quad S^{+} \gamma_{U} \mapsto \gamma_{U}, \quad S^{+} \gamma_{U}^{2} \mapsto \gamma_{U}^{2}, \quad P^{+} \gamma_{U} \mapsto \gamma_{U}^{2} \quad P^{+} \gamma_{U}^{2} \mapsto \gamma_{U}^{4},
$$

the desired estimates follow from the lower bounds for $\gamma_{U}$ stated in (D.1).

\section{D.2 Discrete Derivative Estimates}

In this subsection we obtain several preliminary estimates concerning the discrete derivatives introduced in $\$ 3.2$ and the mixed expressions $\gamma_{U}^{-k} \partial^{0} U$. We also consider an approximation for a sum that can be seen as the discrete version of the integral identity

$$
\int_{-\infty}^{\tau} \frac{u^{\prime}(\tilde{\tau}) u^{\prime \prime}(\tilde{\tau})}{1-u^{\prime}(\tilde{\tau})^{2}} d \tilde{\tau}=\frac{1}{2} \ln \left[1-u^{\prime}(\tau)^{2}\right] .
$$

Lemma D.5 Fix $h>0$ and $0<\kappa<\frac{1}{12}$. Then for any $U \in \Omega_{h ; \kappa}$, we have the inclusions

$$
\left\{\mathcal{D}^{\diamond^{ \pm}}(U), \mathcal{D}^{\diamond_{0}}(U), \mathcal{D}^{\diamond_{0}}(U), \mathcal{D}^{\diamond_{0} ;+}(U), \mathcal{D}^{\diamond ;} ;(U), \mathcal{D}^{\diamond_{0} ;+}(U)\right\} \subset \ell_{h}^{2} .
$$

Proof Proposition 3.1 implies that $\partial^{ \pm} U \in \ell_{h}^{2}$, Together with Lemma D.1 and the identity (3.17), this implies the inclusions

$$
\mathcal{D}^{\diamond \pm}(U) \in \ell_{h}^{2}, \quad \mathcal{D}^{\diamond}(U) \in \ell_{h}^{2} .
$$

Since $\partial^{ \pm}\left(\ell_{h}^{2}\right) \subset \ell_{h}^{2}$, the remaining inclusions can be read off from the definitions (3.21), (3.26) and (3.27).

Corollary D.6 Fix $0<\kappa<\frac{1}{12}$. Then there exists $K>0$ so that for any $h>0$ and any $U \in \Omega_{h ; \kappa}$, we have the pointwise bounds

$$
\begin{aligned}
& \left|\partial^{+} \mathcal{D}^{\diamond_{-}}(U)\right| \leq K\left|\partial^{(2)} U\right| \\
& \left|\partial^{+} \mathcal{D}^{\diamond_{0}}(U)\right| \leq K\left[\left|\partial^{(2)} U\right|+T^{+}\left|\partial^{(2)} U\right|\right] .
\end{aligned}
$$

Proof This follows directly from Lemma C.3. 
Lemma D.7 Fix $h>0$ and $0<\kappa<\frac{1}{12}$. Then for any $U \in \Omega_{h ; \kappa}$, we have the pointwise bounds

$$
\frac{1}{2} \kappa<\mathcal{D}^{\diamond 0}(U) \mathcal{D}^{\diamond}(U)+1<\frac{3}{2} \kappa^{-1}
$$

Proof We compute

$$
\begin{aligned}
\mathcal{D}^{\diamond_{0}}(U) \mathcal{D}^{\diamond}(U)+1 & =1+\frac{\partial^{+} U+\partial^{-} U}{r_{U}^{-}+r_{U}^{+}} \frac{\partial^{+} U}{r_{U}^{+}} \\
& =\frac{r_{U}^{+}\left(r_{U}^{-}+r_{U}^{+}\right)+\left(\partial^{+} U\right)^{2}+\partial^{-} U \partial^{+} U}{r_{U}^{+}\left(r_{U}^{-}+r_{U}^{+}\right)} .
\end{aligned}
$$

Since $\left(r_{U}^{+}\right)^{2}+\left(\partial^{+} U\right)^{2}=1$, we obtain

$$
\mathcal{D}^{\diamond_{0}}(U) \mathcal{D}^{\diamond}(U)+1=\frac{r_{U}^{+} r_{U}^{-}}{r_{U}^{+}\left(r_{U}^{-}+r_{U}^{+}\right)}+\frac{1+\partial^{-} U \partial^{+} U}{r_{U}^{+}\left(r_{U}^{-}+r_{U}^{+}\right)} .
$$

Observe that $\left|\partial^{-} U\right|\left|\partial^{+} U\right|<1$. In addition, Lemma D.1 implies

$$
2 \kappa<r_{U}^{+}\left(r_{U}^{-}+r_{U}^{+}\right) \leq 2, \quad \kappa<r_{U}^{+} r_{U}^{-} \leq 1 .
$$

We hence find

$$
\frac{\kappa}{2}<\mathcal{D}^{\diamond_{0}}(U) \mathcal{D}^{\diamond+}(U)+1<\frac{3}{2 \kappa},
$$

as desired. The estimate involving $\mathcal{D}^{\diamond}(U)$ can be obtained in the same fashion.

Lemma D.8 Fix $0<\kappa<\frac{1}{12}$. Then there exists $K>0$ so that for any $h>0$ and any $U \in \Omega_{h ; \kappa}$, we have the pointwise estimate

$$
\left|\partial^{+}\left[\frac{\partial^{0} U}{\gamma_{U}^{2}}\right]-\gamma_{U}^{-4}\left(2-\gamma_{U}^{2}\right) S^{+} \partial^{(2)} U\right| \leq K h\left[\left|\partial^{(2)} U\right|+T^{+}\left|\partial^{(2)} U\right|\right] .
$$

Proof Using $\partial^{+} \partial^{0} U=S^{+} \partial^{(2)} U$ and the definition (7.6) for $\gamma_{U}$, we compute

$$
\begin{aligned}
\partial^{+}\left[\frac{\partial^{0} U}{\gamma_{U}^{2}}\right] & =\partial^{+}\left[\gamma_{U}^{-2}\right] T^{+} \partial^{0} U+\gamma_{U}^{-2} \partial^{+} \partial^{0} U \\
& =\partial^{+}\left[\gamma_{U}^{-2}\right] \partial^{0} U+\mathcal{E}_{1}(U)+\gamma_{U}^{-2} \partial^{+} \partial^{0} U \\
& =2 \gamma_{U}^{-4} \partial^{0} U S^{+}\left[\partial^{(2)} U\right] \partial^{0} U+\mathcal{E}_{1}(U)+\mathcal{E}_{2}(U)+\gamma_{U}^{-2} S^{+}\left[\partial^{(2)} U\right] \\
& =\gamma_{U}^{-4}\left(2-\gamma_{U}^{2}\right) S^{+}\left[\partial^{(2)} U\right]+\mathcal{E}_{1}(U)+\mathcal{E}_{2}(U)
\end{aligned}
$$

in which

$$
\begin{aligned}
& \mathcal{E}_{1}(U)=h \partial^{+}\left[\gamma_{U}^{-2}\right] \partial^{+} \partial^{0} U \\
& \mathcal{E}_{2}(U)=\left[\partial^{+}\left[\gamma_{U}^{-2}\right]-2 \gamma_{U}^{-4} \partial^{0} U S^{+}\left[\partial^{(2)} U\right]\right] \partial^{0} U .
\end{aligned}
$$

The desired estimate now follows from the bounds (D.5). 
Lemma D.9 Fix $0<\kappa<\frac{1}{12}$. Then there exists $K>0$ so that for any $h>0$ and any $U \in \Omega_{h ; \kappa}$, we have the pointwise estimate

$$
\left|\sum_{-; h} \gamma_{U}^{-2} \partial^{0} U S^{+}\left[\partial^{(2)} U\right]+\ln \left[\gamma_{U}\right]\right| \leq K h .
$$

Proof We first compute

$$
\partial^{+}\left[\ln \gamma_{U}\right]=\frac{1}{h} \ln T^{+} \gamma_{U}-\frac{1}{h} \ln \gamma_{U}=\frac{1}{h} \ln \frac{T^{+} \gamma_{U}}{\gamma_{U}} .
$$

The bounds in Lemma D.1 imply that

$$
\frac{T^{+} \gamma_{U}}{\gamma_{U}} \geq \sqrt{\kappa}
$$

We recall that

$$
|\ln (1+x)-x| \leq C_{1}^{\prime}|x|^{2}
$$

holds for all $x \in \mathbb{R}$ that have $1+x \geq \sqrt{\kappa}>0$. Applying this estimate with

$$
x=\frac{T^{+} \gamma_{U}}{\gamma_{U}}-1=h \gamma_{U}^{-1} \partial^{+}\left[\gamma_{U}\right],
$$

we conclude that the sequence

$$
\mathcal{I}_{1}=\partial^{+}\left[\ln \gamma_{U}\right]-\gamma_{U}^{-1} \partial^{+}\left[\gamma_{U}\right]
$$

satisfies the pointwise bound

$$
\left|\mathcal{I}_{1}\right| \leq C_{1}^{\prime} h^{-1}\left[h \gamma_{U}^{-1}\left|\partial^{+}\left[\gamma_{U}\right]\right|\right]^{2} .
$$

Using the explicit expression for $\partial^{+}\left[\gamma_{U}\right]$ in Lemma C.2, we conclude

$$
\left|\mathcal{I}_{1}\right| \leq C_{2}^{\prime} h\left[\left|\partial^{(2)} U\right|^{2}+T^{+}\left|\partial^{(2)} U\right|^{2}\right] .
$$

Writing

$$
\mathcal{I}_{2}=\partial^{+}\left[\ln \gamma_{U}\right]+\gamma_{U}^{-2} \partial^{0} U S^{+}\left[\partial^{(2)} U\right],
$$

the estimate (D.5) implies that also

$$
\left|\mathcal{I}_{2}\right| \leq C_{3}^{\prime} h\left[\left|\partial^{(2)} U\right|^{2}+T^{+}\left|\partial^{(2)} U\right|^{2}\right] .
$$

In particular, we see that

$$
\begin{array}{r}
\left|\sum_{-; h} \mathcal{I}_{2}\right| \leq 2 C_{3}^{\prime} h, \\
\left\|\partial^{+} \partial^{+} U\right\|_{\ell_{h}^{2}}^{2} \leq C_{4}^{\prime} h .
\end{array}
$$

Since $\left[\gamma_{U}\right]_{j h} \rightarrow 1$ as $j \rightarrow-\infty$, we conclude that

$$
\ln \left[\gamma_{U}\right]=\sum_{-; h} \partial^{+}\left[\ln \gamma_{U}\right]
$$

must hold pointwise. The desired estimate follows directly from this identity and the bound (D.32). 


\section{E Approximate Substitution}

In this section we provide the framework that we use throughout the remainder of the appendices to establish the approximation results in $\S 7.1$. The setting is similar to that of $\S 5$, but now we consider composite functions $f \circ \phi$ in situations where it is convenient to approximate $\phi$ and $D \phi$ by $\phi_{\text {apx }}$ and $\phi_{\text {lin. }}$. These two approximants should be thought of as simplified versions of $\phi$ and $D \phi$ that are much easier to handle in computations, while still accurate to leading order in $h$. Our typical setup is described in the following assumption.

$(\mathrm{h} \phi)$ The set $K_{f} \subset \mathbb{R}^{n}$ is compact and we have the inclusion $\Omega_{\phi} \subset \mathcal{B}$, in which $\mathcal{B}$ is a Banach space. In addition, the function

$$
\phi: \Omega_{\phi} \subset \mathcal{B} \rightarrow K_{f}
$$

is Lipschitz continuous in the sense that there is $K_{\text {lip }}>1$ so that

$$
\left|\phi\left(\omega_{1}\right)-\phi\left(\omega_{2}\right)\right| \leq K_{\text {lip }}\left\|\omega_{1}-\omega_{2}\right\|_{\mathcal{B}}
$$

holds for all $\omega_{1}, \omega_{2} \in \Omega_{\phi}$. Finally, we have the inclusions

$$
\phi_{\text {apx }}(\omega) \in K_{f}, \quad \phi_{\text {lin } ; \omega} \in \mathcal{L}\left(\mathcal{B} ; \mathbb{R}^{n}\right)
$$

for every $\omega \in \Omega_{\phi}$, together with the uniform bound

$$
\sup _{\omega \in \Omega_{\phi}}\left\|\phi_{\operatorname{lin} ; \omega}\right\|_{\mathcal{L}\left(\mathcal{B} ; \mathbb{R}^{n}\right)}<\infty .
$$

Lemma E.1 Consider two triplets $\left(\phi, \phi_{\mathrm{apx}}, \phi_{\text {lin }}\right)$ and $\left(\Omega_{\phi}, \mathcal{B}, K_{f}\right)$ and suppose that $(h \phi)$ is satisfied. Suppose furthermore that there exists an open set $O_{f} \subset \mathbb{R}^{n}$ and a compact set $\bar{K}_{f} \subset \mathbb{R}^{n}$ for which

$$
K_{f} \subset O_{f} \subset \bar{K}_{f} .
$$

Pick any $f \in C^{2}\left(\bar{K}_{f} ; \mathbb{R}\right)$ and consider the map

$$
P: \Omega_{\phi} \subset \mathcal{B} \rightarrow \mathbb{R}, \quad \omega \mapsto f(\phi(\omega)) .
$$

For any $\omega \in \Omega_{\phi}$ and $\beta \in \mathcal{B}$, write

$$
\begin{aligned}
P_{\text {apx }}(\omega) & =f\left(\phi_{\text {apx }}(\omega)\right), \\
P_{\text {lin } ; \omega}[\beta] & =D f\left(\phi_{\text {apx }}(\omega)\right) \phi_{\operatorname{lin} ; \omega}[\beta] .
\end{aligned}
$$

In addition, for any $\omega \in \Omega_{\phi}$ and $\beta \in \mathcal{B}$ for which $\omega+\beta \in \Omega_{\phi}$, write

$$
\begin{aligned}
& \phi_{\mathrm{nl} ; \omega}(\beta)=\phi(\omega+\beta)-\phi(\omega)-\phi_{\operatorname{lin} ; \omega}[\beta], \\
& P_{\mathrm{nl} ; \omega}(\beta)=P(\omega+\beta)-P(\omega)-P_{\operatorname{lin} ; \omega}[\beta] .
\end{aligned}
$$

Then there exists a constant $K>0$ so that for any $\omega \in \Omega_{\phi}$ the bound

$$
\left|P(\omega)-P_{\text {apx }}(\omega)\right| \leq K\left|\phi(\omega)-\phi_{\text {apx }}(\omega)\right|
$$

holds, while for any $\omega \in \Omega_{\phi}$ and $\beta \in \mathcal{B}$ for which $\omega+\beta \in \Omega_{\phi}$ we have the estimate

$$
\left|P_{\mathrm{nl} ; \omega}(\beta)\right| \leq K\left[\|\beta\|_{\mathcal{B}}^{2}+\left|\phi_{\mathrm{nl} ; \omega}(\beta)\right|+\left|\phi(\omega)-\phi_{\mathrm{apx}}(\omega)\right|\|\beta\|_{\mathcal{B}}\right] .
$$


Proof The geometric condition (E.5) implies that $f$ and $D f$ are Lipschitz on $K_{f}$ and that there is $C_{1}>0$ for which

$$
\frac{|f(y)-f(x)-D f(x)(y-x)|}{|y-x|^{2}} \leq C_{1}
$$

holds for all $(x, y) \in K_{f} \times K_{f}$ with $x \neq y$. Indeed, we can cover $K_{f}$ completely with open balls in which the local versions of these properties follow from the $C^{2}$-smoothness of $f$ on the larger set $\bar{K}_{f}$.

The inequality (E.9) follows directly from the fact that $f$ is Lipschitz. Turning to (E.10), we decompose

$$
P_{\mathrm{nl} ; \omega}(\beta)=\mathcal{J}_{1}+\mathcal{J}_{2}+\mathcal{J}_{3}
$$

in which

$$
\begin{aligned}
& \mathcal{J}_{1}=f(\phi(\omega+\beta))-f(\phi(\omega))-D f(\phi(\omega))[\phi(\omega+\beta)-\phi(\omega)], \\
& \mathcal{J}_{2}=D f(\phi(\omega)) \phi_{\mathrm{nl} ; \omega}(\beta), \\
& \mathcal{J}_{3}=\left[D f(\phi(\omega))-D f\left(\phi_{\mathrm{apx}}(\omega)\right)\right] \phi_{\operatorname{lin} ; \omega}[\beta] .
\end{aligned}
$$

The bounds (E.2) and (E.11) imply

$$
\left|\mathcal{J}_{1}\right| \leq C_{1}|\phi(\omega+\beta)-\phi(\omega)|^{2} \leq C_{1} K_{\text {lip }}^{2}\|\beta\|_{\mathcal{B}}^{2},
$$

while the Lipschitz smoothness of $D f$ yields

$$
\left|\mathcal{J}_{3}\right| \leq C_{2}\left|\phi(\omega)-\phi_{\text {apx }}(\omega)\right|\left\|\phi_{\text {lin; } \omega}\right\|_{\mathcal{L}\left(\mathcal{B} ; \mathbb{R}^{n}\right)}\|\beta\|_{\mathcal{B}}
$$

for some $C_{2}>0$. The desired estimate (E.10) now follows from the uniform bound (E.4).

Corollary E.2 Consider two triplets $\left(\phi, \phi_{\mathrm{apx}}, \phi_{\mathrm{lin}}\right)$ and $\left(\Omega_{\phi}, \mathcal{B}, K_{f}\right)$ and suppose that $(h \phi)$ is satisfied. Suppose furthermore that there exists an open set $O_{f} \subset \mathbb{R}^{n}$ and a compact set $\bar{K}_{f} \subset \mathbb{R}^{n}$ for which

$$
K_{f} \subset O_{f} \subset \bar{K}_{f} .
$$

Pick any $f \in C^{2}\left(\bar{K}_{f} ; \mathbb{R}\right)$, any Banach space $\mathcal{B}_{L}$, any $L \in \mathcal{L}\left(\mathcal{B}_{L} ; \mathbb{R}\right)$ and consider the map

$$
P: \Omega_{\phi} \times \mathcal{B}_{L} \rightarrow \mathbb{R}, \quad\left(\omega, \omega_{L}\right) \mapsto f(\phi(\omega)) L\left[\omega_{L}\right] .
$$

For any $\left(\omega, \omega_{L}\right) \in \Omega_{\phi} \times \mathcal{B}_{L}$ and $\left(\beta, \beta_{L}\right) \in \mathcal{B} \times \mathcal{B}_{L}$, write

$$
\begin{aligned}
P_{\text {apx }}\left(\omega, \omega_{L}\right) & =f\left(\phi_{\text {apx }}(\omega)\right) L\left[\omega_{L}\right], \\
P_{\operatorname{lin} ; \omega, \omega_{L}}\left[\beta, \beta_{L}\right] & =D f\left(\phi_{\text {apx }}(\omega)\right) L\left[\omega_{L}\right] \phi_{\text {lin } ; \omega}[\beta]+f\left(\phi_{\text {apx }}(\omega)\right) L\left[\beta_{L}\right] .
\end{aligned}
$$

In addition, for any $\left(\omega, \omega_{L}\right) \in \Omega_{\phi} \times \mathcal{B}_{L}$ and $\left(\beta, \beta_{L}\right) \in \mathcal{B} \times \mathcal{B}_{L}$ for which $\omega+\beta \in \Omega_{\phi}$, write

$$
P_{\mathrm{nl} ; \omega, \omega_{L}}\left(\beta, \beta_{L}\right)=P\left(\omega+\beta, \omega_{L}+\beta_{L}\right)-P\left(\omega, \omega_{L}\right)-P_{\operatorname{lin} ; \omega, \omega_{L}}\left[\beta, \beta_{L}\right] .
$$

Then there exists a constant $K>0$ so that for any $\left(\omega, \omega_{L}\right) \in \Omega_{\phi} \times \mathcal{B}_{L}$ we have the bound

$$
\left|P\left(\omega, \omega_{L}\right)-P_{\text {apx }}\left(\omega, \omega_{L}\right)\right| \leq K\left|\phi(\omega)-\phi_{\text {apx }}(\omega)\right|\left\|\omega_{L}\right\|_{\mathcal{B}_{L}},
$$

while for any for any $\left(\omega, \omega_{L}\right) \in \Omega_{\phi} \times \mathcal{B}_{L}$ and $\left(\beta, \beta_{L}\right) \in \mathcal{B} \times \mathcal{B}_{L}$ for which $\omega+\beta \in \Omega_{\phi}$ we have the bound

$$
\begin{aligned}
\left|P_{\mathrm{nl} ; \omega, \omega_{L}}\left(\beta, \beta_{L}\right)\right| \leq & K\left[\|\beta\|_{\mathcal{B}}^{2}\left\|\omega_{L}\right\|_{\mathcal{B}_{L}}+\|\beta\|_{\mathcal{B}}\left\|\beta_{L}\right\|_{\mathcal{B}_{L}}+\left|\phi_{\mathrm{nl} ; \omega}(\beta)\right|\left\|\omega_{L}\right\|_{\mathcal{B}_{L}}\right. \\
& \left.+\left|\phi(\omega)-\phi_{\mathrm{apx}}(\omega)\right|\left[\|\beta\|_{\mathcal{B}}\left\|\omega_{L}\right\|_{\mathcal{B}_{L}}+\left\|\beta_{L}\right\|_{\mathcal{B}_{L}}\right]\right] .
\end{aligned}
$$


Proof The bound (E.20) follows immediately from (E.9) together with the fact that $L \in$ $\mathcal{L}\left(\mathcal{B}_{L} ; \mathbb{R}\right)$. Upon writing $P\left(\omega, \omega_{L}\right)=\widetilde{P}(\omega) L\left[\omega_{L}\right]$, we see that

$$
\begin{aligned}
P_{\mathrm{nl} ; \omega, \omega_{L}}\left(\beta, \beta_{L}\right)= & \widetilde{P}_{\mathrm{nl} ; \omega}(\beta) L\left[\omega_{L}\right]+\left[f(\phi(\omega+\beta))-f\left(\phi_{\mathrm{apx}}(\omega)\right)\right] L\left[\beta_{L}\right] \\
= & \widetilde{P}_{\mathrm{nl} ; \omega}(\beta) L\left[\omega_{L}\right]+[f(\phi(\omega+\beta))-f(\phi(\omega))] L\left[\beta_{L}\right] \\
& +\left[f(\phi(\omega))-f\left(\phi_{\mathrm{apx}}(\omega)\right)\right] L\left[\beta_{L}\right] .
\end{aligned}
$$

In particular, exploiting the Lipschitz continuity of $f$ and $\phi$, we can find a constant $C_{1}>0$ for which

$$
\begin{aligned}
\left|P_{\mathrm{nl} ; \omega, \omega_{L}}\left(\beta, \beta_{L}\right)\right| \leq C_{1} \mid & \widetilde{P}_{\mathrm{nl} ; \omega}(\beta) \mid\left\|\omega_{L}\right\|_{\mathcal{B}_{L}}+C_{1}\|\beta\|_{\mathcal{B}}\left\|\beta_{L}\right\|_{\mathcal{B}_{L}} \\
& +C_{1}\left|\phi(\omega)-\phi_{\mathrm{apx}}(\omega)\right|\left\|\beta_{L}\right\|_{\mathcal{B}_{L}} .
\end{aligned}
$$

Substituting the estimate (E.10) for $\widetilde{P}_{\mathrm{nl} ; \omega}(\beta)$ yields the desired bound (E.21).

\section{F Component Estimates I}

In this section we establish the technical estimates that were summarized in Propositions 7.1, $7.2,7.3$ and 7.5. The main idea is to apply the substitution techniques from $\S \mathrm{E}$ to the explicit identities derived in $\S 3$. This leads to a large number of tedious but relatively straightforward calculations.

\section{F.1 Gridpoint Spacing}

Recalling (3.16), we define the approximate derivative

$$
r_{\operatorname{lin} ; U}[V]=-\gamma_{U}^{-1} \partial^{0} U \partial^{0} V
$$

together with the nonlinear residuals

$$
\begin{aligned}
& r_{\mathrm{nl} ; U}^{ \pm}(V)=r_{U+V}^{ \pm}-r_{U}^{ \pm}-r_{\operatorname{lin} ; U}[V], \\
& r_{\mathrm{nl} ; U}^{0}(V)=r_{U+V}^{0}-r_{U}^{0}-r_{\operatorname{lin} ; U}[V] .
\end{aligned}
$$

Lemma F.1 Fix $0<\kappa<\frac{1}{12}$. Then there exists $K>0$ so that the pointwise bounds

$$
\begin{aligned}
\left|r_{U}^{0}-\gamma_{U}\right|+\left|r_{U}^{+}-\gamma_{U}\right|+\left|r_{U}^{-}-\gamma_{U}\right| \leq & K h\left|\partial^{(2)} U\right| \\
\left|r_{\mathrm{nl} ; U}^{0}(V)\right|+\left|r_{\mathrm{nl} ; U}^{+}(V)\right|+\left|r_{\mathrm{nl} ; U}^{+}(V)\right| \leq & K\left[\left|\partial^{+} V\right|^{2}+\left|\partial^{-} V\right|^{2}\right] \\
& +K h\left[\left|\partial^{+} V\right|+\left|\partial^{-} V\right|+\left|\partial^{(2)} V\right|\right]
\end{aligned}
$$

hold for any $h>0$, any $U \in \Omega_{h ; \kappa}$ and any $V \in \ell_{h}^{2}$ for which $U+V \in \Omega_{h ; \kappa}$.

Proof We consider only the statements concerning $r_{U}^{+}$as the functions $r_{U}^{-}$and $r_{U}^{0}$ can be treated in a similar fashion. Writing $f(x)=\sqrt{1-x^{2}}$ and $\phi\left(\partial^{-} U, \partial^{+} U\right)=\partial^{+} U$, we see that

$$
r_{U}^{+}=f\left(\phi\left(\partial^{-} U, \partial^{+} U\right)\right) .
$$


We include the redundant variable $\partial^{-} U$ here because it will be used for our approximate function

$$
\phi_{\text {apx }}\left(\partial^{-} U, \partial^{+} U\right)=\frac{1}{2} \partial^{+} U+\frac{1}{2} \partial^{-}(U)=\partial^{0} U
$$

and our approximate derivative

$$
\phi_{\operatorname{lin} ; U}\left[\partial^{-} V, \partial^{+} V\right]=\partial^{0} V .
$$

A short computation shows that

$$
\phi\left(\partial^{-} U, \partial^{+} U\right)-\phi_{\text {apx }}\left(\partial^{-} U, \partial^{+} U\right)=\partial^{+} U-\partial^{0} U=\frac{1}{2} h \partial^{(2)} U,
$$

together with

$$
\begin{aligned}
\phi_{\mathrm{nl} ; U}\left(\partial^{-} V, \partial^{+} V\right) & =\partial^{+}(U+V)-\partial^{+} U-\partial^{0} V \\
& =\partial^{+} V-\partial^{0} V \\
& =\frac{1}{2} h \partial^{(2)} V .
\end{aligned}
$$

The a-priori estimate (3.42) ensures that the geometric condition (E.5) can be satisfied. In particular, the bounds now follow directly from Lemma E.1 and the observations

$$
\begin{aligned}
f\left(\phi_{\text {apx }}\left(\partial^{-} U, \partial^{+} U\right)\right) & =\gamma_{U}, \\
D f\left(\phi_{\text {apx }}\left(\partial^{-} U, \partial^{+} U\right)\right) & =-\gamma_{U}^{-1} \partial^{0} U .
\end{aligned}
$$

\section{F.2 First Order Discrete Derivatives}

Recalling (3.17), we write

$$
\begin{aligned}
& \mathcal{D}_{\text {apx }}^{\diamond_{+}}(U)=\mathcal{D}_{\text {apx }}^{\diamond_{-}}(U)=\mathcal{D}_{\text {apx }}^{\diamond_{0}}(U)=\gamma_{U}^{-1} \partial^{0} U, \\
& \mathcal{D}_{\text {lin; } U}^{\diamond+}[V]=\mathcal{D}_{\text {lin; } U}^{\diamond}[V]=\mathcal{D}_{\operatorname{lin} ; U}^{\diamond_{0}}[V]=\gamma_{U}^{-3} \partial^{0} V
\end{aligned}
$$

and introduce the nonlinear residuals

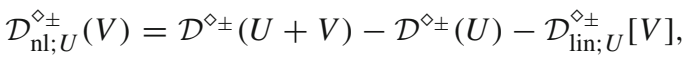

$$
\begin{aligned}
& \mathcal{D}_{\mathrm{nl} ; U}^{\diamond_{0}}(V)=\mathcal{D}^{\diamond_{0}}(U+V)-\mathcal{D}^{\diamond_{0}}(U)-\mathcal{D}_{\operatorname{lin} ; U}^{\diamond_{0}}[V] \text {. }
\end{aligned}
$$

Lemma F.2 Fix $0<\kappa<\frac{1}{12}$. Then there exists $K>0$ so that the pointwise bounds

$$
\left|\mathcal{D}^{\diamond_{0}}(U)-\mathcal{D}_{\mathrm{apx}}^{\diamond_{0}}(U)\right|+\left|\mathcal{D}^{\diamond+}(U)-\mathcal{D}_{\mathrm{apx}}^{\diamond_{+}}(U)\right|+\left|\mathcal{D}^{\diamond_{-}}(U)-\mathcal{D}_{\mathrm{apx}}^{\diamond_{-}}(U)\right| \leq K h\left|\partial^{(2)} U\right|
$$

and

$$
\begin{aligned}
\left|\mathcal{D}_{\mathrm{nl} ; U}^{\diamond_{0}}(V)\right|+\left|\mathcal{D}_{\mathrm{nl} ; U}^{\diamond_{+}}(V)\right|+\left|\mathcal{D}_{\mathrm{nl} ; U}^{\diamond_{-}}(V)\right| \leq & K\left[\left|\partial^{-} V\right|^{2}+\left|\partial^{+} V\right|^{2}\right] \\
& +K h\left[\left|\partial^{-} V\right|+\left|\partial^{+} V\right|+\left|\partial^{(2)} V\right|\right]
\end{aligned}
$$

hold for any $h>0$, any $U \in \Omega_{h ; \kappa}$ and any $V \in \ell_{h}^{2}$ for which $U+V \in \Omega_{h ; \kappa}$. 
Proof We consider only the statements concerning $\mathcal{D}^{\diamond}+$ as the functions $\mathcal{D}^{\diamond}$ and $\mathcal{D}^{\diamond}$ can be treated in a similar fashion. Recalling the fact that $r_{U}^{+}$depends only on $\partial^{+} U$, we abuse notation slightly to write

$$
\phi\left(\partial^{-} U, \partial^{+} U\right)=\left(\partial^{+} U, r_{U}^{+}\right) .
$$

Upon introducing $f(x, y)=x / y$, we see that

$$
\mathcal{D}^{\diamond}(U)=f\left(\phi\left(\partial^{-} U, \partial^{+} U\right)\right) .
$$

We now define the approximants

$$
\begin{aligned}
\phi_{\text {apx }}\left(\partial^{-} U, \partial^{+} U\right) & =\left(\partial^{0} U, \gamma_{U}\right), \\
\phi_{\operatorname{lin} ; U}\left[\partial^{-} V, \partial^{+} V\right] & =\left(\partial^{0} V,-\gamma_{U}^{-1} \partial^{0} U \partial^{0} V\right)
\end{aligned}
$$

and compute

$$
\begin{aligned}
\phi\left(\partial^{-} U, \partial^{+} U\right)-\phi_{\text {apx }}\left(\partial^{-} U, \partial^{+} U\right) & =\left(\partial^{+} U-\partial^{0} U, r_{U}^{+}-\gamma_{U}\right) \\
& =\left(\frac{h}{2} \partial^{(2)} U, r_{U}^{+}-\gamma_{U}\right)
\end{aligned}
$$

together with

$$
\begin{aligned}
\phi_{\mathrm{nl} ; U}\left(\partial^{-} V, \partial^{+} V\right) & =\left(\partial^{+} V-\partial^{0} V, r_{\mathrm{nl} ; U}^{+}(V)\right) \\
& =\left(\frac{h}{2} \partial^{(2)} V, r_{\mathrm{nl} ; U}^{+}(V)\right) .
\end{aligned}
$$

In particular, Lemma F.1 provides the bound

$$
\left|\phi\left(\partial^{-} U, \partial^{+} U\right)-\phi_{\text {apx }}\left(\partial^{-} U, \partial^{+} U\right)\right| \leq C_{1}^{\prime} h\left|\partial^{(2)} U\right|
$$

together with

$$
\left|\phi_{\mathrm{nl} ; U}\left(\partial^{-} V, \partial^{+} V\right)\right| \leq C_{1}^{\prime}\left[\left|\partial^{-} V\right|^{2}+\left|\partial^{+} V\right|^{2}\right]+C_{1}^{\prime} h\left[\left|\partial^{-} V\right|+\left|\partial^{+} V\right|+\left|\partial^{(2)} V\right|\right] .
$$

Upon computing

$$
\begin{aligned}
f\left(\phi_{\text {apx }}\left(\partial^{-} U, \partial^{+} U\right)\right) & =\gamma_{U}^{-1} \partial^{0} U, \\
D f\left(\phi_{\text {apx }}\left(\partial^{-} U, \partial^{+} U\right)\right) \phi_{\operatorname{lin} ; U}\left[\partial^{-} V, \partial^{+} V\right] & =\gamma_{U}^{-1} \partial^{0} V-\partial^{0} U \gamma_{U}^{-2}\left(-\gamma_{U}^{-1} \partial^{0} U \partial^{0} V\right) \\
& =\left[\gamma_{U}^{-1}+\left(\partial^{0} U\right)^{2} \gamma_{U}^{-3}\right] \partial^{0} V \\
& =\gamma_{U}^{-3} \partial^{0} V,
\end{aligned}
$$

the desired bounds follow directly from Lemma E.1.

Proof of Proposition 7.1 The results follow directly from Lemma F.2.

\section{F.3 Second Order Discrete Derivatives}

Turning to second derivatives, we recall (3.21) together with the definitions

$$
\begin{aligned}
& \mathcal{D}_{\mathrm{apx}}^{\diamond_{-} ;+}(U)=\gamma_{U}^{-3} \partial^{(2)} U, \\
& \mathcal{D}_{\text {lin; } ;}^{\diamond_{-} ;+}[V]=3 \gamma_{U}^{-5} \partial^{0} U\left[\partial^{(2)} U\right] \partial^{0} V+\gamma_{U}^{-3} \partial^{(2)} V
\end{aligned}
$$


and write

$$
\mathcal{D}_{\mathrm{nl} ; U}^{\diamond ;+}(V)=\mathcal{D}_{--;+}^{\diamond^{-}}(U+V)-\mathcal{D}^{\diamond-;+}(U)-\mathcal{D}_{\text {lin; } ;}^{\diamond ;+}[V]
$$

Lemma F.3 Fix $0<\kappa<\frac{1}{12}$. Then there exists $K>0$ so that the pointwise approximation estimate

$$
\left|\mathcal{D}^{\diamond-;+}(U)-\mathcal{D}_{\text {apx }}^{\diamond_{-} ;+}(U)\right| \leq K h\left|\partial^{(2)} U\right|
$$

and the residual bound

$$
\begin{aligned}
\left|\mathcal{D}_{\mathrm{nl} ; U}^{\diamond ;+}(V)\right| \leq & K\left[\left|\partial^{-} V\right|^{2}+\left|\partial^{+} V\right|^{2}+\left|\partial^{-} V\right|\left|\partial^{(2)} V\right|+\left|\partial^{+} V\right|\left|\partial^{(2)} V\right|\right] \\
& +K h\left[\left|\partial^{-} V\right|+\left|\partial^{+} V\right|+\left|\partial^{(2)} V\right|\right]
\end{aligned}
$$

both hold for any $h>0$, any $U \in \Omega_{h ; \kappa}$ and any $V \in \ell_{h}^{2}$ for which $U+V \in \Omega_{h ; \kappa}$.

Proof Motivated by the identity

$$
\mathcal{D}^{\diamond-;+}(U)=\frac{1}{r_{U}^{+}}\left[1+\mathcal{D}^{\diamond-}(U) \mathcal{D}^{\diamond_{0}}(U)\right] \partial^{(2)} U
$$

derived in Lemma C.3, we write

$$
f\left(y, z_{-}, z_{0}\right)=\frac{1}{y}\left[1+z_{-} z_{0}\right]
$$

together with

$$
\phi\left(\partial^{-} U, \partial^{+} U\right)=\left(r_{U}^{+}, \mathcal{D}^{\diamond}(U), \mathcal{D}^{\diamond}(U)\right)
$$

and finally

$$
P\left(\partial^{-} U, \partial^{+} U, \partial^{(2)} U\right)=f\left(\phi\left(\partial^{-} U, \partial^{+} U\right)\right) \partial^{(2)} U .
$$

This allows us to verify that

$$
\mathcal{D}^{\diamond ;+}(U)=P\left(\partial^{-} U, \partial^{+} U, \partial^{(2)} U\right) .
$$

We now define the approximants

$$
\begin{aligned}
\phi_{\text {apx }}\left(\partial^{-} U, \partial^{+} U\right) & =\left(\gamma_{U}, \gamma_{U}^{-1} \partial^{0} U, \gamma_{U}^{-1} \partial^{0} U\right), \\
\phi_{\operatorname{lin} ; U}\left[\partial^{-} V, \partial^{+} V\right] & =\left(-\gamma_{U}^{-1}\left[\partial^{0} U\right] \partial^{0} V, \gamma_{U}^{-3} \partial^{0} V, \gamma_{U}^{-3} \partial^{0} V\right)
\end{aligned}
$$

and compute

$\phi\left(\partial^{-} U, \partial^{+} U\right)-\phi_{\text {apx }}\left(\partial^{-} U, \partial^{+} U\right)=\left(r_{U}^{+}-\gamma_{U}^{-1}, \mathcal{D}^{\diamond}(U)-\mathcal{D}_{\text {apx }}^{\diamond}(U), \mathcal{D}^{\diamond}(U)-\mathcal{D}_{\text {apx }}^{\diamond}(U)\right)$

together with

$$
\phi_{\mathrm{nl} ; U}\left(\partial^{-} V, \partial^{+} V\right)=\left(r_{\mathrm{nl} ; U}^{+}(V), \mathcal{D}_{\mathrm{nl} ; U}^{\diamond_{-}}(V), \mathcal{D}_{\mathrm{nl} ; U}^{\diamond_{0}}(V)\right) .
$$

In particular, Lemma's F.1 and F.2 provide the bound

$$
\left|\phi\left(\partial^{-} U, \partial^{+} U\right)-\phi_{\text {apx }}\left(\partial^{-} U, \partial^{+} U\right)\right| \leq C_{1}^{\prime} h\left|\partial^{(2)} U\right|,
$$


together with

$$
\begin{aligned}
\left|\phi_{\mathrm{nl} ; U}\left(\partial^{-} V, \partial^{+} V\right)\right| \leq & C_{1}^{\prime}\left[\left|\partial^{-} V\right|^{2}+\left|\partial^{+} V\right|^{2}\right] \\
& +C_{1}^{\prime} h\left[\left|\partial^{-} V\right|+\left|\partial^{+} V\right|+\left|\partial^{(2)} V\right|\right] .
\end{aligned}
$$

Introducing the compressed nonlinearity

$$
\bar{f}(y, z)=f(y, z, z)=\frac{1}{y}\left(1+z^{2}\right)
$$

together with the compressed approximants

$$
\begin{aligned}
\bar{\phi}_{\mathrm{apx}}\left(\partial^{-} U, \partial^{+} U\right) & =\left(\gamma_{U}, \gamma_{U}^{-1} \partial^{0} U\right), \\
\bar{\phi}_{\operatorname{lin} ; U}\left[\partial^{-} V, \partial^{+} V\right] & =\left(-\gamma_{U}^{-1} \partial^{0} U \partial^{0} V, \gamma_{U}^{-3} \partial^{0} V\right),
\end{aligned}
$$

we see that

$$
\begin{aligned}
f\left(\phi_{\text {apx }}\left(\partial^{-} U, \partial^{+} U\right)\right) & =\bar{f}\left(\bar{\phi}_{\text {apx }}\left(\partial^{-} U, \partial^{+} U\right)\right), \\
D f\left(\phi_{\text {apx }}\left(\partial^{-} U, \partial^{+} U\right)\right) \phi_{\operatorname{lin} ; U}\left[\partial^{-} V, \partial^{+} V\right] & =D \bar{f}\left(\bar{\phi}_{\text {apx }}\left(\partial^{-} U, \partial^{+} U\right)\right) \bar{\phi}_{\operatorname{lin} ; U}\left[\partial^{-} V, \partial^{+} V\right] .
\end{aligned}
$$

Upon computing

$$
D \bar{f}(y, z)=\left(-\frac{1}{y^{2}}\left(1+z^{2}\right), 2 \frac{z}{y}\right),
$$

we hence see that the functions defined in (E.18) satisfy

$$
P_{\text {apx }}(U)=\gamma_{U}^{-1}\left(1+\left(\partial^{0} U\right)^{2} \gamma_{U}^{-2}\right) \partial^{(2)} U=\gamma_{U}^{-3} \partial^{(2)} U,
$$

together with

$$
\begin{aligned}
P_{\text {lin; } U}[V]= & -\gamma_{U}^{-2}\left(1+\left(\partial^{0} U\right)^{2} \gamma_{U}^{-2}\right)\left(-\gamma_{U}^{-1} \partial^{0} U\right) \partial^{0} V\left(\partial^{(2)} U\right) \\
& +2 \gamma_{U}^{-2} \partial^{0} U\left(\gamma_{U}^{-3} \partial^{0} V\right)\left(\partial^{(2)} U\right) \\
& +\gamma_{U}^{-1}\left(1+\left(\partial^{0} U\right)^{2} \gamma_{U}^{-2}\right) \partial^{(2)} V \\
= & 3 \gamma_{U}^{-5} \partial^{0} U\left[\partial^{(2)} U\right] \partial^{0} V+\gamma_{U}^{-3} \partial^{(2)} V .
\end{aligned}
$$

The desired estimates now follow directly from Corollary E.2.

We also recall (3.26), together with the definitions

$$
\begin{aligned}
\mathcal{D}_{\mathrm{apx}}^{\diamond \diamond_{0}}(U) & =\gamma_{U}^{-4} \partial^{(2)} U, \\
\mathcal{D}_{\operatorname{lin} ; U}^{\diamond{ }_{0}}[V] & =4 \gamma_{U}^{-6} \partial^{0} U\left[\partial^{(2)} U\right] \partial^{0} V+\gamma_{U}^{-4} \partial^{(2)} V
\end{aligned}
$$

and write

$$
\mathcal{D}_{\mathrm{nl} ; U}^{\diamond_{0}}(V)=\mathcal{D}^{\diamond_{0}}(U+V)-\mathcal{D}^{\diamond_{0}}(U)-\mathcal{D}_{\text {lin; } ;}^{\diamond_{0}}[V]
$$

Lemma F.4 Fix $0<\kappa<\frac{1}{12}$. Then there exists $K>0$ so that the pointwise approximation estimate

$$
\left|\mathcal{D}^{\diamond_{0}}(U)-\mathcal{D}_{\mathrm{apx}}^{\diamond_{0}}(U)\right| \leq K h\left|\partial^{(2)} U\right|
$$


and the residual bound

$$
\begin{aligned}
\left|\mathcal{D}_{\mathrm{nl} ; U}^{\diamond \diamond_{0}}(V)\right| \leq & K\left[\left|\partial^{-} V\right|^{2}+\left|\partial^{+} V\right|^{2}+\left|\partial^{-} V\right|\left|\partial^{(2)} V\right|+\left|\partial^{+} V\right|\left|\partial^{(2)} V\right|\right] \\
& +K h\left[\left|\partial^{-} V\right|+\left|\partial^{+} V\right|+\left|\partial^{(2)} V\right|\right]
\end{aligned}
$$

both hold for any $h>0$, any $U \in \Omega_{h ; \kappa}$ and any $V \in \ell_{h}^{2}$ for which $U+V \in \Omega_{h ; \kappa}$.

Proof Motivated by the identity

$$
\mathcal{D}^{\diamond_{0}}(U)=\frac{1}{r_{U}^{+} r_{U}^{0}}\left[1+\mathcal{D}^{\diamond}(U) \mathcal{D}^{\diamond_{0}}(U)\right] \partial^{(2)} U
$$

derived in Lemma C.3 and (3.26), we write

$$
f\left(y_{+}, y_{0}, z_{-}, z_{0}\right)=\frac{1}{y_{+} y_{0}}\left[1+z_{-} z_{0}\right]
$$

together with

$$
\phi\left(\partial^{-} U, \partial^{+} U\right)=\left(r_{U}^{+}, r_{U}^{0}, \mathcal{D}^{\diamond-}(U), \mathcal{D}^{\diamond}(U)\right)
$$

and finally

$$
P\left(\partial^{-} U, \partial^{+} U, \partial^{(2)} U\right)=f\left(\phi\left(\partial^{-} U, \partial^{+} U\right)\right) \partial^{(2)} U .
$$

This allows one to verify that

$$
\mathcal{D}^{\diamond \diamond_{0}}(U)=P\left(\partial^{-} U, \partial^{+} U, \partial^{(2)} U\right) .
$$

We now define the approximants

$$
\begin{aligned}
\phi_{\text {apx }}\left(\partial^{-} U, \partial^{+} U\right) & =\left(\gamma_{U}, \gamma_{U}, \gamma_{U}^{-1} \partial^{0} U, \gamma_{U}^{-1} \partial^{0} U\right), \\
\phi_{\operatorname{lin} ; U}\left[\partial^{-} V, \partial^{+} V\right] & =\left(-\gamma_{U}^{-1}\left[\partial^{0} U\right] \partial^{0} V,-\gamma_{U}^{-1}\left[\partial^{0} U\right] \partial^{0} V, \gamma_{U}^{-3} \partial^{0} V, \gamma_{U}^{-3} \partial^{0} V\right) .
\end{aligned}
$$

This allows us to compute

$$
\begin{aligned}
& \phi\left(\partial^{-} U, \partial^{+} U\right)-\phi_{\text {apx }}\left(\partial^{-} U, \partial^{+} U\right) \\
& =\left(r_{U}^{+}-\gamma_{U}^{-1}, r_{U}^{0}-\gamma_{U}^{-1}, \mathcal{D}^{\diamond}(U)-\mathcal{D}_{\text {apx }}^{\diamond}, \mathcal{D}^{\diamond}(U)-\mathcal{D}_{\text {apx }}^{\diamond}\right),
\end{aligned}
$$

together with

$$
\phi_{\mathrm{nl} ; U}\left(\partial^{-} V, \partial^{+} V\right)=\left(r_{\mathrm{nl} ; U}^{+}(V), r_{\mathrm{nl} ; U}^{0}(V) \mathcal{D}_{\mathrm{nl} ; U}^{\diamond_{-}}(V), \mathcal{D}_{\mathrm{nl} ; U}^{\diamond_{0}}(V)\right) .
$$

In particular, the bounds (F.34)-(F.35) remain valid.

This allows us to repeat the procedure in the proof of Lemma F.3 with the compressed approximants (F.37) and the compressed nonlinearity

$$
\bar{f}(y, z)=f(y, y, z, z)=\frac{1}{y^{2}}\left(1+z^{2}\right),
$$

for which we have

$$
D \bar{f}(y, z)=\left(-\frac{2}{y^{3}}\left(1+z^{2}\right), 2 \frac{z}{y^{2}}\right)
$$


The functions defined in (E.18) hence satisfy

$$
P_{\text {apx }}(U)=\gamma_{U}^{-2}\left(1+\left(\partial^{0} U\right)^{2} \gamma_{U}^{-2}\right) \partial^{(2)} U=\gamma_{U}^{-4} \partial^{(2)} U,
$$

together with

$$
\begin{aligned}
P_{\text {lin } ;}[V]= & -2 \gamma_{U}^{-3}\left(1+\left(\partial^{0} U\right)^{2} \gamma_{U}^{-2}\right)\left(-\gamma_{U}^{-1} \partial^{0} U\right) \partial^{0} V\left(\partial^{(2)} U\right) \\
& +2 \gamma_{U}^{-3} \partial^{0} U\left(\gamma_{U}^{-3} \partial^{0} V\right)\left(\partial^{(2)} U\right) \\
& +\gamma_{U}^{-2}\left(1+\left(\partial^{0} U\right)^{2} \gamma_{U}^{-2}\right) \partial^{(2)} V \\
= & 4 \gamma_{U}^{-6} \partial^{0} U\left[\partial^{(2)} U\right] \partial^{0} V+\gamma_{U}^{-4} \partial^{(2)} V .
\end{aligned}
$$

The desired estimates again follow directly from Corollary E.2.

Finally, we recall (3.21) together with the definitions

$$
\begin{aligned}
& \mathcal{D}_{\text {apx }}^{\diamond_{0} ;+}(U)=\gamma_{U}^{-3} S^{+}\left[\partial^{(2)} U\right], \\
& \mathcal{D}_{\text {lin; } ;}^{\diamond_{0} ;+}[V]=3 \gamma_{U}^{-5} \partial^{0} U S^{+}\left[\partial^{(2)} U\right] \partial^{0} V+\gamma_{U}^{-3} S^{+}\left[\partial^{(2)} V\right]
\end{aligned}
$$

and write

$$
\mathcal{D}_{\mathrm{nl} ; U}^{\diamond_{0} ;+}(V)=\mathcal{D}^{\diamond_{0} ;+}(U+V)-\mathcal{D}^{\diamond_{0} ;+}(U)-\mathcal{D}_{\operatorname{lin} ; U}^{\diamond_{0} ;+}[V] .
$$

Lemma F.5 Fix $0<\kappa<\frac{1}{12}$. Then there exists $K>0$ so that the pointwise approximation estimate

$$
\left|\mathcal{D}^{\diamond_{0} ;+}(U)-\mathcal{D}_{\mathrm{apx}}^{\diamond_{0} ;+}(U)\right| \leq K h\left[\left|\partial^{(2)} U\right|+T^{+}\left|\partial^{(2)} U\right|\right]
$$

and the residual bound

$$
\begin{aligned}
\left|\mathcal{D}_{\mathrm{nl} ; U}^{\diamond_{0} ;}(V)\right| \leq & K\left[\left|\partial^{-} V\right|^{2}+\left|\partial^{+} V\right|^{2}+T^{+}\left|\partial^{+} V\right|^{2}\right] \\
& +K\left[\left|\partial^{-} V\right|+\left|\partial^{+} V\right|+T^{+}\left|\partial^{+} V\right|\right]\left[\left|\partial^{(2)} V\right|+T^{+}\left|\partial^{(2)} V\right|\right] \\
& +K h\left[\left|\partial^{-} V\right|+\left|\partial^{+} V\right|+T^{+}\left|\partial^{+} V\right|+\left|\partial^{(2)} V\right|+T^{+}\left|\partial^{(2)} V\right|\right]
\end{aligned}
$$

both hold for any $h>0$, any $U \in \Omega_{h ; \kappa}$ and any $V \in \ell_{h}^{2}$ for which $U+V \in \Omega_{h ; \kappa}$.

Proof Motivated by the identity

$$
\begin{aligned}
\mathcal{D}^{\diamond^{0} ;+}(U)= & \frac{1}{2 T^{+} r_{U}^{0}}\left[1+\mathcal{D}^{\diamond_{0}}(U) \mathcal{D}^{\diamond_{0}}(U)\right]\left[\partial^{(2)} U\right] \\
& +\frac{1}{2 T^{+} r_{U}^{0}}\left[1+\mathcal{D}^{\diamond_{0}}(U) T^{+}\left[\mathcal{D}^{\diamond_{0}}(U)\right]\right] T^{+}\left[\partial^{(2)} U\right]
\end{aligned}
$$

derived in Lemma C.3, we write

$$
\begin{aligned}
& f_{1}\left(y_{s}, z, z_{s}\right)=\frac{1}{2 y_{s}}\left[1+z^{2}\right], \\
& f_{2}\left(y_{s}, z, z_{s}\right)=\frac{1}{2 y_{s}}\left[1+z z_{s}\right]
\end{aligned}
$$

together with

$$
\phi\left(\partial^{-} U, \partial^{+} U, T^{+} \partial^{+} U\right)=\left(T^{+} r_{U}^{0}, \mathcal{D}^{\diamond}(U), T^{+} \mathcal{D}^{\diamond}(U)\right)
$$


and finally

$$
\begin{aligned}
P_{1}\left(\partial^{-} U, \partial^{+} U, T^{+} \partial^{+} U, \partial^{(2)} U\right) & =f_{1}\left(\phi\left(\partial^{-} U, \partial^{+} U, T^{+} \partial^{+} U\right)\right) \partial^{(2)} U \\
P_{2}\left(\partial^{-} U, \partial^{+} U, T^{+} \partial^{+} U, T^{+} \partial^{(2)} U\right) & =f_{2}\left(\phi\left(\partial^{-} U, \partial^{+} U, T^{+} \partial^{+} U\right)\right) T^{+} \partial^{(2)} U .(
\end{aligned}
$$

For convenience, we introduce the shorthand

$$
\omega_{U}=\left(\partial^{-} U, \partial^{+} U, T^{+} \partial^{+} U\right) .
$$

This allows one to verify that

$$
\mathcal{D}^{\diamond_{0} ;+}(U)=P_{1}\left(\omega_{U}, \partial^{(2)} U\right)+P_{2}\left(\omega_{U}, T^{+} \partial^{(2)} U\right) .
$$

We now define the approximants

$$
\begin{aligned}
\phi_{\text {apx }}\left(\omega_{U}\right) & =\left(\gamma_{U}, \gamma_{U}^{-1} \partial^{0} U, \gamma_{U}^{-1} \partial^{0} U\right), \\
\phi_{\text {lin } ; U}\left[\omega_{V}\right] & =\left(-\gamma_{U}^{-1}\left[\partial^{0} U\right] \partial^{0} V, \gamma_{U}^{-3} \partial^{0} V, \gamma_{U}^{-3} \partial^{0} V\right) .
\end{aligned}
$$

This allows us to compute

$$
\begin{aligned}
\phi\left(\omega_{U}\right)-\phi_{\mathrm{apx}}\left(\omega_{U}\right)= & \left(T^{+} r_{U}^{0}-\gamma_{U}, \mathcal{D}^{\diamond_{0}}(U)-\mathcal{D}_{\mathrm{apx}}^{\diamond}(U), T^{+} \mathcal{D}^{\diamond_{0}}(U)-\mathcal{D}_{\mathrm{apx}}^{\diamond}(U)\right) \\
= & \left(r_{U}^{0}-\gamma_{U}, \mathcal{D}^{\diamond_{0}}(U)-\mathcal{D}_{\mathrm{apx}}^{\diamond_{0}}(U), \mathcal{D}^{\diamond_{0}}(U)-\mathcal{D}_{\mathrm{apx}}^{\diamond_{0}}(U)\right) \\
& +\left(h \partial^{+}\left[r_{U}^{0}\right], 0, h \mathcal{D}^{\diamond_{0} ;+}(U)\right),
\end{aligned}
$$

together with

$$
\begin{aligned}
\phi_{\mathrm{nl} ; U}\left(\omega_{V}\right)= & \left(T^{+} r_{\mathrm{nl} ; U}^{0}(V), \mathcal{D}_{\mathrm{nl} ; U}^{\diamond_{0}}(V), T^{+} \mathcal{D}_{\mathrm{nl} ; U}^{\triangleright_{0}}(V)\right) \\
& +h\left(-\partial^{+}\left[\gamma_{U}^{-1} \partial^{0} U \partial^{0} V\right], 0, \partial^{+}\left[\gamma_{U}^{-3} \partial^{0} V\right]\right) .
\end{aligned}
$$

In particular, Lemma's F.1 and F.2 together with Corollaries D.3 and D.6 provide the bound

$$
\left|\phi\left(\omega_{U}\right)-\phi_{\mathrm{apx}}\left(\omega_{U}\right)\right| \leq C_{1}^{\prime} h\left[\left|\partial^{(2)} U\right|+T^{+}\left|\partial^{(2)} U\right|\right]
$$

together with

$$
\begin{aligned}
\left|\phi_{\mathrm{nl} ; U}\left(\omega_{V}\right)\right| \leq & C_{1}^{\prime}\left[\left|\partial^{-} V\right|^{2}+\left|\partial^{+} V\right|^{2}+T^{+}\left|\partial^{+} V\right|^{2}\right] \\
& +C_{1}^{\prime} h\left[\left|\partial^{-} V\right|+\left|\partial^{+} V\right|+T^{+}\left|\partial^{+} V\right|+\left|\partial^{(2)} V\right|+T^{+}\left|\partial^{(2)} V\right|\right] .
\end{aligned}
$$

Introducing the compressed nonlinearity

$$
\bar{f}(y, z)=f_{1}(y, z, z)=f_{2}(y, z, z)=\frac{1}{2 y}\left(1+z^{2}\right),
$$

together with the compressed approximants

$$
\begin{aligned}
\bar{\phi}_{\mathrm{apx}}\left(\omega_{U}\right) & =\left(\gamma_{U}, \gamma_{U}^{-1} \partial^{0} U\right), \\
\bar{\phi}_{\operatorname{lin} ; U}\left[\omega_{V}\right] & =\left(-\gamma_{U}^{-1} \partial^{0} U \partial^{0} V, \gamma_{U}^{-3} \partial^{0} V\right),
\end{aligned}
$$

we see that the identities

$$
\begin{aligned}
f_{i}\left(\phi_{\mathrm{apx}}\left(\omega_{U}\right)\right) & =\bar{f}\left(\bar{\phi}_{\mathrm{apx}}\left(\omega_{U}\right)\right), \\
D f_{i}\left(\phi_{\mathrm{apx}}\left(\omega_{U}\right)\right) \phi_{\mathrm{lin} ; U}\left[\omega_{V}\right] & =D \bar{f}\left(\bar{\phi}_{\mathrm{apx}}\left(\omega_{U}\right)\right) \bar{\phi}_{\operatorname{lin} ; U}\left[\omega_{V}\right]
\end{aligned}
$$


hold for $i=1,2$. Upon computing

$$
D \bar{f}(y, z)=\left(-\frac{1}{2 y^{2}}\left(1+z^{2}\right), \frac{z}{y}\right),
$$

we hence see that the functions defined in (E.18) satisfy

$$
\begin{aligned}
P_{1 ; \text { apx }}\left(\omega_{U}, \partial^{(2)} U\right) & =\frac{1}{2} \gamma_{U}^{-1}\left(1+\left(\partial^{0} U\right)^{2} \gamma_{U}^{-2}\right) \partial^{(2)} U \\
& =\frac{1}{2} \gamma_{U}^{-3} \partial^{(2)} U, \\
P_{2 ; \text { apx }}\left(\omega_{U}, T^{+} \partial^{(2)} U\right) & =\frac{1}{2} \gamma_{U}^{-3} T^{+} \partial^{(2)} U,
\end{aligned}
$$

together with

$$
\begin{aligned}
P_{1 ; \operatorname{lin} ; U}\left[\omega_{V}, \partial^{(2)} V\right]= & -\frac{1}{2} \gamma_{U}^{-2}\left(1+\left(\partial^{0} U\right)^{2} \gamma_{U}^{-2}\right)\left(-\gamma_{U}^{-1} \partial^{0} U\right) \partial^{0} V\left(\partial^{(2)} U\right) \\
& +\gamma_{U}^{-2} \partial^{0} U\left(\gamma_{U}^{-3} \partial^{0} V\right)\left(\partial^{(2)} U\right) \\
& +\frac{1}{2} \gamma_{U}^{-1}\left(1+\left(\partial^{0} U\right)^{2} \gamma_{U}^{-2}\right) \partial^{(2)} V \\
= & \frac{3}{2} \gamma_{U}^{-5} \partial^{0} U\left[\partial^{(2)} U\right] \partial^{0} V+\frac{1}{2} \gamma_{U}^{-3} \partial^{(2)} V, \\
P_{2 ; \operatorname{lin} ; U}\left[\omega_{V}, T^{+} \partial^{(2)} V\right]= & \frac{3}{2} \gamma_{U}^{-5} \partial^{0} U T^{+}\left[\partial^{(2)} U\right] \partial^{0} V+\frac{1}{2} \gamma_{U}^{-3} T^{+}\left[\partial^{(2)} V\right] .
\end{aligned}
$$

The desired estimates again follow directly from Corollary E.2.

Proof of Proposition 7.3 The results follow directly from Lemma's F.3, F.4 and F.5 .

\section{F.4 Third Order Discrete Derivatives}

We recall (3.28) together with the definitions

$$
\mathcal{I}_{+; \text {apx }}^{\diamond \diamond_{0} ;+}(U)=\gamma_{U}^{-4}, \quad \mathcal{I}_{+; \text {lin; } U}^{\diamond_{0} ;+}[V]=4 \gamma_{U}^{-6} \partial^{0} U \partial^{0} V
$$

and write

$$
\mathcal{I}_{+; \text {nl } ; U}^{\diamond_{0} ;+}(V)=\mathcal{I}_{+}^{\diamond_{0} ;+}(U+V)-\mathcal{I}_{+}^{\diamond_{0} ;+}(U)-\mathcal{I}_{+; \operatorname{lin} ; U}^{\diamond_{0} ;+}[V]
$$

Lemma F.6 Fix $0<\kappa<\frac{1}{12}$. Then there exists $K>0$ so that the pointwise approximation estimate

$$
\left|\mathcal{I}_{+}^{\diamond_{0} ;+}(U)-\mathcal{I}_{+; \mathrm{apx}}^{\diamond_{0} ;+}(U)\right| \leq K h\left|\partial^{(2)} U\right|
$$

and the residual bound

$$
\left|\mathcal{I}_{+; \mathrm{nl} ; U}^{\diamond \otimes_{0} ;+}(V)\right| \leq K\left[\left|\partial^{-} V\right|^{2}+\left|\partial^{+} V\right|^{2}\right]+K h\left[\left|\partial^{-} V\right|+\left|\partial^{+} V\right|+\left|\partial^{(2)} V\right|\right]
$$

both hold for any $h>0$, any $U \in \Omega_{h ; \kappa}$ and any $V \in \ell_{h}^{2}$ for which $U+V \in \Omega_{h ; \kappa}$.

Proof Motivated by the identity

$$
\mathcal{I}_{+}^{\diamond_{0} ;+}(U)=\frac{1}{r_{U}^{+} r_{U}^{0}}\left[1+\mathcal{D}^{\diamond_{-}}(U) \mathcal{D}^{\diamond_{0}}(U)\right]
$$


derived in Lemma C.4, we may reuse the functions $f, \phi, \phi_{\mathrm{apx}}$ and $\phi_{\text {lin }}$ defined in the proof of Lemma F.4. Writing

$$
P\left(\partial^{-} U, \partial^{+} U\right)=f\left(\phi\left(\partial^{-} U, \partial^{+} U\right)\right),
$$

we conclude that

$$
\mathcal{I}_{+}^{\diamond \diamond_{0} ;+}(U)=P\left(\partial^{-} U, \partial^{+} U\right) .
$$

Reusing the computations in the proof of Lemma F.4, we see that the functions defined in (E.7) satisfy

$$
P_{\text {apx }}(U)=\gamma_{U}^{-2}\left(1+\left(\partial^{0} U\right)^{2} \gamma_{U}^{-2}\right)=\gamma_{U}^{-4},
$$

together with

$$
\begin{aligned}
P_{\text {lin } ; U}[V] & =-2 \gamma_{U}^{-3}\left(1+\left(\partial^{0} U\right)^{2} \gamma_{U}^{-2}\right)\left(-\gamma_{U}^{-1} \partial^{0} U\right) \partial^{0} V+2 \gamma_{U}^{-3} \partial^{0} U\left(\gamma_{U}^{-3} \partial^{0} V\right) \\
& =4 \gamma_{U}^{-6}\left[\partial^{0} U\right] \partial^{0} V .
\end{aligned}
$$

The desired estimates now follow from Lemma E.1 and the bounds (F.34)-(F.35).

We again recall (3.28), but now together with the definitions

$$
\begin{aligned}
& \mathcal{I}_{0 s ; \text { apx }}^{\diamond_{0} ;+}(U)=\mathcal{I}_{s s ; \text { apx }}^{\diamond_{0} ;+}(U)=2 \gamma_{U}^{-6} \partial^{0} U, \\
& \mathcal{I}_{0 s ; \operatorname{lin} ; U}^{\diamond_{0} ;+}[V]=\mathcal{I}_{s s ; \operatorname{lin} ; U}^{\diamond_{0} ;+}[V]=2\left[6 \gamma_{U}^{-8}-5 \gamma_{U}^{-6}\right] \partial^{0} V
\end{aligned}
$$

and write

$$
\begin{aligned}
& \mathcal{I}_{0 s ; \mathrm{nl} ; U}^{\diamond \diamond_{0} ;+}(V)=\mathcal{I}_{0 s}^{\diamond \diamond_{0} ;+}(U+V)-\mathcal{I}_{0 s}^{\diamond \diamond_{0} ;+}(U)-\mathcal{I}_{0 s ; \operatorname{lin} ; U}^{\diamond_{0} ;+}[V], \\
& \mathcal{I}_{s s ; n ; ~}^{\diamond \diamond_{0} ;+}(V)=\mathcal{I}_{s s}^{\diamond \diamond_{0} ;+}(U+V)-\mathcal{I}_{s s}^{\diamond \diamond_{0} ;+}(U)-\mathcal{I}_{s s ; \operatorname{lin} ; U}^{\diamond_{0} ;+}[V] .
\end{aligned}
$$

Lemma F.7 Fix $0<\kappa<\frac{1}{12}$. Then there exists $K>0$ so that the pointwise approximation estimate

$$
\left|\mathcal{I}_{0 s}^{\diamond_{0} ;+}(U)-\mathcal{I}_{s ; \text { apx }}^{\diamond_{0} ;+}(U)\right|+\left|\mathcal{I}_{s s}^{\diamond_{0} ;+}(U)-\mathcal{I}_{s ; \mathrm{apx}}^{\diamond_{0} ;+}(U)\right| \leq K h\left[\left|\partial^{(2)} U\right|+T^{+}\left|\partial^{(2)} U\right|\right]
$$

and the residual bound

$$
\begin{aligned}
\left|\mathcal{I}_{0 s ; \mathrm{nl} ; U}^{\diamond_{0} ;+}(V)\right|+\left|\mathcal{I}_{s s ; \mathrm{nl} ; U}^{\otimes_{0} ;+}(V)\right| \leq & K\left[\left|\partial^{-} V\right|^{2}+\left|\partial^{+} V\right|^{2}+T^{+}\left|\partial^{+} V\right|^{2}\right] \\
& +K h\left[\left|\partial^{-} V\right|+\left|\partial^{+} V\right|+T^{+}\left|\partial^{+} V\right|+\left|\partial^{(2)} V\right|+T^{+}\left|\partial^{(2)} V\right|\right]
\end{aligned}
$$

both hold for any $h>0$, any $U \in \Omega_{h ; \kappa}$ and any $V \in \ell_{h}^{2}$ for which $U+V \in \Omega_{h ; \kappa}$.

Proof For convenience, we introduce the shorthand

$$
\omega_{U}=\left(\partial^{-} U, \partial^{+} U, T^{+} \partial^{+} U\right) .
$$

Motivated by the identities derived in Lemma C.4, we write

$$
\begin{aligned}
f_{0 s}\left(y_{0}, y_{0 s}, y_{+}, y_{+s}, z_{0}, z_{0 s}, z_{-}, z_{+}\right)= & \frac{1}{2 y_{+} y_{0} y_{0 s}} z_{0}\left(1+z_{+} z_{0 s}\right) \\
& +\frac{1}{y_{0} y_{+} y_{+}} z_{0 s}\left(1+z_{-} z_{0}\right)+\frac{1}{2 y_{+} y_{0} y_{0 s}} z_{-}\left(1+z_{0}^{2}\right), \\
f_{s s}\left(y_{0}, y_{0 s}, y_{+}, y_{+s}, z_{0}, z_{0 s}, z_{-}, z_{+}\right)= & \frac{2 y_{0}+y_{+s}}{2 y_{0} y_{0 s} y_{+} y_{+s}} z_{0 s}\left(1+z_{+} z_{0 s}\right) \\
& +\frac{1}{2 y_{+} y_{0} y_{0 s}} z_{-}\left(1+z_{0} z_{0 s}\right),
\end{aligned}
$$


together with

$$
\phi\left(\omega_{U}\right)=\left(r_{U}^{0}, T^{+} r_{U}^{0}, r_{U}^{+}, T^{+} r_{U}^{+}, \mathcal{D}^{\diamond}(U), T^{+} \mathcal{D}^{\diamond}(U), \mathcal{D}^{\diamond}(U), \mathcal{D}^{\diamond+}(U)\right)
$$

and finally

$$
P_{0 s}\left(\omega_{U}\right)=f_{0 s}\left(\phi\left(\omega_{U}\right)\right), \quad P_{s s}\left(\omega_{U}\right)=f_{s s}\left(\phi\left(\omega_{U}\right)\right) .
$$

This allows one to verify that

$$
\mathcal{I}_{0 s}^{\diamond \diamond_{0} ;+}(U)=P_{0 s}\left(\omega_{U}\right), \quad \mathcal{I}_{s s}^{\diamond \diamond_{0} ;+}(U)=P_{s s}\left(\omega_{U}\right) .
$$

We now define the approximants

$$
\begin{aligned}
\phi_{\mathrm{apx}}\left(\omega_{U}\right)= & \left(\gamma_{U}, \gamma_{U}, \gamma_{U}, \gamma_{U}, \gamma_{U}^{-1} \partial^{0} U, \gamma_{U}^{-1} \partial^{0} U, \gamma_{U}^{-1} \partial^{0} U, \gamma_{U}^{-1} \partial^{0} U\right), \\
\phi_{\operatorname{lin} ; U}\left[\omega_{V}\right]= & \left(-\gamma_{U}^{-1} \partial^{0} U \partial^{0} V,-\gamma_{U}^{-1} \partial^{0} U \partial^{0} V,-\gamma_{U}^{-1} \partial^{0} U \partial^{0} V,-\gamma_{U}^{-1} \partial^{0} U \partial^{0} V,\right. \\
& \left.\gamma_{U}^{-3} \partial^{0} V, \gamma_{U}^{-3} \partial^{0} V, \gamma_{U}^{-3} \partial^{0} V, \gamma_{U}^{-3} \partial^{0} V\right) .
\end{aligned}
$$

This allows us to compute

$$
\begin{aligned}
\phi\left(\omega_{U}\right)-\phi_{\mathrm{apx}}\left(\omega_{U}\right)= & \left(r_{U}^{0}-\gamma_{U}, r_{U}^{0}-\gamma_{U}, r_{U}^{+}-\gamma_{U}, r_{U}^{+}-\gamma_{U},\right. \\
& \mathcal{D}^{\diamond_{0}}(U)-\mathcal{D}_{\mathrm{apx}}^{\diamond_{0}}(U), \mathcal{D}^{\diamond_{0}}(U)-\mathcal{D}_{\mathrm{apx}}^{\diamond_{0}}(U), \\
& \left.\mathcal{D}^{\diamond_{-}}(U)-\mathcal{D}_{\mathrm{apx}}^{\diamond_{-}}(U), \mathcal{D}^{\diamond_{+}}(U)-\mathcal{D}_{\mathrm{apx}}^{\diamond_{+}}(U)\right) \\
& +h\left(0, \partial^{+}\left[r_{U}^{0}\right], 0, \partial^{+}\left[r_{U}^{+}\right], 0, \mathcal{D}^{\diamond_{0} ;+}(U), 0,0\right),
\end{aligned}
$$

together with

$$
\begin{aligned}
\phi_{\mathrm{nl} ; U}\left(\omega_{V}\right)= & \left(r_{\mathrm{nl} ; U}^{0}(V), T^{+} r_{\mathrm{nl} ; U}^{0}(V), r_{\mathrm{nl} ; U}^{+}(V), T^{+} r_{\mathrm{nl} ; U}^{+}(V),\right. \\
& \left.\mathcal{D}_{\mathrm{nl} ; U}^{\diamond_{0}}(V), T^{+} \mathcal{D}_{\mathrm{nl} ; U}^{\diamond_{0}}(V), \mathcal{D}_{\mathrm{nl} ; U}^{\diamond_{-}}(V), \mathcal{D}_{\mathrm{nl} ; U}^{\diamond_{+}}(V)\right) \\
& +h\left(0,-\partial^{+}\left[\gamma_{U}^{-1} \partial^{0} U \partial^{0} V\right], 0,-\partial^{+}\left[\gamma_{U}^{-1} \partial^{0} U \partial^{0} V\right],\right. \\
& \left.0, \partial^{+}\left[\gamma_{U}^{-3} \partial^{0} V\right], 0,0\right) .
\end{aligned}
$$

In particular, Lemma's F.1 and F.2 together with Corollaries D.3 and D.6 provide the bound

$$
\left|\phi\left(\omega_{U}\right)-\phi_{\text {apx }}\left(\omega_{U}\right)\right| \leq C_{1}^{\prime} h\left[\left|\partial^{(2)} U\right|+T^{+}\left|\partial^{(2)} U\right|\right]
$$

together with

$$
\begin{aligned}
\left|\phi_{\mathrm{nl} ; U}\left(\omega_{V}\right)\right| \leq & C_{1}^{\prime}\left[\left|\partial^{-} V\right|^{2}+\left|\partial^{+} V\right|^{2}+T^{+}\left|\partial^{+} V\right|^{2}\right] \\
& +C_{1}^{\prime} h\left[\left|\partial^{-} V\right|+\left|\partial^{+} V\right|+T^{+}\left|\partial^{+} V\right|+\left|\partial^{(2)} V\right|+T^{+}\left|\partial^{(2)} V\right|\right] .
\end{aligned}
$$

Introducing the compressed nonlinearity

$$
\bar{f}(y, z)=f_{0 s}(y, y, y, y, z, z, z, z)=f_{s s}(y, y, y, y, z, z, z, z)=\frac{2 z}{y^{3}}+\frac{2 z^{3}}{y^{3}},
$$

together with the compressed approximants

$$
\bar{\phi}_{\mathrm{apx}}\left(\omega_{U}\right)=\left(\gamma_{U}, \gamma_{U}^{-1} \partial^{0} U\right),
$$




$$
\bar{\phi}_{\operatorname{lin} ; U}\left[\omega_{V}\right]=\left(-\gamma_{U}^{-1} \partial^{0} U \partial^{0} V, \gamma_{U}^{-3} \partial^{0} V\right),
$$

we see that the identities

$$
\begin{aligned}
f_{\#}\left(\phi_{\mathrm{apx}}\left(\omega_{U}\right)\right) & =\bar{f}\left(\bar{\phi}_{\mathrm{apx}}\left(\omega_{U}\right)\right), \\
D f_{\#}\left(\phi_{\mathrm{apx}}\left(\omega_{U}\right)\right) \phi_{\operatorname{lin} ; U}\left[\omega_{V}\right] & =D \bar{f}\left(\bar{\phi}_{\mathrm{apx}}\left(\omega_{U}\right)\right) \bar{\phi}_{\operatorname{lin} ; U}\left[\omega_{V}\right]
\end{aligned}
$$

hold for \# $\in\{0 s, s s\}$. Upon computing

$$
D \bar{f}(y, z)=\left(-6 \frac{z+z^{3}}{y^{4}}, \frac{2+6 z^{2}}{y^{3}}\right),
$$

we hence see that the functions defined in (E.7) satisfy

$$
\begin{aligned}
P_{0 s ; \text { apx }}\left(\omega_{U}\right) & =P_{s s ; \text { apx }}\left(\omega_{U}\right) \\
& =2 \gamma_{U}^{-4} \partial^{0} U+2 \gamma_{U}^{-6}\left(\partial^{0} U\right)^{3} \\
& =2 \gamma_{U}^{-6} \partial^{0} U,
\end{aligned}
$$

together with

$$
\begin{aligned}
P_{0 s ; \operatorname{lin} ; U}\left[\omega_{V}\right]= & P_{s s ; \operatorname{lin} ; U\left[\omega_{V}\right]} \\
= & -6 \gamma_{U}^{-5} \partial^{0} U\left(1+\gamma_{U}^{-2}\left(\partial^{0} U\right)^{2}\right)\left(-\gamma_{U}^{-1} \partial^{0} U \partial^{0} V\right) \\
& +\left(2 \gamma_{U}^{-3}+6 \gamma_{U}^{-5}\left(\partial^{0} U\right)^{2}\right) \gamma_{U}^{-3} \partial^{0} V \\
= & {\left[6 \gamma_{U}^{-8}\left(1-\gamma_{U}^{2}\right)+2 \gamma_{U}^{-6}+6 \gamma_{U}^{-8}\left(1-\gamma_{U}^{2}\right)\right] \partial^{0} V } \\
= & {\left[-10 \gamma_{U}^{-6}+12 \gamma_{U}^{-8}\right] \partial^{0} V . }
\end{aligned}
$$

The desired estimates now follow from Lemma E.1.

\section{F.5 Auxiliary Functions}

We recall (4.2) together with the definitions

$$
\begin{aligned}
\tilde{p}_{\text {apx }}(U) & =\gamma_{U}^{2}, \\
\widetilde{p}_{\text {lin } ; U}[V] & =-2 \partial^{0} U \partial^{0} V, \\
p_{\text {apx }}(U) & =\gamma_{U} \partial^{0} U, \\
p_{\text {lin } ; U}[V] & =\gamma_{U}^{-1}\left(2 \gamma_{U}^{2}-1\right) \partial^{0} V
\end{aligned}
$$

and write

$$
\begin{aligned}
& \widetilde{p}_{\mathrm{nl} ; U}(V)=\widetilde{p}(U+V)-\widetilde{p}(U)-\widetilde{p}_{\mathrm{lin} ; U}[V], \\
& p_{\mathrm{nl} ; U}(V)=p(U+V)-p(U)-p_{\mathrm{lin} ; U}[V] .
\end{aligned}
$$

Lemma F.8 Fix $0<\kappa<\frac{1}{12}$. Then there exists $K>0$ so that the pointwise approximation estimate

$$
\left|\widetilde{p}(U)-\widetilde{p}_{\text {apx }}(U)\right|+\left|p(U)-p_{\text {apx }}(U)\right| \leq K h\left|\partial^{(2)} U\right|
$$

and the residual bound

$$
\left|\tilde{p}_{\mathrm{nl} ; U}(V)\right|+\left|p_{\mathrm{nl} ; U}(V)\right| \leq K\left[\left|\partial^{-} V\right|^{2}+\left|\partial^{+} V\right|^{2}\right]
$$




$$
+K h\left[\left|\partial^{-} V\right|+\left|\partial^{+} V\right|+\left|\partial^{(2)} V\right|\right]
$$

both hold for any $h>0$, any $U \in \Omega_{h ; \kappa}$ and any $V \in \ell_{h}^{2}$ for which $U+V \in \Omega_{h ; \kappa}$.

Proof Motivated by the definitions (4.2), we write

$$
f_{\widetilde{p}}\left(z_{0}, z_{+}\right)=\frac{1}{1+z_{+} z_{0}}, \quad f_{p}\left(z_{0}, z_{+}\right)=\frac{z_{+}}{1+z_{+} z_{0}},
$$

together with

$$
\phi\left(\partial^{-} U, \partial^{+} U\right)=\left(\mathcal{D}^{\diamond}(U), \mathcal{D}^{\diamond}(U)\right)
$$

and finally

$$
\begin{aligned}
& \widetilde{P}\left(\partial^{-} U, \partial^{+} U\right)=f_{\widetilde{p}}\left(\phi\left(\partial^{-} U, \partial^{+} U\right)\right), \\
& P\left(\partial^{-} U, \partial^{+} U\right)=f_{p}\left(\phi\left(\partial^{-} U, \partial^{+} U\right)\right) .
\end{aligned}
$$

This allows one to verify that

$$
\widetilde{p}(U)=\widetilde{P}\left(\partial^{-} U, \partial^{+} U\right), \quad p(U)=P\left(\partial^{-} U, \partial^{+} U\right) .
$$

We now define the approximants

$$
\begin{aligned}
& \phi_{\text {apx }}\left(\partial^{-} U, \partial^{+} U\right)=\left(\gamma_{U}^{-1} \partial^{0} U, \gamma_{U}^{-1} \partial^{0} U\right), \\
& \phi_{\operatorname{lin} ; U}\left[\partial^{-} V, \partial^{+} V\right]=\left(\gamma_{U}^{-3} \partial^{0} V, \gamma_{U}^{-3} \partial^{0} V\right) .
\end{aligned}
$$

This allows us to compute

$$
\phi\left(\partial^{-} U, \partial^{+} U\right)-\phi_{\text {apx }}\left(\partial^{-} U, \partial^{+} U\right)=\left(\mathcal{D}^{\diamond_{0}}(U)-\mathcal{D}_{\text {apx }}^{\diamond_{0}}(U), \mathcal{D}^{\diamond_{+}}(U)-\mathcal{D}_{\text {apx }}^{\diamond_{+}}(U)\right),
$$

together with

$$
\phi_{\mathrm{nl} ; U}\left(\partial^{-} V, \partial^{+} V\right)=\left(\mathcal{D}_{\mathrm{nl} ; U}^{\diamond_{0}}(V), \mathcal{D}_{\mathrm{nl} ; U}^{\diamond_{+}}(V)\right) .
$$

In particular, Lemma F.2 provides the bound

$$
\left|\phi\left(\partial^{-} U, \partial^{+} U\right)-\phi_{\text {apx }}\left(\partial^{-} U, \partial^{+} U\right)\right| \leq K h\left|\partial^{(2)} U\right|
$$

together with

$$
\begin{aligned}
\left|\phi_{\mathrm{nl} ; U}\left(\partial^{-} V, \partial^{+} V\right)\right| \leq & K\left[\left|\partial^{-} V\right|^{2}+\left|\partial^{+} V\right|^{2}\right] \\
& +K h\left[\left|\partial^{-} V\right|+\left|\partial^{+} V\right|+\left|\partial^{(2)} V\right|\right] .
\end{aligned}
$$

Introducing the compressed nonlinearities

$$
\begin{aligned}
& \bar{f}_{\tilde{p}}(z)=f_{\widetilde{p}}(z, z)=\frac{1}{1+z^{2}}, \\
& \bar{f}_{p}(z)=f_{p}(z, z)=\frac{z}{1+z^{2}},
\end{aligned}
$$

together with the compressed approximants

$$
\bar{\phi}_{\text {apx }}\left(\partial^{-} U, \partial^{+} U\right)=\gamma_{U}^{-1} \partial^{0} U, \quad \bar{\phi}_{\text {lin; } U}\left[\partial^{-} V, \partial^{+} V\right]=\gamma_{U}^{-3} \partial^{0} V,
$$


we see that the identities

$$
\begin{aligned}
f_{\#}\left(\phi_{\text {apx }}\left(\partial^{-} U, \partial^{+} U\right)\right) & =\bar{f}_{\#}\left(\bar{\phi}_{\text {apx }}\left(\partial^{-} U, \partial^{+} U\right)\right), \\
D f_{\#}\left(\phi_{\text {apx }}\left(\partial^{-} U, \partial^{+} U\right)\right) \phi_{\operatorname{lin} ; U}\left[\partial^{-} V, \partial^{+} V\right] & =D \bar{f}_{\#}\left(\bar{\phi}_{\text {apx }}\left(\partial^{-} U, \partial^{+} U\right)\right) \bar{\phi}_{\operatorname{lin} ; U}\left[\partial^{-} V, \partial^{+} V\right]
\end{aligned}
$$

hold for $\# \in\{\tilde{p}, p\}$. Upon computing

$$
\bar{f}_{\widetilde{p}}^{\prime}(z)=-\frac{2 z}{\left(1+z^{2}\right)^{2}}, \quad \bar{f}_{p}^{\prime}(z)=\frac{1-z^{2}}{\left(1+z^{2}\right)^{2}},
$$

we hence see that the functions defined in (E.7) satisfy

$$
\begin{aligned}
& \widetilde{P}_{\text {apx }}(U)=\left[1+\gamma_{U}^{-2}\left(\partial^{0} U\right)^{2}\right]^{-1}=\gamma_{U}^{2}, \\
& P_{\text {apx }}(U)=\gamma_{U}^{-1} \partial^{0} U\left[1+\gamma_{U}^{-2}\left(\partial^{0} U\right)^{2}\right]^{-1}=\gamma_{U} \partial^{0} U,
\end{aligned}
$$

together with

$$
\begin{aligned}
\widetilde{P}_{\text {lin } ; U}[V] & =-2 \gamma_{U}^{-1} \partial^{0} U\left[1+\gamma_{U}^{-2}\left(\partial^{0} U\right)^{2}\right]^{-2} \gamma_{U}^{-3} \partial^{0} V \\
& =-2\left[\partial^{0} U\right] \partial^{0} V \\
P_{\operatorname{lin} ; U}[V] & =\left(1-\gamma_{U}^{-2}\left(\partial^{0} U\right)^{2}\right)\left[1+\gamma_{U}^{-2}\left(\partial^{0} U\right)^{2}\right]^{-2} \gamma_{U}^{-3} \partial^{0} V \\
& =\left(2-\gamma_{U}^{-2}\right) \gamma_{U} \partial^{0} V \\
& =\gamma_{U}^{-1}\left(2 \gamma_{U}^{2}-1\right) \partial^{0} V .
\end{aligned}
$$

The desired estimates now follow from Lemma E.1.

We recall (6.3) together with the definitions

$$
\begin{array}{ll}
p_{\mathrm{apx}}^{\diamond_{0}}(U)=\gamma_{U}^{2}\left(\gamma_{U}^{2}-1\right), & p_{\operatorname{lin} ; U}^{\diamond_{0}}[V]=\left(2-4 \gamma_{U}^{2}\right) \partial^{0} U \partial^{0} V, \\
p_{\mathrm{apx}}^{\diamond_{+}}(U)=\gamma_{U}^{4}, & p_{\operatorname{lin} ; U}^{\diamond_{+}}[V]=-4 \gamma_{U}^{2} \partial^{0} U \partial^{0} V
\end{array}
$$

and write

$$
\begin{aligned}
& p_{\mathrm{nl} ; U}^{\diamond_{0}}(V)=p^{\diamond_{0}}(U+V)-p^{\diamond_{0}}(U)-p_{\operatorname{lin} ; U}^{\diamond_{0}}[V], \\
& p_{\mathrm{nl} ; U}^{\diamond_{+}}(V)=p^{\diamond_{+}}(U+V)-p^{\diamond_{+}}(U)-p_{\operatorname{lin} ; U}^{\diamond_{+}}[V] .
\end{aligned}
$$

Lemma F.9 Fix $0<\kappa<\frac{1}{12}$. Then there exists $K>0$ so that the pointwise approximation estimate

$$
\left|p^{\diamond+}(U)-p_{\text {apx }}^{\diamond_{+}}(U)\right|+\left|p^{\diamond_{0}}(U)-p_{\mathrm{apx}}^{\diamond_{0}}(U)\right| \leq K h\left[\left|\partial^{(2)} U\right|+T^{+}\left|\partial^{(2)} U\right|\right]
$$

and the residual bound

$$
\begin{aligned}
\left|p_{\mathrm{nl} ; U}^{\diamond_{+}}(V)\right|+\left|p_{\mathrm{nl} ; U}^{\diamond_{0}}(V)\right| \leq & K\left[\left|\partial^{-} V\right|^{2}+\left|\partial^{+} V\right|^{2}+T^{+}\left|\partial^{+} V\right|^{2}\right] \\
& +K h\left[\left|\partial^{-} V\right|+\left|\partial^{+} V\right|+T^{+}\left|\partial^{+} V\right|+\left|\partial^{(2)} V\right|+T^{+}\left|\partial^{(2)} V\right|\right]
\end{aligned}
$$

both hold for any $h>0$, any $U \in \Omega_{h ; \kappa}$ and any $V \in \ell_{h}^{2}$ for which $U+V \in \Omega_{h ; \kappa}$. 
Proof For convenience, we introduce the shorthand

$$
\omega_{U}=\left(\partial^{-} U, \partial^{+} U, T^{+} \partial^{+} U\right) .
$$

Motivated by the definitions (6.3), we write

$$
\begin{aligned}
& f_{A}^{\diamond_{+}}\left(z_{0}, z_{0 s}, z_{+}, z_{+s}\right)=\frac{2+z_{+} z_{0}+z_{+s} z_{0 s}}{2\left(1+z_{+} z_{0}\right)\left(1+z_{+s} z_{0 s}\right)}, \\
& f_{B}^{\diamond+}\left(z_{0}, z_{0 s}, z_{+}, z_{+s}\right)=-\frac{\left(z_{+}+z_{+s}\right)\left(z_{0}+z_{0 s}\right)}{4\left(1+z_{+} z_{0}\right)\left(1+z_{+s} z_{0 s}\right)}, \\
& f^{\diamond_{0}}\left(z_{0}, z_{0 s}, z_{+}, z_{+s}\right)=-\frac{\left(z_{+}+z_{+s}\right)^{2}}{4\left(1+z_{+} z_{0}\right)\left(1+z_{+s} z_{0 s}\right)},
\end{aligned}
$$

together with

$$
\phi\left(\omega_{U}\right)=\left(\mathcal{D}^{\diamond}(U), T^{+} \mathcal{D}^{\diamond}(U), \mathcal{D}^{\diamond+}(U), T^{+} \mathcal{D}^{\diamond+}(U)\right)
$$

and finally

$$
\begin{aligned}
& P_{A}^{\diamond_{+}}\left(\omega_{U}\right)=f_{A}^{\diamond_{+}}\left(\phi\left(\omega_{U}\right)\right), \\
& P_{B}^{\diamond_{+}}\left(\omega_{U}\right)=f_{B}^{\diamond_{+}}\left(\phi\left(\omega_{U}\right)\right), P^{\diamond_{0}}\left(\omega_{U}\right)=f^{\diamond_{0}}\left(\phi\left(\omega_{U}\right)\right) .
\end{aligned}
$$

This allows one to verify that

$$
\begin{aligned}
& p_{A}^{\diamond^{+}}(U)=P_{A}^{\diamond^{+}}\left(\omega_{U}\right), \\
& p_{B}^{\diamond_{+}}(U)=P_{B}^{\diamond_{+}}\left(\omega_{U}\right), \quad p^{\diamond_{0}}(U)=P^{\diamond_{0}}\left(\omega_{U}\right) .
\end{aligned}
$$

We now define the approximants

$$
\begin{aligned}
\phi_{\text {apx }}\left(\omega_{U}\right) & =\left(\gamma_{U}^{-1} \partial^{0} U, \gamma_{U}^{-1} \partial^{0} U, \gamma_{U}^{-1} \partial^{0} U, \gamma_{U}^{-1} \partial^{0} U\right), \\
\phi_{\operatorname{lin} ; U}\left[\omega_{V}\right] & =\left(\gamma_{U}^{-3} \partial^{0} V, \gamma_{U}^{-3} \partial^{0} V, \gamma_{U}^{-3} \partial^{0} V, \gamma_{U}^{-3} \partial^{0} V\right) .
\end{aligned}
$$

This allows us to compute

$$
\begin{aligned}
\phi\left(\omega_{U}\right)-\phi_{\mathrm{apx}}\left(\omega_{U}\right)= & \left(\mathcal{D}^{\diamond_{0}}(U)-\mathcal{D}_{\mathrm{apx}}^{\diamond_{0}}(U), \mathcal{D}^{\diamond_{0}}(U)-\mathcal{D}_{\mathrm{apx}}^{\diamond_{0}}(U),\right. \\
& \left.\mathcal{D}^{\diamond_{+}}(U)-\mathcal{D}_{\mathrm{apx}}^{\diamond_{+}}(U), \mathcal{D}^{\diamond_{+}}(U)-\mathcal{D}_{\mathrm{apx}}^{\diamond_{+}}(U)\right) \\
& +h\left(0, \mathcal{D}_{0}^{\diamond_{0}++}(U), 0, \mathcal{D}^{\diamond_{+} ;+}(U)\right),
\end{aligned}
$$

together with

$$
\begin{aligned}
\phi_{\mathrm{nl} ; U}\left(\omega_{V}\right)= & \left(\mathcal{D}_{\mathrm{nl} ; U}^{\diamond_{0}}(V), T^{+} \mathcal{D}_{\mathrm{nl} ; U}^{\diamond_{0}}(V), \mathcal{D}_{\mathrm{nl} ; U}^{\diamond_{+}}(V), T^{+} \mathcal{D}_{\mathrm{nl} ; U}^{\diamond_{+}}(V)\right) \\
& +h\left(0, \partial^{+}\left[\gamma_{U}^{-3} \partial^{0} V\right], 0, \partial^{+}\left[\gamma_{U}^{-3} \partial^{0} V\right]\right) .
\end{aligned}
$$

In particular, Lemma F. 2 provides the bound

$$
\left|\phi\left(\omega_{U}\right)-\phi_{\text {apx }}\left(\omega_{U}\right)\right| \leq K h\left[\left|\partial^{(2)} U\right|+T^{+}\left|\partial^{(2)} U\right|\right],
$$

together with

$$
\begin{aligned}
\left|\phi_{\mathrm{nl} ; U}\left(\omega_{V}\right)\right| \leq & K\left[\left|\partial^{-} V\right|^{2}+\left|\partial^{+} V\right|^{2}+T^{+}\left|\partial^{+} V\right|^{2}\right] \\
& +K h\left[\left|\partial^{-} V\right|+\left|\partial^{+} V\right|+T^{+}\left|\partial^{+} V\right|+\left|\partial^{(2)} V\right|+T^{+}\left|\partial^{(2)} V\right|\right] .
\end{aligned}
$$


Introducing the compressed nonlinearities

$$
\begin{aligned}
\bar{f}_{A}^{\diamond^{+}}(z) & =f_{A}^{\diamond_{+}}(z, z, z, z)=\frac{1}{1+z^{2}}, \\
\bar{f}_{B}^{\diamond^{+}}(z) & =f_{B}^{{ }^{+}}(z, z, z, z) \\
& =-\frac{1}{1+z^{2}}+\frac{1}{\left(1+z^{2}\right)^{2}}, \\
\bar{f}^{\diamond_{0}}(z) & =f^{\diamond_{0}}(z, z, z, z) \\
& =\bar{f}_{B}^{\diamond_{+}}(z),
\end{aligned}
$$

together with the compressed approximants

$$
\bar{\phi}_{\text {apx }}\left(\omega_{U}\right)=\gamma_{U}^{-1} \partial^{0} U, \quad \bar{\phi}_{\operatorname{lin} ; U}\left[\omega_{V}\right]=\gamma_{U}^{-3} \partial^{0} V,
$$

we see that the identities

$$
\begin{aligned}
f_{\#}^{\diamond_{+}}\left(\phi_{\mathrm{apx}}\left(\omega_{U}\right)\right) & =\bar{f}_{\#}^{\diamond_{+}}\left(\bar{\phi}_{\mathrm{apx}}\left(\omega_{U}\right)\right), \\
D f_{\#}^{\diamond+}\left(\phi_{\mathrm{apx}}\left(\omega_{U}\right)\right) \phi_{\operatorname{lin} ; U}\left[\omega_{V}\right] & =D \bar{f}_{\#}^{\diamond_{+}}\left(\bar{\phi}_{\mathrm{apx}}\left(\omega_{U}\right)\right) \bar{\phi}_{\operatorname{lin} ; U}\left[\omega_{V}\right]
\end{aligned}
$$

hold for $\# \in\{A, B\}$, together with similar identities for $f^{\diamond_{0}}$. Upon computing

$$
\begin{aligned}
D \bar{f}_{A}^{\diamond^{+}}(z) & =\frac{-2 z}{\left(1+z^{2}\right)^{2}}, \\
D \bar{f}_{B}^{\diamond^{+}}(z) & =\frac{2 z}{\left(1+z^{2}\right)^{2}}-\frac{4 z}{\left(1+z^{2}\right)^{3}},
\end{aligned}
$$

we hence see that the functions defined in (E.7) satisfy

$$
\begin{aligned}
P_{A ; \text { apx }}^{\diamond_{+}}(U) & =\left[1+\gamma_{U}^{-2}\left(\partial^{0} U\right)^{2}\right]^{-1} \\
& =\gamma_{U}^{2}, \\
P_{B ; \text { apx }}^{\diamond_{+}}(U) & =P_{\mathrm{apx}}^{\diamond_{0}}(U) \\
& =-\gamma_{U}^{-2}\left(\partial^{0} U\right)^{2}\left[1+\gamma_{U}^{-2}\left(\partial^{0} U\right)^{2}\right]^{-2} \\
& =-\gamma_{U}^{2}\left(1-\gamma_{U}^{2}\right) \\
& =\gamma_{U}^{4}-\gamma_{U}^{2},
\end{aligned}
$$

together with

$$
\begin{aligned}
P_{A ; \operatorname{lin} ; U}^{\diamond_{+}}[V] & =-2 \gamma_{U}^{-1} \partial^{0} U\left[1+\gamma_{U}^{-2}\left(\partial^{0} U\right)^{2}\right]^{-2} \gamma_{U}^{-3} \partial^{0} V \\
& =-2 \partial^{0} U \partial^{0} V, \\
P_{B ; \operatorname{lin} ; U}^{\diamond_{+}}[V] & =P_{\operatorname{lin} ; U}^{\diamond_{0}}[V] \\
& =\gamma_{U}^{-1} \partial^{0} U\left[2\left[1+\gamma_{U}^{-2}\left(\partial^{0} U\right)^{2}\right]^{-2}-4\left[1+\gamma_{U}^{-2}\left(\partial^{0} U\right)^{2}\right]^{-3}\right] \gamma_{U}^{-3} \partial^{0} V \\
& =\left[2-4 \gamma_{U}^{2}\right] \partial^{0} U \partial^{0} V .
\end{aligned}
$$

The desired estimates now follow from Lemma E.1.

Proof of Proposition 7.2 The results follow directly from Lemma's F.6-F.9. 


\section{F.6 Estimates for $g$}

We recall the notation $g^{+}(U)=\partial^{+} g(U)$ together with the definitions

$$
\begin{array}{ll}
g_{\text {apx }}(U)=g(U), & g_{\operatorname{lin} ; U}[V]=g^{\prime}(U) V, \\
g_{\text {apx }}^{+}(U)=g^{\prime}(U) \partial^{0} U, & g_{\operatorname{lin} ; U}^{+}[V]=g^{\prime \prime}(U)\left[\partial^{0} U\right] V+g^{\prime}(U) \partial^{0} V
\end{array}
$$

and write

$$
\begin{aligned}
& g_{\mathrm{nl} ; U}(V)=g(U+V)-g(U)-g_{\operatorname{lin} ; U}[V], \\
& g_{\mathrm{nl} ; U}^{+}(V)=g^{+}(U+V)-g^{+}(U)-g_{\operatorname{lin} ; U}^{+}[V] .
\end{aligned}
$$

Lemma F.10 Suppose that $(\mathrm{Hg})$ is satisfied and fix $0<\kappa<\frac{1}{12}$. Then there exists $K>0$ so that the pointwise approximation estimate

$$
\left|g^{+}(U)-g_{\text {apx }}^{+}(U)\right| \leq K h\left[\left|\partial^{(2)} U\right|+\left|\partial^{+} U\right|\right]
$$

and the residual bounds

$$
\begin{aligned}
\left|g_{\mathrm{nl} ; U}(V)\right| \leq & K|V|^{2} \\
\left|g_{\mathrm{nl} ; U}^{+}(V)\right| \leq & K\left[|V|^{2}+T^{+}|V|^{2}+\left|\partial^{+} V\right|^{2}\right] \\
& +K h\left[|V|+T^{+}|V|+\left|\partial^{+} V\right|+\left|\partial^{(2)} V\right|\right]
\end{aligned}
$$

all hold for any $h>0$, any $U \in \Omega_{h ; \kappa}$ and any $V \in \ell_{h}^{2}$ for which $U+V \in \Omega_{h ; \kappa}$.

Proof We first note that

$$
\begin{aligned}
g_{\mathrm{nl} ; U}(V) & =g(U+V)-g(U)-g^{\prime}(U) V \\
& =\int_{0}^{1} \int_{0}^{\sigma} g^{\prime \prime}\left(U+\sigma^{\prime} V\right) V^{2} d \sigma^{\prime} d \sigma,
\end{aligned}
$$

which yields the desired estimate for $g_{\mathrm{nl}}$. In addition, for any pair $\left(U^{(1)}, U^{(2)}\right) \in \Omega_{h ; \kappa}^{2}$, the $C^{3}$-smoothness of $g$ implies the pointwise bound

$$
\left|g_{\mathrm{nl} ; U^{(1)}}(V)-g_{\mathrm{nl} ; U^{(2)}}(V)\right| \leq C_{1}^{\prime}\left|U^{(1)}-U^{(2)}\right| V^{2} .
$$

Finally, upon writing

$$
g_{\mathrm{nl} ; U}^{(1)}(V)=g^{\prime}(U+V)-g^{\prime}(U)-g^{\prime \prime}(U) V,
$$

the $C^{3}$-smoothness of $g$ implies the bound

$$
\left|g_{\mathrm{nl} ; U}^{(1)}(V)\right| \leq C_{2}^{\prime}|V|^{2} .
$$

We now compute

$$
\begin{aligned}
g^{+}(U) & =h^{-1}\left[g\left(T^{+} U\right)-g(U)\right] \\
& =g^{\prime}(U) \partial^{+} U+h^{-1} g_{\mathrm{nl} ; U}\left(h \partial^{+} U\right) \\
& =g^{\prime}(U) \partial^{0} U+\frac{1}{2} h g^{\prime}(U) \partial^{(2)} U+h^{-1} g_{\mathrm{nl} ; U}\left(h \partial^{+} U\right),
\end{aligned}
$$


which yields (F.149). In addition, we compute

$$
\begin{aligned}
g^{+}(U+V)= & h^{-1}\left[g\left(T^{+} U+T^{+} V\right)-g(U+V)\right] \\
= & h^{-1}\left[g\left(T^{+} U+T^{+} V\right)-g\left(U+T^{+} V\right)\right] \\
& +h^{-1}\left[g\left(U+T^{+} V\right)-g(U+V)\right] \\
= & \mathcal{I}_{A}+\mathcal{I}_{B},
\end{aligned}
$$

in which we have

$$
\begin{aligned}
& \mathcal{I}_{A}=h^{-1}\left[g^{\prime}\left(U+T^{+} V\right) h \partial^{+} U+g_{\mathrm{nl} ; U+T^{+} V}\left(h \partial^{+} U\right)\right], \\
& \mathcal{I}_{B}=h^{-1}\left[g^{\prime}(U+V) h \partial^{+} V+g_{\mathrm{nl} ; U+V}\left(h \partial^{+} V\right)\right] .
\end{aligned}
$$

We compute

$$
\begin{aligned}
& \mathcal{I}_{A}=g^{\prime}(U) \partial^{+} U+\left[g^{\prime}\left(U+T^{+} V\right)-g^{\prime}(U)\right] \partial^{+} U+h^{-1} g_{\mathrm{nl} ; U}\left(h \partial^{+} U\right) \\
& +h^{-1}\left[g_{\mathrm{nl} ; U+T^{+} V}\left(h \partial^{+} U\right)-g_{\mathrm{nl} ; U}\left(h \partial^{+} U\right)\right] \\
& =g^{+}(U)+g_{\mathrm{nl} ; U}^{(1)}\left(T^{+} V\right) \partial^{+} U+g^{\prime \prime}(U) \partial^{+} U T^{+} V \\
& +h^{-1}\left[g_{\mathrm{nl} ; U+T^{+} V}\left(h \partial^{+} U\right)-g_{\mathrm{nl} ; U}\left(h \partial^{+} U\right)\right] \text {, }
\end{aligned}
$$

together with

$$
\mathcal{I}_{B}=g^{\prime}(U) \partial^{+} V+\left[g^{\prime}(U+V)-g^{\prime}(U)\right] \partial^{+} V+h^{-1} g_{\mathrm{nl} ; U+V}\left(h \partial^{+} V\right) .
$$

In particular, we see that

$$
\begin{aligned}
g_{\mathrm{nl} ; U}^{+}(V)= & g^{\prime \prime}(U)\left[\partial^{+} U T^{+} V-\left[\partial^{0} U\right] V\right]+g^{\prime}(U)\left[\partial^{+} V-\partial^{0} V\right] \\
& +g_{\mathrm{nl} ; U}^{(1)}\left(T^{+} V\right) \partial^{+} U+h^{-1}\left[g_{\mathrm{nl} ; U+T^{+} V}\left(h \partial^{+} U\right)-g_{\mathrm{nl} ; U}\left(h \partial^{+} U\right)\right] \\
& +\left[g^{\prime}(U+V)-g^{\prime}(U)\right] \partial^{+} V+h^{-1} g_{\mathrm{nl} ; U+V}\left(h \partial^{+} V\right) .
\end{aligned}
$$

Using (F.152) and (F.154), the desired estimate can now be read off from this identity.

Proof of Proposition 7.5 The results follow directly from Lemma F.10.

\section{G Component Estimates II}

In this section we establish the technical estimates for the nonlinearities $\mathcal{Z}^{ \pm}$that were summarized in Proposition 7.4. We treat these computations separately here as they require more delicate techniques than those employed in $\S$ F. Indeed, we need to apply discrete summationby-parts identities to simplify the sums appearing in the definitions (2.16) and (4.4) for $\mathcal{Q}$ and $\mathcal{Z}$.

As a preparation, we recall (3.21) and (4.2), introduce the function

$$
\widetilde{q}(U)=p(U) \mathcal{D}^{\diamond} ;+(U)
$$

and write

$$
\begin{aligned}
& \widetilde{q}_{\text {apx }}(U)=\gamma_{U}^{-2} \partial^{0} U S^{+}\left[\partial^{(2)} U\right], \\
& \widetilde{q}_{\text {lin } ; U}[V]=\gamma_{U}^{-4}\left(2-\gamma_{U}^{2}\right) S^{+}\left[\partial^{(2)} U\right] \partial^{0} V+\gamma_{U}^{-2} \partial^{0} U S^{+}\left[\partial^{(2)} V\right],
\end{aligned}
$$


together with

$$
\widetilde{q}_{\mathrm{nl} ; U}(V)=\widetilde{q}(U+V)-\widetilde{q}(U)-\widetilde{q}_{\operatorname{lin} ; U}[V] .
$$

Lemma G.1 Fix $0<\kappa<\frac{1}{12}$. Then there exists $K>0$ so that the pointwise approximation estimate

$$
\left|\widetilde{q}(U)-\widetilde{q}_{\text {apx }}(U)\right| \leq K h\left[\left|\partial^{+} U\right|+\left|\partial^{(2)} U\right|\right]\left[\left|\partial^{(2)} U\right|+T^{+}\left|\partial^{(2)} U\right|\right]
$$

and the residual bound

$$
\begin{aligned}
&\left|\widetilde{q}_{\mathrm{nl} ; U}(V)\right| \leq K[\left.\left|\partial^{-} V\right|^{2}+\left|\partial^{+} V\right|^{2}+T^{+}\left|\partial^{+} V\right|^{2}\right] \\
&+K\left[\left|\partial^{-} V\right|+\left|\partial^{+} V\right|+T^{+}\left|\partial^{+} V\right|\right]\left[\left|\partial^{(2)} V\right|+T^{+}\left|\partial^{(2)} V\right|\right] \\
&+K h\left[\left|\partial^{-} U\right|+\left|\partial^{+} U\right|+T^{+}\left|\partial^{+} U\right|+\left|\partial^{(2)} U\right|+T^{+}\left|\partial^{(2)} U\right|\right] \\
& \quad \times\left[\left|\partial^{-} V\right|+\left|\partial^{+} V\right|+T^{+}\left|\partial^{+} V\right|+\left|\partial^{(2)} V\right|+T^{+}\left|\partial^{(2)} V\right|\right]
\end{aligned}
$$

both hold for any $h>0$, any $U \in \Omega_{h ; \kappa}$ and any $V \in \ell_{h}^{2}$ for which $U+V \in \Omega_{h ; \kappa}$.

Proof We first note that

$$
\begin{aligned}
p_{\mathrm{apx}}(U) \mathcal{D}_{\mathrm{apx}}^{\diamond_{0} ;+}(U) & =\gamma_{U} \partial^{0} U \gamma_{U}^{-3} S^{+}\left[\partial^{(2)} U\right] \\
& =\gamma_{U}^{-2} \partial^{0} U S^{+}\left[\partial^{(2)} U\right] \\
& =\widetilde{q}_{\text {apx }}(U),
\end{aligned}
$$

while also

$$
\begin{aligned}
p_{\text {apx }}(U) \mathcal{D}_{\text {lin; } ;}^{\diamond ;+}[V]+p_{\text {lin; } U}[V] \mathcal{D}_{\text {apx }}^{\diamond ;+}(U)= & \gamma_{U} \partial^{0} U\left[3 \gamma_{U}^{-5} \partial^{0} U S^{+}\left[\partial^{(2)} U\right] \partial^{0} V+\gamma_{U}^{-3} S^{+}\left[\partial^{(2)} V\right]\right] \\
& +\gamma_{U}^{-1}\left(2 \gamma_{U}^{2}-1\right) \partial^{0} V \gamma_{U}^{-3} S^{+}\left[\partial^{(2)} U\right] \\
= & \left(\frac{2 \gamma_{U}^{2}-1}{\gamma_{U}^{4}}+3 \gamma_{U}^{-4}\left(\partial^{0} U\right)^{2}\right) S^{+}\left[\partial^{(2)} U\right] \partial^{0} V \\
& +\gamma_{U}^{-2} \partial^{0} U S^{+}\left[\partial^{(2)} V\right] \\
= & \widetilde{q}_{\text {lin } ; U}[V] .
\end{aligned}
$$

Lemma C.3 and the definition (4.2) yield the bound

$$
|p(U)|+\left|\mathcal{D}^{\diamond_{0} ;+}(U)\right| \leq C_{1}^{\prime}\left[\left|\partial^{+} U\right|+\left|\partial^{(2)} U\right|+T^{+}\left|\partial^{(2)} U\right|\right] .
$$

Observing that

$$
\begin{aligned}
\left|\widetilde{q}(U)-\widetilde{q}_{\mathrm{apx}}(U)\right| \leq & \left|p(U)-p_{\mathrm{apx}}(U)\right|\left|\mathcal{D}^{\diamond_{0} ;+}(U)\right| \\
& +\left|p_{\mathrm{apx}}(U)\right|\left|\mathcal{D}^{\diamond_{0} ;+}(U)-\mathcal{D}_{\mathrm{apx}}^{\diamond_{0} ;+}(U)\right|,
\end{aligned}
$$

we may hence exploit Lemma's F.5 and F.8 to obtain the first desired estimate.

In addition, the computation

$$
\begin{aligned}
& \widetilde{q}_{\mathrm{nl} ; U}(V)=p(U+V) \mathcal{D}^{\diamond_{0} ;+}(U+V)-p(U) \mathcal{D}^{\diamond_{0} ;+}(U)-\widetilde{q}_{\mathrm{lin} ; U}[V] \\
& =\left[p(U)+p_{\operatorname{lin} ; U}[V]+p_{\mathrm{nl} ; U}(V)\right]\left[\mathcal{D}_{0}^{\diamond_{0} ;+}(U)+\mathcal{D}_{\operatorname{lin} ; U}^{\diamond_{0} ;+}[V]+\mathcal{D}_{\mathrm{nl} ; U}^{\diamond_{0} ;+}(V)\right] \\
& -p(U) \mathcal{D}^{\diamond_{0} ;+}(U)-\widetilde{q}_{\text {lin; } U}[V] \\
& =p_{\text {lin } ; U}[V]\left(\mathcal{D}^{\diamond_{0} ;+}(U)-\mathcal{D}_{\mathrm{apx}}^{\diamond_{0} ;+}(U)\right)+\left(p(U)-p_{\mathrm{apx}}(U)\right) \mathcal{D}_{\operatorname{lin} ; U}^{\diamond_{0} ;+}[V] \\
& +p_{\mathrm{nl} ; U}(V) \mathcal{D}^{\diamond_{0} ;+}(U)+p(U) \mathcal{D}_{\mathrm{nl} ; U}^{\diamond_{0} ;+}(V) \\
& \left(p_{\text {lin; } U}[V]+p_{\mathrm{nl} ; U}(V)\right)\left(\mathcal{D}_{\operatorname{lin} ; U}^{\diamond_{0} ;+}[V]+\mathcal{D}_{\mathrm{nl} ; U}^{\diamond_{0} ;+}(V)\right)
\end{aligned}
$$

together with the bounds in Lemma's F.5 and F.8 yields the second desired estimate. 
Recalling (4.3), we now write

$$
q_{\mathrm{apx}}(U)=\tilde{q}_{\mathrm{apx}}(U), \quad q_{\mathrm{lin} ; U}[V]=\tilde{q}_{\mathrm{lin} ; U}[V],
$$

together with

$$
q_{\mathrm{nl} ; U}(V)=q(U+V)-q(U)-q_{\operatorname{lin} ; U}[V] .
$$

Lemma G.2 Fix $0<\kappa<\frac{1}{12}$. Then there exists $K>0$ so that the pointwise approximation estimate

$$
\left|q(U)-q_{\text {apx }}(U)\right| \leq K h\left[\left|\partial^{+} U\right|+\left|\partial^{(2)} U\right|\right]\left[\left|\partial^{(2)} U\right|+T^{+}\left|\partial^{(2)} U\right|\right]
$$

and the residual bound

$$
\begin{aligned}
\left|q_{\mathrm{nl} ; U}(V)\right| \leq & K\left[\left|\partial^{-} V\right|^{2}+\left|\partial^{+} V\right|^{2}+T^{+}\left|\partial^{+} V\right|^{2}\right] \\
+ & \left.K\left[\left|\partial^{-} V\right|+\left|\partial^{+} V\right|+T^{+}\left|\partial^{+} V\right|\right]\left[\left|\partial^{(2)} V\right|+T^{+}\left|\partial^{(2)} V\right|\right]\right] \\
+ & K h\left[\left|\partial^{-} U\right|+\left|\partial^{+} U\right|+T^{+}\left|\partial^{+} U\right|+\left|\partial^{(2)} U\right|+T^{+}\left|\partial^{(2)} U\right|\right] \\
& \times\left[\left|\partial^{-} V\right|+\left|\partial^{+} V\right|+T^{+}\left|\partial^{+} V\right|+\left|\partial^{(2)} V\right|+T^{+}\left|\partial^{(2)} V\right|\right]
\end{aligned}
$$

both hold for any $h>0$, any $U \in \Omega_{h ; \kappa}$ and any $V \in \ell_{h}^{2}$ for which $U+V \in \Omega_{h ; \kappa}$.

Proof We recall that for every $\tau>0$ there exists $C_{\tau}>0$ so that the inequalities

$$
\mid \begin{aligned}
\ln (1+x)-\ln (1+y)-\frac{1}{1+y}(x-y) \mid & \leq C_{\tau}|x-y|^{2}, \\
{[1+x]^{-1}-1 \mid } & \leq C_{\tau}|x|
\end{aligned}
$$

hold whenever $x+1 \geq \tau$ and $y+1 \geq \tau$.

We now write

$$
\mathcal{I}_{0}=q(U+V)-q(U)-\widetilde{q}(U+V)+\widetilde{q}(U) .
$$

Recalling the definition

$$
q(U)=h^{-1} \ln \left[1+h p(U) \mathcal{D}^{\diamond_{0} ;+}(U)\right]=h^{-1} \ln [1+h \widetilde{q}(U)],
$$

we may compute

$$
\begin{aligned}
\mathcal{I}_{0}= & h^{-1}[\ln [1+h \widetilde{q}(U+V)]-\ln [1+h \widetilde{q}(U)]]-h^{-1}[1+h \widetilde{q}(U)]^{-1}[h \widetilde{q}(U+V)-h \widetilde{q}(U)] \\
& +\left[[1+h \widetilde{q}(U)]^{-1}-1\right][\widetilde{q}(U+V)-\widetilde{q}(U)] .
\end{aligned}
$$

The uniform estimate (4.15) allows us to apply (G.15) with $\tau=\frac{1}{3} \kappa^{2}$ to obtain

$$
\begin{aligned}
\left|\mathcal{I}_{0}\right| \leq & h^{-1} C_{2}^{\prime} h^{2}|\widetilde{q}(U+V)-\widetilde{q}(U)|^{2} \\
& +C_{2}^{\prime} h|\widetilde{q}(U)||\widetilde{q}(U+V)-\widetilde{q}(U)| .
\end{aligned}
$$

Exploiting Lemma G.1 and inspecting (G.1), we see that

$$
\begin{aligned}
\left|\mathcal{I}_{0}\right| \leq & C_{3}^{\prime} h\left[\left|\partial^{-} V\right|^{2}+\left|\partial^{+} V\right|^{2}+T^{+}\left|\partial^{+} V\right|^{2}+\left|\partial^{(2)} V\right|^{2}+T^{+}\left|\partial^{(2)} V\right|^{2}\right] \\
& +C_{3}^{\prime} h\left[\left|\partial^{(2)} U\right|+T^{+}\left|\partial^{(2)} U\right|\right]\left[\left|\partial^{-} V\right|+\left|\partial^{+} V\right|+T^{+}\left|\partial^{+} V\right|+\left|\partial^{(2)} V\right|+T^{+}\left|\partial^{(2)} V\right|\right] .
\end{aligned}
$$

The bound (G.14) now follows from the observation

$$
\begin{aligned}
q_{\mathrm{nl} ; U}(V) & =\mathcal{I}_{0}+\widetilde{q}(U+V)-\widetilde{q}(U)-q_{\operatorname{lin} ; U}[V] \\
& =\mathcal{I}_{0}+\widetilde{q}(U+V)-\widetilde{q}(U)-\widetilde{q}_{\operatorname{lin} ; U}[V] \\
& =\mathcal{I}_{0}+\widetilde{q}_{\mathrm{nl} ; U}(V) .
\end{aligned}
$$


Applying (G.15) with $y=0$ and using

$$
|\widetilde{q}(U)| \leq C_{4}^{\prime} \min \left\{\left|\partial^{+} U\right|,\left|\partial^{(2)} U\right|+T^{+}\left|\partial^{(2)} U\right|\right\},
$$

we find

$$
\begin{aligned}
|q(U)-\widetilde{q}(U)| & \leq h^{-1} C_{5}^{\prime} h^{2}|\widetilde{q}(U)|^{2} \\
& \leq h C_{6}^{\prime}\left|\partial^{+} U\right|\left[\left|\partial^{(2)} U\right|+T^{+}\left|\partial^{(2)} U\right|\right] .
\end{aligned}
$$

The desired bound (G.13) now follows from the identity

$$
q(U)-q_{\mathrm{apx}}(U)=q(U)-\widetilde{q}_{\mathrm{apx}}(U)=q(U)-\widetilde{q}(U)+\widetilde{q}(U)-\widetilde{q}_{\mathrm{apx}}(U) .
$$

We now turn our attention to the function

$$
\mathcal{Q}(U)=\sum_{-; h} q(U)
$$

Recalling the definition (7.28), we write

$$
\begin{aligned}
& \mathcal{Q}_{\text {apx }}(U)=-\ln \gamma_{U}, \\
& \mathcal{Q}_{\text {lin } ; U}[V]=\gamma_{U}^{-2}\left[\partial^{0} U\right] \partial^{0} V+\sum_{-; h} \mathcal{E}_{\text {sm }}(U) \partial^{0} V,
\end{aligned}
$$

together with

$$
\mathcal{Q}_{\mathrm{nl} ; U}(V)=\mathcal{Q}(U+V)-\mathcal{Q}(U)-\mathcal{Q}_{\operatorname{lin} ; U}[V] .
$$

Lemma G.3 Fix $0<\kappa<\frac{1}{12}$. Then there exists $K>0$ so that the pointwise approximation estimate

$$
\left|\mathcal{Q}(U)-\mathcal{Q}_{\text {apx }}(U)\right| \leq K h
$$

holds for all $h>0$ and all $U \in \Omega_{h ; \kappa}$.

Proof Writing

$$
\begin{aligned}
\mathcal{Q}_{\mathrm{apx} ; I}(U) & =\sum_{-; h} q_{\mathrm{apx}}(U) \\
& =\sum_{-; h}^{-2} \gamma_{U}^{-2} \partial^{0} U S^{+}\left[\partial^{(2)} U\right],
\end{aligned}
$$

Lemma G.2 implies that

$$
\begin{aligned}
\left|\mathcal{Q}(U)-\mathcal{Q}_{\text {apx } ; I}(U)\right| & \leq \sum_{-; h}\left|q(U)-q_{\text {apx }}(U)\right| \\
& \leq C_{1}^{\prime} h\left[\left\|\partial^{+} U\right\|_{\ell_{h}^{2}}+\left\|\partial^{+} \partial^{+} U\right\|_{\ell_{h}^{2}}\right]\left\|\partial^{+} \partial^{+} U\right\|_{\ell_{h}^{2}} \\
& \leq C_{2}^{\prime} h .
\end{aligned}
$$

On the other hand, Lemma D.9 yields the bound

$$
\left|\mathcal{Q}_{\mathrm{apx} ; I}(U)+\ln \left[\gamma_{U}\right]\right|=\left|\mathcal{Q}_{\mathrm{apx} ; I}(U)-\mathcal{Q}_{\mathrm{apx}}(U)\right| \leq C_{3}^{\prime} h,
$$

which completes the proof.

Lemma G.4 Fix $0<\kappa<\frac{1}{12}$. Then there exists $K>0$ so that the the residual bound

$$
\left|\mathcal{Q}_{\mathrm{nl} ; U}(V)\right| \leq K\left[\left\|\partial^{+} V\right\|_{\ell_{h}^{2}}+h\right]\left[\left\|\partial^{+} V\right\|_{\ell_{h}^{2}}+\left\|\partial^{+} \partial^{+} V\right\|_{\ell_{h}^{2}}\right]+K h\left|\partial^{0} V\right|
$$

holds for any $h>0$, any $U \in \Omega_{h ; \kappa}$ and any $V \in \ell_{h}^{2}$ for which $U+V \in \Omega_{h ; \kappa}$. 
Proof Writing

$$
\begin{aligned}
\mathcal{Q}_{\text {lin } ; U ; I}[V] & =\sum_{-; h} q_{\operatorname{lin} ; U}[V] \\
& =\sum_{-; h}\left[\gamma_{U}^{-4}\left(2-\gamma_{U}^{2}\right) S^{+}\left[\partial^{(2)} U\right] \partial^{0} V+\gamma_{U}^{-2} \partial^{0} U S^{+}\left[\partial^{(2)} V\right]\right],
\end{aligned}
$$

we compute

$$
\begin{aligned}
\left|\mathcal{Q}(U+V)-\mathcal{Q}(U)-\mathcal{Q}_{\operatorname{lin} ; U ; I}[V]\right| & \leq \sum_{-; h}\left|q_{\mathrm{nl} ; U}(V)\right| \\
& \leq K\left[\left\|\partial^{+} V\right\|_{\ell_{h}^{2}}+h\right]\left[\left\|\partial^{+} V\right\|_{\ell_{h}^{2}}+\left\|\partial^{+} \partial^{+} V\right\|_{\ell_{h}^{2}}\right] .
\end{aligned}
$$

Recalling the definition (7.28), we see that

$$
\begin{aligned}
\mathcal{Q}_{\text {lin } ; U ; I}[V]= & \sum_{-; h}\left[T^{-}\left[\gamma_{U}^{-4}\left(2-\gamma_{U}^{2}\right) S^{+}\left[\partial^{(2)} U\right]\right] \partial^{0} V+\gamma_{U}^{-2} \partial^{0} U S^{+}\left[\partial^{(2)} V\right]\right] \\
& +\sum_{-; h} \mathcal{E}_{\text {sm }}(U) \partial^{0} V .
\end{aligned}
$$

The summation-by-parts identity (3.13) implies that

$$
\begin{aligned}
\sum_{-; h} \gamma_{U}^{-2} \partial^{0} U S^{+}\left[\partial^{(2)} V\right]= & \sum_{-; h} \gamma_{U}^{-2}\left[\partial^{0} U\right] \partial^{+} \partial^{0} V \\
= & T^{-}\left[\gamma_{U}^{-2} \partial^{0} U\right] \partial^{0} V-\sum_{-; h} \partial^{-}\left[\gamma_{U}^{-2} \partial^{0} U\right] \partial^{0} V \\
= & \gamma_{U}^{-2}\left[\partial^{0} U\right] \partial^{0} V-h \partial^{-}\left[\gamma_{U}^{-2} \partial^{0} U\right] \partial^{0} V \\
& -\sum_{-; h} T^{-}\left[\partial^{+}\left[\gamma_{U}^{-2} \partial^{0} U\right]\right] \partial^{0} V .
\end{aligned}
$$

In particular, upon writing

$$
\begin{aligned}
\mathcal{Q}_{\operatorname{lin} ; U ; I I}[V]= & \sum_{-; h} T^{-}\left[\gamma_{U}^{-4}\left(2-\gamma_{U}^{2}\right) S^{+}\left[\partial^{(2)} U\right]-\partial^{+}\left[\gamma_{U}^{-2} \partial^{0} U\right]\right] \partial^{0} V \\
& +\gamma_{U}^{-2} \partial^{0} U \partial^{0} V+\sum_{-; h} \mathcal{E}_{\mathrm{sm}}(U) \partial^{0} V
\end{aligned}
$$

we see that

$$
\left|Q_{\text {lin } ; U ; I I}[V]-\mathcal{Q}_{\operatorname{lin} ; U ; I}[V]\right|=h\left|\partial^{-}\left[\gamma_{U}^{-2} \partial^{0} U\right] \partial^{0} V\right| \leq h C_{1}^{\prime}\left|\partial^{0} V\right| .
$$

Observing that

$$
Q_{\operatorname{lin} ; U ; I I}[V]-\mathcal{Q}_{\operatorname{lin} ; U}[V]=\sum_{-; h} T^{-}\left[\gamma_{U}^{-4}\left(2-\gamma_{U}^{2}\right) S^{+}\left[\partial^{(2)} U\right]-\partial^{+}\left[\gamma_{U}^{-2} \partial^{0} U\right]\right] \partial^{0} V,
$$

we may apply Lemma D.8 to conclude

$$
\left|Q_{\operatorname{lin} ; U ; I I}[V]-\mathcal{Q}_{\operatorname{lin} ; U}[V]\right| \leq h C_{2}^{\prime}\left\|\partial^{+} V\right\|_{\ell_{h}^{2}}
$$

as desired.

We now recall (4.4) together with the definitions

$$
\begin{array}{ll}
\mathcal{Z}_{\text {apx }}^{+}(U)=\gamma_{U}^{-1}, & \mathcal{Z}_{\text {lin } ; U}^{+}[V]=\gamma_{U}^{-3}\left[\partial^{0} U\right] \partial^{0} V+\gamma_{U}^{-1} \sum_{-; h} \mathcal{E}_{\text {sm }}(U) \partial^{0} V, \\
\mathcal{Z}_{\text {apx }}^{-}(U)=\gamma_{U}, & \mathcal{Z}_{\text {lin } ; U}^{-}[V]=-\gamma_{U}^{-1}\left[\partial^{0} U\right] \partial^{0} V-\gamma_{U} \sum_{-; h} \mathcal{E}_{\text {sm }}(U) \partial^{0} V
\end{array}
$$

and write

$$
\mathcal{Z}_{\mathrm{nl} ; U}^{ \pm}(V)=\mathcal{Z}^{ \pm}(U+V)-\mathcal{Z}^{ \pm}(U)-\mathcal{Z}_{\operatorname{lin} ; U}^{ \pm}[V]
$$


Lemma G.5 Fix $0<\kappa<\frac{1}{12}$. Then there exists $K>0$ so that the pointwise approximation estimate

$$
\left|\mathcal{Z}^{+}(U)-\mathcal{Z}_{\text {apx }}^{+}(U)\right|+\left|\mathcal{Z}^{-}(U)-\mathcal{Z}_{\text {apx }}^{-}(U)\right| \leq K h
$$

and the residual bound

$$
\begin{aligned}
\left|\mathcal{Z}_{\mathrm{nl}}^{+}(V)\right|+\left|\mathcal{Z}_{\mathrm{nl}}^{-}(V)\right| \leq K[ & \left.\left\|\partial^{+} V\right\|_{\ell_{h}^{2}}^{2}+\left\|\partial^{+} \partial^{+} V\right\|_{\ell_{h}^{2}}^{2}\right] \\
& +K h\left[\left\|\partial^{+} V\right\|_{\ell_{h}^{2}}+\left\|\partial^{+} \partial^{+} V\right\|_{\ell_{h}^{2}}\right]+K h\left|\partial^{0} V\right|
\end{aligned}
$$

both hold for any $h>0$, any $U \in \Omega_{h ; \kappa}$ and any $V \in \ell_{h}^{2}$ for which $U+V \in \Omega_{h ; \kappa}$.

Proof Motivated by the definitions (4.4), we write

$$
f^{+}(x)=\exp [x], \quad f^{-}(x)=\exp [-x]
$$

together with

$$
\phi\left(\partial^{+} U, \partial^{(2)} U\right)=\mathcal{Q}(U)
$$

and finally

$$
P^{ \pm}\left(\partial^{+} U, \partial^{(2)} U\right)=f^{ \pm}\left(\phi\left(\partial^{+} U, \partial^{(2)} U\right)\right) .
$$

This allows one to verify that

$$
\mathcal{Z}^{ \pm}(U)=P^{ \pm}\left(\partial^{+} U, \partial^{(2)} U\right) .
$$

Recalling (7.28), we now define the approximants

$$
\begin{aligned}
& \phi_{\text {apx }}\left(\partial^{+} U, \partial^{(2)} U\right)=-\ln \gamma_{U}, \\
& \phi_{\text {lin } ; U}\left[\partial^{+} V, \partial^{(2)} V\right]=\gamma_{U}^{-2}\left[\partial^{0} U\right] \partial^{0} V+\sum_{-; h} \mathcal{E}_{\text {sm }}(U) \partial^{0} V .
\end{aligned}
$$

Lemma G.4 provides the pointwise bound

$$
\left|\phi\left(\partial^{+} U, \partial^{(2)} U\right)-\phi_{\text {apx }}\left(\partial^{+} U, \partial^{(2)} U\right)\right|=\left|\mathcal{Q}(U)-\mathcal{Q}_{\text {apx }}(U)\right| \leq C_{1}^{\prime} h,
$$

together with

$$
\begin{aligned}
\left|\phi_{\mathrm{nl} ; U}[V]\right| & =\left|\mathcal{Q}_{\mathrm{nl} ; U}(V)\right| \\
& \leq C_{1}^{\prime}\left[\left\|\partial^{+} V\right\|_{\ell_{h}^{2}}+h\right]\left[\left\|\partial^{+} V\right\|_{\ell_{h}^{2}}+\left\|\partial^{+} \partial^{+} V\right\|_{\ell_{h}^{2}}\right]+C_{1}^{\prime} h\left|\partial^{0} V\right| .
\end{aligned}
$$

Noting that $D f^{ \pm}(x)= \pm f^{ \pm}(x)$, we see that the functions defined in (E.7) satisfy

$$
\begin{aligned}
P_{\mathrm{apx}}^{ \pm}(U) & =f^{ \pm}\left(\phi_{\mathrm{apx}}\left(\partial^{+} U, \partial^{(2)} U\right)\right) \\
& =\exp \left[\mp \ln \left[\gamma_{U}\right]\right] \\
& =\gamma_{U}^{\mp 1},
\end{aligned}
$$

together with

$$
\begin{aligned}
P_{\operatorname{lin} ; U}^{ \pm}[V] & = \pm f^{ \pm}\left(-\ln \left[\gamma_{U}\right]\right)\left[\phi_{\operatorname{lin} ; U}[V]\right] \\
& = \pm \gamma_{U}^{\mp 1}\left[\gamma_{U}^{-2}\left[\partial^{0} U\right] \partial^{0} V+\sum_{-; h} \mathcal{E}_{\mathrm{sm}}(U) \partial^{0} V\right] \\
& = \pm \gamma_{U}^{-(2 \pm 1)}\left[\partial^{0} U\right] \partial^{0} V \pm \gamma_{U}^{\mp 1} \sum_{-; h} \mathcal{E}_{\mathrm{sm}}(U) \partial^{0} V
\end{aligned}
$$

The desired estimates now follow from Lemma E.1.

Proof of Proposition 7.4 The results follow directly from Lemma G.5. 


\section{H List of Symbols}

The tables below summarize the most important symbols that are introduced at various locations in the main text. We have chosen to only include symbols that occur in multiple sections.

\section{Discrete calculus}

\begin{tabular}{lll}
\hline$\partial^{ \pm}, \partial^{0}$ & $(2.12)$ & First differences, scaled with $h$ \\
$\partial^{(2)}=\partial^{+} \partial^{-}$ & $(3.3)$ & Second difference, scaled with $h^{2}$ \\
$T^{ \pm}$ & $(3.1)$ & Translation of sequence by $\pm h$ \\
$S^{ \pm}, P^{ \pm}$ & $(3.2)$ & Sum and product with translated sequence \\
$\sum_{-; h}, \sum_{+; h}$, & $(3.9)$ & Left and right discrete integral \\
\hline
\end{tabular}

\section{Sequence spaces}

\begin{tabular}{lll}
\hline$\ell_{h}^{2 ; 1}, \ell_{h}^{2 ; 2}, \ell_{h}^{2 ; 3}$ & $(3.30)$ and $(3.34)$ & $\ell^{2}(h \mathbb{Z} ; \mathbb{R})$ with scaled inner products \\
$\ell_{h}^{\infty}, \ell_{h}^{\infty} ; 1, \ell_{h}^{\infty ; 2}$ & $(3.32)$ and (3.35) & $\ell^{\infty}(h \mathbb{Z} ; \mathbb{R})$ with scaled derivatives \\
$U_{\text {ref } ; *}, U_{\text {ref } ; \kappa}$ & $(3.36)-(3.38)$ & Reference functions connecting $0 \mapsto 1$ \\
$\mathcal{V}_{h ; \kappa}$ & $(3.39)$ & Admissible perturbations from $U_{\text {ref } ; \kappa}$ \\
$\Omega_{h ; \kappa}$ & $(3.40)$ & State space for $U$ \\
\hline
\end{tabular}

\section{Main nonlinearities}

\begin{tabular}{lll}
$\mathcal{G}, \mathcal{G}^{+}$ & $(6.1)$ and $(6.2)$ & Right-hand-side of main reduced system $(2.25)$ \\
$\mathcal{Y}$ & $(2.17)$ and (4.8) & Governs gridpoint speed, ie $\dot{x}=\mathcal{Y}$ \\
$\mathcal{Q}$ & $(2.16)$ and $(4.7)$ & Integrating factor for $\mathcal{Y}$ \\
$\mathcal{Z}^{ \pm}$ & $(4.4)$ & Exponential of $\pm \mathcal{Q}$ \\
\hline
\end{tabular}

\section{Adaptive discrete derivatives}

\begin{tabular}{lll}
\hline$r_{U}^{0}, \gamma_{U}$ & $(3.16)$ and $(7.6)$ & Gridpoint spacing \\
$\mathcal{D}^{\diamond_{ \pm}, \mathcal{D}^{\diamond_{0}}}$ & $(3.17)$ & Adaptive first discrete derivatives \\
$\mathcal{D}^{\diamond_{0} ;+}, \mathcal{D}^{\diamond_{-} ;+}, \mathcal{D}^{\diamond_{+} ;+}$ & $(3.21)$ & Mixed second discrete derivatives \\
$\mathcal{I}_{0}^{\diamond_{-} ;+}, \mathcal{I}_{0}^{\diamond_{0} ;+}, \mathcal{I}_{s}^{\diamond_{0} ;+}$ & $(3.22)$ & Supporting functions for $\mathcal{D}^{\diamond_{-} ;+}$ \\
$\mathcal{D}^{\diamond_{0}}$ & $(3.26)$ & Adaptive second discrete derivative \\
$\mathcal{D}^{\diamond_{0} ;+}$ & $(3.27)$ & Mixed third discrete derivative \\
$\mathcal{I}_{0 s}^{\diamond \diamond_{0} ;+}, \mathcal{I}_{s s}^{\diamond \diamond_{0} ;+}, \mathcal{I}_{+}^{\diamond \diamond_{0} ;+}$ & $(3.28)$ & Supporting functions for $\mathcal{D}^{\diamond_{0} ;+}$ \\
\hline
\end{tabular}




\section{Auxiliary functions}

\begin{tabular}{lll}
$\widetilde{p}, p, q$ & $(4.2)$ and (4.3) & Supporting functions for $\mathcal{Y}$ \\
$p_{A}^{\diamond+}, p_{B}^{\diamond+}, p^{\diamond 0}$ & (6.3) & Used to compute $\partial^{+} p$ \\
\hline
\end{tabular}

\section{Decomposition of $\mathcal{G}$ and $\mathcal{G}^{+}$}

\begin{tabular}{lll}
\hline $\mathcal{G}_{A}, \mathcal{G}_{B}, \mathcal{G}_{C}, \mathcal{G}_{D}$ & $(6.9)$ & Four components of $\mathcal{G}$ \\
$\mathcal{G}_{A^{\prime} a}^{+}$ & $(6.14)$ & Component of $\mathcal{G}^{+}$that contains third derivative \\
$\mathcal{G}_{A^{\prime} b}^{+}, \mathcal{G}_{A^{\prime} c^{+}}^{+}, \mathcal{G}_{B^{\prime}}, \mathcal{G}_{C^{\prime}}, \mathcal{G}_{D^{\prime}}$ & $(6.14)$ and (6.15) & Remaining 5 components of $\mathcal{G}^{+}$ \\
$\mathcal{Y}_{1}, \mathcal{Y}_{1}^{+}, \mathcal{Y}_{2 b}^{+}$ & $(6.7)$ and $(6.11)$ & Supporting functions for $\mathcal{G}_{\#}$ and $\mathcal{G}_{\#}^{+}$ \\
$\mathcal{Y}_{2}, \mathcal{Y}_{2}^{+}, \mathcal{Y}_{2 a}^{+}$ & $(6.7),(6.11),(6.13)$ & Contain highest derivatives in $\mathcal{G}_{\#}$ and $\mathcal{G}_{\#}^{+}$ \\
$\mathcal{X}_{A}, \mathcal{X}_{B}, \mathcal{X}_{C}, \mathcal{X}_{D}$ & $(6.8)$ & Appear only in corresponding $\mathcal{G}_{\#}$ and $\mathcal{G}_{\#}^{+}$ \\
\hline
\end{tabular}

\section{Approximation framework}

\begin{tabular}{lll}
\hline$S_{\text {full }}, \bar{S}_{\text {full }}, S_{2 ; \text { fix }}, \bar{S}_{2 ; \text { fix }}$ & $(7.1)$ & Bounds for the seminorms in condition $(h f)$ \\
$T_{\text {safe }}, \bar{T}_{\text {safe }}, T_{\infty ; \text { opt }}, \bar{T}_{\infty ; \text { opt }}$ & $(7.2)$ & Bounds for the linear terms in condition $(h f)_{\text {lin }}$ \\
$\mathcal{E}_{\mathrm{nl}}, \overline{\mathcal{E}}_{\mathrm{nl}}$ & $(7.3)$ & Bounds for the nonlinear terms in condition $(h f)_{\mathrm{nl}}$ \\
$Q_{f}$ for $f \in \overline{\mathcal{S}}_{\mathrm{nl}}$ & $\S 7.1$ & Exponents for which condition $(h f)$ holds \\
$Q_{f ; \text { pref }}$ for $f \in \mathcal{S}_{\mathrm{nl}}$ & $(6.18)$ & Preferred exponents for $\mathcal{G}$ bounds; see $\S 7.2$ \\
$\bar{Q}_{f ; \text { pref }}$ for $f \in \overline{\mathcal{S}}_{\mathrm{nl}}$ & $(6.20)$ & Preferred exponents for $\mathcal{G}^{+}$bounds; see $\S 7.2$ \\
$Q_{f ; \operatorname{lin}}^{A}, Q_{f ; \operatorname{lin}}^{B}$ for $f \in \overline{\mathcal{S}}_{\mathrm{nl}}$ & $\S 7.1$ & Exponents for which condition $(h f)_{\text {lin holds }}$ \\
$Q_{f ; \mathrm{nl}}^{A}, Q_{f ; \mathrm{nl}}^{B}$ for $f \in \overline{\mathcal{S}}_{\mathrm{nl}}$ & $\S 7.1$ & Exponents for which condition $(h f)_{\mathrm{nl}}$ holds \\
\hline
\end{tabular}

\section{Approximants}

\begin{tabular}{lll}
\hline$f_{\text {apx }}$ for $f \in \overline{\mathcal{S}}_{\text {nl }}$ & $\S 7.1$ & Simplification for $f ;$ see condition $(h f)$ \\
$f_{\text {lin } ; U}$ for $f \in \overline{\mathcal{S}}_{\mathrm{nl}}$ & $\S 7.1$ & Simplification for $D f(U)$; see condition $(h f)$ \\
\hline
\end{tabular}

\section{References}

1. Aronson, D.G., Weinberger, H.F.: Multidimensional nonlinear diffusion arising in population genetics. Adv. Math. 30(1), 33-76 (1978)

2. Babuška, I., Rheinboldt, W.C.: Analysis of optimal finite-element meshes in $R^{1}$. Math. Comput. 33(146), 435-463 (1979)

3. Bates, P.W., Chmaj, A.: A discrete convolution model for phase transitions. Arch. Ration. Mech. Anal. 150, 281-305 (1999)

4. Beckett, G., Mackenzie, J.A.: On a uniformly accurate finite difference approximation of a singularly perturbed reaction-diffusion problem using grid equidistribution. J. Comput. Appl. Math. 131(1-2), 381-405 (2001)

5. Bell, J., Cosner, C.: Threshold behavior and propagation for nonlinear differential-difference systems motivated by modeling myelinated axons. Q. Appl. Math. 42(1), 1-14 (1984)

6. Berestycki, H., Hamel, F., Matano, H.: Bistable traveling waves around an obstacle. Commun. Pure Appl. Math. 62(6), 729-788 (2009) 
7. Brucal-Hallare, M., Van Vleck, E.S.: Traveling wavefronts in an antidiffusion lattice Nagumo model. SIAM J. Appl. Dyn. Syst. 10, 921-959 (2011)

8. Budd, C.J., Huang, W., Russell, R.D.: Moving mesh methods for problems with blow-up. SIAM J. Sci. Comput. 17(2), 305-327 (1996)

9. Budd, C.J., Huang, W., Russell, R.D.: Adaptivity with moving grids. Acta Numer. 18, 111-241 (2009)

10. Cahn, J.W.: Theory of crystal growth and interface motion in crystalline materials. Acta Met. 8, 554-562 (1960)

11. Carpenter, G.: A geometric approach to singular perturbation problems with applications to nerve impulse equations. J. Differ. Equ. 23, 335-367 (1977)

12. Carter, P., de Rijk, B., Sandstede, B.: Stability of traveling pulses with oscillatory tails in the FitzHughNagumo system. J. Nonlinear Sci. 26(5), 1369-1444 (2016)

13. Carter, P., Sandstede, B.: Fast pulses with oscillatory tails in the Fitzhugh-Nagumo system. SIAM J. Math. Anal. 47(5), 3393-3441 (2015)

14. Chen, X., Guo, J.S., Wu, C.C.: Traveling waves in discrete periodic media for bistable dynamics. Arch. Ration. Mech. Anal. 189, 189-236 (2008)

15. Chow, S.N., Mallet-Paret, J., Shen, W.: Traveling waves in lattice dynamical systems. J. Differ. Equ. 149, 248-291 (1998)

16. de Boor, C.: Good approximation by splines with variable knots. II. In: Conference on the Numerical Solution of Differential Equations (Univ. Dundee, Dundee, 1973). Springer, Berlin, pp. 12-20. Lecture Notes in Math., Vol. 363 (1974)

17. de Camino-Beck, T., Lewis, M.: Invasion with stage-structured coupled map lattices: application to the spread of scentless chamomile. Ecol. Model. 220(23), 3394-3403 (2009)

18. Elmer, C.E., Van Vleck, E.S.: Anisotropy, propagation failure, and wave speedup in traveling waves of discretizations of a Nagumo PDE. J. Comput. Phys. 185(2), 562-582 (2003)

19. Elmer, C.E., Van Vleck, E.S.: Existence of monotone traveling fronts for BDF discretizations of bistable reaction-diffusion equations. Dyn. Contin. Discrete Impuls. Syst. Ser. A Math. Anal. 10(1-3), 389-402. Second International Conference on Dynamics of Continuous, Discrete and Impulsive Systems (London, ON, 2001) (2003)

20. Elmer, C.E., Van Vleck, E.S.: Dynamics of monotone travelling fronts for discretizations of Nagumo PDEs. Nonlinearity 18, 1605-1628 (2005)

21. Fife, P.C., McLeod, J.B.: The approach of solutions of nonlinear diffusion equations to travelling front solutions. Arch. Ration. Mech. Anal. 65(4), 335-361 (1977)

22. FitzHugh, R.: Mathematical models of excitation and propagation in nerve. Publisher Unknown (1966)

23. Fitzhugh, R.: Motion picture of nerve impulse propagation using computer animation. J. Appl. Physiol. 25(5), 628-630 (1968)

24. Hastings, S.: On travelling wave solutions of the Hodgkin-Huxley equations. Arch. Ration. Mech. Anal. 60, 229-257 (1976)

25. Hawken, D.F., Gottlieb, J.J., Hansen, J.S.: Review of some adaptive node-movement techniques in finiteelement and finite-difference solutions of partial differential equations. J. Comput. Phys. 95(2), 254-302 (1991)

26. Huang, W., Kamenski, L.: On the mesh nonsingularity of the moving mesh PDE method. Math. Comput. 87(312), 1887-1911 (2018)

27. Huang, W., Ren, Y., Russell, R.D.: Moving mesh methods based on moving mesh partial differential equations. J. Comput. Phys. 113(2), 279-290 (1994)

28. Huang, W., Ren, Y., Russell, R.D.: Moving mesh partial differential equations (MMPDES) based on the equidistribution principle. SIAM J. Numer. Anal. 31(3), 709-730 (1994)

29. uang, W., Russell, R.D.: Adaptive Mesh Movement in 1D. In: Adaptive Moving Mesh Methods. Springer, pp. 27-135 (2011)

30. Hupkes, H.J., Morelli, L., Schouten-Straatman, W.M., Van Vleck, E.S.: Traveling Waves and Pattern Formation for Spatially Discrete Bistable Reaction-Diffusion Equations. International Conference on Difference Equations and Applications, 55-112 (2018)

31. Hupkes, H.J., Van Vleck, E.S.: Travelling waves for adaptive grid discretizations of reaction diffusion systems II: Linear theory. J. Dyn. Differ. Equ. 1-50 (2021)

32. Hupkes, H.J., Van Vleck, E.S.: Travelling waves for adaptive grid discretizations of reaction diffusion systems III: Nonlinear theory. Preprint (2019)

33. Hupkes, H.J., Van Vleck, E.S.: Travelling waves for complete discretizations of reaction diffusion systems. J. Dyn. Differ. Equ. 28(3), 955-1006 (2016)

34. Jones, C.K.R.T.: Stability of the travelling wave solutions of the FitzHugh-Nagumo system. Trans. AMS 286, 431-469 (1984) 
35. Keener, J.P.: Propagation and its failure in coupled systems of discrete excitable cells. SIAM J. Appl. Math. 47, 556-572 (1987)

36. Kopteva, N., Stynes, M.: A robust adaptive method for a quasi-linear one-dimensional convectiondiffusion problem. SIAM J. Numer. Anal. 39(4), 1446-1467 (2001)

37. Krupa, M., Sandstede, B., Szmolyan, P.: Fast and slow waves in the FitzHugh-Nagumo equation. J. Diff. Equ. 133, 49-97 (1997)

38. Mallet-Paret, J.: The global structure of traveling waves in spatially discrete dynamical systems. J. Dyn. Differ. Equ. 11, 49-128 (1999)

39. Matano, H., Nara, M.: Large time behavior of disturbed planar fronts in the Allen-Cahn equation. J. Differ. Equ. 251(12), 3522-3557 (2011)

40. Qiu, Y., Sloan, D.M.: Numerical solution of Fisher's equation using a moving mesh method. J. Comput. Phys. 146(2), 726-746 (1998)

41. Qiu, Y., Sloan, D.M.: Analysis of difference approximations to a singularly perturbed two-point boundary value problem on an adaptively generated grid. J. Comput. Appl. Math. 101(1-2), 1-25 (1999)

42. Qiu, Y., Sloan, D.M., Tang, T.: Numerical solution of a singularly perturbed two-point boundary value problem using equidistribution: analysis of convergence. J. Comput. Appl. Math. 116(1), 121-143 (2000)

43. Ren, Y., Russell, R.D.: Moving mesh techniques based upon equidistribution, and their stability. SIAM J. Sci. Stat. Comput. 13(6), 1265-1286 (1992)

44. Roussier, V.: Stability of radially symmetric travelling waves in reaction-diffusion equations. In: Annales de l'Institut Henri Poincare (C) Non Linear Analysis, Vol. 21. Elsevier, pp. 341-379 (2004)

45. Sattinger, D.: Weighted norms for the stability of traveling waves. J. Differ. Equ. 25(1), 130-144 (1977)

46. Schouten-Straatman, W.M., Hupkes, H.J.: Nonlinear stability of pulse solutions for the discrete FitzHughNagumo equation with infinite-range interactions. Discrete Contin. Dyn. Syst. A 39(9), 5017-5083 (2019)

47. Stehlık, P., Volek, J.: Maximum Principles for Discrete and Semidiscrete Reaction-Diffusion Equation. Discrete Dynamics in Nature and Society (2015)

48. Turing, A.M.: The chemical basis of morphogenesis. Philos. Trans. R. Soc. Lond. B Biol. Sci. 237(641), 37-72 (1952)

49. Vainchtein, A., Van Vleck, E.S.: Nucleation and propagation of phase mixtures in a bistable chain. Phys. Rev. B 79, 144123 (2009)

50. Wang, Y., Liao, X., Xiao, D., Wong, K.-W.: One-way Hash function construction based on 2D coupled map lattices. Inf. Sci. 178(5), 1391-1406 (2008)

51. Weinberger, H.F.: Genetic wave propagation, convex sets, and semi-infinite programming. In: Constructive approaches to mathematical models (Proc. Conf. in honor of R. J. Duffin, Pittsburgh, Pa., 1978). New York: Academic Press, pp. 293-317 (1979)

52. Weinberger, H.F.: Long-time behavior of a class of biological models. SIAM J. Math. Anal. 13(3), 353396 (1982)

53. Zinner, B.: Stability of traveling wavefronts for the discrete Nagumo equation. J. Diff. Equ. 96, 1-27 (1992)

Publisher's Note Springer Nature remains neutral with regard to jurisdictional claims in published maps and institutional affiliations. 